\title{
Effector biology of the sugar beet pathogen Cercospora beticola
}

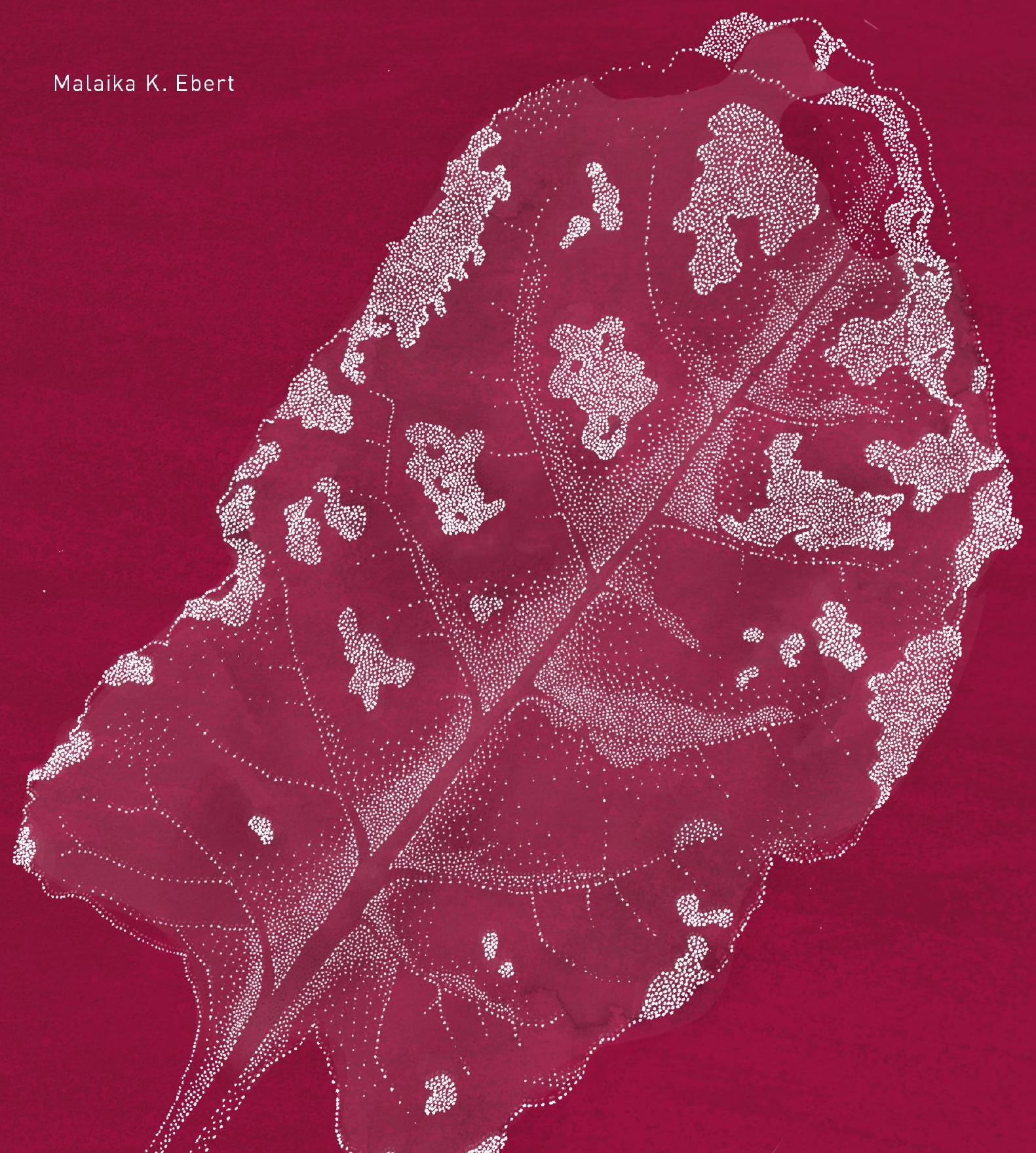




\section{Propositions}

1. CbNip1 evolved to compensate for the lack of cercosporin functionality in the dark

(this thesis)

2. Microbial toxin auto-resistance mechanisms have the potential to provide durable resistance in crop plants.

(this thesis)

3. After high-throughput genotyping, high-throughput phenotyping is the next major challenge plant science researchers have to overcome.

4. Integration of the knowledge on crop-associated microbiomes into farming techniques has the potential to improve farming.

5. Every job application deserves a reply.

6. Drones will revolutionize modern farming.

Propositions belonging to the thesis, entitled

'Effector biology of the sugar beet pathogen Cercospora beticola'.

Malaika Karolina Ebert

Wageningen, 06 September 2018. 
Effector biology of the sugar beet pathogen Cercospora beticola 


\section{Thesis committee}

\section{Promotor}

Prof Dr B.P.H.J. Thomma

Professor of Phytopathology

Wageningen University \& Research

\section{Co-promotor}

DrM.D. Bolton

Research Plant Pathologist

United States Department of Agriculture - ARS Fargo, USA

Adjunct professor Department of Plant Pathology

North Dakota State University, Fargo, USA

\section{Other members}

Prof Dr R. Hall, Wageningen University \& Research

Prof Dr G. de Deyn, Wageningen University \& Research

Dr E. Limpens, Wageningen University \& Research

Dr J. Collemare, Westerdijk Fungal Biodiversity Institute, Utrecht

This thesis was conducted under the auspices of the Graduate School of Experimental Plant Sciences. 


\title{
Effector biology of the sugar beet pathogen Cercospora beticola
}

\author{
Malaika K. Ebert
}

\section{Thesis}

submitted in fulfilment of the requirements for the degree of doctor at Wageningen University

by the authority of the Rector Magnificus,

Prof. Dr A.P.J. Mol,

in the presence of the

Thesis Committee appointed by the Academic Board

to be defended in public

on Thursday 6 September 2018

at 11 a.m. in the Aula. 
Malaika K. Ebert

Effector biology of the sugar beet pathogen Cercospora beticola, 160 pages.

PhD thesis, Wageningen University, Wageningen, The Netherlands (2018) With references, with summary in English

ISBN: 978-94-6343-302-0

DOI: $10.18174 / 453825$ 
Index

Chapter 1

General introduction and outline of the thesis

\section{Chapter 2}

Tools of the crook - infection strategies of fungal plant pathogens

\section{Chapter 3}

Homologs of Verticillium dahliae effector Ave1 contribute to virulence of fungal pathogens of diverse plant hosts

\section{Chapter 4}

Identification of Cercospora beticola necrosis-inducing effector CbNip1

\section{Chapter 5}

Gene cluster conservation provides insight into

cercosporin biosynthesis and extends production to the genus Colletotrichum

\section{Chapter 6}

Gene cluster conservation identifies melanin and perylenequinone biosynthesis pathways in multiple plant pathogenic fungi

\section{Chapter 7}

General discussion

Acknowledgements

About the author 

General introduction and outline of the thesis 


\section{Introduction}

Interactions between plant pathogenic fungi and their hosts are comprised of various, complex events that often occur simultaneously. In order to successfully establish disease, the fungus has to circumvent the plant innate immune system that relies on immune receptors to sense invading pathogens. The initial model to elucidate interactions between pathogen-derived ligands and plant immune receptors was formulated by Flor (1942) (1), and is better known as the "gene-for-gene hypothesis". It proposes that race-specific resistance in plants is determined by corresponding gene pairs, more precisely the products of resistance genes ( $R$ genes) in the plant are able to recognize the products of corresponding pathogen-derived avirulence genes (Avrs). A couple of years later, a new model was introduced, the so called "zigzag model". This model illustrates that general, non-race specific, elicitors also known as microbe-associated molecular patterns (MAMPs) can be perceived by the plant through pattern recognition receptors (PRRs) located on the cell surface which will elicit a first immune defense response named MAMP-triggered immunity (MTI) (2-7). In turn, pathogens have developed effectors to counteract MTI which leads to effector-triggered susceptibility (ETS). Detection of effectors by intracellular receptors encoded by $R$ genes results in effector-triggered immunity (ETI) unless the pathogen is able to suppress (ETI) by loss or modification of the recognized effectors, or utilization of novel effectors.

With the introduction of the conceptual "Invasion Model", problems of the zigzag model were solved such as the depiction of MTI and ETI being displayed as being separated in time and space as well as the conceptual conflict that MAMPs are defined from the perspective of the plant while effectors are defined from the perspective of the invading microbe. The Invasion Model states that plants have evolved invasion pattern receptors (IPRs) to recognize microbial or modified-self ligands (termed invasion patterns (IPS) that may elicit an IP-triggered response (IPTR) with the intention to reveal invasion (8). Plant pathogens on the other hand can potentially suppress IPTRs by secreting effector proteins. An example for IP-IPR interaction can be found between the soil-borne pathogen Verticillium dahliae, that causes Verticillium wilt disease in over 200 dicotyledonous plant species $(9,10)$ and one of its host tomato. In tomato, the immune receptor $V e 1$ has been shown to mediate resistance to $V$. dahliae race 1 strains that secrete the effector VdAve1 (avirulence on Ve1 tomato) that is recognized as an IP (11). While it has been shown that VdAve1 perception activates Ve1-mediated resistance in tomato, Ave1 was also found to play an important role in disease, as Ave1 deficient $V$. dahliae mutants are hampered in virulence on tomato lines lacking Ve1 and on Arabidopsis (12).

Interestingly, successful IPTR signaling of the host, does not necessarily lead to the abolishment of infection (8). In fact, some plant pathogenic fungi deliberately evoke IPTRs in their hosts. For example, the necrotrophic fungus Parastagonospora nodorum, a wheat pathogen that causes Septoria nodorum blotch, has mastered this approach by recruiting IP-IPR interactions for cell death induction to establish disease $(13,14)$. Nine interactions are currently known between $P$. nodorum secreted IPs and corresponding wheat susceptibility genes/ IPRs (13, 15-28). Although fungi like P. nodorum appear to deliberately hijack IPTR to trigger cell death in the host, some pathogen-derived effectors can cause plant cell death solely due to their toxic nature. For 
example, a protein family with such a cytotoxic character are the Nep1-like proteins (NLPS) (29). Here, toxicity is hypothesized to be linked to cytolytic activity of the proteins resulting in plant plasma membrane depolarization and, subsequently, cell death (30-33). The wheat pathogen Zymoseptoria tritici, a hemibiotrophic Dothideomycete that causes septoria tritici blotch, for example harbors MgNLP whose product is able to cause cell death (34). However, MgNLP necrosis-inducing activity was characterized as selective as necrosis formation was observed in Arabidopsis and tobacco but not in wheat.

Besides effector proteins, secondary metabolites (SMS) are also well-known for their toxic properties. For instance, Alternaria spp. are omnipresent saprophytic or pathogenic fungi with a broad host range and are notorious producers of a variety of host-specific toxins that display diverse modes of action $(35,36)$. For example, the host-specific Alternaria toxins ACT-toxin, AFtoxin, and AK-toxin have a 9,10-epoxy-8-hydroxy-9-methyl-decatrienoic acid (EDA) backbone and are therefore members of the EDA family that have the potential to modify the plasma membrane of susceptible host cells (37-40). AK toxin I of the Alternaria alternata Japanese pear pathotype has been shown to induce plasma membrane modifications such as plasmalemmal invaginations, fusion of Golgi vesicles to invaginated plasma membranes, and accumulation of polysaccharides and membrane fragments derived from invaginated plasma membranes in susceptible pear plants (41-44). Associated with membrane modification was the formation of reactive oxygen species (ROS) that are hypothesized to be responsible for lipid peroxidation (42). Furthermore, irreversible depolarization of the plasma membrane results in subsequent electrolyte leakage which ultimately leads to host cell death $(35,39,40,44,45)$.

In contrast to host-specific toxins that require a corresponding molecular target to be present in their host in order to be effective, some fungi also produce toxins, termed non host-specific toxins that are almost universally toxic. For example, toxins belonging to the family of perylenequinones are considered to be non host-specific because they cause cell damage nearly universally to living cells (46-51). One of the most well-studied members of the perylenequinone family is cercosporin. Species in the genus Cercospora have been shown to rely on cercosporin to facilitate infection (52-54). As for all other perylenequinones, toxicity of this molecule can be traced back to the 3,10-dihydroxy-4,9-perylenequinone backbone, a common feature among all members of the perylenequinone family (Figure 1) (55-57). This chromophoric core allows absorption of light in the range of visible to UV light, leading to energetic excitation of the compound. Once cercosporin reaches an excited triplet state it reacts with oxygen leading to the formation of ROS. The emerging ROS have the capability to induce cell death of the host by causing lipid peroxidation and indiscriminate damage to proteins and DNA $(58,59)$. While cercosporin production has been demonstrated for Cercospora species and recently also for Colletorichum fioriniae (60), many other plant pathogenic fungi such as Cladosporium phlei, causal agent of leaf spot disease of timothy (61), or the citrus pathogen Elsinoë fawcettii (62) have been shown to produce structurally similar perylenequinones that share the same mode of action. 
<smiles>COc1c(C[C@H](C)O)c2c3c(C[C@H](C)O)c(OC)c(=O)c4c(O)cc5c6c(c(O)cc(c(c3-2)c1=O)c64)OCO5</smiles>

cercosporin

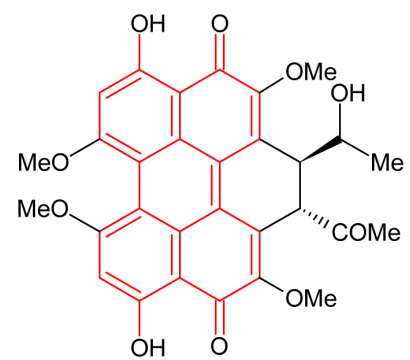<smiles>COc1c(OC)c2c3c4c(c(OC)cc(O)c4c1=O)c(OC)c(C[C@H](C)O)c(C[C@H](C)O)c-3c1c(OC)c(O)cc(O)c1c2=O</smiles>

phleichrome<smiles></smiles><smiles>COc1cc(O)c2c(OC)cc(O)c3c(=O)c(OC)c4c5c(c23)-c1c1c(O)cc(OC)c(c15)[C@@H](C(C)=O)[C@@H]4C(C)=O</smiles>

elsinochrome A

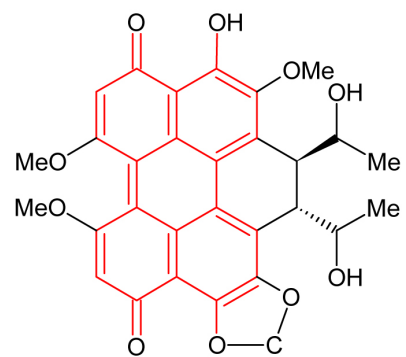

Figure 1. Structures of related perylenequinones. Cercosporin secreted by C. beticola, phleichrome by $C$. phlei and elsinochromes A, B, C, and D produced by E. fawcettii are structurally related as they share a common backbone (indicated in red). Structural differences between the molecules are mostly due to various side chains.

\section{Cercospora beticola - my pathogen of interest}

Cercospora beticola is a hemibiotrophic fungus that causes Cercospora leaf spot disease on sugar beet plant (Beta vulgaris), the most destructive foliar disease on sugar beet worldwide (63). In the field, C. beticola can over-winter as stromata in infected leftover leaf material until conidiospores are disseminated throughout the field by insects, wind, and water splash (63-65). On the sugar beet leaf, germinating spores enter the host through stomata to gain access to the apoplast where the fungus continues growing intercellularly $(63,66)$. While the initial phase of colonization is asymptomatic, small, circular necrotic spots will form when the fungus switches to its necrotrophic phase $(63,66,67)$. The increase in lesion number causes the lesions to fuse which leads to large necrotic tissue patches on the leaf where new conidia are formed. These conidia can again serve as inoculum for a new disease cycle. The utilization of effectors to facilitate disease has been demonstrated for many pathogenic fungi. However, the only C. beticola effector identified to-date is the secondary metabolite cercosporin. The ability to produce cercosporin is conserved in almost all Cercospora species and targeted gene replacement studies revealed that cercosporin contributes to virulence in multiple Cercospora species $(48,52-54)$.

\section{Thesis outline}

In this thesis, the effector repertoire of the fungal sugar beet pathogen Cercospora beticola was investigated. Additionally, I extended my studies to other fungal pathogens that have been 
identified to produce toxins that are structurally-related and therefore belong to the same toxin family as to cercosporin produced by C. beticola.

Plant pathogenic fungi utilize effectors to promote virulence during colonization of the host plant. These effectors often have diverse modes of action and can be derived from proteins or secondary metabolites. Chapter 2 gives an overview of the broad diversity of known fungal virulence mechanisms.

The effector protein VdAve1 (Verticillium dahliae Avirulence on Ve1 tomato) was originally identified as a race 1 specific effector protein of the soil-borne pathogen Verticillium dahliae. In tomato, resistance to $V$. dahliae race 1 strains is conferred by the extracellular leucine-rich repeat cell surface receptor Ve1. While homologs of VdAve1 can primarily be found in plants, some plant pathogenic fungi also harbor homologs of VdAve1 such as Fusarium oxysporum (FoAve1), Colletotrichum higginsianum (ChAve1) and Cercospora beticola (CbAve1). In Chapter 3, we determined the gene expression profiles of CbAve1, FoAve1, and ChAve1 during host infection by the producing pathogen and examined whether these genes are necessary for full virulence of their producer.

As a hemibiotrophic fungus, C. beticola relies on host cell death to provide nutrients during the necrotrophic stage of the lifecycle. Therefore, we hypothesized that the fungus secretes effector proteins during infection that facilitate disease by causing necrosis formation. Chapter 4 describes a phenotype-based approach that aimed to identify conditions in which the fungus produces effector proteins in vitro. The expression profile of the effector candidate CbNip1 during C. beticola infection was determined and the candidate was heterologously expressed in E. coli for functional analysis. Furthermore, contribution of the candidate to $C$. beticola virulence was assessed.

Apart from proteinaceous effectors C. beticola is a well-known producer of phytotoxic secondary metabolites. During infection, C. beticola produces the perylenequinone cercosporin. This secondary metabolite is formed via a polyketide synthesis gene cluster that for decades was thought to consist of eight genes. However, Chapter 5 describes the identification of additional cercosporin biosynthesis genes. Targeted gene replacement of novel cercosporin biosynthesis genes aimed to determine their involvement in toxin formation. Furthermore, orthologous cercosporin clusters in other Ascomycetes were identified through phylogenetic analysis.

Due to high structural similarity of perylenequinone family members, it was hypothesized that the biosynthetic gene clusters responsible for perylenequinone formation display considerable similarity between species. Chapter 6 utilizes gene cluster conservation to facilitate the identification of perylenequinone and DHN-melanin biosynthesis pathways in multiple fungal species.

Finally, Chapter 7 discusses the major findings described in this thesis and provides an overview of the effector repertoire of C. beticola at its current state. 


\section{References}

1. Flor HH (1942) Inheritance of pathogenicity in melampsora lini. Phytopathology 32:653-669.

2. Boller T (1995) Chemoperception of microbial signals in plant cells. Annual review of plant biology 46(1):189-214.

3. Zipfel C, et al. (2004) Bacterial disease resistance in Arabidopsis through flagellin perception. Nature 428:764.

4. Gómez-Gómez L \& Boller T (2000) FLS2: An LRR receptor-like kinase involved in the perception of the bacterial elicitor flagellin in Arabidopsis. Molecular Cell 5(6):1003-1011.

5. Jones JD \& Dangl JL (2006) The plant immune system. Nature 444(7117):323-329.

6. Zipfel C (2008) Pattern-recognition receptors in plant innate immunity. Current Opinion in Immunology 20(1):10-16.

7. Chisholm ST, Coaker G, Day B, \& Staskawicz BJ (2006) Host-microbe interactions: Shaping the evolution of the plant immune response. Cell 124(4):803-814.

8. Cook DE, Mesarich $\mathrm{CH}$, \& Thomma BP (2015) Understanding plant immunity as a surveillance system to detect invasion. Annual review of phytopathology 53:541-563.

9. Fradin EF \& Thomma BPHJ (2006) Physiology and molecular aspects of verticillium wilt diseases caused by V. dahliae and V. albo-atrum. Molecular Plant Pathology 7(2):71-86.

10. Agrios G (1997) Plant pathology, 4" edition (Academic Press. San Diego. California).

11. Fradin EF, et al. (2009) Genetic dissection of verticillium wilt resistance mediated by tomato Ve1. Plant physiology 150(1):320-332.

12. de Jonge R, et al. (2012) Tomato immune receptor Ve1 recognizes effector of multiple fungal pathogens uncovered by genome and RNA sequencing. Proceedings of the National Academy of Sciences 109(13):5110-5115.

13. Shi G, et al. (2016) The hijacking of a receptor kinase-driven pathway by a wheat fungal pathogen leads to disease. Science advances 2(10):e1600822.

14. Friesen TL, Faris JD, Solomon PS, \& Oliver RP (2008) Host-specific toxins: Effectors of necrotrophic pathogenicity. Cellular microbiology 10(7):1421-1428.

15. Liu Z, et al. (2004) Genetic and physical mapping of a gene conditioning sensitivity in wheat to a partially purified host-selective toxin produced by Stagonospora nodorum. Phytopathology 94(10):1056-1060.

16. Liu ZH, et al. (2004) Quantitative trait loci analysis and mapping of seedling resistance to Stagonospora nodorum leaf blotch in wheat. Phytopathology 94(10):1061-1067.

17. Friesen $T L$, et al. (2006) Emergence of a new disease as a result of interspecific virulence gene transfer. Nature Genetics 38:953.

18. Liu Z, et al. (2006) The Tsn1-ToxA interaction in the wheat-Stagonospora nodorum pathosystem parallels that of the wheat-tan spot system. Genome 49(10):1265-1273.

19. Friesen TL, Meinhardt SW, \& Faris JD (2007) The Stagonospora nodorum-wheat pathosystem involves multiple proteinaceous host-selective toxins and corresponding host sensitivity genes that interact in an inverse genefor-gene manner. The Plant Journal 51(4):681-692.

20. Friesen TL, Zhang Z, Solomon PS, Oliver RP, \& Faris JD (2008) Characterization of the interaction of a novel Stagonospora nodorum host-selective toxin with a wheat susceptibility gene. Plant physiology 146(2):682-693.

21. Abeysekara NS, Faris JD, Chao S, McClean PE, \& Friesen TL (2011) Whole-genome QTL analysis of Stagonospora nodorum blotch resistance and validation of the SnTox4-Snn4 interaction in hexaploid wheat. Phytopathology 102(1):94-104.

22. Abeysekara NS, Friesen TL, Keller B, \& Faris JD (2009) Identification and characterization of a novel host-toxin interaction in the wheat-Stagonospora nodorum pathosystem. Theoretical and Applied Genetics 120(1):117-126.

23. Friesen TL, et al. (2009) Host-selective toxins produced by Stagonospora nodorum confer disease susceptibility in adult wheat plants under field conditions. Theoretical and Applied Genetics 118(8):1489-1497.

24. Chu C-G, Faris JD, Xu SS, \& Friesen TL (2010) Genetic analysis of disease susceptibility contributed by the compatible Tsn1-SnToxA and Snn1-SnTox1 interactions in the wheat-Stagonospora nodorum pathosystem. Theoretical and Applied Genetics 120(7):1451-1459

25. Zhang Z, et al. (2011) Two putatively homoeologous wheat genes mediate recognition of SnTox3 to confer effector-triggered susceptibility to Stagonospora nodorum. The Plant Journal 65(1):27-38.

26. Friesen TI, Chu C, Xu SS, \& Faris JD (2012) SnTox5-Snn5: A novel Stagonospora nodorum effector-wheat gene interaction and its relationship with the SnToxA-Tsn1 and SnTox3-Snn3-b1 interactions. Molecular Plant Pathology 13(9):1101-1109.

27. Gao Y, et al. (2015) Identification and characterization of the SnTox6-Snn6 interaction in the Paratagonospora nodorum-wheat pathosystem. Molecular Plant-Microbe Interactions 28(5):615-625.

28. Shi G, et al. (2015) The wheat Snn7 gene confers susceptibility on recognition of the Parastagonospora nodorum necrotrophic effector SnTox7. The Plant Genome 8(2).

29. Oome S, et al. (2014) Nep1-like proteins from three kingdoms of life act as a microbe-associated molecular pattern in Arabidopsis. Proceedings of the National Academy of Sciences 111(47):16955-16960.

30. Böhm H, et al. (2014) A conserved peptide pattern from a widespread microbial virulence factor triggers pattern-induced immunity in Arabidopsis. PLOS Pathogens 10(11):e1004491.

31. Albert l, et al. (2015) An RLP23-SOBIR1-BAK1 complex mediates NLP-triggered immunity. Nature Plants 1:15140.

32. Zaparoli G, et al. (2011) The crystal structure of necrosis- and ethylene-inducing protein 2 from the causal agent of cacao's witches' broom disease reveals key elements for its activity. Biochemistry 50 (45):9901-9910. 
33. Ottmann C, et al. (2009) A common toxin fold mediates microbial attack and plant defense. Proceedings of the National Academy of Sciences 106(25):10359-10364.

34. Motteram J, et al. (2009) Molecular characterization and functional analysis of MgNLP, the sole NPP1 domaincontaining protein, from the fungal wheat leaf pathogen Mycosphaerella graminicola. Molecular plant-microbe interactions 22(7):790-799

35. Tsuge T, et al. (2013) Host-selective toxins produced by the plant pathogenic fungus Alternaria alternata. FEMS Microbiology Reviews 37(1):44-66.

36. Thomma BPHJ (2003) Alternaria spp.: From general saprophyte to specific parasite. Molecular Plant Pathology 4(4):225-236.

37. Nakashima T, et al. (1985) Isolation and structures of AK-toxin I and II, host-specific phytotoxic metabolites produced by Alternaria alternata japanese pear pathotype. Agricultural and Biological Chemistry 49(3):807-815.

38. Nakatsuka S-i, et al. (1986) Structure of af-toxin II, one of the host-specific toxins produced by Alternaria alternata strawberry pathotype. Tetrahedron Letters 27(24):2753-2756.

39. Kohmoto K, et al. (1993) Isolation and biological activities of two host-specific toxins from the tangerine pathotype of Alternaria alternata. Phytopathology 83(5):495-502.

40. Otani H, et al. (1985) Biological activities of AK-toxins I and II, host-specific toxins from Alternaria alternata japanese pear pathotype. Japanese Journal of Phytopathology 51(3):285-293.

41. Suzuki T, Shinogi T, Unno K, Narusaka Y, \& Park P (2002) B-1,3-D-glucan transported from Golgi apparatus of japanese pear leaves is a component of extracellular polysaccharides accumulated after AK-toxin I treatment. Journal of General Plant Pathology 68(4):267-276.

42. Shimizu N, et al. (2006) Reactive oxygen species (ROS) generation and ROS-induced lipid peroxidation are associated with plasma membrane modifications in host cells in response to AK-toxin I from Alternaria alternata japanese pear pathotype. Journal of General Plant Pathology 72(1):6-15.

43. Park P, Fukutomi M, Akai S, \& Nishimura S (1976) Effect of the host-specific toxin from Alternaria kikuchiana on the ultrastructure of plasma membranes of cells in leaves of japanese pear. Physiological Plant Pathology 9(2):167-174.

44. Park P, Ohno T, Nishimura S, Kohmoto K, \& Otani H (1987) Leakage of sodium ions from plasma membrane modification, associated with permeability change, in host cells treated with a host-specific toxin from a japanese pear pathotype of Alternaria alternata. Canadian Journal of Botany 65(2):330-339.

45. Maekawa N, et al. (1984) Studies on host-specific af-toxins produced by Alternaria alternata strawberry pathotype causing Alternaria black spot of strawberry. Japanese Journal of Phytopathology 50(5):600-609.

46. Favilla M, Macchia L, Gallo A, \& Altomare C (2006) Toxicity assessment of metabolites of fungal biocontrol agents using two different (Artemia salina and Daphnia magna) invertebrate bioassays. Food and Chemical Toxicology 44(11):1922-1931.

47. Tamaoki T \& Nakano H (1990) Potent and specific inhibitors of protein kinase C of microbial origin. Bio/ Technology 8:732.

48. Daub ME \& Ehrenshaft M (2000) The photoactivated Cercospora toxin cercosporin: Contributions to plant disease and fundamental biology. Annual review of phytopathology 38(1):461-490.

49. Vandenbogaerde AL, et al. (1998) Cytotoxicity and antiproliferative effect of hypericin and derivatives after photosensitization. Photochemistry and Photobiology 67(1):119-125.

50. Chung K-R (2011) Elsinoë fawcettii and Elsinoë australis: The fungal pathogens causing citrus scab. Molecular Plant Pathology 12(2):123-135.

51. Stergiopoulos I, Collemare J, Mehrabi R, \& De Wit PJ (2012) Phytotoxic secondary metabolites and peptides produced by plant pathogenic Dothideomycete fungi. FEMS microbiology reviews 37(1):67-93.

52. Weiland JJ, Chung K-R, \& Suttle JC (2010) The role of cercosporin in the virulence of Cercospora spp. To plant hosts. Cercospora leaf spot of sugar beet and related species, eds Lartey RT, Weiland JJ, Panella L, Crous PW, \& Windels CE (APS Press, St. Paul).

53. Choquer $M$, et al. (2005) The CTB1 gene encoding a fungal polyketide synthase is required for cercosporin biosynthesis and fungal virulence of Cercospora nicotianae. Molecular plant-microbe interactions 18(5):468-476.

54. Callahan TM, Rose MS, Meade MJ, Ehrenshaft M, \& Upchurch RG (1999) CFP, the putative cercosporin transporter of Cercospora kikuchii, is required for wild type cercosporin production, resistance, and virulence on soybean. Molecular plant-microbe interactions 12(10):901-910.

55. Guedes RC \& Eriksson LA (2007) Photophysics, photochemistry, and reactivity: Molecular aspects of perylenequinone reactions. Photochemical \& Photobiological Sciences 6(10):1089-1096.

56. Foote CS (1968) Mechanisms of photosensitized oxidation. Science 162(3857):963-970.

57. DeRosa MC \& Crutchley RJ (2002) Photosensitized singlet oxygen and its applications. Coordination Chemistry Reviews 233:351-371.

58. Birben E, Sahiner UM, Sackesen C, Erzurum S, \& Kalayci O (2012) Oxidative stress and antioxidant defense. World Allergy Organization Journal 5(1):9.

59. Blokhina O, Virolainen E, \& Fagerstedt KV (2003) Antioxidants, oxidative damage and oxygen deprivation stress: A review. Annals of botany 91(2):179-194.

60. de Jonge R, et al. (2018) Gene cluster conservation provides insight into cercosporin biosynthesis and extends production to the genus Colletotrichum. Proceedings of the National Academy of Sciences, doi:10.1073/ pnas.1712798115. 
61. Yoshihara T, Shimanuki T, Araki T, \& Sakamura S (1975) Phleichrome; a new phytotoxic compound produced by Cladosporium phlei. Agricultural and Biological Chemistry 39(8):1683-1684.

62. Weiss U, Flon H, \& Burger WC (1957) The photodynamic pigment of some species of Elsinoë and Sphaceloma. Archives of biochemistry and biophysics 69:311-319.

63. Weiland J \& Koch G (2004) Sugarbeet leaf spot disease (Cercospora beticola sacc.). Molecular plant pathology 5(3):157-166.

64. Khan MFR \& Khan J (2010) Survival, spore trapping, dispersal, and primary infection site for Cercospora beticola in sugar beet. Cercospora leaf spot of sugar beet and related species, eds Lartey RT, Weiland JJ, Panella L, Crous PW, \& Windels CE (APS Press, St. Paul).

65. Solel Z \& Minz G (1971) Infection process of Cercospora beticola in sugarbeet in relation to susceptibility. Phytopathology 61:463-466.

66. Steinkamp MP, Martin SS, Hoefert LL, \& Ruppel EG (1979) Ultrastructure of lesions produced by Cercospora beticola in leaves of Beta vulgaris. Physiological Plant Pathology 15(1):13-26.

67. Weltmeier F, et al. (2011) Transcript profiles in sugar beet genotypes uncover timing and strength of defense reactions to Cercospora beticola infection. Molecular Plant-Microbe Interactions 24(7):758-772. 



\section{Tools of the crook - infection strategies of fungal plant pathogens}

Luis Rodriguez-Moreno*, Malaika K. Ebert*, Melvin D. Bolton\#,

and Bart P.H.J. Thomma\# (2018) Tools of the crook - infection strategies of

fungal plant pathogens. The Plant Journal 93, 664-674; doi: 10.1111/tpj.13810

* These authors contributed equally

\#These authors contributed equally 


\section{Abstract}

Fungi represent an ecologically diverse group of microorganisms that includes plant pathogenic species able to cause considerable yield loses in crop production systems worldwide. In order to establish compatible interactions with their hosts, pathogenic fungi rely on the secretion of molecules of diverse nature during host colonization to modulate host physiology, manipulate other environmental factors or provide self-defence. These molecules, collectively known as effectors, are typically small secreted cysteine-rich proteins, but may also comprise secondary metabolites and sRNAs. Here, we discuss the most common strategies that fungal plant pathogens employ to subvert their host plants in order to successfully complete their life cycle and secure the release of abundant viable progeny. 


\section{Introduction}

Fungi constitute an evolutionarily and ecologically diverse group of microorganisms that includes plant pathogenic species that cause considerable yield losses in agricultural production systems worldwide. Generally, the lifestyles of plant pathogenic fungi are differentiated depending on the strategies used to acquire nutrients from their hosts. As such, obligate biotrophic fungi comprise those species that can only feed on living host tissue to meet their nutritional requirements and complete their life cycle. At the complete opposite of the spectrum, necrotrophic fungi trigger cell death in the host to secure nutrient supply. In between these extremes is a wide array of hemibiotrophic fungi that start their compatible host interaction with an initial biotrophic phase that, at one point in time when the infection progressed sufficiently, is followed by a transition to a necrotrophic stage. A parasitic life style that involves the extraction of sugars from other organisms is one of the ways in which non-heterotrophic organisms compensate for the inability to generate sugars through photosynthesis. Many biotrophic and hemibiotrophic fungi evolved haustoria, appendages of fungal hyphae that invaginate the host plasma membrane and grow inside host cells, to obtain these nutrients. Recently, it was demonstrated that the obligate biotrophic powdery mildew fungus Golovinomyces cichoracerum requires lipids for colonization that it receives from the host plant (1).

Lifestyle differences largely determine the wide array of strategies that fungi use to evade, counteract or hijack plant defences in their effort to complete their life cycle and secure the production of viable progeny. Irrespective of their life style, microbial pathogens are all believed to utilize so-called effectors, in planta-secreted molecules of various nature, to support host colonization, often, but not exclusively, through suppression of host immune responses (2). Over the years it has become evident that haustoria are not only fungal feeding structures, but are also active sites for secretion and translocation of effectors into the host (3-6).

Plants have developed an innate immune system to recognize and respond to microbes (79) (Figure 1). This immune system relies on the presence of immune receptors that detect pathogen invasion through sensing of pathogen(-induced) ligands, collectively termed invasion patterns, to mount appropriate immune responses (9). Recognition of invasion patterns triggers both local and systemic reactions to respond in a quick and focussed manner to attempted microbial ingress (7-9). For example, the well-characterized invasion pattern chitin, an important constituent of fungal cell walls, is recognized by plants through plasma membrane-localized extracellular lysin motif (LysM)-containing receptor molecules (10, 11). Pathogen recognition by plant immune receptors causes ion fluxes, the accumulation of reactive oxygen species (ROS), and a quick activation of defence-related mitogen-activated protein kinase (MAPKs) cascades that cause an extensive transcriptional reprogramming of the host (12-14). Furthermore, pathogen perception leads to reinforcement of plant cell walls by callose deposition, changes in hormone biosynthesis, and the production of antimicrobial compounds (15). In many cases, these defence responses collectively are sufficient to render the interaction between the plant and the invader incompatible, implying that pathogen ingress is halted or at least significantly slowed down. However, co-evolutionary processes have selected pathogens that employ a plethora of virulence strategies to overcome various mechanisms within plant immune systems. 
In this review, we summarize the different virulence strategies that plant pathogenic fungi use to subvert their hosts. While there are excellent reviews that discuss individual strategies in detail, the aim of this review is to outline the broad diversity of known fungal virulence mechanisms (Figure 2).

\section{Fungal strategies for host penetration}

One of the first barriers that fungal pathogens have to breach to gain entrance to their hosts is cell walls that are mainly composed of carbohydrates. Many plant pathogenic fungi utilize specialized infection structures, called appressoria, to generate focused turgor pressure to breach the cell wall by force (16). Depending on the fungal species, the turgor pressure is combined with the localized release of cell wall-degrading enzymes (CWDEs) (16). Furthermore, effectors are secreted from appressorial penetration pores prior to host invasion (17).

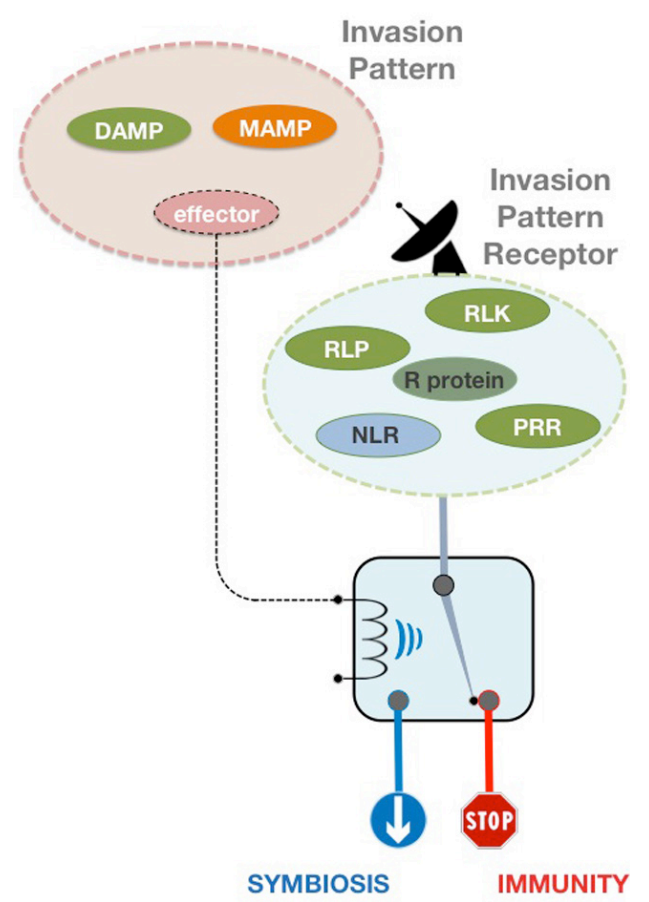

Figure 1. Schematic representation of the "Invasion Model" to describe the molecular basis of plant immunity against fungal pathogens. In this model invasion pattern receptors, comprising any type of host receptor, detect invasion patterns, comprising externally encoded and modified-self ligands that announce invasion, to mount an effective immune response and halt the symbiosis. Fungal effectors may manipulate the induced response to tweak the symbiosis to their benefit.
Fungi typically produce an arsenal of so-called carbohydrate-active enzymes (CAZymes) that are grouped into five enzyme classes, namely glycoside hydrolases, glycosyltransferases, polysaccharide lyases, carbohydrate esterases, and redox enzymes with auxiliary activities (18). Several of the polysaccharide lyases, glycoside hydrolases, and carbohydrate esterases are known as CWDEs that are used to degrade host cell walls. Typically plant pathogenic species contain higher numbers of CAZyme genes than saprophytic and animal pathogenic strains (19). Whereas obligate biotrophs typically lack extensive catalogs of CWDE genes and likely only use such enzymes for subtle manipulations of host cell walls such as at the cellular entrance sites for haustoria, necrotrophic fungi were often thought of as 'brute-force' pathogens that rely on large CWDE catalogs to macerate host cell walls and initiate colonization (20). These enzymes occur in multiple isoforms that not only differ in isoelectric point and molecular weight, but also in timing of their production and processing, offering especially broad host-range necrotrophs particular flexibility to penetrate and colonize their hosts. Besides colonization, these enzymes also liberate nutrients for the pathogen. For example, 
hydrolysis of pectin by fungal pectinases weakens the cell wall to enable penetration while also providing the fungus with important carbon sources for growth (21). Indeed, strategies to limit pectin degradation were explored by generating transgenic wheat lines expressing pectin methyl esterase inhibitors, which exhibited altered pectin methyl esterification that resulted in reduced activity of pathogen pectic enzymes and reduced disease from hemibiotrophic pathogens Fusarium graminearum and Bipolaris sorokiniana (22). Similarly, wheat lines expressing genes encoding a xylanase inhibitor and polygalacturonase inhibiting protein exhibited increased resistance to Fusarium head blight (23). However, F. graminearum single gene deletion mutants for polygalacturonase or xylanase resulted in minor effects on virulence, while double gene mutants were significantly reduced in virulence on soybean and wheat plants, highlighting the synergism between CWDEs (24).

Besides plant cell wall-degrading enzymes, fungi secrete CWDEs to modulate their own cell walls and accommodate morphological changes. It was recently proposed that such activity facilitates pathogenesis of plants by enabling host colonization. A glycosyltransferase enzyme from the hemibiotrophic wheat pathogen Zymoseptoria tritici was reported to enable hyphal growth on solid surfaces that is essential for fungal disease of wheat plants (25). Homologs of this particular enzyme are widespread in fungi, and mutants in the taxonomically unrelated $F$. graminearum were similarly impaired in hyphal growth and pathogenicity (25).

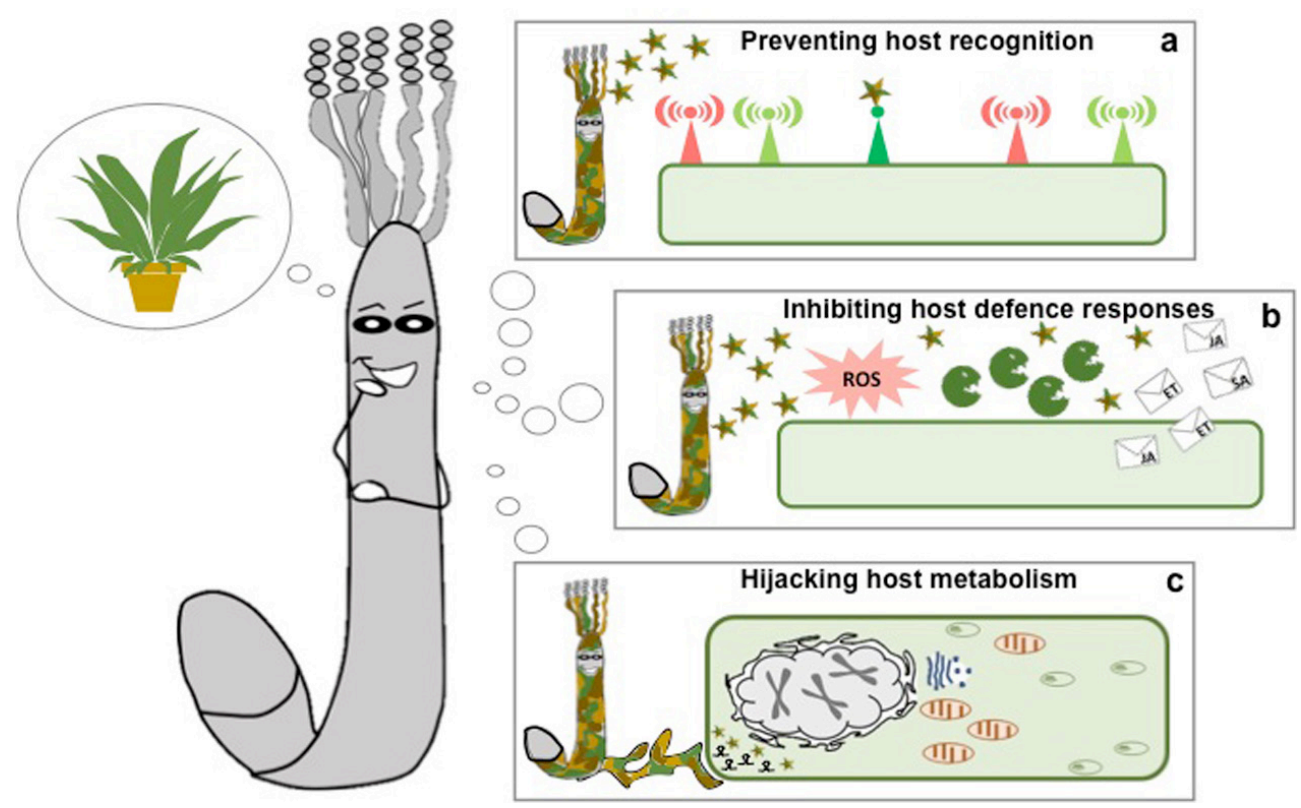

Figure 2. Illustration of fungal pathogen strategies to surmount host plants. (a) Secretion of effectors that perturb recognition by plant immune receptors. (b) Secretion of effectors that subvert plant defense responses that are induced upon pathogen detection. (c) Delivery of various types of molecules to hijack host metabolism. 


\section{Fungal strategies preventing plant recognition}

Plants evolved a plethora of plasma membrane-localized immune receptors for surveillance of the extracellular space for pathogen(-induced) ligands $(13,15,26)$. The perception of these ligands is relayed into downstream signalling events which lead to the activation of plant defences $(14,26)$. Structural components of the fungal cell wall, such as glucans and chitin, are typically recognised as pathogen ligands by plant receptors $(10,11,27,28)$. As part of their defence system, plants secrete glucanases and chitinases to compromise the integrity of fungal cell walls and release oligomeric fragments that can act as ligand for extracellular immune receptors (29). Fungi have evolved several strategies to overcome host immune responses that involve fungal cell walls, including alterations in cell wall compositions and the secretion of effectors to protect cell walls or perturb recognition of cell wall components $(2,29)$.

Magnaporthe oryzae is a hemibiotrophic fungal pathogen and causal agent of rice blast disease (30). During infection, M. oryzae responds to the epidermal wax component 1,16-hexadecanediol by accumulating a-1,3-glucans at the surface of the cell wall, resulting in inhibition of chitin degradation by plant chitinases (31). Accordingly, mutants that are unable to accumulate a-1,3-glucans at the fungal cell surface trigger rapid activation of host defences (31). A similar strategy has been reported for the maize pathogen Colletotrichum graminicola that modifies the $\beta$-glucan composition of its biotrophic hyphae, as the content of $\beta-1,3-$ and $\beta-1,6$ glucans is significantly reduced when compared with appressoria and necrotrophic hyphae (3234). Hence, C. graminicola strains that overexpress a $\beta$-1,3-glucan synthase in their biotrophic hyphae induce stronger host defense responses and display reduced virulence (32). However, C. graminicola strains that are unable to produce $\beta-1,6$-glucans are defective in appressorium formation and thus non-pathogenic (34).

Modification of cell walls is not the only strategy employed by fungal pathogens to prevent plant recognition. For instance, the tomato leaf mould fungus Cladosporium fulvum secretes the carbohydrate-binding effector protein Ecp6 that suppresses chitin-triggered host immunity. The chitin-binding capacity of Ecp6 is mediated by three Lysin motifs (LysMs) $(35,36)$ that occur in proteins of a wide range of organisms to confer the ability to bind various types of polysaccharides, including peptidoglycan and chitin, through a conserved ßaaß-fold (37). Interestingly, two out of the three LysM domains of Ecp6 cooperate to form a groove that binds chitin fragments with ultra-high (pM) affinity that allows to outcompete host receptors for chitin binding (36). Besides Ecp6, C. fulvum also secretes the chitin binding effector molecule Avr4 during host colonization. As opposed to LysMs, Avr4 binds chitin through an invertebrate chitinbinding module to protect the cell wall against hydrolysis by host enzymes $(38,39)$. In contrast to Avr4 homologs that only occur in a limited set of fungi that are closely related to C. fulvum (40), LysM effector proteins occur in a wide variety of fungi (41), and have been shown to suppress chitin-triggered immunity on various plant hosts such as for $Z$. tritici on wheat (42), for M. oryzae on rice (43), for C. higginsianum on Arabidopsis (44) and for $V$. dahliae on tomato (45).

Whereas chitin perception in plants is relatively well-understood (46), $\beta$-glucan perception and signalling mechanisms remain poorly characterized (47). The root endophyte Piriformospora 
indica secretes the $\beta$-glucan-binding lectin effector FGB1 that suppresses $\beta$-glucan-triggered host immunity (48). Prevention of $\beta$-glucan detection by the plant seems important for successful fungal infection as overexpression of the Piriformospora indica FGB1 homolog in Ustilago maydis was shown to lead to an increase in virulence. Interestingly, FGB1 homologs are widespread in fungi (48).

A further strategy to protect fungal cell walls and prevent detection of cell wall components is through the secretion of proteases that affect hydrolytic host enzymes (49). Fungal chitinasemodifying proteins (CMPs) have been reported in several maize pathogens including Bipolaris zeicola (50), Stenocarpella maydis (51) and Fusarium verticilloides (52). Similarly, F. oxysporum f. sp. lycopersici, Verticillium dahliae and Botrytis cinerea were found to secrete CMPs that can degrade extracellular tomato chitinases (53).

\section{Fungal strategies for inhibiting host defence responses}

Pathogen recognition by plants results in a panoply of defence responses to hamper pathogen invasion. These responses comprise swift ion fluxes, $\mathrm{pH}$ changes, production of reactive oxygen species (ROS), but also the production of local and systemic signalling molecules and of antimicrobial compounds. Various mechanisms are employed by fungal pathogens to subvert such responses.

\section{Subverting ROS damage}

ROS production is mostly due to the activity of membrane bound NADPH-oxidases and cell-wall associated peroxidases (POX) (54-56). While relatively low concentrations of ROS have been reported to act as defence signalling molecules, (57-59), high concentrations of ROS are extremely harmful to cells as they have been shown to cause oxidative damage $(60,61)$. The apoplastic effector Pep1 of the biotrophic maize pathogen Ustilago maydis accumulates at sites where biotrophic hyphae move from cell to cell in maize tissue to inhibit the oxidative burst through inhibition of POX12, a type-III class heme-peroxidase that is highly induced after $U$. maydis penetration $(62,63)$. Pep1 only causes partial inhibition of the maize apoplastic peroxidase activity, suggesting that not all peroxidase-producing enzymes in the maize apoplast are targeted by Pep1 (63).

\section{Manipulating tissue $\mathrm{pH}$}

Many fungal pathogens induce a pH shift in the host tissue surrounding the infection site (64). For instance, S. sclerotiorum causes acidification of the infection area through the production of oxalic acid, leading to rapid death of host tissues (20). However, other pathogens induce alkalinisation of host tissue $(65,66)$. During host colonisation, the vascular wilt pathogen $F$. oxysporum causes an increase of the extracellular $\mathrm{pH}$ from about 5 to 7 through the secretion of a peptide with homology to plant rapid alkalinizing factors (RALFs) $(66,67)$. Interestingly, F. oxysporum strains that are no longer able to produce this peptide trigger enhanced host defence, indicating a role in suppression of host immunity. Although this role in virulence has been challenged (68), RALF-encoding genes can be found in many fungal pathogens, suggesting a universal mechanism to alkalinize infection sites to suppress host immunity $(66,68)$. 
Inhibition of host proteases

Many of the molecules that fungal pathogens secrete in order to establish the parasitic interaction with their hosts are of proteinaceous nature, and hence plants secrete proteases to undermine this pathogen strategy $(69,70)$. The apoplast of tomato and Arabidopsis contains various proteases that contribute to host defence $(71,72)$. Among these, the tomato apoplast contains the extracellular cysteine protease Rcr3 that plays a central role in resistance mediated by the Cf-2 immune receptor of tomato and that is activated by the C. fulvum effector Avr2 (73). The Avr2 effector inhibits the activity of Rcr3, likely causing a conformational change in the Rcr3 structure that is recognized by Cf-2 $(73,74)$. Besides Rcr3, Avr2 inhibits various other host proteases that are required for pathogen defence (71). Other fungal pathogens also produce protease effectors to inhibit host proteases, such as the $U$. maydis Pit2 effector $(75,76)$.

\section{Subverting hormone signalling}

Plant growth and their responses to environmental cues, including pathogens, are largely governed by phytohormones. Typically, salicylic acid (SA) signalling governs resistance against biotrophic pathogens whereas a combination of jasmonic acid (JA) and ethylene (ET) signalling activates resistance against necrotrophic pathogens $(8,77,78)$. To a large extent, these signalling pathways act antagonistically and their balance needs to be governed carefully. Thus it is not surprising that pathogens evolved various strategies to affect phytohormone signalling. For instance, U. maydis secretes the chorismate mutase Cmu1 into host cells to perturb SA production by affecting the production of its precursor (79). Likely, Cmu1 acts in combination with the maize chorismate mutase $\mathrm{Cm} 1$ to increase the flow of chorismate from the plastid to the cytosol to diminish the available substrate for salicylic acid biosynthesis in plastids in turn (79). Furthermore, U. maydis produces Shy1, a salicylate hydroxylase that degrades SA during host invasion (80). Together these results suggest that perturbation of SA-mediated immunity is crucial for $U$. maydis colonization. Chorismate mutases have been identified in many eukaryotic plant pathogens pointing towards a common strategy for host manipulation. Similar to U. maydis, also V. dahliae has been proposed to target SA biosynthesis by secreting effectors with isochorismatase activity to hydrolyse isochorismate (81). Besides targeting SA signalling, fungal effectors that target JA signalling or ET signalling have been described as well $(82,83)$. For instance, the beneficial fungus Laccaria bicolor produces the Mycorrhiza-induced small secreted protein-7 (MiSSP7) during the interaction with its host Populus trichocarpa (83). Intriguingly, MiSSP7 interacts with the plant JASMONATE ZIM-DOMAIN (JAZ)-6 protein to provoke blockage of the expression of JA-inducible genes in the host to promote fungal colonization (83).

Besides the capacity to manipulate hormone balances in plant tissues, particular fungi appear have the ability to produce hormone-mimicking compounds to promote host colonization (8487). For instance, Fusarium pseudograminearum produces cytokinin-like molecules that activate plant cytokinin signaling to reprogram the host (88).

\section{The molecules that do the job: fungal effectors}

Typically, fungal effectors are described as small secreted, cysteine-rich proteins that are produced during host invasion (89). These fungal effectors can be divided into two types based on their 
extra- or intracellular localization in the host. Yet, how cytoplasmic effectors are translocated into host cells remains poorly understood (90-92). Nevertheless, two distinct secretion systems to target effectors have been described for M. oryzae. Cytoplasmic effectors accumulate in a socalled biotrophic interfacial complex, a plant membrane-rich structure associated with invasive hyphae that involves exocyst and t-SNARE components $(93,94)$. By contrast, apoplastic effectors are secreted from invasive hyphae via conventional secretion. In addition to proteinaceous effector molecules, other types of molecules are secreted by fungi with the aim to establish the parasitic relationship that therefore qualify to be labelled as effectors just as well.

\section{Secondary metabolites}

Secondary metabolites (SMs) are small bioactive molecules that often play crucial roles in the establishment of specific ecological niches but, unlike primary metabolites, are not essential for fungal growth, development, or reproduction. While fungal SMs are often known and valued for their anti-microbial activities, many fungi employ SMs to promote virulence. Traditionally SMs involved with virulence are classified as either host-specific toxins (HSTs; discussed below), because they have specific targets in the host, or non-HSTs that typically do not have a specific host target and are generally toxic to a wide-range of organisms including the host instead (95). Perylenequinones, for example, are a family of photosensitizing SMs for which the mode of action is well-studied. The most prominent member of the family is cercosporin. This light-activated toxin is produced by most Cercospora spp. and has a very broad toxicity range to many organisms including plants, animals, bacteria and most fungi. Due to its photosensitizing nature, cercosporin is able to absorb light energy and subsequently react with oxygen (96). Products of this reaction are ROS that can cause protein and DNA damage and lipid peroxidation and eventually lead to cell death of the host $(97,98)$. As necrosis development lays the ground for fungal spore formation, it is speculated that cercosporin secretion might facilitate cell wall breaching to enable conidiophore and conidia production (99). The cercosporin biosynthesis gene cluster was recently shown to be found wide-spread in the Colletotrichum genus, implicating the role of cercosporin as a virulence factor in an important group of fungal plant pathogens (100).

\section{Host-selective toxins}

HSTs are known to induce necrotic host tissue reactions to promote host susceptibility (95). The effectiveness of HSTs depends on whether a plant possesses a corresponding toxin target, which may also define the host range of the producing pathogen. For example, maize lines harboring Texas cytoplasm for male sterility (Tcms) display extreme sensitivity to T-toxin and PM-toxin secreted by Cochliobolus heterostrophus race T and Mycosphaerella zeae-maydis, respectively $(95,101,102)$. Here, host susceptibility is conferred by a single plant gene T-urf13 that encodes URF13, a mitochondrial membrane protein to which either toxin can directly bind. Binding triggers URF13 to experience a conformational change which in turn results in the formation of a pore in the mitochondrial membrane. The ability to produce T-toxin is relevant for fungal virulence, as $C$. heterostrophus race $O$, a natural T-toxin lacking race and Tox1-deficient mutants of race $T$ show reduced virulence on Tcms carrying maize (103). Similarly, PM-toxin deficient Tox- mutants of M. zeae-maydis lost the ability to infect Tcms maize (104). Besides mitochondria, 
HSTs are also reported to target enzymes or other plant cell organelles like plasma membrane, chloroplast, endoplasmatic reticulum, nucleus, vacuole, and Golgi bodies with the objective to suppress host defense responses and/or induce host cell death (105).

Another HST toxin is victorin, a family of related, cyclized pentapeptides $(106,107)$ secreted by the necrotrophic fungus Cochliobolus victoriae that causes Victoria blight on susceptible oats (108). Fungal pathogenicity is solely attributed to the ability to produce victorin, as victorin deficient mutants are entirely non-pathogenic (109). While susceptibility of oats can be traced back to one dominant gene called Vb (110), it was later found that a single, dominant gene, called Locus Orchestrating Victorin Effects1 (LOV1), provides victorin susceptibility in Arabidopsis plants (111). Interestingly, only oat lines carrying Pc2, a resistance gene against crown rust, are susceptible to victorin producing C. victoriae isolates $(110,112)$. As studies to create plants resistant to both Victoria blight and crown rust were unsuccessful, it was suggested that Vb and Pc2 are the same gene conferring susceptibility and resistance respectively $(112,113)$. Further evidence for this hypothesis was provided by the discovery that victorin perception triggers a defence response in susceptible oats and Arabidopsis $(95,111)$ hinting that C. victoriae hijacks the classic gene-forgene interaction needed to provide resistance against crown rust and utilizes victorin to elicit host cell death via the same defense mechanism to suit its necrotrophic lifestyle.

The necrotrophic fungus Parastagonospora nodorum (formerly Stagonospora nodorum) is the causal agent of the Septoria nodorum blotch (SNB) disease on wheat $(114,115)$. Besides the ability to produce CWDEs and nonspecific toxins, P. nodorum has been characterized for its ability to produce a wide range of HSTs (also called necrotrophic effectors) that result in different levels of susceptibility depending on the wheat cultivar (116-119). So far, a total of nine interactions between necrotrophic effectors of $P$. nodorum and corresponding wheat susceptibility genes have been found (120-133). Furthermore, it was reported that homologs of the necrotrophic $P$. nodorum effector gene ToxA have been acquired via horizontal gene transfer and interspecific hybridization by the wheat pathogens P. tritici-repentis, Phaeosphaeria avenaria triti, and Bipolaris sorokiniana (134). So far, two host targets Snn1 and Tsn1, of P. nodorum necrotrophic effectors ToxA and Tox1, respectively, have been cloned $(135,136)$. While Tsn1 resembles a plant resistance gene structure as it harbors a serine/threonine protein kinase, a nucleotide binding, and leucinerich repeat domains (136), Snn1 is a wall associated kinase with a predicted transmembrane domain (135). However, in both cases interaction with a corresponding necrotrophic effector leads to a so called necrotrophic effector-triggered susceptibility $(124,135-137)$ in opposition to the conventional effector-trigger immunity (ETI) observed in most of the biotrophic interactions. Taken together, these studies suggest that some necrotrophic fungal pathogens use effectors to subvert the host resistance mechanism for their own benefit $(95,137,138)$.

Non-typical effectors: sRNAs

Small RNAs (SRNA) induce gene silencing by binding to Argonaute (AGO) proteins and directing the RNA-induced silencing complex (RISC) to genes with complementary sequences (139). As regulatory molecules, sRNAs are involved in a wide range of biological processes such as organ morphogenesis, genome modification, and adaptive responses to abiotic and 
biotic stresses (140-142). Both animals and plants have been reported to exchange sRNAs with parasites, pathogens, or symbiotic organisms in cross-kingdom sRNAs transfer (143). It is generally assumed that sRNAs from plants are integral components of plant responses to adverse environmental conditions, including host-microbial interactions $(142,144)$. While host sRNAs play important roles in pathogen resistance, pathogens also encode sRNAs to manipulate host defence responses and mediate virulence $(143,145,146)$. The necrotrophic fungus Botrytis cinerea infects almost all vegetable and fruit crops, causing major losses worldwide. Recently, it has been reported that some B. cinerea sRNAs (Bc-sRNAs) can silence Arabidopsis and tomato genes involved in immunity (145). The produced Bc-sRNAs hijack the host RNA interference (RNAi) machinery by binding to Arabidopsis AGO1. Furthermore, BcsRNAs silence host target immunity genes in both Arabidopsis and tomato plants during fungal infection (145). Cross-kingdom RNAi to suppress host immunity genes by hijacking host AGO1 has also been reported for $V$. dahliae (143). Arabidopsis ago1-27 mutants were less susceptible to the infection with $V$. dahliae than wild-type plants in both soil and root culture conditions (143). These results indicate that fungal pathogens and hosts utilize cross-kingdom RNAi to manipulate their interactions to their own benefit.

\section{Evolution of pathogen virulence}

As effectors are pathogen molecules that are crucial for establishing the parasitic symbiosis, hosts continuously evolve to intercept pathogen effectors or their activities with their immune receptor repertoire to halt pathogen ingress (9). To avoid or overcome such recognition, pathogens need to be able to swiftly purge or modify effectors that are intercepted by host immune systems, or evolve novel effectors to suppress the reinstated immune response, leading to an everlasting co-evolution between pathogen and host (7, 9). Based on genomics of plant pathogenic species, it has been proposed that many pathogens possess a bipartite genome architecture where effector genes cluster in repeat-rich dynamic compartments, a phenomenon that has been coined a "two-speed" genome $(147,148)$. These regions are typically repeat-rich, sometimes with active transposable elements (TEs), and often display increased structural polymorphism, increased point mutagenesis and positive selection (149-154). TEs are likely to contribute to pathogen adaptation by facilitating the swift evolution of effector catalogs by establishing genetic variability $(152,155)$, yet the underlying mechanisms remain largely unknown (156). However, genomic analysis in V. dahliae revealed active and passive contributions of TES, through transposable element activity, and through acting as substrate for homology-based double-strand repair pathways, respectively (152).

To control the spread and activity of TES, TE-rich genomic regions are often highly condensed in heterochromatin, which is directed by DNA methylation. As effector genes and other virulencerelated genes, such as toxin biosynthesis genes, often reside in TE-rich regions, TEs can impact the expression of these genes (157-159). Consequently, specific and differential methylation may be associated with adaptive evolution of two-speed pathogen genomes (156, 160, 161). Thus, TEs drive genome and transcriptome variability that, in turn, impacts pathogen adaptation (156). 


\section{Conclusion}

While all plant pathogenic fungi come across common plant defence mechanisms during host colonization, they employ different strategies to bypass these. As the on-going co-evolution with their hosts prompts pathogens to appropriately respond to modifications in host immunity in a timely manner, fungi need to continuously adapt their repertoire of virulence strategies to keep their parasitic relationships ongoing. A deep understanding of the molecular mechanisms underlying these virulence strategies and of host-pathogen interactions will result in the identification of precise virulence targets in the host plant. Such knowledge is paramount to improve current crop protection strategies or to design novel measures for disease control.

\section{Acknowledgements}

Work in the laboratory of B.P.H.J.T. is supported by the Research Council Earth and Life Sciences (ALW) of the Netherlands Organization of Scientific Research (NWO). The authors declare no conflict of interest.

\section{References}

1. Jiang Y, et al. (2017) Plants transfer lipids to sustain colonization by mutualistic mycorrhizal and parasitic fungi. Science 356(6343):1172-1175.

2. Rovenich H, Boshoven JC, \& Thomma BP (2014) Filamentous pathogen effector functions: Of pathogens, hosts and microbiomes. Current opinion in plant biology 20:96-103.

3. Mendgen K \& Hahn M (2002) Plant infection and the establishment of fungal biotrophy. Trends in plant science 7(8):352-356.

4. Whisson SC, et al. (2007) A translocation signal for delivery of oomycete effector proteins into host plant cells. Nature 450(7166):115-118.

5. Rafigi M, et al. (2010) Internalization of flax rust avirulence proteins into flax and tobacco cells can occur in the absence of the pathogen. Plant Cell 22(6):2017-2032.

6. Rafiqi M, Ellis JG, Ludowici VA, Hardham AR, \& Dodds PN (2012) Challenges and progress towards understanding the role of effectors in plant-fungal interactions. Current opinion in plant biology 15(4):477-482.

7. Jones JD \& Dangl JL (2006) The plant immune system. Nature 444(7117):323-329.

8. Thomma BPHJ, et al. (2001) Different micro-organisms differentially induce Arabidopsis disease response pathways. Plant Physiol Bioch 39(7-8):673-680.

9. Cook DE, Mesarich CH, \& Thomma BP (2015) Understanding plant immunity as a surveillance system to detect invasion. Annual Review of Phytopathology 53:541-563.

10. Felix G, Regenass M, \& Boller T (1993) Specific perception of subnanomolar concentrations of chitin fragments by tomato cells: Induction of extracellular alkalinization, changes in protein phosphorylation, and establishment of a refractory state. Plant J 4(2):307-316.

11. Shibuya N, Kaku H, Kuchitsu K, \& Maliarik MJ (1993) Identification of a novel high-affinity binding site for $\mathrm{N}$-acetylchitooligosaccharide elicitor in the membrane fraction from suspension-cultured rice cells. FEBS Lett 329(1-2):75-78.

12. Altenbach D \& Robatzek S (2007) Pattern recognition receptors: From the cell surface to intracellular dynamics. Molecular Plant-Microbe Interactions 20(9):1031-1039.

13. Boller T \& Felix G (2009) A renaissance of elicitors: Perception of microbe-associated molecular patterns and danger signals by pattern-recognition receptors. Annual review of plant biology 60:379-406.

14. Bolton MD (2009) Primary metabolism and plant defense-fuel for the fire. Molecular Plant-Microbe Interactions 22(5):487-497.

15. Macho AP \& Zipfel C (2014) Plant PRRs and the activation of innate immune signaling. Molecular cell 54(2):263272.

16. Ryder LS \& Talbot NJ (2015) Regulation of appressorium development in pathogenic fungi. Current opinion in plant biology 26:8-13.

17. Kleemann J, et al. (2012) Sequential delivery of host-induced virulence effectors by appressoria and intracellular hyphae of the phytopathogen Colletotrichum higginsianum. PLoS pathogens 8(4):e1002643.

18. Lombard V, Golaconda Ramulu H, Drula E, Coutinho PM, \& Henrissat B (2014) The carbohydrate-active enzymes database (CAZY) in 2013. Nucleic Acids Res 42(Database issue):490-4955. 
19. Zhao Z, Liu H, Wang C, \& Xu JR (2013) Comparative analysis of fungal genomes reveals different plant cell wall degrading capacity in fungi. BMC genomics 14:274.

20. Bolton MD, Thomma BP, \& Nelson BD (2006) Sclerotinia sclerotiorum (lib.) de Bary: Biology and molecular traits of a cosmopolitan pathogen. Molecular plant pathology 7(1):1-16.

21. Alghisi P \& Favaron F (1995) Pectin-degrading enzymes and plant-parasite interactions. European Journal of Plant Pathology 101(4):365-375.

22. Volpi C, et al. (2011) The ectopic expression of a pectin methyl esterase inhibitor increases pectin methyl esterification and limits fungal diseases in wheat. Molecular Plant-Microbe Interactions 24(9):1012-1019.

23. Tundo S, et al. (2016) Pyramiding PvPGIP2 and TAXI-III but not PVPGIP2 and PMEl enhances resistance against Fusarium graminearum. Molecular Plant-Microbe Interactions 29(8):629-639.

24. Paccanaro MC, et al. (2017) Synergistic effect of different plant cell wall-degrading enzymes is important for virulence of Fusarium graminearum. Molecular Plant-Microbe Interactions 30(11):886-895.

25. King R, et al. (2017) A conserved fungal glycosyltransferase facilitates pathogenesis of plants by enabling hyphal growth on solid surfaces. PLoS pathogens 13(10):e1006672.

26. Couto D \& Zipfel C (2016) Regulation of pattern recognition receptor signalling in plants. Nature reviews. Immunology 16(9):537-552.

27. Cote F, Roberts KA, \& Hahn MG (2000) Identification of high-affinity binding sites for the hepta- $\beta$-glucoside elicitor in membranes of the model legumes Medicago truncatula and Lotus japonicus. Planta 211(4):596-605.

28. Cosio EG, Feger M, Miller CJ, Antelo L, \& Ebel J (1996) High-affinity binding of fungal $\beta$-glucan elicitors to cell membranes of species of the plant family Fabaceae. Planta 200(1):92-99.

29. Sanchez-Vallet A, Mesters JR, \& Thomma BP (2015) The battle for chitin recognition in plant-microbe interactions. FEMS microbiology reviews 39(2):171-183.

30. Dean RA, et al. (2005) The genome sequence of the rice blast fungus Magnaporthe grisea. Nature 434(7036):980-986.

31. Fujikawa T, et al. (2012) Surface a-1,3-glucan facilitates fungal stealth infection by interfering with innate immunity in plants. PLoS pathogens 8(8):e1002882.

32. Oliveira-Garcia E \& Deising HB (2013) Infection structure-specific expression of $\beta$-1,3-glucan synthase is essential for pathogenicity of Colletotrichum graminicola and evasion of $\beta$-glucan-triggered immunity in maize. Plant Cell 25(6):2356-2378.

33. El Gueddari NE, Rauchhaus U, Moerschbacher BM, \& Deising HB (2002) Developmentally regulated conversion of surface-exposed chitin to chitosan in cell walls of plant pathogenic fungi. New Phytologist 156(1):103-112.

34. Oliveira-Garcia E \& Deising HB (2016) Attenuation of PAMP-triggered immunity in maize requires downregulation of the key $\beta-1,6$-glucan synthesis genes kre5 and kre6 in biotrophic hyphae of Colletotrichum graminicola. Plant J 87(4):355-375.

35. de Jonge R, et al. (2010) Conserved fungal LysM effector Ecp6 prevents chitin-triggered immunity in plants. Science 329(5994):953-955.

36. Sanchez-Vallet A, et al. (2013) Fungal effector Ecp6 outcompetes host immune receptor for chitin binding through intrachain LysM dimerization. eLife 2:e00790.

37. Buist G, Steen A, Kok J, \& Kuipers OP (2008) LysM, a widely distributed protein motif for binding to (peptido) glycans. Molecular microbiology 68(4):838-847.

38. van den Burg HA, Harrison SJ, Joosten MH, Vervoort J, \& de Wit PJ (2006) Cladosporium fulvum Avr4 protects fungal cell walls against hydrolysis by plant chitinases accumulating during infection. Molecular Plant-Microbe Interactions 19(12):1420-1430

39. van Esse HP, Bolton MD, Stergiopoulos I, de Wit PJ, \& Thomma BP (2007) The chitin-binding Cladosporium fulvum effector protein Avr4 is a virulence factor. Molecular Plant-Microbe Interactions 20(9):1092-1101.

40. Stergiopoulos I, et al. (2010) Tomato cf resistance proteins mediate recognition of cognate homologous effectors from fungi pathogenic on dicots and monocots. Proceedings of the National Academy of Sciences of the United States of America 107(16):7610-7615.

41. de Jonge R \& Thomma BP (2009) Fungal LysM effectors: Extinguishers of host immunity? Trends in microbiology 17(4):151-157.

42. Marshall R, et al. (2011) Analysis of two in planta expressed LysM effector homologs from the fungus mycosphaerella graminicola reveals novel functional properties and varying contributions to virulence on wheat. Plant Physiol 156(2):756-769.

43. Mentlak TA, et al. (2012) Effector-mediated suppression of chitin-triggered immunity by Magnaporthe oryzae is necessary for rice blast disease. Plant Cell 24(1):322-335.

44. Takahara H, et al. (2016) Colletotrichum higginsianum extracellular LysM proteins play dual roles in appressorial function and suppression of chitin-triggered plant immunity. New Phytologist 211(4):1323-1237.

45. Kombrink A, et al. (2017) Verticillium dahliae LysM effectors differentially contribute to virulence on plant hosts. Molecular plant pathology 18(4):596-608.

46. Rovenich H, Zuccaro A, \& Thomma BPHJ (2016) Convergent evolution of filamentous microbes towards evasion of glycan-triggered immunity. New Phytologist 212(4):896-901.

47. Fesel PH \& Zuccaro A (2016) B-glucan: Crucial component of the fungal cell wall and elusive mamp in plants. Fungal genetics and biology: FG \& B 90:53-60. 
48. Wawra S, et al. (2016) The fungal-specific $\beta$-glucan-binding lectin fgb1 alters cell-wall composition and suppresses glucan-triggered immunity in plants. Nature communications 7:13188.

49. Albersheim P \& Valent BS (1974) Host-pathogen interactions. Vii. Plant pathogens secrete proteins which inhibit enzymes of the host capable of attacking the pathogen. Plant Physiol 53(5):684-687.

50. Naumann TA, Wicklow DT, \& Kendra DF (2009) Maize seed chitinase is modified by a protein secreted by Bipolaris zeicola. Physiol Mol Plant P 74(2):134-141.

51. Naumann TA \& Wicklow DT (2010) Allozyme-specific modification of a maize seed chitinase by a protein secreted by the fungal pathogen stenocarpella maydis. Phytopathology 100(7):645-654.

52. Naumann TA, Wicklow DT, \& Price NP (2011) Identification of a chitinase-modifying protein from Fusarium verticillioides: Truncation of a host resistance protein by a fungalysin metalloprotease. The Journal of biological chemistry 286(41):35358-35366.

53. Jashni MK, et al. (2015) Synergistic action of a metalloprotease and a serine protease from Fusarium oxysporum f. Sp. Lycopersici cleaves chitin-binding tomato chitinases, reduces their antifungal activity, and enhances fungal virulence. Molecular Plant-Microbe Interactions 28(9):996-1008.

54. Bolwell GP, et al. (2002) The apoplastic oxidative burst in response to biotic stress in plants: A three-component system. Journal of Experimental Botany 53(372):1367-1376.

55. Sasaki K, et al. (2004) Ten rice peroxidases redundantly respond to multiple stresses including infection with rice blast fungus. Plant \& cell physiology 45(10):1442-1452.

56. Bindschedler LV, et al. (2006) Peroxidase-dependent apoplastic oxidative burst in Arabidopsis required for pathogen resistance. Plant J 47(6):851-863.

57. Lamb C \& Dixon RA (1997) The oxidative burst in plant disease resistance. Annual review of plant physiology and plant molecular biology 48:251-275.

58. Orozco-Cardenas ML, Narvaez-Vasquez J, \& Ryan CA (2001) Hydrogen peroxide acts as a second messenger for the induction of defense genes in tomato plants in response to wounding, systemin, and methyl jasmonate. Plant Cell 13(1):179-191.

59. Qi J, Wang J, Gong Z, \& Zhou JM (2017) Apoplastic ROS signaling in plant immunity. Current opinion in plant biology 38:92-100.

60. Levine A, Tenhaken R, Dixon R, \& Lamb C (1994) $\mathrm{H}_{2} \mathrm{O}_{2}$ from the oxidative burst orchestrates the plant hypersensitive disease resistance response. Cell 79(4):583-593.

61. Wu GS, et al. (1997) Activation of host defense mechanisms by elevated production of $\mathrm{h}_{2} \mathrm{O}_{2}$ in transgenic plants. Plant Physiol 115(2):427-435.

62. Doehlemann G, et al. (2009) Pep1, a secreted effector protein of Ustilago maydis, is required for successful invasion of plant cells. PLoS pathogens 5(2):e1000290.

63. Hemetsberger C, Herrberger C, Zechmann B, Hillmer M, \& Doehlemann G (2012) The Ustilago maydis effector pep1 suppresses plant immunity by inhibition of host peroxidase activity. PLoS pathogens 8(5):e1002684.

64. Prusky D \& Yakoby N (2003) Pathogenic fungi: Leading or led by ambient ph? Molecular plant pathology 4(6):509-516.

65. Prusky D, McEvoy JL, Leverentz B, \& Conway WS (2001) Local modulation of host ph by Colletotrichum species as a mechanism to increase virulence. Molecular Plant-Microbe Interactions 14(9):1105-1113.

66. Masachis S, et al. (2016) A fungal pathogen secretes plant alkalinizing peptides to increase infection. Nature microbiology 1(6):16043.

67. Murphy E \& De Smet I (2014) Understanding the ralf family: A tale of many species. Trends in plant science 19(10):664-671.

68. Thynne E, et al. (2017) Fungal phytopathogens encode functional homologues of plant rapid alkalinization factor (ralf) peptides. Molecular plant pathology 18(6):811-824.

69. van der Hoorn RA (2008) Plant proteases: From phenotypes to molecular mechanisms. Annual review of plant biology 59:191-223.

70. Jashni MK, Mehrabi R, Collemare J, Mesarich CH, \& de Wit PJ (2015) The battle in the apoplast: Further insights into the roles of proteases and their inhibitors in plant-pathogen interactions. Front Plant Sci 6:584.

71. van Esse HP, et al. (2008) The Cladosporium fulvum virulence protein avr2 inhibits host proteases required for basal defense. Plant Cell 20(7):1948-1963.

72. Shabab M, et al. (2008) Fungal effector protein avr2 targets diversifying defense-related cys proteases of tomato. Plant Cell 20(4):1169-1183.

73. Kruger J, et al. (2002) A tomato cysteine protease required for cf-2-dependent disease resistance and suppression of autonecrosis. Science 296(5568):744-747.

74. Rooney HC, et al. (2005) Cladosporium avr2 inhibits tomato rcr3 protease required for cf-2-dependent disease resistance. Science 308(5729):1783-1786.

75. Doehlemann G, Reissmann S, Assmann D, Fleckenstein M, \& Kahmann R (2011) Two linked genes encoding a secreted effector and a membrane protein are essential for Ustilago maydis-induced tumour formation. Molecular microbiology 81(3):751-766.

76. Mueller AN, Ziemann S, Treitschke S, Assmann D, \& Doehlemann G (2013) Compatibility in the Ustilago maydismaize interaction requires inhibition of host cysteine proteases by the fungal effector pit2. PLoS pathogens 9(2):e1003177. 
77. Glazebrook J (2005) Contrasting mechanisms of defense against biotrophic and necrotrophic pathogens. Annual Review of Phytopathology 43:205-227.

78. Thomma BPHJ, et al. (1998) Separate jasmonate-dependent and salicylate-dependent defense-response pathways in Arabidopsis are essential for resistance to distinct microbial pathogens. Proc Natl Acad Sci USA 95(25):15107-15111.

79. Djamei A, et al. (2011) Metabolic priming by a secreted fungal effector. Nature 478(7369):395-398.

80. Rabe F, Ajami-Rashidi Z, Doehlemann G, Kahmann R, \& Djamei A (2013) Degradation of the plant defence hormone salicylic acid by the biotrophic fungus Ustilago maydis. Mol Microbiol 89(1):179-188.

81. Liu T, et al. (2014) Unconventionally secreted effectors of two filamentous pathogens target plant salicylate biosynthesis. Nature communications 5:4686.

82. Kloppholz S, Kuhn H, \& Requena N (2011) A secreted fungal effector of glomus intraradices promotes symbiotic biotrophy. Current biology : CB 21(14):1204-1209

83. Plett JM, et al. (2014) Effector missp7 of the mutualistic fungus laccaria bicolor stabilizes the populus jaz6 protein and represses jasmonic acid (ja) responsive genes. Proceedings of the National Academy of Sciences of the United States of America 111(22):8299-8304

84. Chanclud E \& Morel JB (2016) Plant hormones: A fungal point of view. Molecular plant pathology 17(8):12891297.

85. Chanclud E, et al. (2016) Cytokinin production by the rice blast fungus is a pivotal requirement for full virulence. PLoS pathogens 12(2):e1005457.

86. Yin C, Park JJ, Gang DR, \& Hulbert SH (2014) Characterization of a tryptophan 2-monooxygenase gene from puccinia graminis f. Sp. Tritici involved in auxin biosynthesis and rust pathogenicity. Molecular Plant-Microbe Interactions 27(3):227-235.

87. Reineke G, et al. (2008) Indole-3-acetic acid (iaa) biosynthesis in the smut fungus Ustilago maydis and its relevance for increased iaa levels in infected tissue and host tumour formation. Molecular plant pathology $9(3): 339-355$

88. Sorensen JL, et al. (2017) The cereal pathogen Fusarium pseudograminearum produces a new class of active cytokinins during infection. Molecular plant pathology.

89. Stergiopoulos I \& de Wit PJ (2009) Fungal effector proteins. Annual Review of Phytopathology 47:233-263.

90. Kale SD \& Tyler BM (2011) Entry of oomycete and fungal effectors into plant and animal host cells. Cellular microbiology 13(12):1839-1848.

91. Ribot C, et al. (2013) The Magnaporthe oryzae effector avr1-co39 is translocated into rice cells independently of a fungal-derived machinery. Plant J 74(1):1-12.

92. Petre B \& Kamoun S (2014) How do filamentous pathogens deliver effector proteins into plant cells? PLoS biology 12(2):e1001801.

93. Giraldo MC, et al. (2013) Two distinct secretion systems facilitate tissue invasion by the rice blast fungus Magnaporthe oryzae. Nature communications 4:1996

94. Khang CH, et al. (2010) Translocation of Magnaporthe oryzae effectors into rice cells and their subsequent cellto-cell movement. Plant Cell 22(4):1388-1403.

95. Wolpert TJ, Dunkle LD, \& Ciuffetti LM (2002) Host-selective toxins and avirulence determinants: What's in a name? Annual Review of Phytopathology 40:251-285.

96. Daub ME \& Ehrenshaft M (2000) The photoactivated Cercospora toxin cercosporin: Contributions to plant disease and fundamental biology. Annual Review of Phytopathology 38:461-490

97. Birben E, Sahiner UM, Sackesen C, Erzurum S, \& Kalayci O (2012) Oxidative stress and antioxidant defense. The World Allergy Organization journal 5(1):9-19.

98. Blokhina O, Virolainen E, \& Fagerstedt KV (2003) Antioxidants, oxidative damage and oxygen deprivation stress: A review. Annals of botany $91 \mathrm{Spec} \mathrm{No}(2): 179-194$.

99. Weltmeier F, et al. (2011) Transcript profiles in sugar beet genotypes uncover timing and strength of defense reactions to Cercospora beticola infection. Molecular Plant-Microbe Interactions 24(7):758-772.

100. de Jonge R, et al. (2018) Gene cluster conservation provides insight into cercosporin biosynthesis and extends production to the genus Colletotrichum. Proceedings of the National Academy of Sciences, doi:10.1073/ pnas.1712798115.

101. Tsuge T, et al. (2013) Host-selective toxins produced by the plant pathogenic fungus Alternaria alternata. FEMS microbiology reviews 37(1):44-66.

102. Levings lii CS, Rhoads DM, \& Siedow JN (1995) Molecular interactions of bipolaris maydis T-toxin and maize. Canadian Journal of Botany 73(S1):483-489.

103. Yang G, Turgeon BG, \& Yoder OC (1994) Toxin-deficient mutants from a toxin-sensitive transformant of cochliobolus heterostrophus. Genetics 137(3):751-757.

104. Yun SH, Turgeon BG, \& Yoder OC (1998) Remi-induced mutants of mycosphaerella zeae-maydis lacking the polyketide PM-toxin are deficient in pathogenesis to corn. Physiol Mol Plant P 52(1):53-66.

105. Meena M, et al. (2017) Alternaria toxins: Potential virulence factors and genes related to pathogenesis. Frontiers in microbiology 8:1451.

106. Wolpert TJ, Macko V, Acklin W, Jaun B, \& Arigoni D (1986) Structure of minor host-selective toxins from Cochliobolus-victoriae. Experientia 42(11-12):1296-1299. 
107. Wolpert TJ, et al. (1985) Structure of victorin-C, the major host-selective toxin from Cochliobolus-victoriae. Experientia 41(12):1524-1529.

108. Meehan F \& Murphy H (1947) Differential phytotoxicity of metabolic by-products of Helminthosporium victoriae. Science 106(2751):270-271.

109. Scheffer RP, Nelson RR, \& Ullstrup AJ (1967) Inheritance of toxin production and pathogenicity in Cochliobolus carbonum and Cochliobolus victoriae. Phytopathology 57(12):1288-8.

110. Welsh JN, Peturson B, \& Machacek JE (1954) Associated inheritance of reaction to races of crown rust, Puccinia-coronata-avenae ERIKSs, and to victoria blight, Helminthosporium-victoriae M and M in oats. Can J Bot 32(1):55-8

111. Gilbert BM \& Wolpert TJ (2013) Characterization of the Lov1-mediated, victorin-induced, cell-death response with virus-induced gene silencing. Mol Plant Microbe In 26(8):903-917.

112. Litzenberger SC (1948) Inheritance of resistance to specific races of crown rust, stem rust, and to helminthosporium blight of oats.

113. Mayama S, Bordin APA, Morikawa T, Tanpo H, \& Kato H (1995) Association of avenalumin accumulation with co-segregation of victorin sensitivity and crown rust resistance in oat lines carrying the Pc-2 gene. Physiol Mol Plant P 46(4):263-274.

114. King JE, Cook RJ, \& Melville SC (1983) A review of septoria diseases of wheat and barley. Annals of Applied Biology 103(2):345-373.

115. Oliver RP, Friesen TL, Faris JD, \& Solomon PS (2012) Stagonospora nodorum: From pathology to genomics and host resistance. Annual Review of Phytopathology 50:23-43.

116. Friesen TL \& Faris JD (2010) Characterization of the wheat-Stagonospora nodorum disease system: What is the molecular basis of this quantitative necrotrophic disease interaction? Can J Plant Pathol 32(1):20-28.

117. Wicki W, Messmer M, Winzeler M, Stamp P, \& Schmid JE (1999) In vitro screening for resistance against Septoria nodorum blotch in wheat. TAG. Theoretical and applied genetics. Theoretische und angewandte Genetik 99(78):1273-1280.

118. Wicki W, Winzeler M, Schmid JE, Stamp P, \& Messmer M (1999) Inheritance of resistance to leaf and glume blotch caused by Septoria nodorum berk. in winter wheat. TAG. Theoretical and applied genetics. Theoretische und angewandte Genetik 99(7-8):1265-1272.

119. Keller B, Winzeler H, Winzeler M, \& Fried PM (1994) Differential sensitivity of wheat embryos against extracts containing toxins of Septoria nodorum: First steps towards in vitro selection. J Phytopathol 141(3):233-240.

120. Liu ZH, et al. (2004) Genetic and physical mapping of a gene conditioning sensitivity in wheat to a partially purified host-selective toxin produced by Stagonospora nodorum. Phytopathology 94(10):1056-1060.

121. Liu ZH, et al. (2004) Quantitative trait loci analysis and mapping of seedling resistance to Stagonospora nodorum leaf blotch in wheat. Phytopathology 94(10):1061-1067.

122. Liu Z, et al. (2006) The Tsn1-Toxa interaction in the wheat-Stagonospora nodorum pathosystem parallels that of the wheat-tan spot system. Genome 49(10):1265-1273.

123. Friesen TL, et al. (2006) Emergence of a new disease as a result of interspecific virulence gene transfer. Nature genetics 38(8):953-956.

124. Friesen TL, Meinhardt SW, \& Faris JD (2007) The Stagonospora nodorum-wheat pathosystem involves multiple proteinaceous host-selective toxins and corresponding host sensitivity genes that interact in an inverse genefor-gene manner. Plant J 51(4):681-692.

125. Friesen TL, Zhang Z, Solomon PS, Oliver RP, \& Faris JD (2008) Characterization of the interaction of a novel Stagonospora nodorum host-selective toxin with a wheat susceptibility gene. Plant Physiol 146(2):682-693.

126. Friesen TL, et al. (2009) Host-selective toxins produced by Stagonospora nodorum confer disease susceptibility in adult wheat plants under field conditions. Theoretical and Applied Genetics 118(8):1489-1497.

127. Friesen Tl, Chu C, Xu SS, \& Faris JD (2012) Sntox5-Snn5: A novel Stagonospora nodorum effector-wheat gene interaction and its relationship with the Sntoxa-Tsn1 and Sntox3-Snn3-b1 interactions. Molecular Plant Pathology 13(9):1101-1109.

128. Abeysekara NS, Friesen TL, Keller B, \& Faris JD (2009) Identification and characterization of a novel hosttoxin interaction in the wheat-Stagonospora nodorum pathosystem. TAG. Theoretical and applied genetics. Theoretische und angewandte Genetik 120(1):117-126.

129. Abeysekara NS, Faris JD, Chao S, McClean PE, \& Friesen TL (2012) Whole-genome QTL analysis of Stagonospora nodorum blotch resistance and validation of the SnTox4-Snn4 interaction in hexaploid wheat. Phytopathology 102(1):94-104.

130. Chu C-G, Faris JD, Xu SS, \& Friesen TL (2010) Genetic analysis of disease susceptibility contributed by the compatible Tsn1-Sntoxa and Snn1-Sntox1 interactions in the wheat-Stagonospora nodorum pathosystem. Theoretical and Applied Genetics 120(7):1451-1459

131. Zhang Z, et al. (2011) Two putatively homoeologous wheat genes mediate recognition of Sntox3 to confer effector-triggered susceptibility to Stagonospora nodorum. The Plant Journal 65(1):27-38.

132. Shi G, et al. (2015) The wheat Snn7 gene confers susceptibility on recognition of the Parastagonospora nodorum necrotrophic effector sntox7. The Plant Genome 8(2).

133. Gao Y, et al. (2015) Identification and characterization of the Sntox6-Snn6 interaction in the Parastagonospora nodorum-wheat pathosystem. Molecular Plant-Microbe Interactions 28(5):615-625. 
134. McDonald MC, Ahren D, Simpfendorfer S, Milgate A, \& Solomon PS (2017) The discovery of the virulence gene ToxA in the wheat and barley pathogen bipolaris sorokiniana. Molecular plant pathology:DOI: 10.1111/mpp.12535.

135. Shi G, et al. (2016) The hijacking of a receptor kinase-driven pathway by a wheat fungal pathogen leads to disease. Science Advances 2(10).

136. Faris JD, et al. (2010) A unique wheat disease resistance-like gene governs effector-triggered susceptibility to necrotrophic pathogens. Proceedings of the National Academy of Sciences of the United States of America 107(30):13544-13549.

137. Liu Z, et al. (2012) The cysteine rich necrotrophic effector SnTox1 produced by Stagonospora nodorum triggers susceptibility of wheat lines harboring Snn1. PLoS pathogens 8(1):e1002467.

138. Liu Z, et al. (2015) Necrotrophic effector-triggered susceptibility (NETS) underlies the barley-Pyrenophora teres f. teres interaction specific to chromosome 6H. Molecular plant pathology 16(2):188-200.

139. Castel SE \& Martienssen RA (2013) RNA interference in the nucleus: Roles for small RNAs in transcription, epigenetics and beyond. Nature reviews. Genetics 14(2):100-112.

140. Carrington JC \& Ambros V (2003) Role of microRNAs in plant and animal development. Science 301(5631):336-338.

141. Ruiz-Ferrer $\vee$ \& Voinnet $O$ (2009) Roles of plant small RNAs in biotic stress responses. Annual review of plant biology 60:485-510.

142. Katiyar-Agarwal S \& Jin H (2010) Role of small RNAs in host-microbe interactions. Annual Review of Phytopathology 48:225-246.

143. Wang M, et al. (2016) Bidirectional cross-kingdom RNAi and fungal uptake of external RNAs confer plant protection. Nature plants 2:16151.

144. Zhang X, et al. (2011) Arabidopsis argonaute 2 regulates innate immunity via miRNA393(*)-mediated silencing of a golgi-localized SNARE gene, memb12. Molecular cell 42(3):356-366.

145. Weiberg A, et al. (2013) Fungal small RNAs suppress plant immunity by hijacking host RNA interference pathways. Science 342(6154):118-123.

146. Chaloner T, van Kan JA, \& Grant-Downton RT (2016) RNA 'information warfare' in pathogenic and mutualistic interactions. Trends in plant science 21(9):738-748.

147. Croll D \& McDonald BA (2012) The accessory genome as a cradle for adaptive evolution in pathogens. PLoS pathogens 8(4).

148. Raffaele S \& Kamoun S (2012) Genome evolution in filamentous plant pathogens: Why bigger can be better. Nature Reviews Microbiology 10(6):417-430.

149. Raffaele S, et al. (2010) Genome evolution following host jumps in the Irish potato famine pathogen lineage. Science 330(6010):1540-1543.

150. Rouxel T, et al. (2011) Effector diversification within compartments of the Leptosphaeria maculans genome affected by repeat-induced point mutations. Nature Commun 2.

151. de Jonge R, et al. (2013) Extensive chromosomal reshuffling drives evolution of virulence in an asexual pathogen. Genome Res 23(8):1271-1282.

152. Faino L, et al. (2016) Transposons passively and actively contribute to evolution of the two-speed genome of a fungal pathogen. Genome Res 26(8):1091-1100.

153. Möller M \& Stukenbrock EH (2017) Evolution and genome architecture in fungal plant pathogens. Nature reviews. Microbiology 15:756-771.

154. Dong YH, et al. (2015) Global genome and transcriptome analyses of Magnaporthe oryzae epidemic isolate 9806 uncover novel effectors and pathogenicity-related genes, revealing gene gain and lose dynamics in genome evolution. PLoS pathogens 11(4).

155. Bao J, et al. (2017) PacBio sequencing reveals transposable elements as a key contributor to genomic plasticity and virulence variation in Magnaporthe oryzae. Molecular Plant 10(11):1465-1468.

156. Seidl MF \& Thomma BPHJ (2017) Transposable elements direct the coevolution between plants and microbes. Trends Genet 33(11):842-851.

157. Soyer JL, et al. (2014) Epigenetic control of effector gene expression in the plant pathogenic fungus Leptosphaeria maculans. Plos Genet 10(3):e1004227.

158. Connolly LR, Smith KM, \& Freitag M (2013) The Fusarium graminearum histone H3 K27 methyltransferase KMT6 regulates development and expression of secondary metabolite gene clusters. Plos Genet 9(10).

159. Chujo T \& Scott B (2014) Histone H3K9 and H3K27 methylation regulates fungal alkaloid biosynthesis in a fungal endophyte-plant symbiosis. Mol Microbiol 92(2):413-434.

160. Chen K, et al. (2017) Methyltransferase SETD2-mediated methylation of STAT1 is critical for interferon antiviral activity. Cell 170(3):492-+.

161. Seidl MF, Cook DE, \& Thomma BPHJ (2016) Chromatin biology impacts adaptive evolution of filamentous plant pathogens. PLoS pathogens 12(11). 



\section{Homologs of Verticillium dahliae effector Ave1 contribute to virulence of fungal pathogens of diverse plant hosts}




\section{Abstract}

Verticillium dahliae is a soil-borne fungal pathogen with a wide host range including many crops. Tomato immune receptor Ve1 confers resistance to $\mathrm{V}$. dahliae race 1 strains that express VdAve1. On plants that do not carry Ve1, Ave1 acts as a virulence factor of $V$. dahliae. Homologs of Ave1 are mostly found in plants and in a handful of fungal plant pathogens including Fusarium oxysporum (FoAve1), Cercospora beticola (CbAve1), and Colletotrichum higginsianum (ChAve1). In the bacterium Xanthomonas axonopodis the Ave1 homolog XacPNP was previously described as a virulence factor. The homologs FoAve1, CbAve1 are recognized by Ve1 although not as efficient as VdAve1. In this study, we analyzed the role of the individual Ave1 homologs in virulence. We generated targeted gene deletions in Fusarium oxysporum, Cercospora beticola and Colletotrichum higginsianum and showed that both FoAve1 and CbAve1 are virulence factors in contrast to ChAve1 that does not contribute to the virulence of the pathogen. We subsequently tested whether Ave1 homologs FoAve1, CbAve1, ChAve1 and XacPNP act in a similar fashion as VdAve1 by expressing these genes in a V. dahliae VdAve1 deletion strain. We concluded that VdAve1 on the one hand and FoAve1, CbAve1, ChAve1 and XacPNP on the other hand act in different manners. 


\section{Introduction}

Plants are surrounded by microbes including oomycetes, fungi, bacteria and viruses. To prevent colonization by harmful microbes, plants evolved pattern recognition receptors (PRRs) that recognize microbe associated molecular patterns (MAMPs) and activate MAMP-triggered immunity (MTI) (1, 2). MAMPs generally are structural components such as bacterial elongation factor Tu (EF-Tu), flagellin, lipopolysaccharides (LPS), fungal chitin and oomycete $\beta$-glucans, which are typically conserved across genera of microbes. Successful pathogens secrete effector proteins that modulate host immunity to overcome PRR-mediated plant defence (3, 4). Such effectors are often lineage-specific, facilitate colonization of the plant, and therefore contribute to virulence of the pathogen. In turn, plants evolved immune receptors, typically called resistance (R) proteins, which recognize particular effectors or their activities to mount an immune response known as effector-triggered immunity (ETI) (5). To overcome recognition by the plant, pathogens have to mutate or loose the recognized effector or, alternatively, evolve new effectors to suppress the ETI response. Nevertheless, not all pathogen-secreted molecules follow the strict MAMP-effector dichotomy but rather display traits of either class of molecules, and therefore MTI and ETI responses cannot strictly be separated (6). Typical examples of such pathogen-secreted molecules are Nep1-like proteins, which are virulence factors that are dispersed throughout three kingdoms of life that act as a MAMP at least in Arabidopsis $(7,8)$. Although less widely distributed, phenomena have been observed for homologs of the Avr4 effector from the tomato leaf mold fungus Cladosporium fulvum (9), and for homologs of the Ave1 effector from the vascular wilt fungus Verticillium dahliae (4). This has inspired the proposal of the so-called invasion model, which states that plants evolved receptors for all types of molecules, pathogen- as well as host-derived, that can betray microbial invasion to mount the appropriate immune responses (10).

V. dahliae is a soil-borne fungal pathogen that causes vascular wilt in over 200 plant species, including important crop species (11). Resistance to $V$. dahliae is mediated by the immune receptor Ve1 $(12,13)$, of which homologs were found in mint, hop and cotton amongst other plant species (14-16). Recently, the effector protein that is secreted by $V$. dahliae race 1 strains and that activates Ve1-mediated resistance was identified as Ave1, and all resistance-breaking race 2 strains analyzed thus far lack the complete Ave1 gene $(4,17)$. Importantly, Ave1 deletion strains show reduced aggressiveness on tomato plants that lack $V e 1$, revealing that Ave1 acts as a virulence factor on this host species. Additionally, Ave1 was also shown to act as a virulence factor of $V$. dahliae on Arabidopsis plants (4). However, how Ave1 contributes to V. dahliae virulence on these plant hosts remains unknown thus far.

Intriguingly, although pathogen effectors are typically lineage-specific traits, many homologs of Ave1 can be found in public databases (4). Remarkably, a wealth of Ave1 homologs was found in plants as well as in a handful of fungal plant pathogens, including Fusarium oxysporum (FoAve1), Cercospora beticola (CbAve1) and Colletotrichum higginsianum (ChAve1), and in the bacterial plant pathogen Xanthomonas axonopodis (XacPNP) $(4,18)$. Interestingly, it was shown that Ve1 is not confined to $V$. dahliae Ave1 (VdAve1), as Ve1 is able to also recognize FoAve1 and CbAve1, while ChAve1 is not recognized (4). Consequently, Ve1 was indeed found to be able to recognize 
F. oxysporum in tomato and mount a defense response (4). Remarkably, Ve1 was furthermore found to recognize an endogenous Ave1 homolog (SIAve1) that shares a high degree of identity with VdAve1 (4).

Most plant homologs of Ave1 have been annotated either as plant natriuretic peptides (PNPs) or as expansin-like proteins, and functionally analyzed members were implicated in the regulation of water and ion homeostasis, and consequently in many downstream processes including growth, net water uptake, photosynthesis, stomatal opening and gas exchange (19-22). The observation that the microbial-derived Ave1 homologs do not follow the phylogeny of the species in which they occur is generally taken as evidence for horizontal acquisition, and it has thus been speculated that the microbial Ave1 homologs were acquired from plants $(4,18)$. In this respect it is interesting that the $X$. axonopodis homolog XacPNP affects homeostasis and photosynthesis in citrus plants, and thus promotes bacterial proliferation as a virulence factor in citrus plants $(18,23,24)$. In this study, we investigate whether the Ave1 homologs of several fungal pathogens act as genuine virulence factors.

\section{Results}

\section{Characterization of Ve1-mediated tomato defence against Fusarium oxysporum}

We previously demonstrated that tomato Ve1 can recognize FoAve1, leading to a defense response that affects infection of Ve1-carrying tomato by F. oxysporum (4). Because recognition of FoAve1 by Ve1-carrying tomato plants does not lead to full immunity against $F$. oxysporum we investigated the occurrence of expression of FoAve1 in two F. oxysporum strains, Bt.01 and Fol4287, in tomato plants lacking Ve1 with reverse-transcription PCR at 14 DPI. Clear expression of FoAve1 was monitored at 14 DPI (Figure 1A).

To provide further evidence for the role of FoAve1-recognition in Ve1-mediated tomato defense against Fusarium wilt, we generated FoAve1 deletion mutants in F. oxysporum strains Bt.01 and Fol4287 through homologous recombination. Deletion of FoAve1 was confirmed by PCR (Suppl. Figure $1 \mathrm{~A}$ ) and the deletion strains were subsequently evaluated for their ability to colonize Ve1 tomato plants. As anticipated, inoculation of Ve1 tomato plants with FoAve1 deletion strains resulted in more extensive symptoms of Fusarium wilt disease when compared to tomato plants inoculated with the corresponding wild type strains (Figure $1 \mathrm{~B}$ and $1 \mathrm{C}$ ). Altogether, these data show that tomato Ve1 is able to recognize FoAve1 and activate a defense response that inhibits colonization by F. oxysporum, albeit that the response does not fully arrest the fungus and immunity is not fully established. At later stages after inoculation clear symptoms of wilt disease are observed on Ve1 plants.

\section{FoAve1 is a virulence factor of $F$. oxysporum during tomato colonization}

According to the paradigm that plant immune receptors recognize crucial virulence factors of microbial pathogens, it is expected that FoAve1 contributes to F. oxysporum virulence. To test this hypothesis, FoAve1 deletion strains were inoculated on tomato plants lacking Ve1. Indeed, 
A

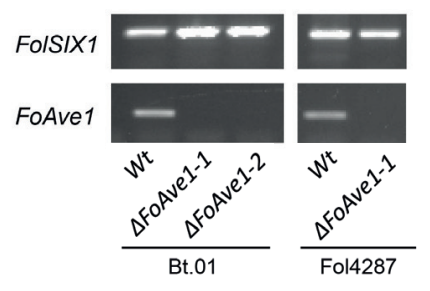

B

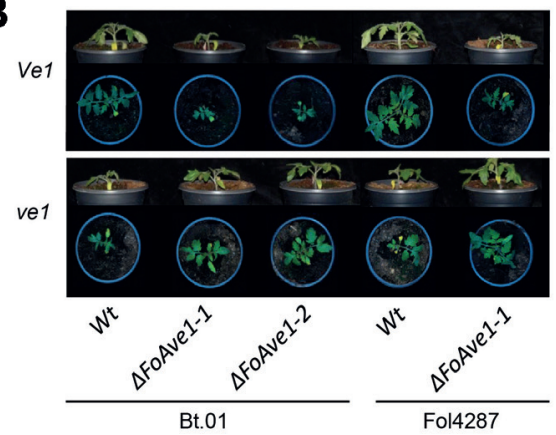

C
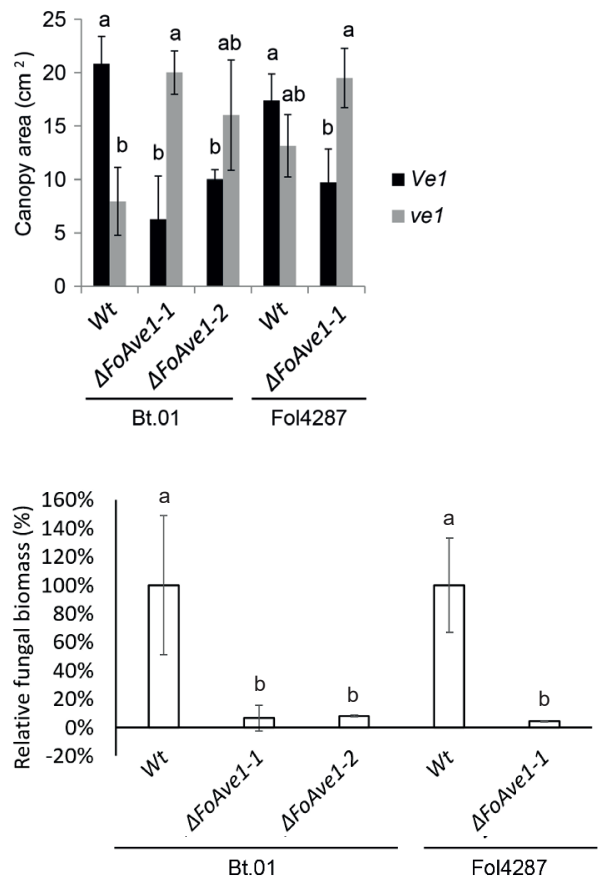

Figure 1. FoAve1 is a virulence factor recognized by tomato Ve1. (A) Expression of FoAve1 in F. oxysporum in wild type (Wt) strains Bt.01 and Fol4287 and the FoAve1 deletion strains ( $\triangle F o A v e 1$ ) inoculated on tomato at $14 \mathrm{DPI}$. (B) (Upper) FoAve1 deletion strains ( $\triangle F o A v e 1$ ) of $F$. oxysporum strains Bt.01 and Fol4287 escape recognition by tomato Ve1 compared with the corresponding wild type (Wt) strains evidenced by stunted Ve1 plants at $14 \mathrm{DPI}$. (Lower) FoAve1 deletion strains display reduced virulence compared with the corresponding F. oxysporum wild type strains Bt.01 and Fol4287 on tomato lacking Ve1 (ve1) evidenced by reduced stunting at 14 DPI. (C) Canopy area of Ve1 tomato and tomato lacking Ve1 after inoculation with FoAve1 deletion strains and the corresponding F. oxysporum wild type strains at 14 DPI. (D) FoAve1 deletion strains display reduced virulence compared to the corresponding F. oxysporum wild type strains Bt.01 and Fol4287 on tomato lacking Ve1 evidenced by reduced fungal biomass at 14 DPI. 8 plants were pooled per 2 plants ( $n=4)$. Different letter labels indicate statistically significant differences $(P<0.05)$. Similar results were observed in at least 3 biological replications for all experiments. 
FoAve1 deletion strains displayed reduced virulence on tomato plants when compared to the corresponding F. oxysporum wild type strain as visualized by the difference in canopy area of the inoculated tomato plants (Figure 1B and 1C). In addition, plants inoculated with FoAve1 deletion strains resulted in less fungal colonization compared to those inoculated with wild type F. oxysporum (Figure 1D). These results suggest that FoAve1 is a virulence factor of F. oxysporum, albeit that its contribution to virulence appears to be relatively minor.

\section{ChAve1 is not a virulence factor of $C$. higginsianum during Arabidopsis colonization}

Besides the vascular wilt fungi $V$. dahliae and F. oxysporum, that belong to the class of Sordariomycetes, an Ave1 homolog is found in the foliar Sordariomycete C. higginsianum (ChAve1). Using real-time PCR, ChAve1 expression was monitored in wild type C. higginsianum during colonization of Arabidopsis plants from 1 to $4 \mathrm{DPI}$, by which time the plant tissue was completely macerated. No expression of ChAve1 was detected at any of these time points. Nevertheless, we investigated whether ChAve1 contributes to virulence in this foliar pathogen. For this purpose, ChAve1 deletion strains were generated that, after confirmation with PCR (Suppl. Figure 1B), were evaluated for aggressiveness on Arabidopis thaliana. As expected ChAve1 deletion strains did not show reduced virulence when compared to the corresponding wild type C. higginsianum, as infections resulted in lesions with similar sizes (Figure 2A). Moreover, similar levels of pathogen biomass were recorded in plants inoculated with the wild-type fungus and the deletion strains (Figure 2B). Together these results show that ChAve1 is not a virulence factor of C. higginsianum on Arabidopsis, likely due to the lack of ChAve1 expression.
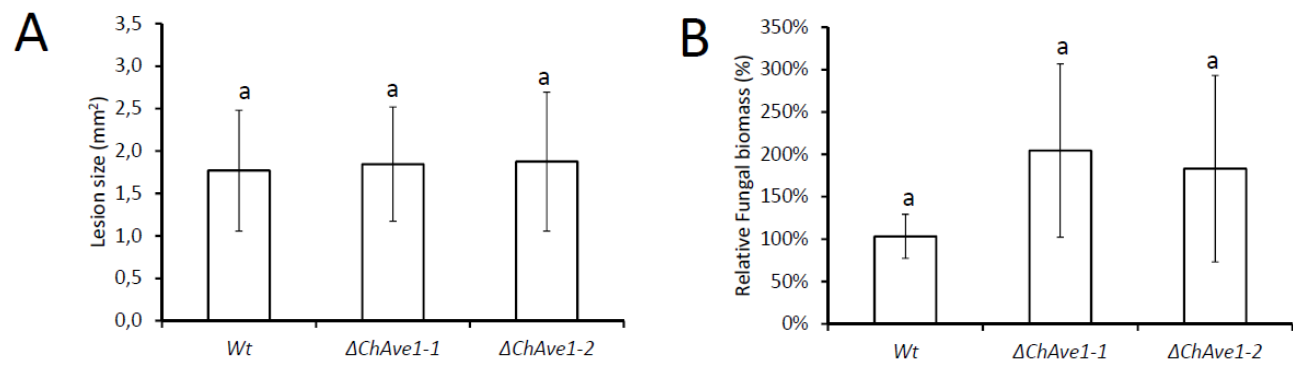

Figure 2. ChAve1 is not a virulence factor of $\boldsymbol{C}$. higginsianum. (A) ChAve1 deletion strains ( $\triangle \mathrm{ChAve} 1$ ) show a similar lesion size compared to the corresponding C. higginsianum wild type strain (Wt) at 3 DPI on 3-week-old A. thaliana. Twenty four lesions on three A. thaliana plants were measured per strain. No significant difference was observed $(P<0.05)$. (B) No difference in fungal biomass was observed at $4 \mathrm{DPI}$ on spray inoculated 3-weekold Arabidopsis plants $(n=4)$. Different letter labels indicate statistically significant differences $(P<0.05)$. Similar results were observed in at least 3 biological replications for all experiments in this figure.

\section{CbAve1 is a virulence factor of $C$. beticola during sugar beet colonization}

Besides Sordariomycetes, Ave1 homologs were also identified as we previously identified an Ave1 homolog in the Dothidiomycete C. beticola (CbAve1). Expression of CbAve1 was investigated in C. beticola during colonization of sugar beet plants between 3 and 18 DPI using real-time PCR. Clear expression was detected between these time points. To evaluate the contribution 
A

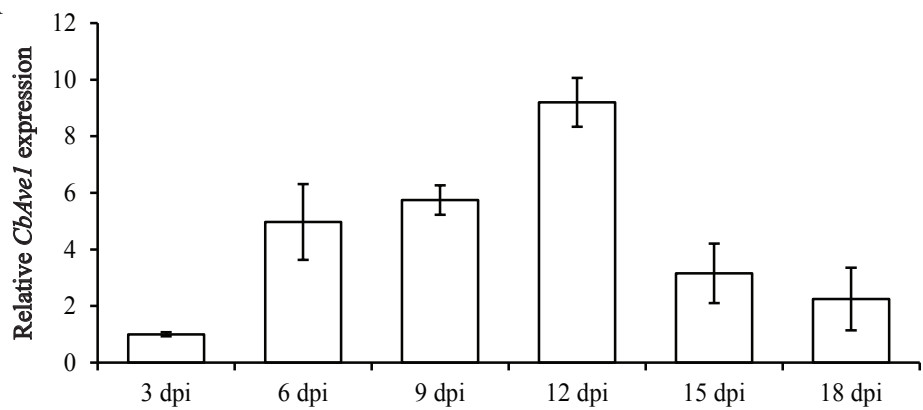

B

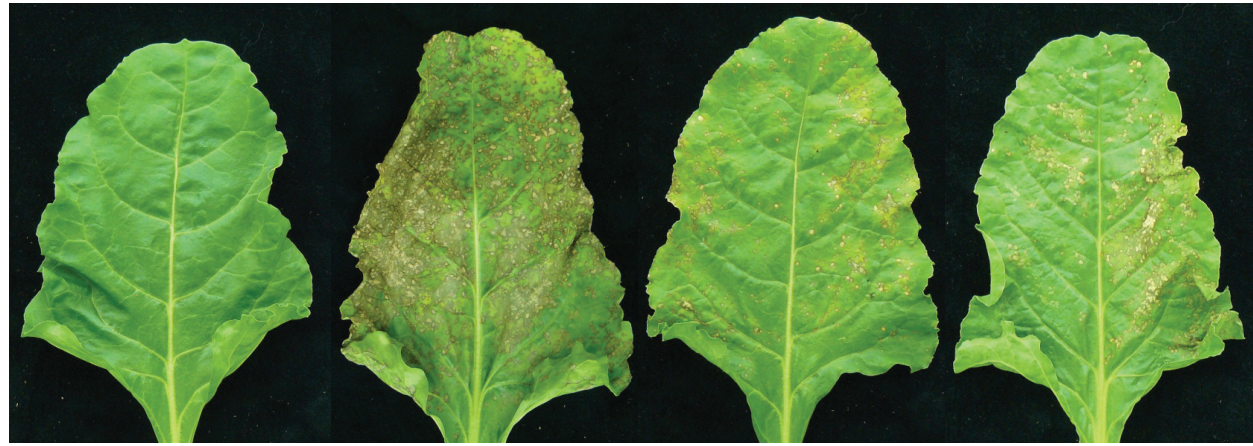

Mock

WT

$\triangle$ CbAvel \#1

$\triangle$ CbAvel \#2

Cercospora beticola

C

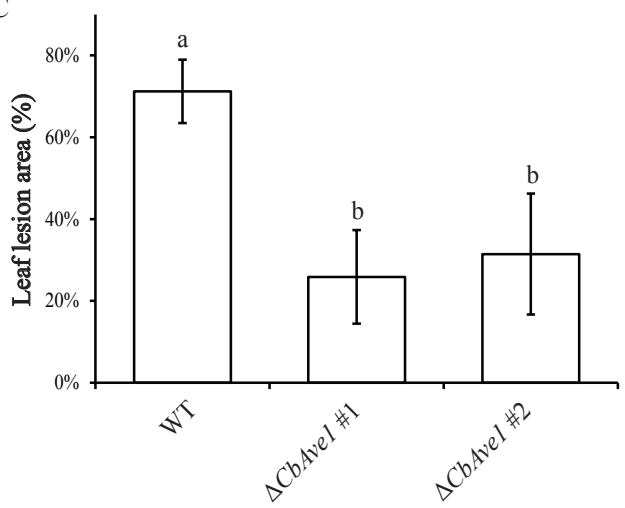

D

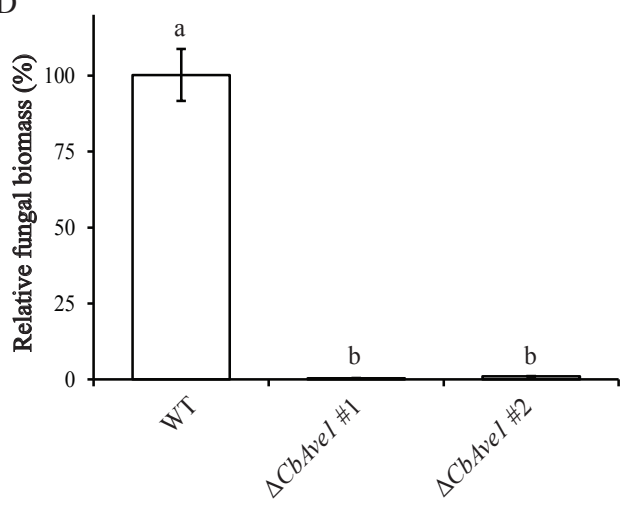

Figure 3. CbAve1 is a virulence factor of $C$. beticola. (A) Expression of $C b A v e 1$ during infection of $C$. beticola on sugar beet. Leaves of 6 - to 7-week-old sugar beet plants were inoculated with wild-type $C$. beticola and collected at regular intervals from 3 to 18 days post inoculation (DPI). RT-qPCR was performed to determine the relative expression levels of CbAve1 using the $C$. beticola actin gene as a reference, and compared with CbAve1 expression in C. beticola in sugar beet plants upon the wild-type $C$. beticola inoculation at 3 DPI, which is set to 1. (B) CbAve1 deletion strains $(\triangle C b A v e 1 \# 1$ and $\triangle C b A v e 1 \# 2$ ) show reduced virulence compared to the corresponding $C$. beticola wild type strain (WT) visualized by a reduction in lesion area on sugar beet leaves at 15 DPI. (C) Quantification of leaf lesion caused by $C$. beticola on sugar beet plants at 15 DPI $(n>5)$. Bars represent the average percentage of leaf lesion area of whole leaf area with standard deviations. (D) Fungal biomass determined with qPCR in Cercospora-inoculated sugar beet plants at $15 \mathrm{DPI}$. The fungal biomass in sugar beet plants upon inoculation with the wild-type $C$. beticola is set to 100 $\%$ (control). Different letter labels indicate statistically significant differences $(P<0.05)$. 
of CbAve1 in C. beticola virulence, CbAve1 deletion strains were generated and confirmed with PCR. For this pathogen, deletion of the Ave1 homolog resulted in reduced virulence compared to the corresponding wild type strain, as infection resulted in reduced numbers and size of the lesions (Figure 3A; B). As expected, CbAve1 deletion strains showed reduced colonization on sugar beet plants compared to the corresponding C. beticola wild type strain (Figure 3C). Taken together, these results show that CbAve1 is a virulence factor of $C$. beticola.

\section{Functional diversification among plant and fungal Ave1 homologs}

VdAve1 is a virulence factor of $V$. dahliae that shows a high degree of identity with homologs from plants. It has therefore been proposed that $V d A v e 1$ has been acquired by $V$. dahliae from plants through horizontal gene transfer (4). However, the function of VdAve1 through which it contributes to $V$. dahliae aggressiveness remains presently enigmatic. Similarly, the function of the Ave1 homologs that are found in various pathogens also remains unclear. In order to evaluate whether all homologs share their functionality, we tested whether they can complement the virulence defect that is observed upon VdAve1 deletion in V. dahliae. To this end, we transformed one of the VdAve1 deletion strains of $V$. dahliae with constructs to drive expression of the plant homologs derived from tomato (S. lycopersicum; SIAve1) and grape (V. vinifera; VVAve1) by the $V$. dahliae VdAve1 promotor (4). To confirm that the plant homologs were expressed in $V$. dahliae upon colonization of tomato we monitored SIAve1 and VVAve1 expression with PCR. At 14 DPI we monitored clear expression of SIAve1 and VVAve1 in 3 transformants for each construct (Suppl. Figure 2a). Subsequently, we evaluated the ability of these transformants to cause disease on tomato plants lacking Ve1. All VdAve1 deletion strains carrying SIAve1 and VVAve1 showed a similar phenotype and fungal colonization as the corresponding VdAve1 deletion strain (Figure 4; Suppl. Figure 2). These results suggest that two plant Ave1 homologs that share a high identity with VdAve1 are unable to restore virulence in a VdAve1 deletion strain, suggesting that Ave1 homologs from plants act in a different manner as VdAve1.

Subsequently, we evaluated whether microbial Ave1 homologs can reinstall the compromised virulence of $V$. dahliae that results from VdAve1 deletion. To this end, we transformed one of the VdAve1 deletion strains with constructs to drive expression of the Ave1 homologs derived from F. oxysporum (FoAve1), C. higginsianum (ChAve1) and C. beticola (CbAve1) by the VdAve1 promoter. We also included the Ave1 homolog derived from the plant pathogenic bacterium Xanthomonas axonopodis (XacPNP) that was previously described as a virulence factor (18). We confirmed in a minimum of 2 transformants that the constructs were expressed (Suppl. Figure 2a). Subsequently, we tested the $V$. dahliae transformants on tomato lacking Vel and compared them with the corresponding wild type $V$. dahliae and the $V$. dahliae VdAve1 deletion strain complemented with VdAve1. VdAve1 deletion strains expressing FoAve1, CbAve1, ChAve1 and XacPNP showed a similar disease phenotype and fungal colonization as VdAve1 deletion strains (Figure 4; Suppl. Figure 2). (Figure 4; Suppl. Figure 2). These results show that the Ave1 homologs FoAve1, CbAve1, ChAve1 and XacPNP cannot restore virulence in a VdAve1 deletion strain which may suggest that Ave1 homologs of $V$. dahliae on the one hand, and F. oxysporum, C. beticola, C. higginsianum and $X$. axonopodis on the other hand, contribute to fungal virulence in a different manner. 

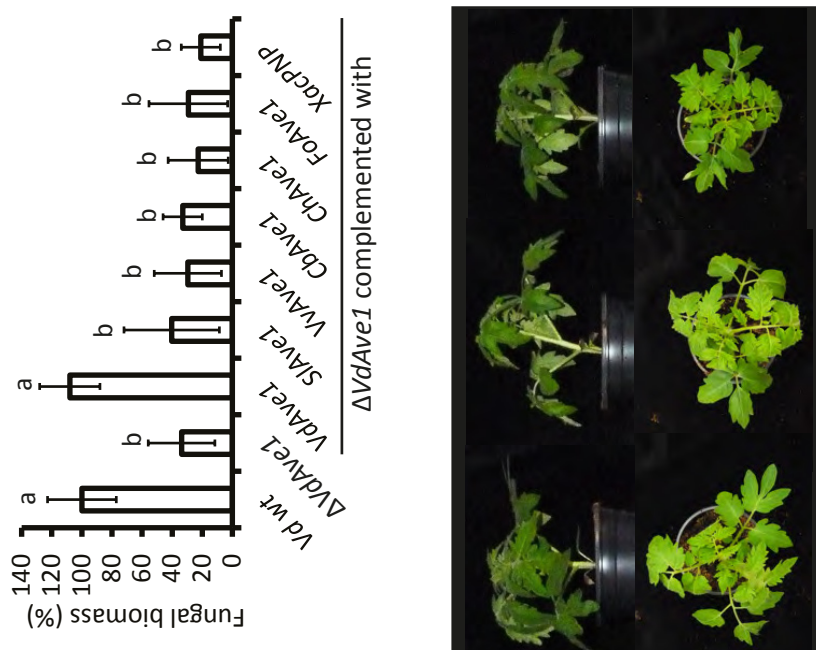

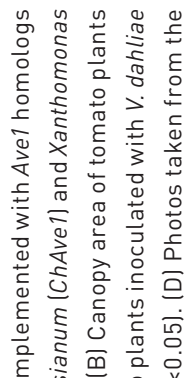

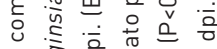

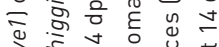

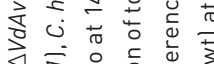

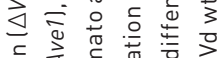

웍웡ㅇㅇㅇㅇㅇㅇ

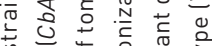

$\cup \quad(\%)$ ssemo!̣ |esunf
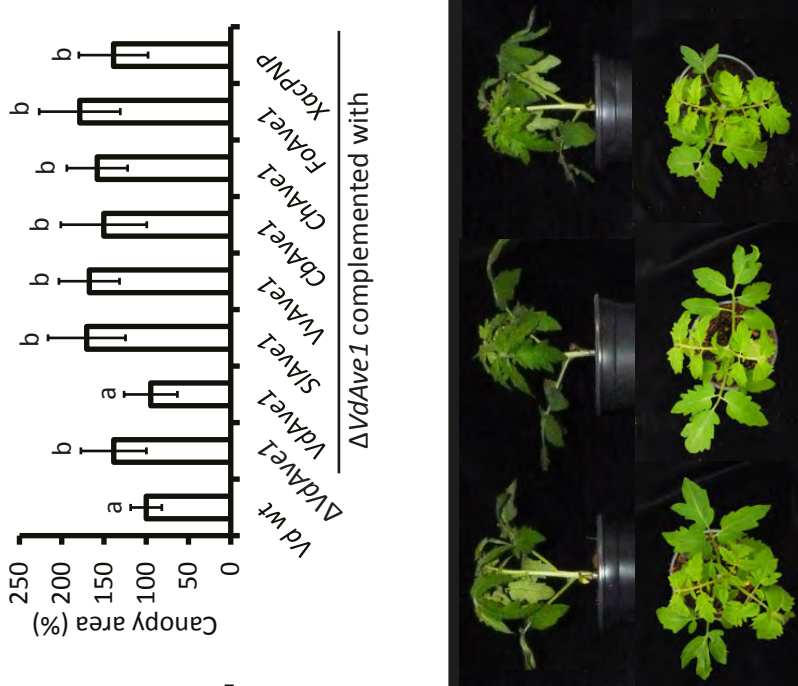

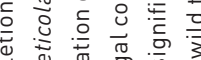

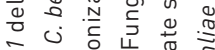

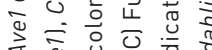

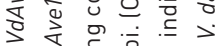

离

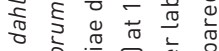

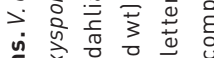

ตั $\vec{x} \div \geq$

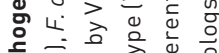

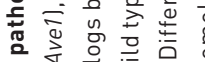

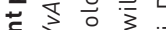

范落

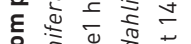

난

过

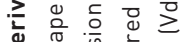

ஃ

जू ज्ञ है

ص

(\%) еәле Kdoueว

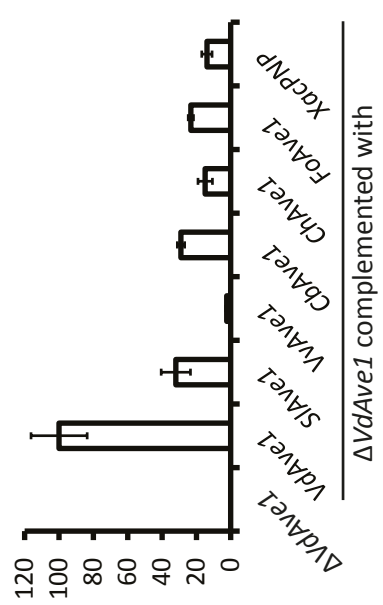

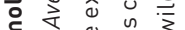

है ज

হ ह

ये

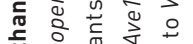

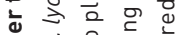

는

ह

ป

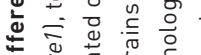

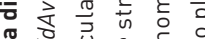

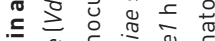

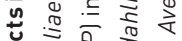

क $\frac{\pi}{2} \frac{1}{2}$ o

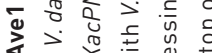

造

$>$ 눙 웧

ป

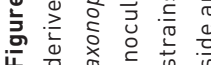

(\%) uo!ssə. dx $\exists$

○

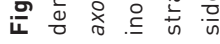




\section{Discussion}

\section{The role of FoAve1 in recognition by tomato immune receptor Ve1}

We have previously shown that recognition of FoAve1 by Ve1 leads to a defense response (4) and that Ve1 tomato plants can recognize F. oxysporum. To confirm that FoAve1 is involved in recognition we first analyzed FoAve1 expression. A previous study could not detect FoAve1 expression in F. oxysporum after inoculation on tomato (25). However, in the conditions that we tested clear FoAve1 expression was monitored in F. oxysporum on tomato at 14 DPI. In addition, deletion of FoAve1 resulted in abolishment of recognition by Ve1. Our data suggest that the incomplete disease resistance cannot be attributed to a lack of FoAve1 expression, but may be the result of a less efficient detection of this Ave1 homolog by Ve1. Similar differences in recognition efficiencies were previously observed for Cladosporium fulvum effector Ecp2 and its homolog MfEcp2 of Mycosphaerella fijiensis when coexpressed with tomato immune receptor Cf-Ecp2 (9).

\section{Some Ave1 homologs act as virulence factors}

Previously, we have shown that Ave1 is a virulence factor of $V$. dahliae (4). In this study, we tested wheter Ave1 homologs in other plant pathogens act as virulence factors as well. We showed that deletion of Ave1 homologs in F. oxysporum and C. beticola resulted in reduced aggressiveness on their respective plant hosts. In contrast, the Ave1 homolog ChAve1 of the foliar pathogen C. higginsianum does not contribute to virulence due to the lack of expression in planta. Possibly, the lack of expression is due to the availability of effectors with a similar function that render ChAve1 functionality redundant. Alternatively, recognition of ChAve1 by a host immune receptor posed pressure on C. higginsianum to avoid recognition, resulting in the lack of ChAve1 expression. Interestingly, homologs of tomato Ve1 have been identified in many other plant species, of which some within as well as outside the Solanaceae family have been shown to be functional immune receptors (26).

\section{Functional diversification among Ave1 homologs}

Since Ave1 homologs derived from plants display high similarity to Ave1, it has been suggested that Ave1 was acquired through horizontal gene transfer from plants (4). As the function of the Ave1 homologs remain unknown we tested whether the Ave1 homologs share the same functionality by complementing a $V$. dahiae Ave1 deletion strain with homologs derived from plants and various plant pathogens. Highly similar Ave1 homologs derived from plants are unable to restore virulence in an Ave1 deletion strain. Therefore we conclude that Ave1 homologs derived from plants act in a different manner as Ave1. Similarly, Ave1 homologs derived from F. oxysporum, C. beticola, C. higginsianum and $X$. axonopodis cannot restore the virulence penalty caused by the deletion of Ave1. Therefore, we conclude that Ave1 from V. dahliae on the one hand, and Ave1 homologs derived from F. oxysporum, C. beticola, C. higginsianum and X. axonopodis on the other hand, contribute to virulence in a different manner. Similarly, functional diversification within effector families has been observed for LysM and NLP effectors $(27,28)$. Alternatively, it is important to note that V. dahliae, C. beticola, C. higginsianum and $X$. axonopodis have diverse plant hosts and that the reason for the inability to restore virulence in an $V$. dahliae Ave1 deletion strain could be that each homolog targets a host-specific protein. This would for example explain why the homolog of $C$. beticola that likely 
targets a sugar beet protein cannot restore virulence in an V. dahliae Ave1 deletion strain colonizing tomato. Host specific effector adaptation has been suggested for the closely related oomycetes Phytophtora infestans and P. mirabilis that are pathogens on different hosts. Homologous effectors of both pathogens act more efficiently on their specific host target than on a "foreign" host target (29). Nevertheless, the finding that the Ave1 homolog from F. oxysporum f.sp. lycopersici cannot complement the VdAve1 deletion strain for virulence on tomato argues against this hypothesis.

\section{Materials and Methods}

\section{Deletion strains}

Deletion strains were generated in F. oxysporum and C. higginsianum by amplifying 1,5 kb sequences (Suppl. Table 1) flanking the coding sequence of FoAve1 and ChAve1, respectively, and cloned as previously described (30) into vector pRF-HU2 containing a nourseothricin cassette for selection. For the complementation of V. dahliae Ave1 deletion strains FoAve1, CbAve1, ChAve1, SIPNP, VVPNP and XacPNP were obtained by gene synthesis (Eurofins Genomics, Ebersberg, Germany). The synthesized genes were then cloned into vector pFBT005 under the VdAve1 promoter, containing a nourseothricin cassette for selection. F. oxysporum (Fol4287 and Bt.01) and C. higginsianum (IMI349063A) conidiospores were transformed on a Hybond-N+ filter by $A$. tumefaciens carrying the pRF-HU2 plasmid containing the constructs and a V. dahliae Ave1 deletion strain (JR2) was transformed by A. tumefaciens carrying the pFBT0 05 plasmid containing the constructs as previously described (31). Transformants were then selected on potato dextrose agar (PDA) (Thermo Fisher Scientific Inc, Breda, The Netherlands) containing hygromycin B (Duchefa Biochemie BV, Haarlem, The Netherlands) for selection of F. oxysporum, C. beticola and C. higginsianum transformants or nourseothricin sulphate (Sigma Aldrich Chemie BV, Zwijndrecht, The Netherlands) for selection of $V$. dahliae transformants. After five to seven days at room temperature, individual transformants were transferred from the filter to fresh PDA plates with the appropriate selection and incubated for ten days (31). To verify the transformants, fungal spores were collected and genomic DNA was extracted followed by a PCR to test the presence of the hygromycin or nourseothricin cassette and the presence of the construct in the genome (Suppl. Table 1).

CbAve1 deletion mutants of $C$. beticola were generated using the split-marker approach described by Catlett, et al. (32). Genomic DNA of the wild type C. beticola strain 09-40 and pDAN vector (33) served as PCR templates to generate split-marker PCR constructs used for transformation. Primers are listed in Suppl. Table 1. PEG-mediated transformation of the wild type C. beticola strain 09-40 was performed as previously described (34). Site-directed gene replacement was confirmed by the absence of PCR product using split-marker CbAve1 $1 \mathrm{~F}$ forward primer of the $5^{\prime}$ flank of the target gene and MDB-760 reverse primer designed on the coding sequence of CbAve1.

\section{Pathogen inoculations}

Plants were grown in soil in the greenhouse at 210C/19oC during $16-\mathrm{h} / 8$-h day/night periods, respectively, with $70 \%$ relative humidity and $100 \mathrm{~W}$ m-2 supplemental light when the intensity dropped below $150 \mathrm{~W}$ m-2. 
For V. dahliae and F. oxysporum inoculations, 10-day-old tomato (Solanum lycopersicum; cv. MoneyMaker or MoneyMaker 35S:Ve1) (13) were uprooted, rinsed in water and dipped for 5 minutes in a suspension of 106 conidiospores per $\mathrm{mL}$ of water harvested from 1- to 2-week-old $V$. dahliae or F. oxysporum cultures on PDA as previously described (13). Control plants received the same treatment, but the roots were dipped in water without conidiospores. After replanting in fresh soil, plants were incubated at standard greenhouse conditions. Disease development was monitored up to 21 days post inoculation (DPI).

For C. higginsianum inoculations 3-week-old Arabidopsis (A. thaliana ecotype Co-0 or Col-0 35S:Ve1) (35) were inoculated on the leaves with either $2 \mu \mathrm{l}$ drops or sprayed with a suspension of 106 conidia per $\mathrm{mL}$ of water harvested from 1- to 2-week-old C. higginsianum cultures on Mathurs' agar as previously described $(36,37)$. Control plants received the same treatment, but the leaves were sprayed with water without conidiospores. After sealing the plants inside a transparent closed box lined with wet tissue paper to provide high humidity, they were incubated at $25^{\circ} \mathrm{C}$, under a 16 -h/ 8-h light/dark regime (36). Disease development was monitored up to 4 DPI.

For C. beticola inoculations 6- to 7-week-old sugar beet plants (Beta vulgaris) were inoculated by evenly spaying spore suspension of 105 conidia per $\mathrm{mL}$ of water on the lower side of the leaves harvested from C. beticola cultures growing on V8 solid medium as previously described (38). Control plants received the same treatment, but the leaves were sprayed with water. Plants were incubated at standard greenhouse conditions and disease development was monitored up to $18 \mathrm{DPI}$.

\section{Fungal biomass and gene expression}

Stem sections of tomato, cut from the base of the stem up to the cotyledons, were collected at 7 and 14 DPI from plant inoculated with F. oxysporum or V. dahliae. Arabidopsis leaves were collected at 1-4 DPI after inoculation with C. higginsianum. Sugar beet leaves were collected at regular intervals between 3 and 18 DPI from plants inoculated with C. beticola. Collected plant tissue was flash frozen in liquid nitrogen and ground to powder, of which an aliquot of 100 mg was used for RNA extraction with the Quick-RNATM Miniprep kit (Zymo Research Europe GmbH, Freiburg, Germany), and CDNA was synthesized using M-MLV Reverse Transcriptase (Promega Benelux BV, Leiden, The Netherlands). Fungal biomass was determined by quantifying the expression of VAGAPDH, FOTUB, ChTUB and CbACt relative to the expression of SIRUB (tomato), AtRub (Arabidopsis), BvAct (sugar beet), respectively. Expression of VdAve1, FoAve1, CbAve1, ChAve1, SIPNP and VVPNP was determined by quantifying the expression relative to the expression of VdGAPDH, FOTUB, ChTUB and CbAct, respectively (Suppl. Table 1). Realtime PCR was carried out on an ABI7300 PCR machine (Applied Biosystems, Life Technologies Europe BV, Bleiswijk, The Netherlands) in combination with the qPCR SensiMix kit (BioLine, GC Biotech BV, Alphen aan den Rijn, The Netherlands). The following Real-time PCR conditions were used: an initial $95^{\circ} \mathrm{C}$ denaturation step for 10 minutes followed by denaturation for 15 seconds at $95^{\circ} \mathrm{C}$, annealing for 60 seconds at $60^{\circ} \mathrm{C}$, and extension at $72^{\circ} \mathrm{C}$ for 40 cycles. 


\section{Achknowledgements}

Work in the laboratory of BPHJT is supported by the Research Council Earth and Life Sciences (ALW) of the Netherlands Organization for Scientific Research (NWO). We thank Bert Essenstam and Pauline Sanderson of Unifarm for excellent plant care. We thank Jelle Postma, Dorien Oppelaar and Saskia Bosman for technical support.

\section{References}

1. Boller $T$ \& Felix G (2009) A renaissance of elicitors: Perception of microbe-associated molecular patterns and danger signals by pattern-recognition receptors. Annu Rev Plant Biol 60:379-406.

2. Dodds PN \& Rathjen JP (2010) Plant immunity: Towards an integrated view of plant-pathogen interactions. Nat Rev Genet 11(8):539-548.

3. Rovenich H, Boshoven JC, \& Thomma BPHJ (2014) Filamentous pathogen effector functions: Of pathogens, hosts and microbiomes. Curr Opin Plant Biol 20:96-103.

4. de Jonge R, et al. (2012) Tomato immune receptor Ve1 recognizes effector of multiple fungal pathogens uncovered by genome and RNA sequencing. Proc Natl Acad Sci U S A 109(13):5110-5115.

5. Jones JD \& Dangl JL (2006) The plant immune system. Nature 444(7117):323-329.

6. Thomma BPHJ, Nurnberger T, \& Joosten MHAJ (2011) Of PAMPs and effectors: The blurred PTI-ETI dichotomy. Plant Cell 23(1):4-15.

7. Böhm H, et al. (2014) A conserved peptide pattern from a widespread microbial virulence factor triggers pattern-induced immunity in Arabidopsis. PLoS Pathog 10(11):e1004491.

8. Oome S, et al. (2014) Nep1-like proteins from three kingdoms of life act as a microbe-associated molecular pattern in Arabidopsis. Proc Natl Acad Sci U S A 111(47):16955-16960.

9. Stergiopoulos I, et al. (2010) Tomato Cf resistance proteins mediate recognition of cognate homologous effectors from fungi pathogenic on dicots and monocots. Proc Natl Acad Sci U S A 107(16):7610-7615.

10. Cook DE, Mesarich CH, \& Thomma BPHJ (2015) Understanding plant immunity as a surveillance system to detect invasion. Annu Rev Phytopathol.

11. Fradin EF \& Thomma BPHJ (2006) Physiology and molecular aspects of Verticillium wilt diseases caused by $V$. dahliae and V. albo-atrum. Mol Plant Pathol 7(2):71-86.

12. Kawchuk LM, et al. (2001) Tomato Ve disease resistance genes encode cell surface-like receptors. Proceedings of the National Academy of Sciences of the United States of America 98(11):6511-6515.

13. Fradin EF, et al. (2009) Genetic dissection of Verticillium wilt resistance mediated by tomato Ve1. Plant physiology 150(1):320-332.

14. Vining K \& Davis T (2009) Isolation of a Ve homolog, $m V e 1$, and its relationship to verticillium wilt resistance in Mentha longifolia (I.) huds. Mol Genet Genomics 282(2):173-184.

15. Zhang $Y$, et al. (2011) Cloning and characterization of a verticillium wilt resistance gene from Gossypium barbadense and functional analysis in Arabidopsis thaliana. Plant Cell Rep 30(11):2085-2096.

16. Majer A, Javornik B, Cerenak A, \& Jakse J (2014) Development of novel EST-derived resistance gene markers in hop (Humulus lupulus l.). Mol Breed 33(1):61-74.

17. Faino L, et al. (2016) Transposons passively and actively contribute to evolution of the two-speed genome of a fungal pathogen. Genome Res 26(8):1091-1100

18. Gottig N, et al. (2008) Xanthomonas axonopodis pv. citri uses a plant natriuretic peptide-like protein to modify host homeostasis. Proc Natl Acad Sci U S A 105(47):18631-18636.

19. Gehring C \& Irving H (2003) Natriuretic peptides - a class of heterologous molecules in plants. Int J Biochem Cell Biol 35(9):1318-1322.

20. Pharmawati M, Maryani MM, Nikolakopoulos T, Gehring CA, \& Irving HR (2001) Cyclic gmp modulates stomatal opening induced by natriuretic peptides and immunoreactive analogues. Plant Physiol Biochem 39(5):385-394.

21. Maryani M, Bradley G, Cahill D, \& Gehring C (2001) Natriuretic peptides and immunoreactants modify osmoticumdependent volume changes in Solanum tuberosum l. mesophyll cell protoplasts. Plant Sci 161(3):443-452.

22. Ludidi N, et al. (2004) A recombinant plant natriuretic peptide causes rapid and spatially differentiated K+, NA+ and $\mathrm{H}+$ flux changes in Arabidopsis thaliana roots. Plant Cell Physiol 45(8):1093-1098.

23. Garavaglia BS, et al. (2010) A eukaryotic-acquired gene by a biotrophic phytopathogen allows prolonged survival on the host by counteracting the shut-down of plant photosynthesis. PLOS One 5(1):e8950

24. Nembaware V, Seoighe C, Sayed M, \& Gehring C (2004) A plant natriuretic peptide-like gene in the bacterial pathogen Xanthomonas axonopodis may induce hyper-hydration in the plant host: A hypothesis of molecular mimicry. BMC Evol Biol 4(1):10.

25. Schmidt SM, et al. (2013) Mites in the promoters of effector genes allow prediction of novel virulence genes in Fusarium oxysporum. BMC Genomics 14(1):119. 
26. Song Y, et al. (2017a) Broad taxonomic characterization of verticillium wilt resistance genes reveals ancient origin of the tomato Ve1 immune receptor. Mol Plant Pathol 18:195-209.

27. Kombrink A \& Thomma BPHJ (2013) LysM effectors: Secreted proteins supporting fungal life. PLOS Pathogens 9(12):e1003769.

28. Santhanam P, et al. (2012) Evidence for functional diversification within a fungal NEP1-like protein family. Molecular Plant-Microbe Interactions 26(3):278-286.

29. Dong S, et al. (2014) Effector specialization in a lineage of the Irish potato famine pathogen. Science 343(6170):552-555.

30. Frandsen RJ, Andersson JA, Kristensen MB, \& Giese H (2008) Efficient four fragment cloning for the construction of vectors for targeted gene replacement in filamentous fungi. BMC Mol Biol 9(1):70.

31. Santhanam $P$ (2012) Random insertional mutagenesis in fungal genomes to identify virulence factors. Plant fungal pathogens. Methods in molecular biology, ed Bolton MD TB (Humana press, Totowa), Vol 835, pp 509-517.

32. Catlett NL, Lee B-N, Yoder O, \& Turgeon BG (2003) Split-marker recombination for efficient targeted deletion of fungal genes. Fungal Genetics Reports 50(1):9-11.

33. Friesen $T L$, et al. (2006) Emergence of a new disease as a result of interspecific virulence gene transfer. Nature Genetics 38:953.

34. Bolton MD, et al. (2016) RNA-sequencing of Cercospora beticola DMI-sensitive and -resistant isolates after treatment with tetraconazole identifies common and contrasting pathway induction. Fungal Genetics and Biology 92:1-13.

35. Fradin EF, et al. (2011) Interfamily transfer of tomato Ve1 mediates Verticillium resistance in Arabidopsis. Plant Physiol 156(4):2255-2265.

36. O'Connell R, et al. (2004) A novel Arabidopsis-Colletotrichum pathosystem for the molecular dissection of plant-fungal interactions. Mol. Plant Microbe Interact. 17(3):272-282.

37. Sherriff C, et al. (1994) Ribosomal DNA sequence analysis reveals new species groupings in the genus Colletotrichum. Exp Mycol 18(2):121-138.

38. Bolton MD, Birla K, Rivera-Varas V, Rudolph KD, \& Secor GA (2012) Characterization of Cbcyp51 from field isolates of. Phytopathology 102(3):298-305. 


\section{Supplementary data}

Suppl. Table 1. Primers used in this study

\begin{tabular}{|c|c|c|}
\hline Primer & Sequence $\left(5^{\prime}-3^{\prime}\right)$ & Notes \\
\hline SIRub_QPCR_F & GAACAGTTTCTCACTGTTGAC & S. lycopersicum Rubisco \\
\hline SIRub_QPCR_R & CGTGAGAACCATAAGTCACC & S. lycopersicum Rubisco \\
\hline FolsIX1_F & GTCTCACGAGCCAAGTCTACC & F. oxysporum Six1 \\
\hline FolsIX1_R & GAACCGCAGCCTCTTGAGCAT & F. oxysporum Six1 \\
\hline FolTub_F & CTCTGGCAACAAGTATGTTCCC & F. oxysporum Tubulin \\
\hline FolTub_R & TTGTCGGGACGGAAGAGCTGA & F. oxysporum Tubulin \\
\hline FolAve1_QPCR_F3 & ATATCGGAACTGCAAATATTCTCAAC & F. oxysporum Ave1 \\
\hline FolAve1_QPCR_R3 & CTTATACATTTCATCGTATACAGTCTGC & F. oxysporum Ave1 \\
\hline AtRub_QPCR_F & GCAAGTGTTGGGTTCAAAGCTGGTG & A. thaliana Rubisco \\
\hline AtRub_QPCR_R & CCAGGTTGAGGAGTTACTCGGAATGCTG & A. thaliana Rubisco \\
\hline ChELF1a_F & CTGGTACAAGGGTTGGGAGA & C. higginsianum Elongation factor \\
\hline ChELF1a_R & ACCGCCGATCTTGTAGACAT & C. higginsianum Elongation factor \\
\hline ChAve1_QPCR_F5 & CAAGATGCTATGGCAACAATATGAAC & C. higginsianum Ave1 \\
\hline ChAve1_QPCR_R5 & GTCTTGAGGAAAATCTATCGTATTTCTG & C. higginsianum Ave1 \\
\hline BvAct_QPCR_F & GATTTGGCACCACACCTTCT & B. vulgaris actin \\
\hline BvAct_QPCR_R & TCTTTTCCCTGTTTGCCTTG & B. vulgaris actin \\
\hline CbAct_QPCR_F & ACATGGCTGGTCGTGATTTG & C. beticola actin \\
\hline CbAct_QPCR_F & TGTCCGTCAGGAAGCTCGTA & C. beticola actin \\
\hline CbAve1_QPCR_F & ATTCCCTTCAGGCAACCTCT & C. beticola Ave1 \\
\hline CbAve1_QPCR_R & CGGACAAGCTTCGCAATAAT & C. beticola Ave1 \\
\hline VdGapdh_F & CGAGTCCACTGGTGTCTTCA & V. dahliae GAPDH \\
\hline VdGapdh_R & СССTCAACGATGGTGAACTT & V. dahliae GAPDH \\
\hline VdAve1-Fw5 & ATCCTACTATAACCCACCCTACCTTC & V. dahliae Ave1 \\
\hline VdAve1-Rv5 & CATCATATGAGTCCTGAGATAAGATCA & V. dahliae Ave1 \\
\hline XacAve1_QPCR_F & GCAATCGGTTTGCTCTTTTC & X. axonopodis XacPNP \\
\hline XacAve1_QPCR_R & AGCACCGTTATCCCACAGAC & X. axonopodis XacPNP \\
\hline VvAve1-QPCR-F2 & CGTTATAGGTTAAGGTGCCTGAGT & V. vinifera Ave1 \\
\hline VvAve1-QPCR-R2 & TTCATGCTAGGAGAGTGTGAAATG & V. vinifera Ave1 \\
\hline SIAve1_QPCR_F & CGTCGGGGAATCTATTTGTG & S. lycopersicum Ave1 \\
\hline SIAve1_QPCR_R & AAAGCATCCGTTGACAAAGC & S. lycopersicum Ave1 \\
\hline FolAve1_LB_F3 & GGTCTTAAUAACCTAACCTGTATCTAGACCAGAGTGTCT & Left border FolAve1 \\
\hline FolAve1_LB_R3 & GGCATTAAUCAGCAGGTGTTTAGGTATTGTTAAGATAAG & Left border FolAve1 \\
\hline FolAve1_RB_F & GGACTTAAUGTTCTTAGAGCTTAAGGTCAATGTAGC & Right border FolAve1 \\
\hline FolAve1_RB_R & GGGTTTAAUCTGCAGCTTTCACGGGGCTA & Right border FolAve1 \\
\hline ChAve1_LB_F & GGTCTTAAUGGCAGGAGGTCTAGGTGAGA & Left border ChAve1 \\
\hline ChAve1_LB_R & GGCATTAAUCGACTTGAGTTTTGCTGCAC & Left border ChAve1 \\
\hline ChAve1_RB_F & GGACTTAAUAAAGATTTTCGCAGTGCTTCA & Right border ChAve1 \\
\hline
\end{tabular}




\begin{tabular}{|l|l|l|}
\hline ChAve1_RB_R & GGGTTTAAUAGGCTGCAGAATGAGTTTCG & Right border ChAve1 \\
\hline pRF-HU2_Hyg_Fw & CTATTCCTTTGCCCTCGGACGAGTGC & Hygromycin primers \\
\hline pRF-HU2_Hyg_Rv & CGATGTAGGAGGGCGTGGATATGTCC & Hygromycin primers \\
\hline NAT_Fw & CGGGCCGGATTGGTCAAGATTTGC & Nourseothricin primers \\
\hline NAT_Rv & CGATTCGTCGTCCGATTCGTCG & Nourseothricin primers \\
\hline MDB-277 & GACGTTGTAAAACGACGGCCAGTG & Split-marker HYG-F \\
\hline MDB-258 & GGATGCCTCCGCTCGAAGTA & Split-marker HY-R \\
\hline MDB-259 & CGTTGCAAGACCTGCCTGAA & Split-marker YG-F \\
\hline MDB-278 & CACAGGAAACAGCTATGACCATGA & Split-marker HYG-R \\
\hline MDB-1145 & GGCAGGTAGATGACGACCAT & HYG R2 \\
\hline CbAve1 1F & CAAGATTGGGCCTTCGTATG & CbAve1 split-marker left border \\
\hline CbAve1 2R & CACTGGCCGTCGTTTACAACGTCTCTTCAATGGATCCGGACTG & CbAve1 split-marker left border \\
\hline CbAve1 3F & TCATGGTCATAGCTGTTTCCTGTGTGAGTGGGTTTTTGGTTTC & CbAve1 split-marker right border \\
\hline CbAve1 4R & AAGCATACCTCTTCGGCAAA & CbAve1 split-marker right border \\
\hline MDB-760 & CAACGTCTGCCACAAGCTGCT & CbAve1 reverse \\
\hline
\end{tabular}

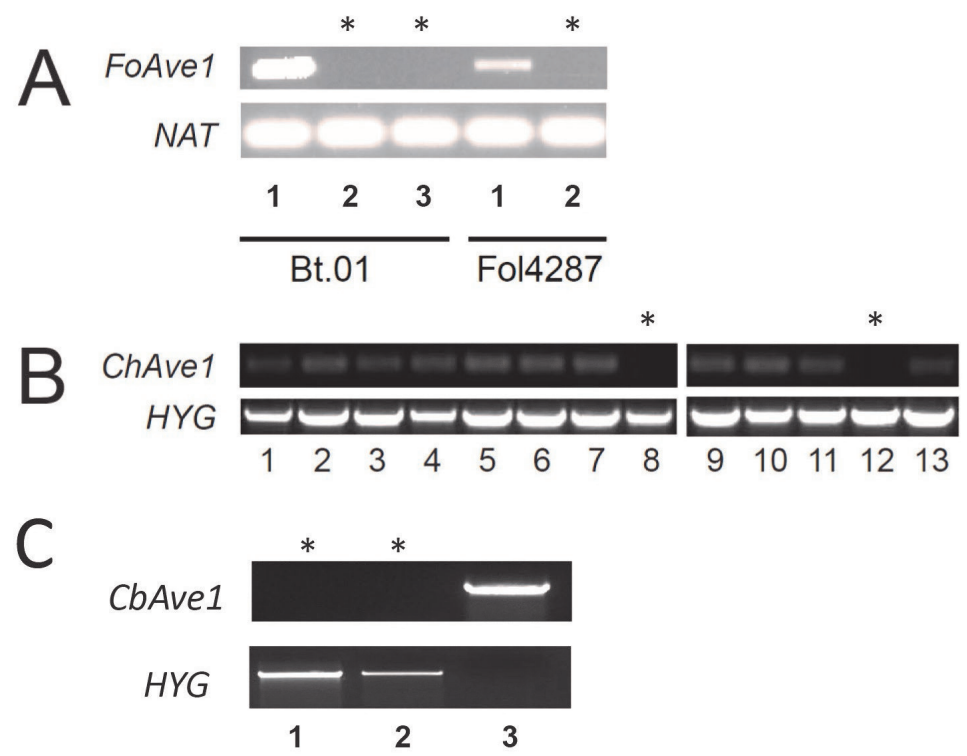

Suppl. Figure 1. Verification of deletion strains. (A) Identification of $F$. oxysporum FoAve1 deletion strains by amplification of FoAve1 and selection marker nourseothricin (NAT) from DNA obtained from transformants. DNA bands were observed at the expected size. Asterisks indicate positive transformants, while transformants that lack an asterisk are ectopic transformants. (B) Identification of C. higginsianum ChAve1 deletion strains by amplification of ChAve1 and selection marker hygromycin (HYG) from DNA obtained from C. higginsianum transformants. DNA bands were observed at the expected size. Asterisks indicate positive transformants, while transformants that lack an asterisk are ectopic transformants. (C) Verification of CbAve1 deletion strains (1 and 2) based on absence of CbAve1 using gene-specific primers and presence of the hygromycin resistance cassette. Amplification on C. beticola Wt gDNA (3) as a control showing the presence of CbAve1 and absence of the hygromycin resistance cassette at the expected size. Asterisks indicate positive transformants. 
A
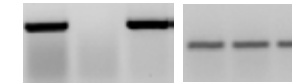

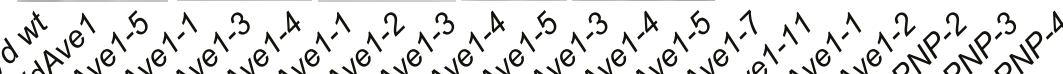

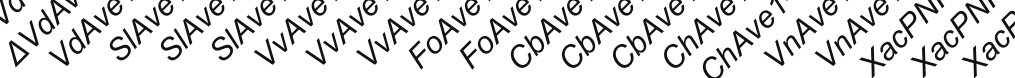
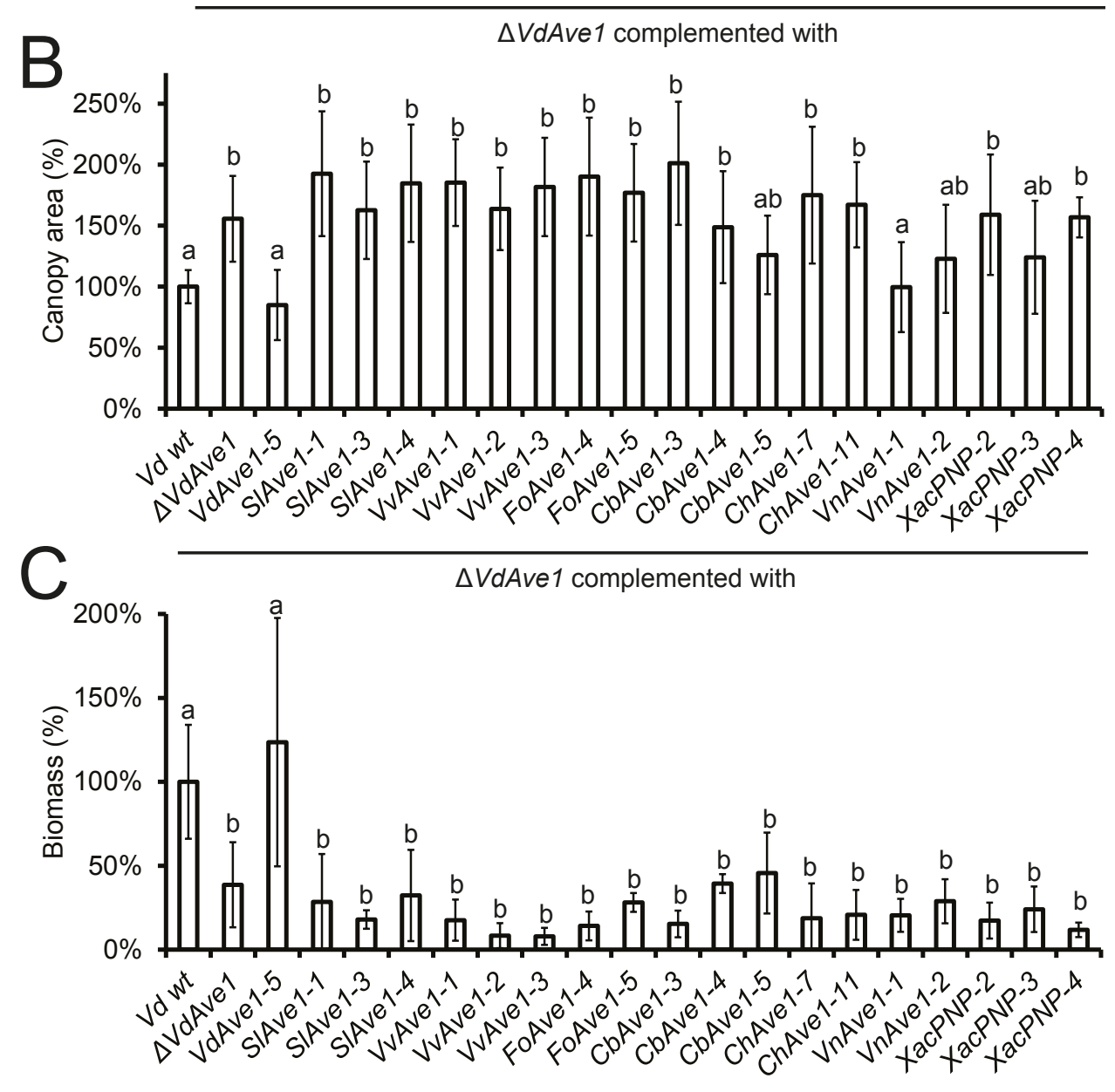

$\Delta V d A v e 1$ complemented with

Suppl. Figure 2. VdAve1 acts in a different manner than Ave1 homologs derived from plant pathogens. $V$. dahliae VdAve1 deletion strain ( $\triangle V d A v e 1)$ complemented with Ave1 homologs derived from V. dahliae (VdAve1), tomato (S. Lycopersicum; S(Ave1), grape (V. vinifera; VvAve1), F. oxysporum (FoAve1), C. beticola (CbAve1), C. higginsianum (ChAve1) and Xanthomonas axonopodis (XacPNP) inoculated on tomato plants. (A) Expression of VdAve1, SlAve1, VvAve1, FoAve1, CbAve1, ChAve1 and XacPNP in a minimum of 2 transformants. (B) Canopy area of tomato plants inoculated with $V$. dahliae strains expressing Ave1 homologs in a minimum of 2 transformants compared to $V$. dahliae wild type (Vd wt) at 14 DPI. (C) Fungal colonization of tomato plants inoculated with $V$. dahliae strains expressing Ave1 homologs compared to V. dahliae wild type (Vd wt) at 14 DPI. Different letter labels indicate significant differences $(P<0.05)$. (D) Photos taken from the side and the top of tomato plants inoculated with $V$. dahliae strains expressing Ave1 homologs compared to $V$. dahliae wild type (Vd wt) at $14 \mathrm{DPI}$. 



\section{Identification of Cercospora beticola necrosis-inducing effector CbNip1}




\section{Abstract}

Cercospora beticola is a hemibiotrophic fungus that causes Cercospora leaf spot disease of sugar beet (Beta vulgaris L.). After an initial, symptom-free, biotrophic phase of colonization, necrotic lesions appear on the host leaves as the fungus switches to a necrotrophic lifestyle. The phytotoxic secondary metabolite cercosporin has been shown to facilitate fungal virulence for several Cercospora spp. However, since cercosporin production and subsequent cercosporininitiated formation of reactive oxygen species is light-dependent, cell death evocation by this toxin is only fully ensured during a period of light. Here, we report the discovery of the effector protein CbNip1 secreted by $C$. beticola that can cause necrosis in the absence of light and therefore may complement light-depended necrosis formation by cercosporin by inducing necrosis during periods of darkness. Infiltration of CbNip1 protein into sugar beet leaves reveals that darkness is essential for full CbNip1-triggered necrosis, as light exposure delayed CbNip1-triggered host cell death. Gene expression analysis during host infection shows that CbNip1 expression is correlated with symptom development in planta. Targeted gene replacement of CbNip1 leads to a significant reduction in virulence indicating the importance of $\mathrm{CbNip1}$ during colonization. 


\section{Introduction}

Cercospora leaf spot (CLS) disease is considered one of the most destructive foliar disease of sugar beet worldwide (1). The causal agent of CLS is the hemibiotrophic fungus Cercospora beticola that belongs to the class of Dothideomycetes (2). In the field, C. beticola over-winters as stromata (1, 3, 4). As C. beticola conidia are airborne, inoculum is dispersed throughout the field by wind, rain and insect transfer $(1,3)$. Upon landing on a sugar beet leaf, spores germinate and grow towards stomata where they form appressoria $(1,5,6)$. These hyphal structures enable the fungus to penetrate and enter the apoplast (7). Once inside the host, C. beticola grows intercellularly and colonizes the mesophyll (1). During these early stages of infection, C. beticola lives a biotrophic lifestyle (1). However, unknown conditions trigger hemibiotrophic fungi to switch from a biotrophic to a necrotrophic lifestyle in which they induce host cell death to complete their lifecycle $(7,8)$

Necrosis-inducing molecules come in many forms and with various modes of actions. For example, necrotrophic effectors, also known as proteinaceous host-selective toxins, depend on the presence of a corresponding target encoded by a susceptibility gene in their host to elicit host cell death (9-12). This interaction is essentially the classic gene-for-gene interaction (13), but instead of providing resistance to the fungus, host cell death serves the necrotrophic needs of the fungus. Therefore, this interaction is also referred to as an inverse gene-forgene interaction (9). For example, the necrotrophic effector SnTox1 of the wheat pathogen Parastagonospora nodorum interacts with Snn1 encoded by a wheat receptor kinase gene, which activates programmed cell death in the host and facilitates a compatible interaction $(11,14,15)$. However, not all necrosis-inducing effectors are dependent on a host receptor to provoke host cell death. A family of Nep1-like proteins (NLPS) has been identified in several oomycetes, fungi and bacteria that elicit a hypersensitive response-like host necrosis (16, 17). The first family member discovered was Nep1 (necrosis and ethylene inducing protein 1), a $24 \mathrm{kDa}$ protein secreted by Fusarium oxysporum that was shown to trigger necrosis and ethylene production in Erythroxylum coca (coca plant) (18). Besides high sequence homology, NLPs share a common NPP1 (necrosis-inducing Phytophthora protein) domain (19). Motteram et al. (2009) (20) reported a NPP1 domain carrying phytotoxic effector called MgNLP that is expressed during infection of the hemibiotrophic pathogen Zymoseptoria tritici, the causal agent of septoria tritici blotch on wheat. Furthermore, necrosis-inducing activity was described as selective since MgNLP induced cell death in Arabidopsis and tobacco but not in wheat. Interestingly, targeted gene replacement of MgNLP did not affect fungal virulence in inoculation studies of susceptible wheat lines (20). Additionally, analysis of $Z$. tritici culture filtrates led to the discovery of two light-dependent phytotoxic proteins, ZtNIP1 and ZtNIP2, whose activities resemble those of host-specific toxins (21). While ZtNIP1 displays homology to the Cladosporium fulvum effector protein Ecp2 that is known to elicit cell death in tomato and tobacco harboring the Cf-ECP2 resistance gene (22), ZtNIP2 was identified to contain a putative MD-2-related lipid-recognition domain hinting at the ability to bind lipids that may have a potential role in innate immunity $(23,24)$. Furthermore, the onset of ZtNIP1 expression during infection matched with necrotic symptom development in planta (21). Recently, the functional ribonuclease Zt6 was discovered in Z. tritici that targets not only plant but also mammalian ribosomal RNA for cleavage in vitro, a feature that makes it highly toxic to wheat, 
tobacco, bacterial and yeast cells (25). Intriguingly, the gene expression pattern of Zt6 during infection is marked by a double expression peak. The first boost in expression occurs at one day post infection, followed by down-regulation during the biotrophic life cycle phase. With onset of the necrotrophic phase at 14 DPI, however, Zt6 gene expression increases again (25).

Besides proteinaceous necrosis-inducing agents, secondary metabolite (SM) effectors have also been reported to elicit cell death in their host. C. beticola is a producer of cercosporin and beticolin, two well-known phytotoxic SMs. Both toxins are only active in the presence of light and show no host specificity $(26,27)$. In multiple Cercospora species, targeted gene disruption mutants that are unable to produce cercosporin displayed reduced virulence which underlines the importance of necrosis induction for the infection process in this genus $(28,29)$. However, no proteinaceous phytotoxin has been reported for $C$. beticola to our knowledge. In this study, we describe the identification of the first proteinaceous $C$. beticola virulence factor, which is able to induce host cell death in the dark and therefore can complement the light-dependent phytotoxins cercosporin and beticolin.

\section{Results}

\section{Necrosis-inducing activity of $C$. beticola culture filtrate}

Due to the hemibiotrophic lifestyle of C. beticola, we hypothesized that the fungus secretes effector proteins during infection that facilitate disease by causing necrosis and at least a portion of these are produced during in vitro growth. Therefore, we cultured C. beticola in different media (PDB and Fries medium) and under different conditions (shaking/still cultures and sampling time points at 3, 5, 7, 12, and 14 days after medium inoculation) in attempts to identify an in vitro condition in which effector proteins are produced (Fig. 1). All culture conditions were tested for the presence of necrosis-inducing activity by infiltrating culture filtrate into sugar beet leaves (Fig. 1). Ultimately, infiltration of culture filtrate of C. beticola grown in Fries medium for seven days, shaking at $120 \mathrm{rpm}$ with exposure to $24 \mathrm{~h}$ light caused clear and repeatable necrosis of the host tissue (Fig. 1). Within 24 hours, the host cells within the infiltration area had entirely collapsed while cells outside the area stayed unharmed. Since C. beticola is known to produce cercosporin and beticolin that are phytotoxic $(26,27)$, culture filtrate was treated with a protease mixture to rule out the involvement of phytotoxic secondary metabolites for this necrosis formation. Proteolysis treatment of culture filtrate abolished necrosis formation, confirming that the necrosis-inducing activity can be attributed to a proteinaceous component of the culture filtrate (Fig. 1). To single out the protein responsible for the necrotic phenotype, the active culture filtrate was fractionized using ion exchange chromatography and single fractions were screened for necrosis-inducing activity by individual infiltration into sugar beet leaves (Fig. 1). The fraction that reproducibly caused necrosis was selected for protein identification using MS/MS analysis.

\section{Effector protein candidate identification}

Based on the analysis of MS/MS data and subsequent protein identity searches in the C. beticola strain 09-40 genome, three candidate effectors were identified: CBET3_03921, CBET3_10646, and CBET3_04765. Of these, CBET3_03921 and CBET3_10646 displayed classic effector 


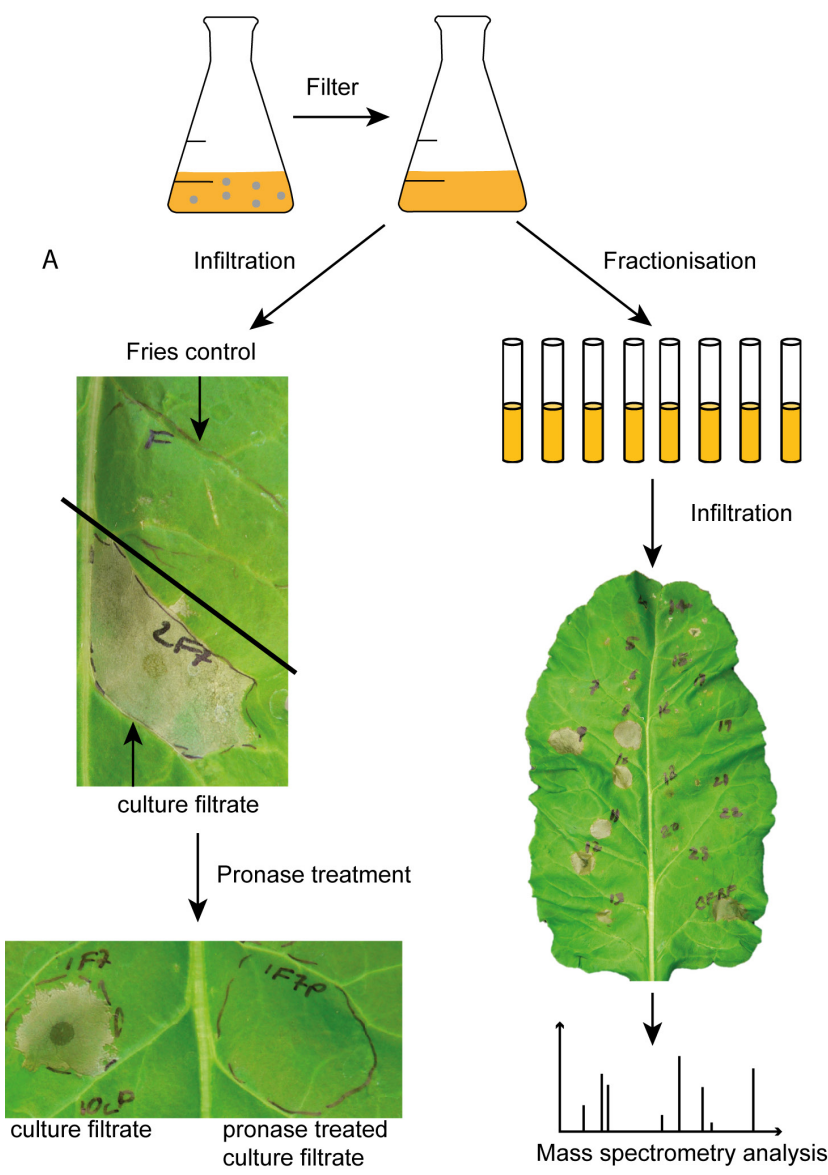

Figure 1. Necrosis-inducing effector identification pipeline. A seven-day old C. beticola 09-40 wildtype strain grown in Fries medium was filtered to remove fungal mycelium. (A) When the culture filtrate was infiltrated into seven-week-old sugar beet leaves, a clear necrotic phenotype was observed after $24 \mathrm{~h}$. Proteolysis treatment eliminated necrosis-inducing activity of the culture filtrate. (B) Culture filtrate was fractionated using ion exchange chromatography and necrosis-inducing activity of individual fractions was assayed by infiltration into sugar beet leaves. All infiltration experiments were repeated at least three times using different sugar beet plants.

characteristics including secretion signals, high cysteine content and low molecular weight (9.2 kDa and $6.6 \mathrm{kDa}$, respectively). In contrast, CBET3_04765 lacked a signal peptide and contained no cysteines and was therefore excluded from further analysis. Interestingly, although CBET3_03921 and CBET3_10646 showed no homology to functionally characterized proteins in the Swiss-Prot database, a large set of homologous, hypothetical proteins was identified for CBET3_03921 when blasted against the NCBI non-redundant database that are mostly derived from Sordariomycetes such as Fusarium spp. and Colletotrichum spp., and two homologous, hypothetical proteins from Colletotrichum spp. were identified for CBET3_10646 
when blasted against the same database. The signal peptide cleavage sites were predicted to be between residues 18 and 19 for CBET3_03921 and between 16 and 17 for CBET3_10646 (Fig. 2). Furthermore, the six cysteine residues found in the 85 -amino acid sequence of the mature CBET3_03921 protein were predicted to form three disulfide bridges (Fig. 2). Although CBET3_10646 is a rather small protein with 59 amino acids, it is predicted to have four disulfide bonds (Fig. 2). On the nucleotide level, each of the two candidate genes had one intron resulting in a coding sequence of 312 bp for CBET3_03921 and 228 bp for CBET3_10646. While no motifs were detectable for CBET3_03921, CBET3_10646 contains an AxxxG motif that may be involved in dimerization (30). Additionally, a $\mathrm{SxxV}(\mathrm{K} / \mathrm{R})$ motif, associated with monocation specificity (Cu+, $\mathrm{Ag}+$, and $\mathrm{Au+}$ ), was also detected $(31,32)$. While a $\mathrm{SxxV}(\mathrm{K} / \mathrm{R})$ motif was previously reported to occur in combination with a CxGxxxxDCP metal binding loop, CBET3_10646 appears to only be harboring the monocation specificity domain without the metal binding loop motif.

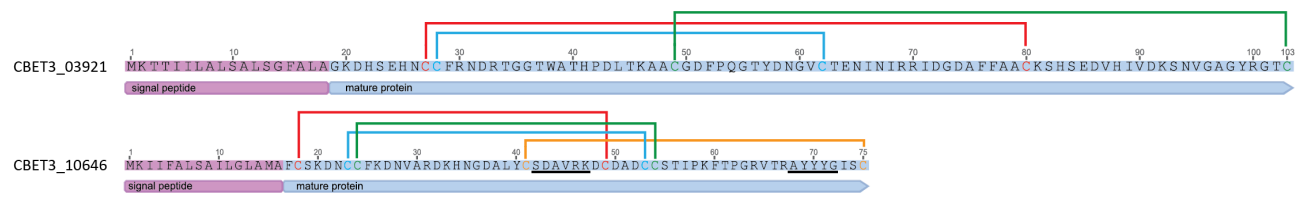

Figure 2. Peptide sequence of CBET3_03921 (CbNip1) and CBET3_10646. Both candidates display classic effector characteristics such as signal peptides and predicted disulfide bonds (highlighted). Underlined in CBET3_10646 sequence are a conserved SxxV(K/R) motif associated with monocation specificity and an AxxxG motif shown to be involved in dimerization.

\section{Heterologous expression of effector protein candidates and phenotype upon infiltration}

To further characterize the candidate necrosis-inducing effectors, CBET3_03921 protein was produced heterologously in Escherichia coli and infiltrated into sugar beet leaves that were subsequently kept in a growth chamber with a 10-hour light cycle. Unlike the response from the culture filtrate, no phenotype was observed for CBET3_03921 at $24 \mathrm{~h}$ (Fig. 3A). However, after $48 \mathrm{~h}$ the infiltration area of CBET3_03921 started to appear slightly chlorotic while the empty vector control remained unchanged (Fig. 3 A). Chlorosis of the CBET3_03921 infiltrated area increased over time until it turned necrotic. Since light is critical for the activity of $C$. beticola SM effectors cercosporin and beticolin, we questioned whether light may play a role in the activity of CBET3_03921. To evaluate, we infiltrated CBET3_03921 protein into sugar beet leaves that were subsequently placed in a growth chamber in $24 \mathrm{~h}$ darkness. Incubation of CBET3_03921 infiltrated leaves in the dark resulted in clear necrosis of the complete infiltration area by 3 DPI (Fig. 3 B). To assess the stability of CBET3_03921, we incubated the protein and empty vector control at $50^{\circ} \mathrm{C}$ or $100^{\circ} \mathrm{C}$ for 30 minutes, after which proteins were infiltrated into sugar beet leaves and subsequently shielded from light exposure. While exposure to $100^{\circ} \mathrm{C}$ abolished necrosis-formation, samples treated with $50^{\circ} \mathrm{C}$ were still able to cause necrosis (Fig. 3 C). Furthermore, infiltrations of CBET3_03921 into Nicotiana benthamiana led to the same necrotic phenotype, indicating that CBET3_03921 mode-of-action is not host specific (Fig. 3 D). Due to its necrosis-inducing character, we renamed CBET3_03921 to CbNip1 (CbNip for Cercospora beticola necrosis-inducing protein 1). 
A

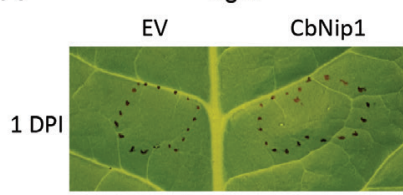

2 DPI

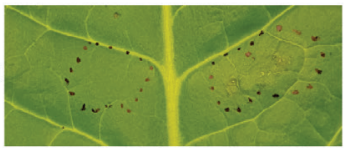

$3 \mathrm{DP}$

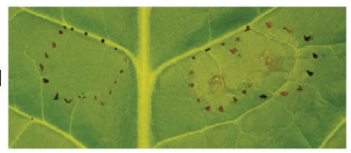

C 3 DPI in the dark

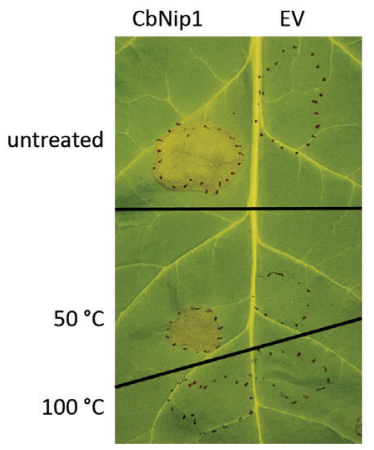

B
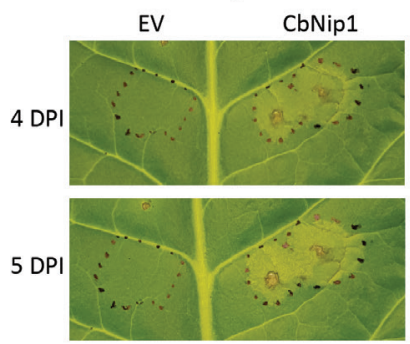

$6 \mathrm{DPI}$

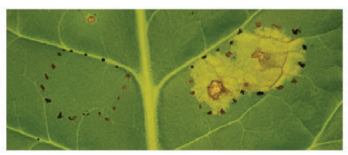

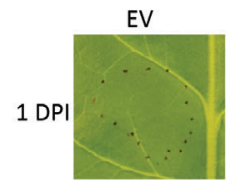
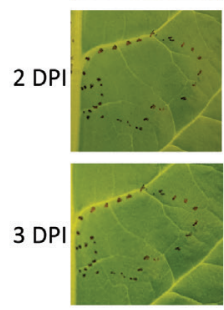

Dark

CbNip1

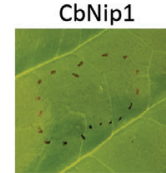

D $3 \mathrm{DPI}$ in the dark ( $N$. benthamiana)

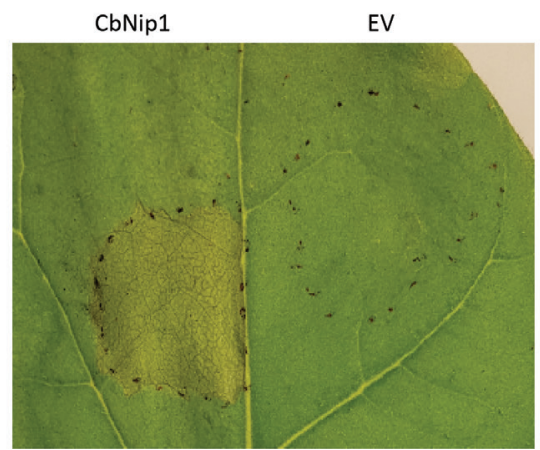

Figure 3. Necrosis-inducing phenotype of CbNip1 protein. (A) Chlorosis/necrosis development after infiltration of CbNip1 into sugar beet leaf exposed to a $10 \mathrm{~h} / 14 \mathrm{~h}$ light/dark cycle for 6 days, and empty vector sample (EV) infiltration served as a control. (B) Necrosis development after infiltration of CbNip1 into a sugar beet leaf kept in $24 \mathrm{~h}$ darkness, and an empty vector infiltration that served as a control. (C) Treatment of CbNip1 and an empty vector exposed to $50^{\circ} \mathrm{C}$ for $30 \mathrm{~min}$ did not affect necrosis-inducing activity of $\mathrm{CbNip} 1$ while treatment of both samples at $100^{\circ} \mathrm{C}$ for $30 \mathrm{~min}$ abolished necrosis-induction. Untreated samples served as controls. (D) Necrosis formation after infiltration of CbNip1 into a N. benthamiana leaf. An empty vector control sample served a control. All infiltration experiments were repeated at least three times using different sugar beet plants.

We were unable to produce CBET3_10646 in sufficient amounts in either Pichia pastoris or E. coli. Therefore, chemically synthesized CBET3_10646 protein was used for infiltration into sugar beet leaves. In contrast to CbNip1, no phenotype was visible for the conditions tested, which included light/dark exposure, refolding of the protein and the supplementation of trace elements. Consequently, CBET3_10646 was excluded from further analysis.

\section{In planta gene expression profile of $\mathrm{CbNip1}$ matches necrotic lesion development}

To determine whether CbNip1 expression pattern during C. beticola colonization also matches necrosis emergence in planta, we inoculated sugar beet plants with a $C$. beticola wild type 
250

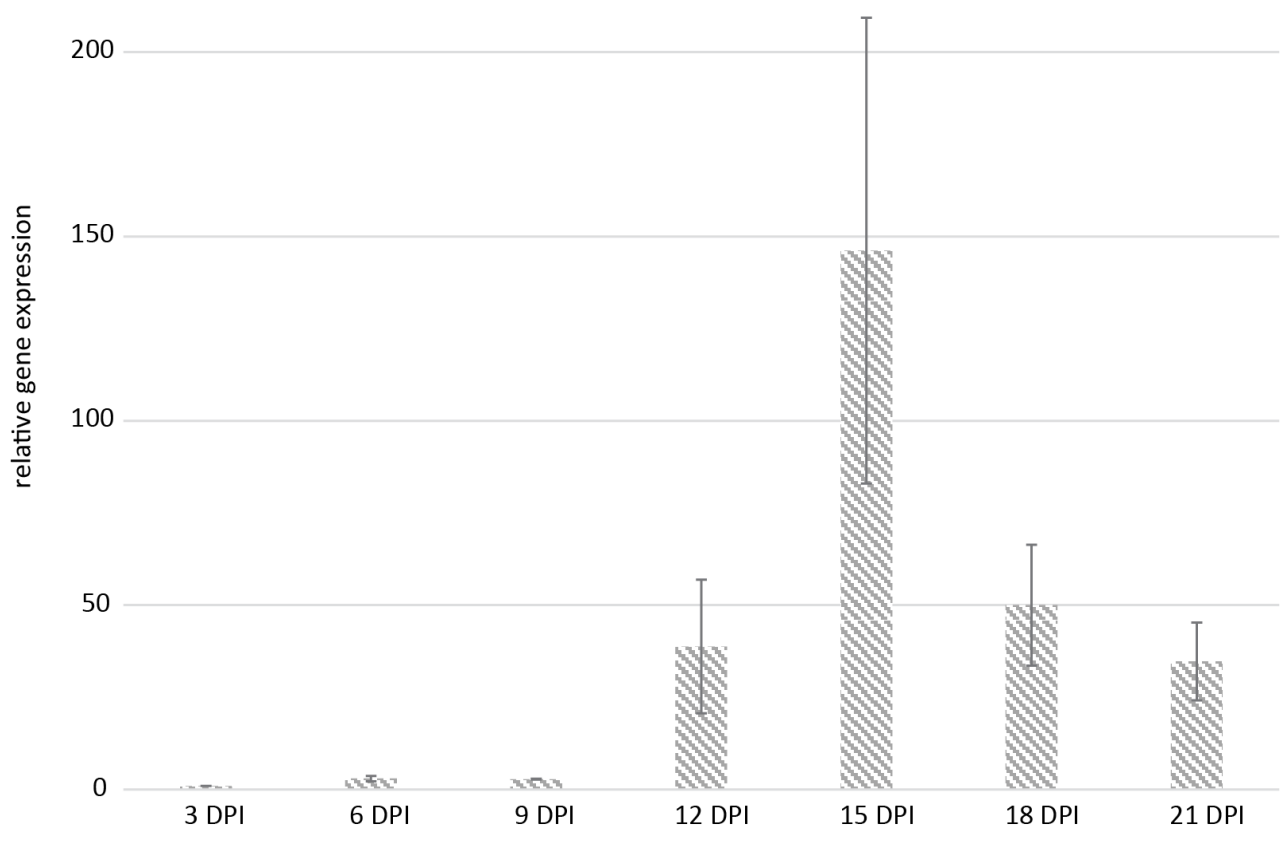

Figure 4. CbNip1 gene expression during $C$. beticola infection on sugar beet. Gene expression profile of $C b N i p 1$ during 09-40 C. beticola wild type strain infection course at 3, 6, 9, 12, 15, 18 and 21 days post infection (DPI). CbNip1 gene expression was normalized to $C$. beticola actin gene expression. Error bars indicate the standard error of three biological replicates.

strain and harvested leaf samples at 3, 6, 9, 12, 15, 18, and 21 days post inoculation (DPI). Gene expression analysis revealed that CbNip1 is minimally expressed at early time points (Fig. 4). However, from 12 DPI onwards CbNip1 expression increased until peaking at 15 DPI. Interestingly onset of CbNip1 upregulation at 12 DPI matched symptom development on the sugar beet leaves (Suppl. Fig. 2). At 15 DPI, many single necrotic spots were visible while CbNip1 expression reached its peak. However, with progressing necrosis expansion in planta, CbNip1 experiences a steady downregulation again from 18 DPI onwards (Fig. 4).

\section{CbNip1 is a virulence factor}

To investigate whether CbNip1 is required for full C. beticola virulence, we inoculated sugar beet plants with wild-type C. beticola and gene deletion mutants lacking CbNip1. In addition to visible symptom assessment in planta, fungal biomass was measured using qPCR for each treatment individually to determine the level of fungal colonization of the host plants. While severe infection 

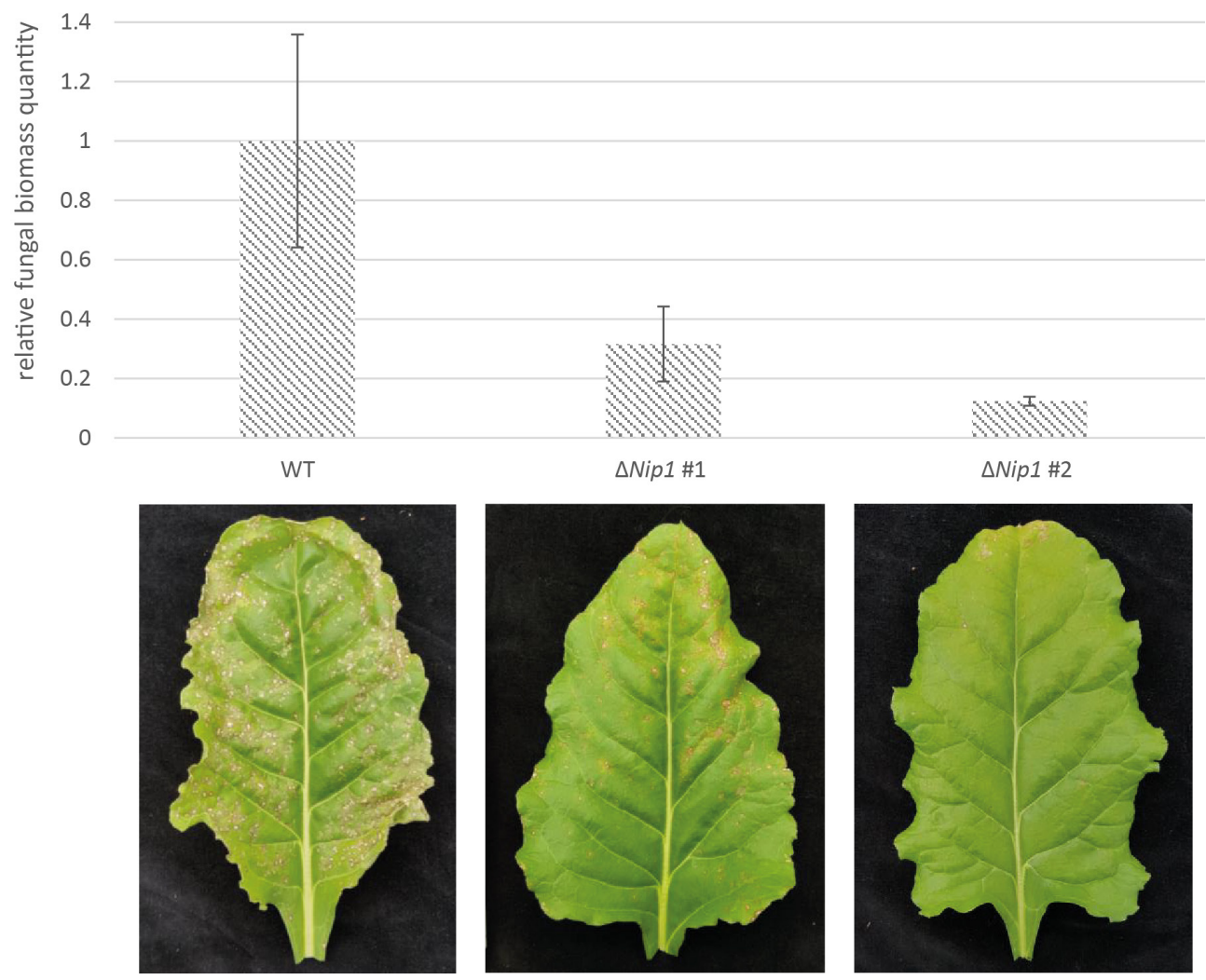

Figure 5. Fungal biomass quantification of $C$. beticola 09-40 wild type strain and two individual $\Delta C b N i p 1$ mutants. Sugar beet plants inoculated with $C$. beticola strains at 13 DPI with photos showing respective disease severity below. Error bars represent standard error of five biological replicates.

symptoms were displayed by sugar beet plants inoculated with wild type C. beticola, highly-reduced symptom formation was observed for plants inoculated with two individual $\triangle C b N i p 1$ strains (Fig. 5). In agreement with the noticeable difference in in planta phenotype of wild type and $\triangle C b N i p 1$ strains, evaluation of fungal biomass showed reduced fungal colonization in plants infected with $\triangle C b N i p 1$ compared to high levels of fungal biomass found in sugar beet plants inoculated with the wild type $C$. beticola strain. In contrast, $\triangle C B E T 3 \_10646$ mutants did not exhibit a virulence penalty when compared with the progenitor wild type C. beticola strain (data not shown).

\section{Discussion}

C. beticola is a hemi-biotrophic fungus that is dependent on necrosis formation during infection (33) and known to utilize the secondary metabolite effector cercosporin to cause host cell death (26). Here, we report the identification of the novel C. beticola necrosisinducing effector protein CbNip1. 
By searching for in vitro parameters that trigger C. beticola to secrete effector proteins, we found growth conditions under which $C$. beticola produces proteinaceous effectors that cause necrosis upon infiltration into sugar beet leaves within 24 hours. While infiltration of pure CbNip1 into sugar beet leaves took $48 \mathrm{~h}$ to lead to visible necrosis, the timing difference in necrosis formation is likely due to the presence of multiple necrosis-inducing effectors besides CbNip1 in the culture filtrate. Interestingly, CbNip1 activity was more pronounced in the dark. Besides CbNip1, fractionation of the culture filtrate with subsequent mass spectrometry analysis of the necrosis-inducing fraction identified the presence of two other proteins CBET3_10646 and CBET3_04765, of which CBET3_04765 was excluded for further analysis due to the lack of typical effector characteristics.

For functional analysis, CbNip1 was heterologously produced and infiltrated into sugar beet leaves. We found that the full potential of CbNip1 to induce host cell death was dependent on the absence of light. Light is known to influence Cercospora zeae-maydis infection capability as the ability to find stomata and form appressoria is abolished in the dark (34). Plants are also impacted by light in various ways including alteration of leaf physiology $(35,36)$. Furthermore, studies on host resistance responses have demonstrated that light is required for the full cascade of plant resistance responses (36-38). Based on micro-array expression profiling of the C. beticola - sugar beet interaction, 571 sugar beet genes were induced including pathogenesis-related (PR) genes and genes involved in lignin and alkaloid biosynthesis at the onset of necrotic symptom formation (33). While the products of these defense-associated genes could potentially impede CbNip1 function in the presence of light, PR genes have been shown to be repressed in the dark (36-38). While necrotrophic effectors such as SnTox1 have been reported exploit interactions with defense-associated genes such as specific plant receptors for host cell death induction (11,14), in case of CbNip1, necrosis is not only induced in sugar beet but also in the non-host N. benthamiana. Therefore, it is likely that CbNip1 function may not be sugar beet specific via interaction with a corresponding receptor protein but rather display general toxicity to plants. For example some effectors modulate targets in their host but potentially also in other plants for necrosis induction. This mode-of-action has been observed for the small sRNase Zt6 of Z. tritici that displays universal cytotoxicity by cleaving plant and mammalian ribosomal RNA (25). Nevertheless, necrosis formation could also be the result of cell wall degrading enzyme activity as cell wall degrading enzymes of various fungal pathogens have been found to be essential for fungal virulence (39-41).

While a necrotic phenotype is observed for CbNip1, the other effector candidate CBET3_10646 failed to induce any phenotype under tested conditions. Since CBET3_10646 has domains associated with dimerization and monocation specificity, the inability to induce necrosis may be due to the absence of the right cofactor. As CBET3_10646 was chemically synthesized, it is possible that due to its monocation specificity associated domain, the addition of trace elements (including copper) as present in the Fries media of the initial culture filtrate might activate CBET3_10646 function. However, the supplementation of metal ions to CBET3_10646 did not lead to phenotype formation in sugar beet leaves. Moreover, infiltration of CBET3_10646 with CbNip1 did not obviously enhance CbNip1-induced necrosis. Further research is required to identify the allied co-factors for CBET3_10646, if any. Since CBET3_10646 mutants did not exhibit a virulence penalty in planta, CBET3_10646 may not be an effector for C. beticola. 
In accordance with CbNip1 necrosis-inducing ability, we have found that necrotic symptom development in planta correlates with up-regulation of CbNip1 expression (Fig. 4; Suppl. Fig. 2). Induction of host necrosis during the biotrophic phase is not likely beneficial for the fungus, therefore it is not surprising that CbNip1 is minimally expressed at early infection time points (3 DPI to 9 DPI). An increase in CbNip1 expression and the development of necrotic lesions occurred simultaneously, suggesting that CbNip1 is linked to the switch from biotrophic to necrotrophic life style of the fungus. Once necrosis formation is ongoing and existing necrotic lesions start to fuse, CbNip1 expression is reduced again to a similar level as observed in the initial cell death induction phase at 12 DPI, indicating that necrosis induction by CbNip1 may still be important at later time points. Interestingly, CbNip1 expression pattern is similar to expression patterns of other necrosis-inducing effectors from different protein families found in the hemibiotroph Z. tritici. ZtNIP1 showed an expression pattern where gene upregulation matched onset of symptom development in planta (21). Similarly, the Z. tritici Nep-1 like protein MgNLP peaked towards the end of the biotrophic phase before necrotic lesions were visible (20). However, there are also examples of contrasting expression patterns to CbNip1. For example the expression of Zt6 in planta that is characterized by a double peak likely attributed to a double functionality (25).

Since $C$. beticola requires necrotic plant tissue to complete its life cycle (33), we determined whether CbNip1 was also essential for fungal virulence. We found that site-directed CbNip1 deletion mutants are impeded in virulence compared to the wild type $C$. beticola strain. Not only did plants inoculated with $\triangle C b N i p 1$ mutants develop fewer $C$. beticola-specific lesions, biomass determination revealed there was less fungal biomass in plant tissue compared to the progenitor wild type (Fig. 5). Taken together, this indicates that CbNip1 plays an important role in C. beticola virulence. As mentioned earlier, C. beticola produces the secondary metabolite cercosporin and a family of phytotoxins called beticolins, both of which are able to cause cell death in the presence of light $(26,27,42,43)$ and cercosporin was shown to be a virulence factor for several Cercospora species $(28,29)$. Since lightactivation is essential for cercosporin and beticolin functionality, they are likely not active in the dark. With the secretion of CbNip1 however, C. beticola may be defying this light-associated limitation by utilizing additional necrosis-inducing agents to cover both light and dark conditions to achieve maximal host cell death to complete its life cycle.

In conclusion, we have shown that C. beticola secretes the effector protein CbNip1 during infection that in the absence of light has the ability to cause necrosis upon infiltration into sugar beet leaves within 48 hours. Furthermore, CbNip1 expression in planta correlates with necrotic symptom appearance during C. beticola sugar beet infection. Targeted gene replacement of CbNip1 led to a reduction in virulence, indicating that $\mathrm{CbNip1}$ is a virulence factor for $\mathrm{C}$. beticola. As CbNip1 has no obvious homology to other proteins in public databases, future studies will be directed to identify the CbNip1 mode-of-action. Usually, studies on pathogen - host plant interactions focus on processes in the presence of light, however it may be interesting to understand how this interaction is altered in the dark, a vital condition for unhampered CbNip1 function. Consequently, CbNip1 is a fungal virulence factor that is hypothesized to take advantage of the reduced host plant defense response level due to the absence of light. Further analysis of yet unknown functional motifs of CbNip1 as well as localization studies will help to shed light on the biology of CbNip1. 


\section{Materials and Methods}

\section{Fungal strains}

C. beticola wild type strain 09-40 was isolated from leaf material collected from a sugar beet field in the Red River Valley, USA in 2009. The fungus was kept at $22^{\circ} \mathrm{C}$ on potato dextrose agar (PDA; Difco, Sparks, USA) and fungal site-directed gene deletion mutants in a 09-40 background on PDA amended with 150 MM hygromycin B (Duchefa, Haarlem, NL).

\section{Culture filtrate preparation and infiltration}

A $5 \mathrm{~mm}$ plug was taken from the actively growing zone of C. beticola wild type strain 09-40 on PDA and used to inoculate a $250 \mathrm{ml}$ conical flask filled with $100 \mathrm{ml}$ of Fries media (44). After seven days of incubation at 120 rpm under 24 hour light conditions, the liquid culture was run through two layers of Miracloth (EMS Millipore Corp., Billerica, USA) to clear it of fungal mycelium and subsequently filter-sterilized with a $0.45 \mu \mathrm{m}$ Filtropur membrane (Sarstedt, Nümbrecht, Germany). Approximately 30 to $50 \mu \mathrm{l}$ of sterile culture filtrate were infiltrated into the leaves of 7-week-old sugar beet plants of the variety C093 (formerly 86RR66) using a $1 \mathrm{ml}$ needleless syringe. Infiltration experiments were repeated at least three times with multiple individually produced culture filtrates. Plants were kept in a greenhouse chamber with an average temperature of $26^{\circ} \mathrm{C}$ during the day and approximately $17^{\circ} \mathrm{C}$ during the night. Chambers were equipped with additional lighting to ensure 16 hours of light a day. To confirm the proteinaceous nature of the necrosis-inducing agent, $50 \mu \mathrm{l}$ of MOPS buffer (1M, pH 7.5) and $25 \mu \mathrm{l}$ of pronase ( $1 \mathrm{mg} / \mathrm{ml}$ ) (Sigma, St Louis, USA) or water as control was added to $425 \mu \mathrm{l}$ of culture filtrate and incubated at $22{ }^{\circ} \mathrm{C}$ for $4 \mathrm{~h}$. Subsequently, samples were infiltrated into sugar beet leaves as described above.

\section{Culture filtrate fractionation}

Culture filtrate was partially purified as described in (45). In short, $100 \mathrm{ml}$ of 7-day-old C. beticola wild type strain 09-40 grown in Fries media were first filter-sterilized and then dialyzed against water using a $3.5 \mathrm{kDa}$ molecular weight cutoff dialysis membrane (Fisher Scientific, Pittsburgh, USA). The next day, the dialyzed culture filtrate was loaded onto a HiPrep SPXL 16/10 cation exchange column (GE Healthcare Piscataway, USA) using the ÄKTA prime plus (GE Healthcare, Piscataway, USA) liquid chromatography system. After a washing step with $50 \mathrm{ml}$ of $20 \mathrm{mM}$ sodium acetate buffer $\mathrm{pH} 5.0,5 \mathrm{ml}$ fractions were collected during gradient elution of $0-300$ mM sodium chloride plus $20 \mathrm{mM}$ sodium acetate pH 5.0 at a flow rate of $5.0 \mathrm{ml} / \mathrm{min}$ over $20 \mathrm{~min}$. Collected fractions were individually infiltrated into 7-week-old sugar beet plants of the variety C093 (formerly 86RR66) and screened for necrotic phenotype. Fractionation and infiltration experiments were repeated at least three times.

\section{Preparation for MS/MS analysis}

The fraction that repeatedly caused necrosis was loaded onto a precast $16.5 \%$ tris-tricine polyacrylamide gel (Bio-Rad, Hercules, USA). Protein spots were excised and sent to the Center 
for Mass Spectrometry and Proteomics at the University of Minnesota for trypsin digestion and subsequent LCMS analysis. Peptide mass fingerprints and peptide sequence information were used to search for protein identity using the annotated C. beticola 09-40 genome (46).

\section{gDNA extraction, RNA extraction, cDNA synthesis}

Genomic DNA was extracted using a modified version of the microprep protocol published by Fulton et al. (1995), replacing chloroform:isoamyl alcohol (24:1) with phenol:chloroform:isoamyl alcohol (25:24:1).

RNA extraction followed the Trizol method (Ambion, Carlsbad, USA) according to the manufacturer's protocol and subsequently cleaned up three times using the RNase-Free DNase Set (Qiagen, Hilden, GER) according to Appendix E of the RNase Mini Handbook 06/2012. For CDNA synthesis, 1 $\mathrm{\mu g}$ of total RNA was used with the SuperScript III reverse transcriptase kit (Invitrogen, Carlsbad, USA) following the manufacturer's protocol.

\section{Sequence analyses}

Signal peptides (if present) were determined with SignalP online tool (ttp://www.cbs.dtu.dk/services/ SignalP) while disulfide bonds were predicted using DISULFIND (http://disulfind.dsi.unifi.it/).

\section{RT-PCR of CbNip1}

Quantitative RT-PCR was performed in triplicate using the SensiMix SYBR Hi-Rox kit (Bioline, Luckenwalde, Germany) with an ABI7300 PCR machine (Applied Biosystems, the Netherlands) and CDNA of each time point for gene expression analysis or gDNA of each treatment for fungal biomass quantification. All reactions were done in triplicate and primers are listed in Suppl. Table S1. Real-time PCR conditions started with a denaturation step of $10 \mathrm{~min}$ at $95^{\circ} \mathrm{C}$, followed by denaturation for $15 \mathrm{~s}$ at $95^{\circ} \mathrm{C}$, annealing for $30 \mathrm{~s}$ at $60^{\circ} \mathrm{C}$, and extension for $30 \mathrm{~s}$ at $72^{\circ} \mathrm{C}$ for 30 cycles. Water as template controls were included for all qPCR runs. With C. beticola actin as a reference gene for the gene expression study, relative gene expression of three biological repetitions were calculated in comparison to the earliest measured time point using the Pfaffl method (47). Variation in gene expression was calculated using the standard error of the means of three biological replicates. Biomass was determined using the $\Delta \Delta \mathrm{Ct}$ method (48) relative to the average value of the wild type inoculated sugar beet plants. Error bars indicate standard error of variation between three individual biological replicates. Primers are listed in Suppl. Table S1.

\section{Vector construction and protein production in E. coli}

For heterologous protein expression in E. coli, CbNip1 was amplified with GoTaq Long PCR Master Mix (Promega, Madison, USA) from C. beticola 09-40 wild type cDNA using primers MKE-78/77 (Suppl. Table 1), respectively. Amplicons and pET Sumo vector (Invitrogen, Carlsbad, US) were digested with ECORI and Notl and followed by ligation of the fragments into the double 
digested pET Sumo vector with T4 DNA ligase (NEB, Beverly, USA) and cloned into E. coli DH5a. Plasmids carrying the correct CbNip1 coding sequence were verified by sequencing (Eurofins Genomics, Ebersberg, Germany) and as well as an empty pET Sumo vector subsequently cloned into E. coli Origami strain (DE3) strain.

For heterologous protein expression, $1000 \mathrm{ml}$ of LB were inoculated with a $20 \mathrm{ml}$ overnight LB plus kanamycin $50 \mathrm{\mu g} / \mathrm{ml}$ culture with either CbNip1 pET expression construct or the empty vector control and grown at $37^{\circ} \mathrm{C}$ shaking at $200 \mathrm{rpm}$ until reaching an $\mathrm{OD}_{600}$ between 0.6-0.8. Protein production was induced with $0.05 \mathrm{mM}$ Isopropyl $\beta$-D-1-thiogalactopyranoside (IPTG) final concentration and kept growing at $20^{\circ} \mathrm{C}$ shaking at $200 \mathrm{rpm}$ for 24 hours. Cells were pelleted, snap frozen with liquid nitrogen and then lysed with $20 \mathrm{ml}$ lysis buffer containing $50 \mathrm{mM}$ Tris-HCL pH 8.5 (Invitrogen, Carlsbad, USA)and 150 mM NaCl (Sigma, St Louis, USA), 10\% glycerol (Amresco, Solon, USA), 6 mg/ml lysozyme from chicken egg white (Sigma, St Louis, USA), 2 mg/ml sodium deoxycholate (Sigma, St Louis, USA), $0.625 \mathrm{mg} / \mathrm{ml}$ Deoxyribunuclease I from bovine pancreas (Sigma, St. Louis, USA), and one cOmplete protease inhibitor pill (Sigma, Mannheim, Germany). After the cultures were kept on ice for $1 \frac{1 / 2}{2}$ hours, cells debris was spun down for one hour at 14000 rpm at $4^{\circ} \mathrm{C}$ and the soluble protein fraction was processed for protein purification.

\section{Protein purification}

In E. coli heterologously produced protein samples were loaded at $1 \mathrm{ml} / \mathrm{min}$ onto a column packed with $2 \mathrm{ml}$ of Ni Superflow Resin (Clontech, Mountain View, US) for purification. After a washing step with wash buffer (50 mM Na2HPO4 (Merck, Darmstadt, Germany), 300 mM NaCl (Sigma, St Louis, USA), $40 \mathrm{mM}$ imidazole (Merck, Darmstadt, Germany) at $2 \mathrm{ml} / \mathrm{min}$ to wash out contaminative $E$. coli native proteins, SUMO-tagged CbNip1 or the Sumo tag alone obtained from the empty vector sample were eluted with elution buffer 50 mM Na2HPO4 (Merck, Darmstadt, Germany), 300 mM NaCl (Sigma, St Louis, USA), 40 mM imidazole (Merck, Darmstadt, Germany). Elution samples were dialyzed with a Spectra/Por Dialysis Membrane with MWCO of 3,500 (Spectrum Laboratories, Rancho Dominguez, USA) against $200 \mathrm{mM} \mathrm{NaCl}$ containing ULP-1 enzyme to cleave off the SUMO tag at $4{ }^{\circ} \mathrm{C}$ overnight without agitation. The next day samples were run through the Nickle bead column with the same setup as before at $1 \mathrm{ml} / \mathrm{min}$ to allow cleaved off SUMO tags to bind to the Nickle beads. Flow-through was collected and again dialyzed for 24 hours against $200 \mathrm{mM}$ $\mathrm{NaCl}$. Samples were concentrated with Amicon Ultra-15 centrifugal filter unit with an Ultracel-3 membrane (Millipore, Billerica, USA) with a $3 \mathrm{kDa}$ cut off. For visualization, five $\mu$ l of protein sample were loaded on Mini-PROTEAN TGX stain free precast gels (Biorad, Hercules, USA).

\section{Refolding and preparation of CBET3_10646 for sugar beet leaf infiltration}

Synthesized mature CBET3_10646 purchased from GeneScript (Piscataway, USA) was dissolved in MQ water to $3 \mathrm{mg} / \mathrm{ml}$. For refolding, oxidized glutathione (Sigma, St Louis, USA) and reduced glutathione (Sigma, St Louis, USA) were added to $1 \mathrm{mg} / \mathrm{ml}$ of CBET3_10646 to an end ratio of 5:1 $\mathrm{mM}$ and incubated overnight. $M Q$ water treated with the same glutathione ratio served as a control. To see whether the addition of trace elements leads to activation of necrosis-inducing 
activity of CBET3_10646, $1 \mu \mathrm{l}$ of trace element stock were added to $0.5 \mathrm{ml}$ of protein sample or water control to a trace element end concentration as found in Fries media used for the culture filtrate experiment.

\section{Protein infiltration}

Sugar beet of the variety C093 (formerly 86RR66) were grown in the climate chamber at $21^{\circ} \mathrm{C}$ with 10 hours light with 10 lux and $70 \%$ humidity. After 7 weeks approximately 30 to $50 \mu \mathrm{l}$ of purified protein ( 2 mg ml-1) or empty vector (Suppl. Fig. 1) were infiltrated into the leaves using a $1 \mathrm{ml}$ needleless syringe and the infiltration area was marked with a marker. Dark-treated leaves were wrapped in aluminum foil to prevent light exposure. For this experiment, CBET3_10646 and three individually produced and purified CbNip1 samples were infiltrated at least three times.

\section{Deletion mutants}

Site-directed gene deletion mutants were generated following the split-marker PEG-protocol described in Bolton et al. (2016) (49). Primers are listed in Suppl. Table S1. Gene deletion was verified with two different approaches, by absence of PCR product using gene specific forward and revere primers and by presence of an amplicon for a forward primer designed upstream of the $5^{\prime}$ flanking region of the target gene and a reverse primer annealing to the hygromycin resistance cassette.

\section{Inoculation assay}

Spore formation of C. beticola wild type and two individual deletion mutants was induced on CV8 agar plates as previously described (50). Spores were harvested and adjusted to a concentration of $1 \times 105$ spores per $\mathrm{ml}$ and spore suspension was equally spayed on the lower sides of the leaves of 7-week-old sugar beet plants of the variety C093 (formerly 86RR66). Inoculated plants were kept in a humidity tent inside the greenhouse chamber with about $27^{\circ} \mathrm{C}$ and $90 \%$ humidity for 5 days after which the tent was removed and plants were exposed to $22^{\circ} \mathrm{C}$ with a $16-\mathrm{h} / 8$-h day/night cycle. For fungal biomass analysis three leaves of two plants for five repetitions were harvested at 13 DPI (days post infection) and instantly snap frozen while plants for gene expression analysis were harvested 3 , $6,9,12,15,18$, and $21 \mathrm{DPI}$ using three leaves of two plants in three repetitions.

\section{Acknowledgements}

M.D. Bolton is supported by USDA - ARS CRIS project 3060-22000-049 and grants from Beet Sugar Development Foundation and the Sugar Beet Research and Education Board of MN and ND. M.K. Ebert was supported by NWO grant 833.13.007. This work was financially supported by an EMBO Long-Term Fellowship (ALTF 359-2013) and a postdoctoral fellowship of the Research Foundation Flanders (FWO 12B8116N) to R. de Jonge. 


\section{References}

1. Weiland J \& Koch G (2004) Sugarbeet leaf spot disease (Cercospora beticola sacc.). Molecular plant pathology 5(3):157-166

2. Bolton MD, et al. (2012) Evaluation of the potential for sexual reproduction in field populations of Cercospora beticola from USA. Fungal Biology 116(4):511-521.

3. Khan MFR \& Khan J (2010) Survival, spore trapping, dispersal, and primary infection site for Cercospora beticola in sugar beet. Cercospora leaf spot of sugar beet and related species, eds Lartey RT, Weiland JJ, Panella L, Crous PW, \& Windels CE (APS Press, St. Paul).

4. Solel Z \& Minz G (1971) Infection process of Cercospora beticola in sugarbeet in relation to susceptibility. Phytopathology 61:463-466.

5. Rathaiah Y (1977) Stomatal tropism of Cercospora beticola in sugarbeet. Phytopathology 67(3):358-362.

6. Feindt F, Mendgen K, \& Heitefuß R (1981) Feinstruktur unterschiedlicher Zellwandreaktionen im Blattparenchym anfälliger und resistenter Rüben (Beta vulgaris l.) nach Infektion durch Cercospora beticola sacc.

7. Steinkamp MP, Martin SS, Hoefert LL, \& Ruppel EG (1979) Ultrastructure of lesions produced by Cercospora beticola in leaves of Beta vulgaris. Physiological Plant Pathology 15(1):13-26.

8. Horbach R, Navarro-Quesada AR, Knogge W, \& Deising HB (2011) When and how to kill a plant cell: Infection strategies of plant pathogenic fungi. J Plant Physiol 168(1):51-62.

9. Friesen TL, Meinhardt SW, \& Faris JD (2007) The Stagonospora nodorum-wheat pathosystem involves multiple proteinaceous host-selective toxins and corresponding host sensitivity genes that interact in an inverse genefor-gene manner. The Plant Journal 51(4):681-692.

10. Friesen TL, Faris JD, Solomon PS, \& Oliver RP (2008) Host-specific toxins: Effectors of necrotrophic pathogenicity. Cellular microbiology 10(7):1421-1428.

11. Shi G, et al. (2016) The hijacking of a receptor kinase-driven pathway by a wheat fungal pathogen leads to disease. Science advances 2(10):e1600822.

12. Faris JD, et al. (2010) A unique wheat disease resistance-like gene governs effector-triggered susceptibility to necrotrophic pathogens. Proceedings of the National Academy of Sciences 107(30):13544-13549.

13. Flor $\mathrm{HH}$ (1942) Inheritance of pathogenicity in Melampsora lini. Phytopathology 32:653-669.

14. Liu Z, et al. (2004) Genetic and physical mapping of a gene conditioning sensitivity in wheat to a partially purified host-selective toxin produced by Stagonospora nodorum. Phytopathology 94(10):1056-1060.

15. Liu Z, et al. (2012) The cysteine rich necrotrophic effector SnTox1 produced by Stagonospora nodorum triggers susceptibility of wheat lines harboring Snn1. PLOS Pathogens 8(1):e1002467.

16. Gijzen M \& Nürnberger T (2006) Nep1-like proteins from plant pathogens: Recruitment and diversification of the NPP1 domain across taxa. Phytochemistry 67(16):1800-1807.

17. Pemberton CL \& Salmond GP (2004) The NEP1-like proteins-a growing family of microbial elicitors of plant necrosis. Mol Plant Pathol 5(4):353-359

18. Bailey BA (1995) Purification of a protein from culture filtrates of Fusarium oxysporum that induces ethylene and necrosis in leaves of Erythroxylum coca. Phytopathology 85(10):1250-1255.

19. Fellbrich G, et al. (2002) Npp1, a phytophthora-associated trigger of plant defense in parsley and Arabidopsis. The Plant Journal 32(3):375-390.

20. Motteram J, et al. (2009) Molecular characterization and functional analysis of MgNlp, the sole NPP1 domaincontaining protein, from the fungal wheat leaf pathogen Mycosphaerella graminicola. Molecular Plant-Microbe Interactions 22(7):790-799.

21. Ben M'Barek S, et al. (2015) FPLC and liquid-chromatography mass spectrometry identify candidate necrosis-inducing proteins from culture filtrates of the fungal wheat pathogen Zymoseptoria tritici. Fungal Genet Biol 79:54-62.

22. Laugé R, et al. (1998) Successful search for a resistance gene in tomato targeted against a virulence factor of a fungal pathogen. Proceedings of the National Academy of Sciences Jul 95 (15) 9014-9018; DOI: 10.1073/ pnas.95.15.9014 pp 9014-9018.

23. Inohara N \& Nuñez G (2002) Ml - a conserved domain involved in innate immunity and lipid metabolism. Trends in Biochemical Sciences 27(5):219-221.

24. Mullen GED, et al. (2003) The role of disulfide bonds in the assembly and function of MD-2. Proceedings of the National Academy of Sciences 100(7):3919-3924.

25. Kettles GJ, et al. (2018) Characterization of an antimicrobial and phytotoxic ribonuclease secreted by the fungal wheat pathogen Zymoseptoria tritici. New Phytologist 217(1):320-331.

26. Daub ME \& Ehrenshaft M (2000) The photoactivated Cercospora toxin cercosporin: Contributions to plant disease and fundamental biology. Annual review of phytopathology 38(1):461-490.

27. Schlösser E (1962) Über eine biologisch aktive Substanz aus Cercospora beticola. Journal of Phytopathology 44(3):295-312.

28. Callahan TM, Rose MS, Meade MJ, Ehrenshaft M, \& Upchurch RG (1999) CFP, the putative cercosporin transporter of Cercospora kikuchii, is required for wild type cercosporin production, resistance, and virulence on soybean. Molecular plant-microbe interactions 12(10):901-910.

29. Choquer $M$, et al. (2005) The CTB1 gene encoding a fungal polyketide synthase is required for cercosporin biosynthesis and fungal virulence of Cercospora nicotianae. Molecular plant-microbe interactions 18(5):468-476. 
30. Kairys V, Gilson MK, \& Luy B (2004) Structural model for an AxxxG-mediated dimer of surfactant-associated protein C. European Journal of Biochemistry 271(11):2086-2092.

31. Bird LJ, Coleman ML, \& Newman DK (2013) Iron and copper act synergistically to delay anaerobic growth of bacteria. Applied and environmental microbiology 79(12):3619-3627.

32. Changela A, et al. (2003) Molecular basis of metal-ion selectivity and zeptomolar sensitivity by cuer. Science 301(5638):1383-1387.

33. Weltmeier F, et al. (2011) Transcript profiles in sugar beet genotypes uncover timing and strength of defense reactions to Cercospora beticola infection. Molecular Plant-Microbe Interactions 24(7):758-772.

34. Kim H, Ridenour JB, Dunkle LD, \& Bluhm BH (2011) Regulation of stomatal tropism and infection by light in Cercospora zeae-maydis: Evidence for coordinated host/pathogen responses to photoperiod? PLoS pathogens 7(7):e1002113.

35. Kami C, Lorrain S, Hornitschek P, \& Fankhauser C (2010) Chapter two - light-regulated plant growth and development. Current topics in developmental biology 91:29-66.

36. Roberts MR \& Paul ND (2006) Seduced by the dark side: Integrating molecular and ecological perspectives on the influence of light on plant defence against pests and pathogens. New Phytologist 170(4):677-699.

37. Roden LC \& Ingle RA (2009) Lights, rhythms, infection: The role of light and the circadian clock in determining the outcome of plant-pathogen interactions. The Plant Cell 21(9):2546-2552.

38. Guo A, Reimers PJ, \& Leach JE (1993) Effect of light on incompatible interactions between Xanthomonas oryzae pv oryzae and rice. Physiological and Molecular Plant Pathology 42(6):413-425.

39. Bailey BA, Dean JFD, \& Anderson JD (1990) An ethylene biosynthesis-inducing endoxylanase elicits electrolyte leakage and necrosis in Nicotiana tabacum cv xanthi leaves. Plant Physiology 94(4):1849-1854.

40. Brito N, Espino JJ, \& González C (2006) The endo- $\beta-1,4-X y l a n a s e ~ X y n 11 A$ is required for virulence in Botrytis cinerea. Molecular Plant-Microbe Interactions 19(1):25-32.

41. Kars I, et al. (2005) Necrotizing activity of five Botrytis cinerea endopolygalacturonases produced in Pichia pastoris. The Plant Journal 43(2):213-225.

42. Jalal MAF, Hossain MB, Robeson DJ, \& Van der Helm D (1992) Cercospora beticola phytotoxins: Cebetins that are photoactive, magnesium ion-binding, chlorinated anthraquinone-xanthone conjugates. J Am Chem Soc 114(15):5967-5971.

43. Yamazaki S, Okubo A, Akiyama Y, \& Fuwa K (1975) Cercosporin, a novel photodynamic pigment isolated from Cercospora kikuchii. Agricultural and Biological Chemistry 39(1):287-288.

44. Friesen TL \& Faris JD (2012) Characterization of plant-fungal interactions involving necrotrophic effectorproducing plant pathogens. Plant fungal pathogens: Methods and protocols, eds Bolton MD \& Thomma BPHJ (Humana Press, Totowa, NJ), pp 191-207.

45. Liu Z, et al. (2009) SnTox3 acts in effector triggered susceptibility to induce disease on wheat carrying the Snn3 gene. PLOS Pathogens 5(9):e1000581.

46. de Jonge R, et al. (2018) Gene cluster conservation provides insight into cercosporin biosynthesis and extends production to the genus Colletotrichum. Proceedings of the National Academy of Sciences, doi:10.1073/ pnas.1712798115.

47. Pfaffl MW (2001) A new mathematical model for relative quantification in real-time RT-PCR. Nucleic Acids Research 29(9):e45-e45.

48. Livak KJ \& Schmittgen TD (2001) Analysis of relative gene expression data using real-time quantitative PCR and the 2- $\triangle \Delta$ Ct method. Methods 25(4):402-408.

49. Bolton MD, et al. (2016) RNA-sequencing of Cercospora beticola DMI-sensitive and-resistant isolates after treatment with tetraconazole identifies common and contrasting pathway induction. Fungal Genetics and Biology 92:1-13.

50. Secor GA \& Rivera VV (2012) Fungicide resistance assays for fungal plant pathogens. Plant fungal pathogens: Methods and protocols:385-392. 


\section{Supplementary data}

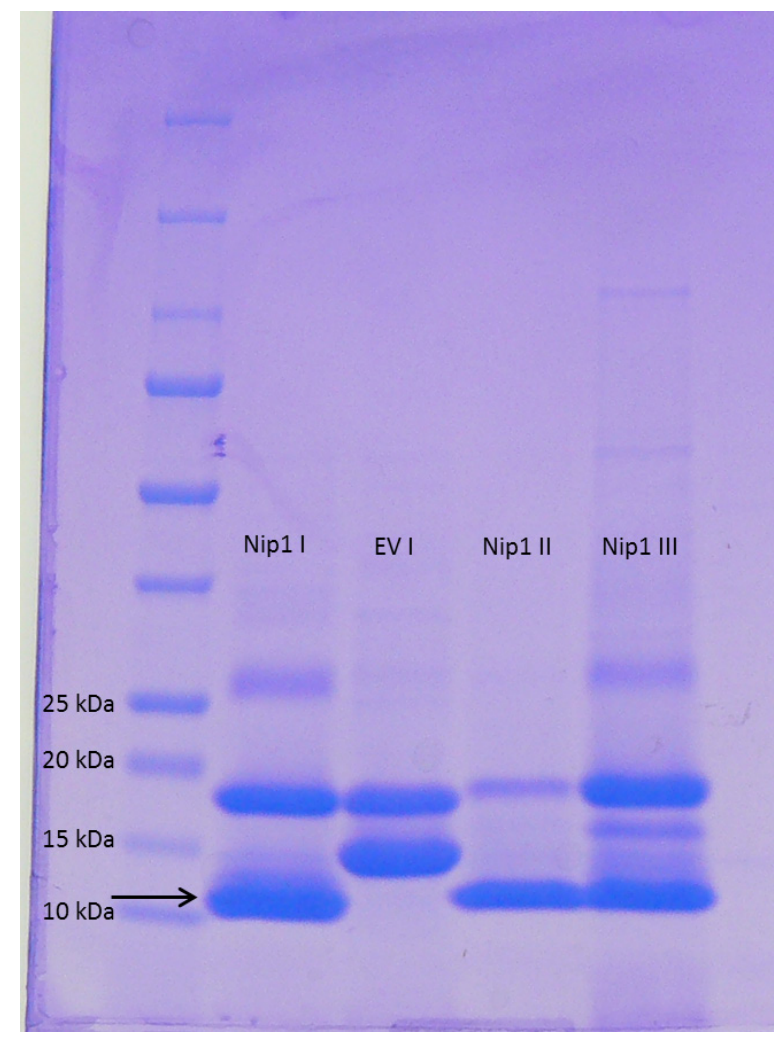

Supplementary Figure 1. Gel visualization of infiltrated CbNip1 protein and empty vector samples. SDSPAGE gel loaded with $5 \mu$ l of heterologously expressed protein sample used for infiltration studies. Nip1 I, Nip1 II, and Nip1 III are three individually produced and purified CbNip1 protein samples larrow indicates CbNip1 protein). EVI is the individually produced and purified empty vector sample used as control. 

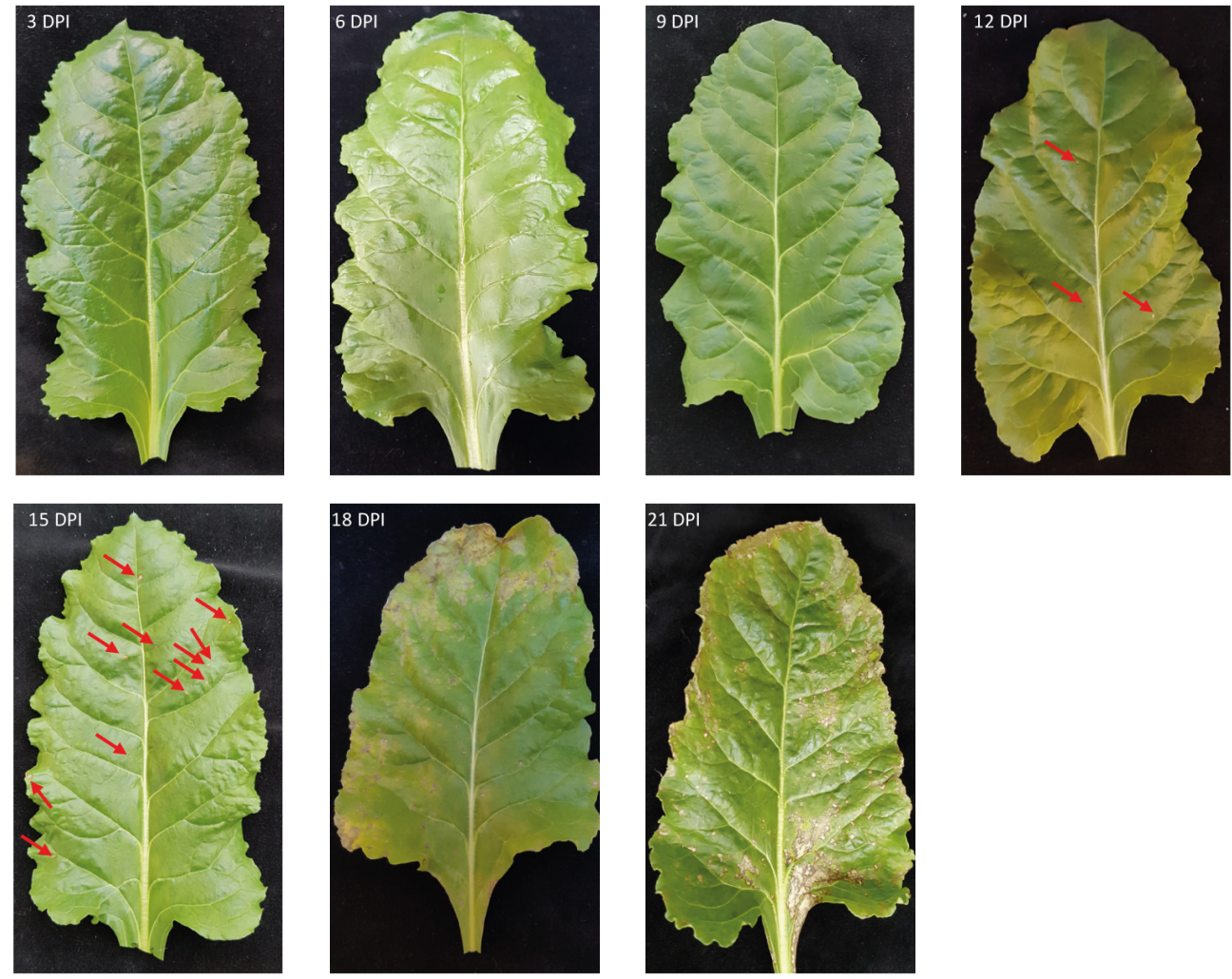

Supplementary Figure 2. Symptom development during C. beticola 09-40 wild type strain infection of sugar beet. No symptoms are detectable at early time points (3, 6, and 9 DPI). Red arrows at 12 and 15 DPI indicate formation of necrotic lesions. At $18 \mathrm{DPI}$, necrotic lesion formation intensifies, accompanied by some chlorosis around the necrotic lesions. At $21 \mathrm{DPI}$, single necrotic lesions are fused to form necrotic patches on the sugar beet leaf. 
Table S1. Primers used in this study.

\begin{tabular}{|c|c|c|}
\hline Primer & Sequence $\left(5^{\prime}-3^{\prime}\right)$ & Description \\
\hline MKE-78 & $\begin{array}{l}C \quad G \quad G \quad T \quad A \quad T \quad G \quad A \quad A \quad T \quad T \quad C \\
\text { GGCAAAGACCACTCCGAGCAC }\end{array}$ & CbNip1 Fp with EcoRI RE site for insertion into $p E T$ \\
\hline MKE-77 & $\begin{array}{l}\text { CGTCTAGCGGCCGCCTACTACTA } \\
\text { GCAAGTTCCACGGTAACCCGC }\end{array}$ & $\begin{array}{l}\text { CbNip1 Rp with triple stop codon and Notl RE site for } \\
\text { insertion into } \mathrm{pET}\end{array}$ \\
\hline MDB-726 & ACTTGCCTGGCTTTTGTTTCTAGT & SbEc1-F qPCR for sugar beet biomass \\
\hline MDB-727 & GCCAGGTGCTGACTTGATTATTT & SbEc1-R qPCR for sugar beet biomass \\
\hline MDB-284 & ACATGGCTGGTCGTGATTTG & C.beticola actin qPCR Fp \\
\hline MDB-285 & TGTCCGTCAGGAAGCTCGTA & C.beticola actin qPCR Rp \\
\hline MDB-1063 & AGACCACTCCGAGCACAACT & CbNip1 qPCR Fp \\
\hline MDB-1064 & ACACCGTTGTCGTAGGTTCC & CbNip1 qPCR Rp \\
\hline MDB-957 & CCTGTGGTCTGAGCTTGTCA & CbNip1 1F for KO \\
\hline MDB-958 & $\begin{array}{l}\text { CACTGGCCGTCGTTTTACAACGTC } \\
\text { TCCAACTGTTCTCCCTGTCC }\end{array}$ & CbNip1 2R for KO \\
\hline MDB-959 & $\begin{array}{l}\text { TCATGGTCATAGCTGTTTCCTGTG } \\
\text { GGTTGTTGGGGAGTTTCTGA }\end{array}$ & CbNip1 3F for KO \\
\hline MDB-960 & CACCACTTGGTATCGGGAAC & CbNip1 4R for KO \\
\hline MDB-1541 & AGCCGCTAATCACCCAAGAT & CbNip1 5p1F \\
\hline MDB-277 & GACGTTGTAAAACGACGGCCAGTG & Split-marker HYG-F \\
\hline MDB-258 & GGATGCCTCCGCTCGAAGTA & Split-marker HY-R \\
\hline MDB-259 & CGTTGCAAGACCTGCCTGAA & Split-marker YG-F \\
\hline MDB-278 & CACAGGAAACAGCTATGACCATGA & Split-marker HYG-R \\
\hline MDB-1145 & GGCAGGTAGATGACGACCAT & HYG R2 \\
\hline
\end{tabular}





\section{Gene cluster conservation provides insight into cercosporin biosynthesis and extends production to the genus Colletotrichum}

Ronnie de Jonge*,\#, Malaika K. Ebert*, Callie R. Huitt-Roehl*,

Paramita Pal, Jeffrey C. Suttle, Rebecca E. Spanner,

Jonathan D. Neubauer, Wayne M. Jurick II, Karina A. Stott, Gary A. Secor, Bart P. H. J. Thomma, Yves Van de Peer, Craig A. Townsend\#, and Melvin D. Bolton\# (2018) Gene cluster conservation provides insight into cercosporin biosynthesis and extends production to the genus Colletotrichum. Proceedings of the National Academy of Sciences 115, 5459 - 5466; doi: https://doi.org/10.1073/pnas.1712798115

* These authors contributed equally

\# These authors contributed equally 


\begin{abstract}
Species in the genus Cercospora cause economically devastating diseases in sugar beet, maize, rice, soy bean and other major food crops. Here we sequenced the genome of the sugar beet pathogen $C$. beticola and found it encodes 63 putative secondary metabolite gene clusters, including the cercosporin toxin biosynthesis (CTB) cluster. We show that the CTB gene cluster has experienced multiple duplications and horizontal transfers across a spectrum of plant pathogenic fungi, including the wide-host range Colletotrichum genus as well as the rice pathogen Magnaporthe oryzae. Although cercosporin biosynthesis has been thought todate to rely on an eight gene CTB cluster, our phylogenomic analysis revealed gene collinearity adjacent to the established cluster in all CTB cluster-harboring species. We demonstrate that the CTB cluster is larger than previously recognized and includes cercosporin facilitator protein (CFP), previously shown to be involved with cercosporin auto-resistance, and four additional genes required for cercosporin biosynthesis, including the final pathway enzymes that install the unusual cercosporin methylenedioxy bridge. Finally, we demonstrate production of cercosporin by Colletotrichum fioriniae, the first known cercosporin producer within this agriculturally important genus. Thus, our results provide new insight into the intricate evolution and biology of a toxin critical to agriculture and broaden the production of cercosporin to another fungal genus containing many plant pathogens of important crops worldwide.
\end{abstract}




\section{Introduction}

Cercospora are among the most speciose genera in all Fungi (1). First described in 1863 (2), the genus has sustained a long history, largely due to notoriety as the causal agent of leaf spot diseases in a wide range of plants including agriculturally important crops such as sugar beet, soybean, maize, and rice that together account for hundreds of millions of dollars in lost revenue annually to growers worldwide (3-8). Although Cercospora spp. share several characteristics associated with pathogenicity, such as penetration through natural openings and extracellular growth during the biotrophic stage of infection, most rely on the production of the secondary metabolite (SM) cercosporin (1) to facilitate infection $(9,10)$. Studies spanning nearly 60 years have made cercosporin a model perylenequinone (11), a class of SMs characterized by a core pentacyclic conjugated chromophore that gives rise to its photoactivity. When exposed to ambient light, cercosporin is a potent producer of reactive oxygen species in the presence of oxygen (12) with a quantum efficiency of $>80 \%$ (13). This small molecule is lipophilic and can readily penetrate plant leaves leading to indiscriminate cellular damage within minutes of exposure (14). Indeed, cercosporin is nearly universally toxic to a wide array of organisms including bacteria, mammals, plants, and most fungal species with the key exception of cercosporinproducing fungi, which exhibit cercosporin auto-resistance. To date, cercosporin has only been reported to be produced by Cercospora spp., with the single exception of the brassica pathogen Pseudocercosporella capsellae (15). However, Pseudocercosporella and Cercospora are phylogenetically closely related, residing in a large clade within the Mycosphaerellaceae (16).

In contrast to the large body of information on cercosporin biology spanning several decades $(17,18)$, the cercosporin toxin biosynthesis (CTB) gene cluster was only recently resolved in C. nicotianae (19). The keystone enzyme for cercosporin biosynthesis, CTB1, bears all the hallmarks of an iterative, non-reducing polyketide synthase (NR-PKS) (20). Using CTB1 as a point of reference, the complete C. nicotianae CTB gene cluster was determined to consist of eight contiguous genes of which six are believed to be responsible for cercosporin assembly (CTB1, 2, 3, 5, 6, and 7) (19, 21). The zinc finger transcription factor CTB8 co-regulates expression of the cluster (19), while the major facilitator superfamily (MFS) transporter CTB4 exports the final metabolite (22). Downstream of the CTB cluster are two open reading frames (ORFs) encoding truncated transcription factors, while loci designated as ORF9 and ORF10 upstream of the CTB cluster are not regulated by light and are not believed to encode proteins with metabolic functions (19). Consequently, the clustering of eight genes with demonstrated co-regulation by light that are flanked by ORFs with no apparent role in cercosporin biosynthesis has suggested that cercosporin production relies on the eight-gene CTB cluster (19). In this study, we used an evolutionary comparative genomics approach to show that the CTB gene cluster underwent multiple duplication events and was transferred horizontally across large taxonomic distances. Since these horizontal transfer events included genes adjacent to the canonical eight gene CTB cluster, we used reverse genetics to show that the CTB cluster includes additional genes in C. beticola, including one gene that was previously shown to be involved with cercosporin auto-resistance (23) and four previously unrecognized genes involved with biosynthesis. The CTB cluster was found in several Colletotrichum (Co.) species, and we confirmed that the apple pathogen Co. fioriniae 
can also produce cercosporin. As all earlier understanding of cercosporin biosynthesis has been unwittingly limited by a truncated set of genes in Cercospora spp., the full dimension of the gene cluster provides deeper insight into the evolution, biosynthesis and dissemination of a fungal toxin critical to world-wide agriculture.

\section{Results}

\section{Secondary metabolite cluster expansion in Cercospora beticola.}

C. beticola strain 09-40 was sequenced to 100-fold coverage and scaffolded with optical and genome maps, resulting in $96.5 \%$ of the $37.06 \mathrm{Mbp}$ assembly being placed in 12 supercontigs of which 10 are assumed to be chromosomes. Despite their ubiquitous presence in nature and cropping systems, genome sequences of Cercospora spp. are not well-represented in public databases. Therefore, to aid comparative analysis within the Cercospora genus we also sequenced the genome of C. berteroae and reassembled the genome of C. canescens (24) (Suppl. Table S1). To identify gene clusters responsible for biosynthesis of aromatic polyketides in C. beticola, we mined the genome to identify all SM clusters (25) and compared these with predicted clusters in related Dothideomycete fungi. The C. beticola genome possesses a total of 63 predicted SM clusters of several classes, representing an expanded SM repertoire with almost twice the number when compared to closely related Dothideomycetes, which average 34 SM clusters (Suppl. Table S2; Dataset S1). Notably, C. beticola encodes 23 candidate non-ribosomal peptide synthetase (NRPS) clusters, which is considerably higher than most Dothideomycetes, which have an average of 13 (26). To identify the C. beticola PKS cluster responsible for cercosporin biosynthesis, we compared the sequence of the C. nicotianae CTB cluster (19) with predicted PKS clusters of $C$. beticola. To fill in sequencing gaps between genes in the $C$. nicotianae CTB cluster, we sequenced the genome of C. nicotianae, which showed that C. beticola PKS CBET3_00833 (CbCTB1) and flanking genes (CBET3_00830 - CBET3_00837) were 96\% identical to C. nicotianae CTB1 - CTB8 and all genes were collinear, strongly suggesting this region houses the CTB cluster in C. beticola (Suppl. Fig. S1).

\section{Repeated duplication and lateral transfer of the cercosporin biosynthetic cluster.}

To study the evolutionary relationships of C. beticola PKSs, we conducted large-scale phylogenomic analyses that included various previously characterized PKSS from selected species (27). Since resolving orthologous relationships among PKSs can predict the type of SM that will be synthesized, we first built a phylogenetic tree of the conserved core $\beta$-ketoacyl synthase (KS) domains of each PKS that resulted in separating PKS enzymes into four major groups (Suppl. Fig. S2A). Among the eight C. beticola NR-PKSs, phylogenetic analysis revealed significant similarity between CbCTB1, CBET3_10910-RA, and CBET3_11350-RA which cluster at the base of the cercosporin clade (Suppl. Fig. S2B). Interestingly, genes flanking CBET3_10910-RA, but not CBET3_11350-RA, were also strikingly similar to CbCTB cluster genes (Fig. 1). Consequently, we hypothesize that the CBET3_10910 SM cluster is the result of a CTB cluster duplication. Since duplicated SM gene clusters appeared to be relatively rare in fungi (28), we investigated the origin and specificity of the CTB cluster and the putative duplication by searching for CbCTB1 homologs against a selected set of 48 published Ascomycete proteomes (Suppl. Table S3) representing a 
diverse group of fungal orders. We identified CbCTB1 orthologs in Cercospora spp. C. berteroae and C. canescens and confirmed its presence in Cladosporium fulvum (27) and Parastagonospora nodorum (29). Surprisingly, seven additional orthologs were identified in Sordariomycete species Co. orbiculare, Co. gloeosporioides, Co. fioriniae, Co. graminicola, Co. higginsianum, and Magnaporthe oryzae as well as one in the Leotiomycete Sclerotinia sclerotiorum (Suppl. Fig. S3A), representing diverse taxa harboring CTB1. Analysis of sequence identity showed that intra-species (CbCTB1 - CBET3_10910-RA) sequence identity (45\%) was lower than the inter-species identity (e.g. CbCTB1 and C. fulvum CTB1 (Clafu1_196875) sequence identity is 55\%; Suppl. Table S4), suggesting that the CTB1 duplication event occurred prior to Dothideomycete speciation.

To develop a 'phylogenetic roadmap' that may explain CTB1 evolution, we used the process of 'reconciliation' that takes into account both species and gene histories (30). Although not conclusive, reconciliation considers the costs of evolutionary events (i.e. gene duplications, transfers, and/ or losses) to explain the most parsimonious evolutionary route to the present scenario (31). Reconciliation of the species tree (Suppl. Fig. S4) with the CTB1 protein tree revealed that the predicted evolutionary history of CTB1 can be characterized by four duplications, three transfers, and widespread loss to most species analyzed (Suppl. Fig. S5A), and further corroborates our hypothesis that the CTB1 duplication event (D1) occurred early in Dothideomycete speciation. Reconciliation also revealed an ancient CTB1 ortholog in S. sclerotiorum (Suppl. Fig. S5A), suggesting that CTB1 arose prior to speciation of Dothideomycetes. Duplications 2-4 (D2-4) arose after lateral transfer (T1) of CTB1 into the last common ancestor of the Glomerellales. CTB1 was then transferred (T2) from a common ancestor in the Glomerellales to M. oryzae (Suppl. Fig. S5A).

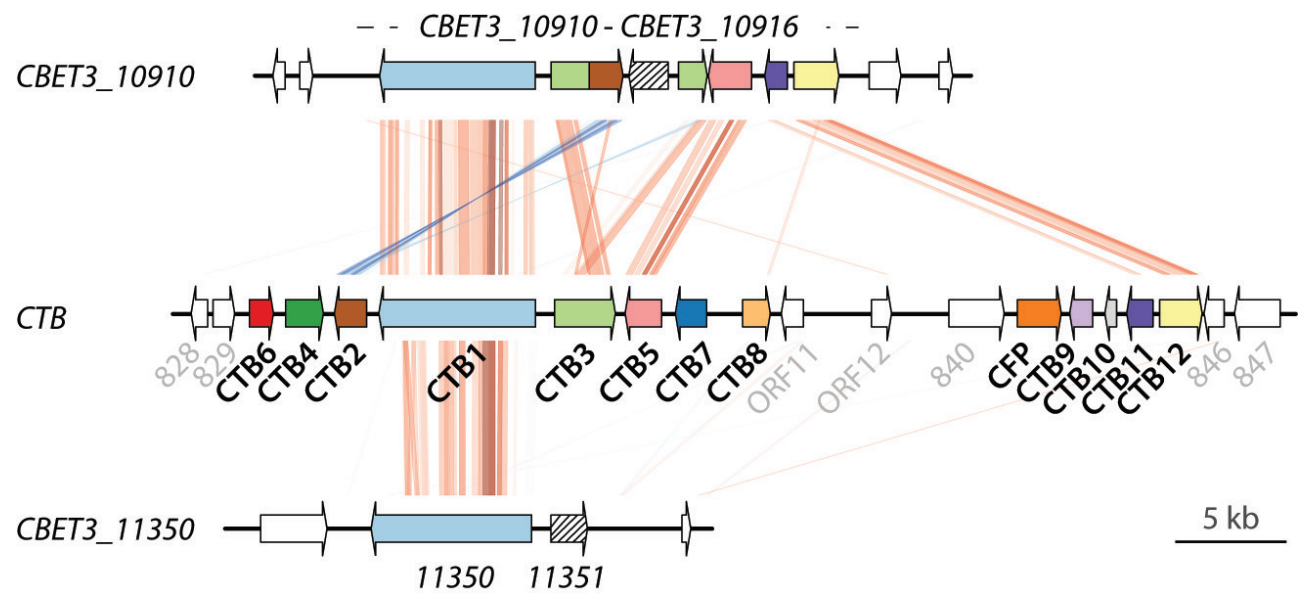

Figure 1. The cercosporin biosynthetic cluster is duplicated and maintained in C. beticola. CBET3_10910 and flanking genes are syntenic with the CTB cluster (CBET3_00833 and flanking genes) in C. beticola. Alignment lines correspond to DNA fragments exhibiting significant similarity when the genomic regions comprising the gene clusters are compared with tBLASTx. Direct hits are displayed in red, whereas complementary hits are in blue. The intensity of the alignments represents the percentage similarity ranging from 23 to 100 percent. Genes flanking CBET3_11350-RA were not syntenic with CTB cluster genes. 
We extended the search for CTB cluster protein orthologs by scanning the 48 proteomes for homologs of CbCTB2 (CBET3_00830) to CbCTB8 (CBET3_00837) followed by phylogenetic tree construction and subtree selection (Suppl. Fig. S3B-N). This resulted in the identification of orthologs in the same set of species previously listed to contain CTB1, with the only exceptions in cases where CTB gene homologs were lost in a species. Although the loss of CTB6 and CTB7 orthologs limits reconciliation analysis of these gene families, reconciliation of the subtrees for CTB2, CTB3, CTB4, CTB5, and CTB8 (Suppl. Fig. S5B-H) supported a similar scenario as proposed for CTB1, involving at least two duplications (D1 and D2) and two horizontal transfer events (T1 and T2) that explain the present-day CTB scenario (Fig. 2). However, an alternative explanation involving a single transfer to an ancestral Glomerellales species followed by widespread loss in most species in this lineage except for M. oryzae and the analyzed Colletotrichum spp. (Fig. 2; Suppl. Table S5) cannot be ruled out by our analyses at this stage.

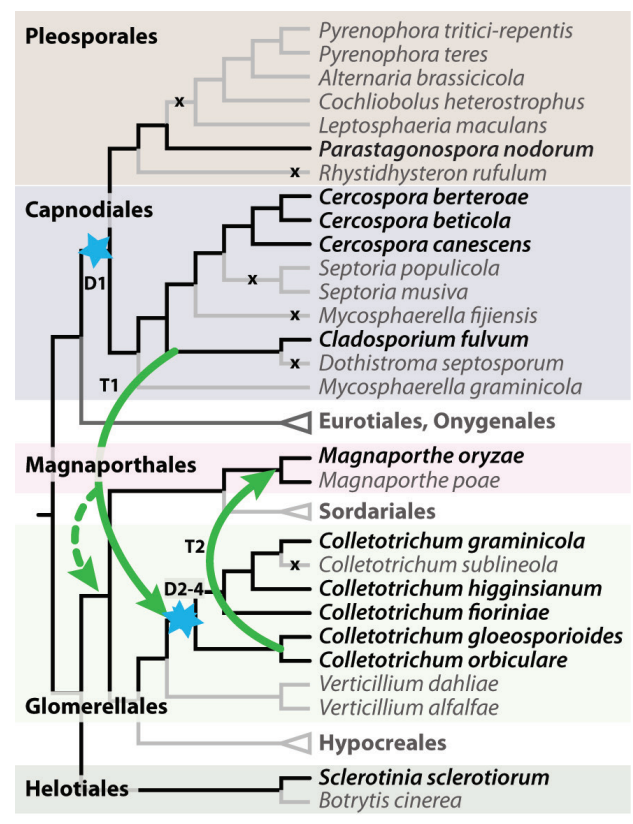

Figure 2. Phylogenetic roadmap of СТВ cluster evolution. Phylogenetic roadmap detailing the proposed evolutionary trajectory of the CTB cluster involving horizontal gene transfer events from the Capnodiales to the Glomerellales (T1) and another from the Glomerellales to the Magnaporthales (T2) as well as multiple duplications (D1-4) and frequent gene loss $(x)$. Cladogram of the phylogenetic relationship of Cercospora spp. and 45 other sequenced fungi. The unscaled tree was constructed using CVTree. Duplication nodes are marked with blue stars, losses are indicated by the crosses and transfers are highlighted by green arrows. Species without the CTB cluster are depicted in grey, those encompassing it are in black. An alternative and slightly less parsimonious scenario involving a single transfer from Capnodiales into the last common ancestor of the Magnaporthales and the Glomerellales is shown by the dashed arrow.

\section{Extension of the predicted cercosporin biosynthetic cluster based on microsynteny.}

To further examine the CTB clusters across all recipient species we generated pairwise alignments relative to the C. beticola CTB cluster and flanks. To our surprise, we observed a striking level of similarity outside of the known eight CTB genes on the 3 ' end of the cluster (Fig. 3) in all CTB-containing genomes. To investigate whether the amount of microsynteny observed for CTB cluster and these flanking genes can be reasonably expected when comparing Dothideomycete and Sordariomycete genomes, we assessed the genome-wide microsynteny between the genomes of C. beticola and Co. gloeosporioides and C. beticola and M. oryzae. This analysis identified the CTB cluster together with its flanking genes as having 
the highest level of microsynteny among all regions in the genome between C. beticola and Co. gloeosporioides, and showed that the observed CTB microsynteny between C. beticola and M. oryzae was also higher than the genome-wide average (Fig. 4). Likewise, sequence identity of most CTB proteins between C. beticola and Colletotrichum spp., and to a lesser degree with M. oryzae, is higher compared to the genome-wide average (Suppl. Fig. S6, S7). In contrast, sequence conservation of CTB8, a $\mathrm{Zn}_{2} \mathrm{Cys}_{6}$ transcription factor previously implicated for transcriptional regulation of the CTB cluster (19), appears much lower than that of other CTB- and non-CTB proteins, and therefore is suggestive of positive diversifying selection.

C. beticola

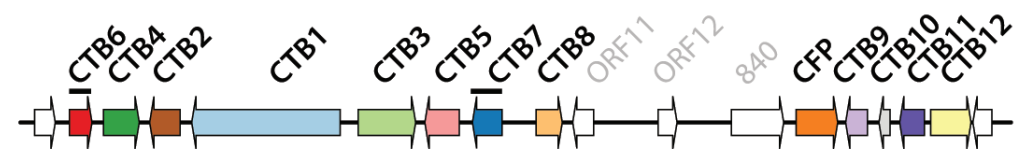

- - CBET3_00830-CBET3_00845

C. fulvum

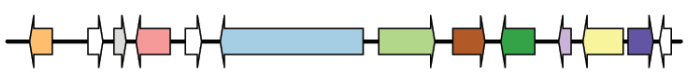

$----196962-196951$

Co. higginsianum

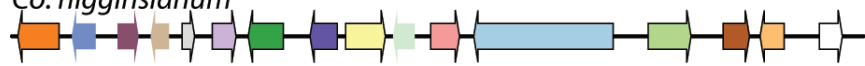

$-\ldots+\ldots$ - $-\ldots$ CH63R_06234-CH063R_06248 - -

Co. graminicola

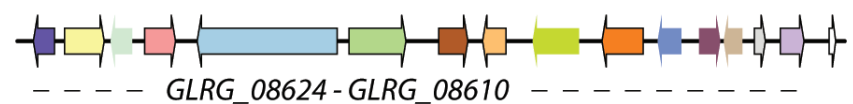

M. oryzae

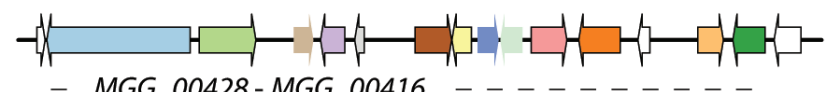

- MGG_00428-MGG_00416 _ . - . - . -

P. nodorum

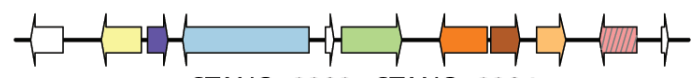

- - STANO_2392-STANO_2384 - - - -

\section{Polyketide synthase}

O-methyltransferase

$\square$ O-methyltransferase/FAD-dependent monooxygenase

Major Facilitator Superfamily (MFS) transporter

$\square$ Oxygen-, FAD/FMN-dependent oxidoreductase

NADPH-dependent oxidoreductase

FAD/FMN-dependent oxidoreductase

Zinc-finger transcription factor

MFS transporter
GA4 destaturase family protein

Beta-ig-h3 fasciclin

Laccase

Dehydratase

Short-chain dehydrogenase

GA4 desaturase

Short-chain dehydrogenase

NmrA-like family protein

Figure 3. Synteny and rearrangements of the conserved $C$. beticola cercosporin biosynthetic cluster. The cercosporin biosynthetic cluster in C. beticola (Cb), top line, and flanking genes are conserved in Cladosporium fulvum, Co. higginsianum, Co. graminicola, M. oryzae and Parastagonospora nodorum. For all species, the displayed identifiers are transcript IDs and the corresponding sequences can be retrieved from JGI MycoCosm or ORCAE. CTB orthologs are colored relative to the $C$. beticola CTB cluster genes and the color key, as well as annotated functions, are highlighted below the CTB cluster graphic. Cercospora-specific CTB genes CTB6 and CTB7 are underlined. 


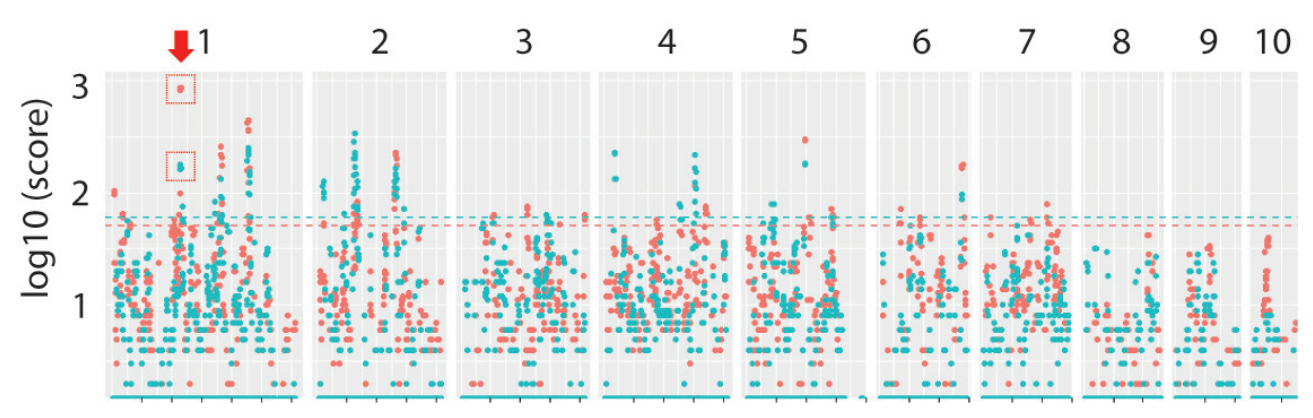

Figure 4. СTB cluster microsynteny conservation segregates from the genome-wide average. The genomewide, gene-by-gene microsynteny between Cercospora beticola and Colletotrichum gloeosporioides ICg, red), and between $C$. beticola and M. oryzae (Mo, blue), across the ten assembled C. beticola chromosomes is shown. Each dot represents one $C$. beticola gene and its respective microsynteny score. The red arrow indicates the position of the CTB cluster on chromosome 1 and coincides with high microsynteny in both Co. gloeosporioides and M. oryzae. The dashed lines represent the $99^{\text {th }}$ quantile of the microsynteny scores for both comparisons independently.

flanking genes are part of the C. beticola CTB cluster. To test this proposal, we first determined the relative expression of all eight established $C$. beticola CTB genes as well as a number of flanking genes (CBET3_00828 to CBET3_00848) under light (cercosporin-inducing) compared to dark (cercosporin-repressing) conditions, which showed that all candidate CTB genes on the 3' flank were induced in the light except CBET3_00846 and CBET3_00848 (Suppl. Table S7). Functional annotation of these genes revealed one non-conserved phenylalanine ammonia lyase (CBET3_00840), the cercosporin facilitator protein (CFP) (23) (CBET3_00841), a candidate a-ketoglutarate-dependent dioxygenase (CBET3_00842), a dehydratase (CBET3_00843), a $\beta$-ig-h3 fasciclin (CBET3_00844), a laccase (CBET3_00845), zinc finger domain-containing protein (CBET3_00846), and protein phosphatase 2A (CBET3_00847; Suppl. Table S7), several of which have functions associated with multi-domain enzymes or polyketide biosynthesis in fungi or bacteria $(19,32-37)$. Phylogenetic analyses of these flanking genes and reconciliation of their respective protein phylogenies (Suppl. Fig. S3, S5) with the species tree (Suppl. Fig. S4) suggest that all genes except CBET3_00840, CBET3_00846, and CBET3_00847 have undergone highly similar evolutionary trajectories as the established CTB cluster genes (Fig. 2. Suppl. Fig. S5) suggesting that the CTB cluster was transferred as a whole at least once, followed by species-specific evolutionary trajectories involving frequent gene loss as well as gene gain (Fig. 2). We further evaluated the hypothesis of horizontal cluster transfer using a comparative topology test that examines whether the determined tree topologies that support horizontal cluster transfer are significantly better than constrained topologies that would not support transfer. Tree topologies were compared using the Approximately Unbiased test (38), implemented in CONSEL (39) as previously described by Wisecaver and Rokas (2015) (40). Constrained topologies in which we force either a monophyletic origin of all Dothideomycete proteins or a monophyletic origin of all Sordariomycete proteins were significantly worse than trees without such constraint (Suppl. Table S6). Thus, the comparative topology tests support the previously determined topologies, which suggest horizontal cluster transfer. 


\section{Novel CTB genes are essential for cercosporin biosynthesis.}

To confirm individual gene contributions for cercosporin production, we generated single gene deletion mutants of all candidate genes from CBET3_00840 to CBET3_00846 and tested their ability to produce cercosporin. Initial assays of selected mutants showed that cercosporin production in $\triangle C B E T 3 \_00844$ and $\triangle C B E T 3 \_00845$ mutants was abolished, while $\triangle C B E T 3$ _00842 mutants accumulated only a red, cercosporin-like metabolite that migrated differently in potato dextrose agar (PDA) culture plates and thin layer chromatography (TLC) (Suppl. Fig. S8). To provide more definitive analyses of cercosporin production, high-performance liquid chromatography (HPLC) profiles were obtained from all candidate CTB gene mutants and compared to WT cercosporin (Fig. 5A). Unlike other analyzed mutants, $\triangle C B E T 3 \_00840$ and $\triangle C B E T 3 \_00846$ produced compounds with HPLC profiles like cercosporin (Fig. 5A), suggesting these genes are not involved with cercosporin biosynthesis. Taken together, these results corroborate our hypothesis that the CTB
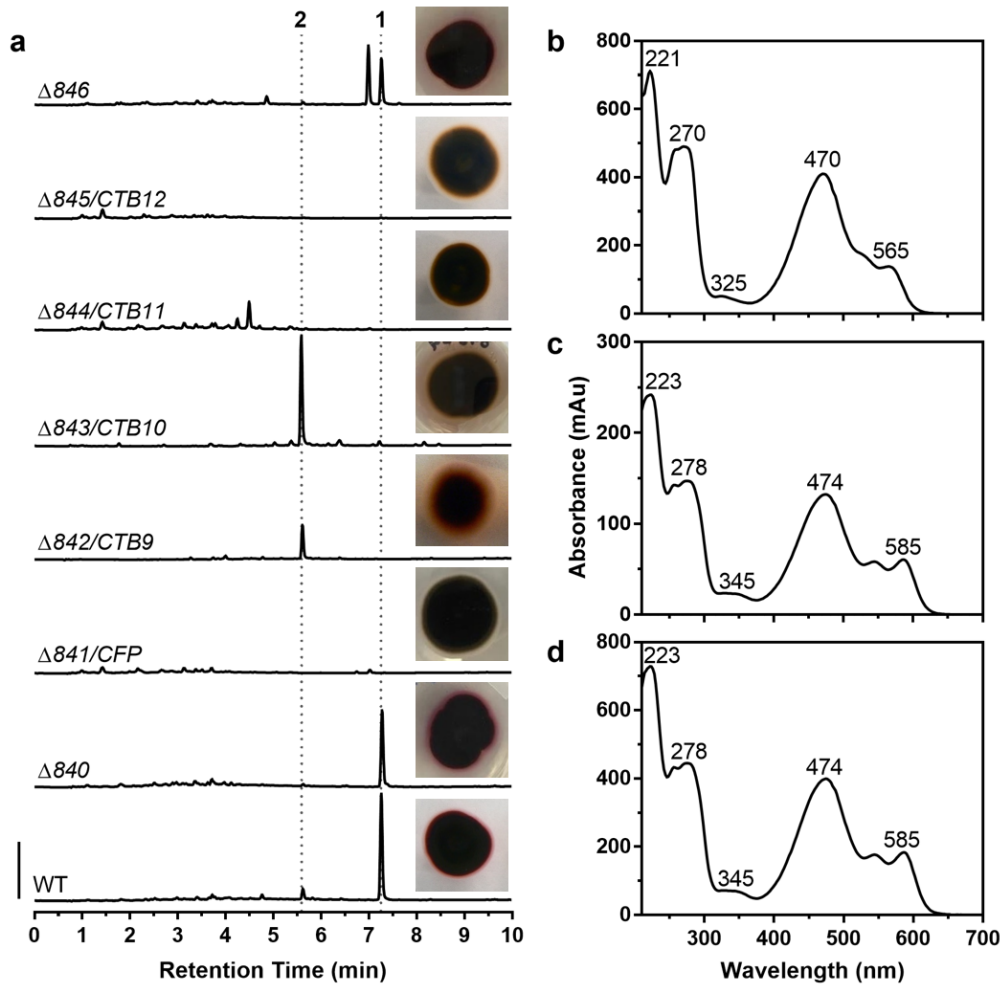

Figure 5. Analysis of cercosporin production in CTB mutants of $\boldsymbol{C}$. beticola. Site-directed knock-out mutants in genes CBET3_00840,CFP (CBET3_00841), CTB9 (CBET3_00842), CTB10 (CBET3_00843), CTB11 (CBET3_00844), CTB12 (CBET3_00845) and CBET3_00846 were assayed for cercosporin production by HPLC. Cercosporin extracted from C. beticola strain 10-73-4 (WT) was used as a positive control. a) $280 \mathrm{~nm}$ HPLC chromatograms and images of representative colonies for each knock-out. Scale bar indicates $250 \mathrm{mAu}$. Cercosporin (1) and pre-cercosporin (2) peaks are indicated by dashed lines. b-d) UV-Vis spectra from wild-type C. beticola (b, 7.25 min peak), C. beticola $\triangle C T B 9$ (c, $5.36 \mathrm{~min}$ peak), and C. beticola $\triangle C T B 10$ (d, $5.36 \mathrm{~min}$ peak) were extracted from $280 \mathrm{~nm}$ HPLC chromatograms. Wavelengths of relevant UV maxima are indicated. 
cluster extends to at least CBET3_00845 at the 3' side and includes four additional CTB biosynthetic genes as well as CbCFP. Consequently, we propose naming genes CBET3_00842, CBET3_00843, CBET3_00844 and CBET_00845 as CTB9 to CTB12, respectively (Suppl. Table S7).

\section{Pre-cercosporin isolation and characterization.}

To characterize the red metabolite that accumulated in the $\triangle 842 / C T B 9$ and $\triangle 843 /$ CTB10 mutants (Fig. 5A; (Suppl. Fig. S8), an ethyl acetate extract of the collected mycelia was analyzed by reverse-phase HPLC. At $280 \mathrm{~nm}$, a single peak was observed in both mutant extracts with identical retention time and UV-Vis spectra (Fig. 5). This peak was compared to a reference sample of cercosporin produced by wild-type C. beticola. The retention time of this peak was shorter than that of cercosporin suggesting a more polar metabolite. Comparison of the UV-vis spectra (Fig. 5B-D) of the unknown compound and cercosporin revealed nearly identical chromophores, suggesting close structural relation. The exact mass of the metabolite from the mutants was determined ( $\triangle 842 / C T B 9: \mathrm{m} / \mathrm{z}=$ 537.1762, $\triangle 843 / C T B 10: \mathrm{m} / \mathrm{z}=537.1757,[\mathrm{M}+\mathrm{H}+])$, consistent with the elemental composition $\mathrm{C}_{29} \mathrm{H}_{28} \mathrm{O}_{10}$. This mass is $2 \mathrm{Da}$ greater than that of cercosporin (+2 hydrogens), which led to a proposed structure for pre-cercosporin (2) (Fig. 6). Alternative hydroquinones of cercosporin could be excluded simply on the basis of the UV-vis spectral information and chemical instability. The presence of a free phenol in pre-cercosporin in place of the unusual 7-membered methylenedioxy of cercosporin is consonant with the red shift of the long wavelength $\lambda_{\max }$ and the shorter HPLC retention time. To firmly support the tentative structure of pre-cercosporin, the crude extract of $\triangle 842 / C T B 9$ was further purified by reverse-phase HPLC. To obtain sufficient material for ${ }^{1} \mathrm{H}-\mathrm{NMR}$ analysis, extractions were performed quickly and in low light and reduced temperature to slow apparentpolymerization of pre-cercosporin. The relative instability of precercosporin compared to cercosporin suggests a possible role for the methylenedioxy bridge in overall stability. Immediately evident in the ${ }^{1} \mathrm{H}-\mathrm{NMR}$ spectrum (Suppl. Fig. S9A), apart from its
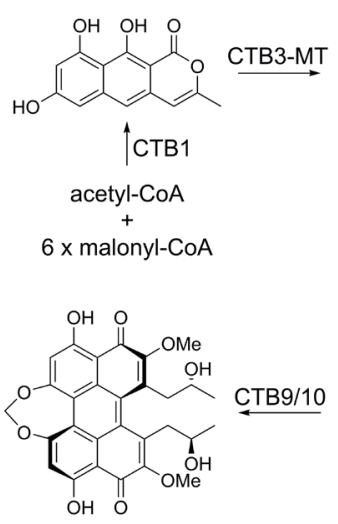

cercosporin 1

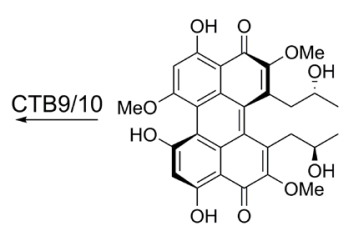

pre-cercosporin 2

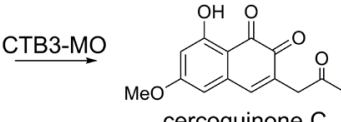

cercoquinone $\mathrm{C}$

Dimerization (CTB11/12?)

Methylation (CTB2)

Reduction (CTB6?)

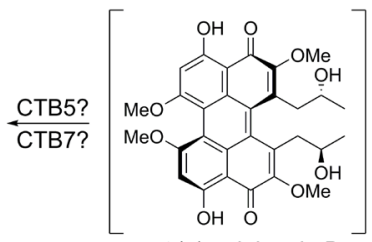

ent (+)-calphostin D

Figure 6. Proposed biogenesis of cercosporin. Tentative proposal for biosynthesis of cercosporin (1), incorporating newly discovered biosynthetic genes. Intermediates in brackets are logically inferred, and have not been directly observed. MT = methyltransferase, $M O=$ monooxygenase. 
overall similarity to that of cercosporin itself, was the absence of the methylenedioxy singlet at $\delta 5.74$ diagnostic of cercosporin, but the appearance of a new methoxyl signal at $\delta 4.28$ and a phenol at 89.25 . Consistent with the new asymmetry in pre-cercosporin, two strongly hydrogenbonded peri-hydroxy groups could be seen far downfield at ca. 15 ppm and two aryl hydrogens were observed at $\delta 6.92$ and $\delta 6.87$. That these latter resonances are observed only in pairs, as are the two side chain methyl doublets at ca. 0.6 ppm, and the doubling of other signals imply that pre-cercosporin is formed as a single atropisomer having a helical configuration likely identical to that of cercosporin, although it is conceivable CTB9 or CTB10 sets the final stereochemistry.

${ }^{13} \mathrm{C}-\mathrm{NMR}$ data were obtained by growing a larger number of PDA plates of $\triangle 842 / C T B 9$ supplemented with $2 \mathrm{mM}$ each $\left[1-{ }^{13} \mathrm{C}\right]$ - and $\left[2-{ }^{13} \mathrm{C}\right]$-sodium acetate to equally enrich all polyketidederived carbons ( $3 \% / s i t e)$. Working quickly to isolate and purify pre-cercosporin as above in low light and low temperature, both 1D and HSQC spectra of pre-cercosporin were acquired (Suppl. Fig. S9B and S9C). As seen in the ${ }^{1} \mathrm{H}-\mathrm{NMR}$ spectrum, breaking the symmetry of cercosporin was evident in the observation of all 29 carbons in the ${ }^{13} \mathrm{C}-\mathrm{NMR}$ spectrum, which notably revealed three methoxyl groups and diagnostic doubling of all resonances, save two overlapping pairs of signals. This behavior is fully in accord with the assigned structure of pre-cercosporin.

\section{Identification of cercosporin from Co. fioriniae.}

Since our initial phylogenomic analyses suggested that several Colletotrichum spp. harbored CTB clusters (Figs. 2, 3), we questioned whether the CTB cluster can be found in additional Colletotrichum spp. CTB protein orthology analysis revealed that eight out of the 13 Colletotrichum spp. hosted at Ensemble Fungi (https://fungi.ensembl.org/index.html) encode a similar set of CTB proteins as observed in Co. higginsianum (Suppl. Table S8). These eight species are plant pathogens of crops such as apple, safflower, melon, cucumber, and a variety of Brassica and cereal crops, as well as various tree species (41-47) (Suppl. Table S8, Figs. S10, S11). Remarkably, many species have lost several CTB genes such as the endophyte Co. tofieldiae, which has lost the cluster entirely (Suppl. Table S8, Figs. S10, S11).

Since earlier reports suggested the production of a "red pigment" by some Colletotrichum spp. such as the apple pathogen Co. fioriniae $(48,49)$, we questioned whether the red pigment was cercosporin. As a first step, two Co. fioriniae strains (HC89 and HC91) from our collection that were previously isolated from apple were assayed for cercosporin production using the $\mathrm{KOH}$ assay (50). No cercosporin-like pigment was observed in the medium under the same conditions that stimulate cercosporin production in C. beticola. Since epigenetic modifiers have been used to induce production of SMs in fungal species $(51,52)$, we considered whether this strategy could be used to induce cercosporin production in Co. fioriniae. Medium augmented with the histone deacetylase inhibitor trichostatin A (TSA) (51) induced production of a red cercosporin-like compound. To characterize this red metabolite, mycelia from both Co. fioriniae strains were extracted with ethyl acetate. Reverse-phase HPLC analysis of extracts of both strains revealed a peak with a retention time and UV-vis spectrum consistent with cercosporin in both extracts (Fig. 7A, B). The presence of cercosporin was confirmed by UPLC-ESI-MS (Fig. 7C). Moreover, complementary re-sequencing of both isolates on the Illumina platform followed by automated genome assembly and gene prediction confirmed the presence of a CTB cluster in both genomes (Suppl. Table S8, Fig. S11). 


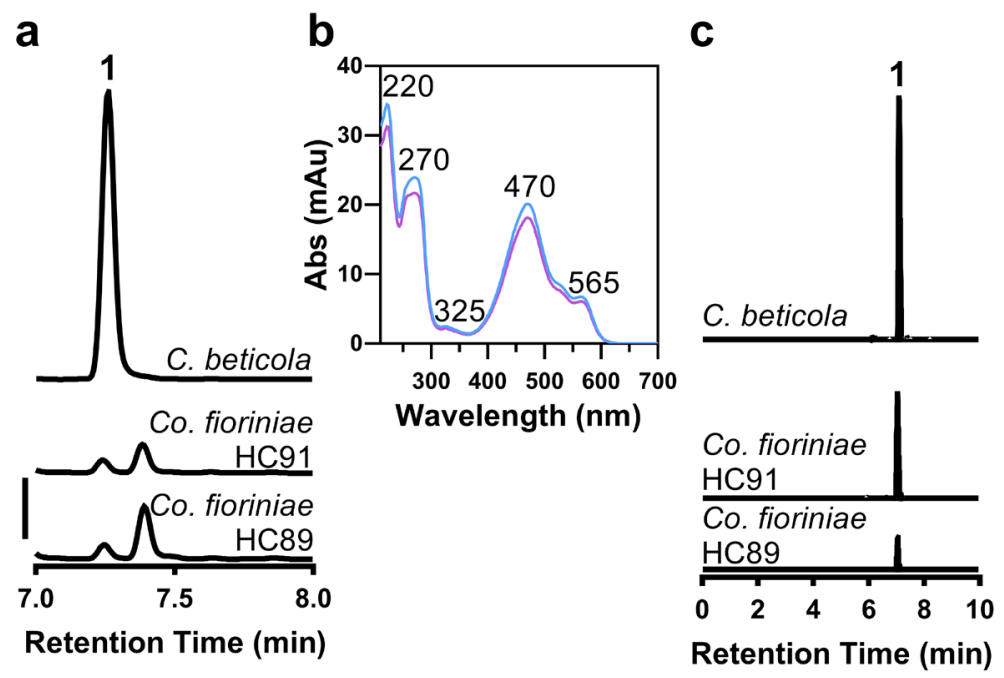

Figure 7. HPLC and UPLC-ESI-MS analysis of Colletotrichum fioriniae strains. a) HPLC chromatograms at $280 \mathrm{~nm}$ of wild-type C. beticola and Co. fioriniae HC89 and HC91. Scale bar indicates $100 \mathrm{mAu}$. b) UV-Vis spectra of cercosporin $(7.25 \mathrm{~min}$. retention timel extracted from Co. fioriniae HC89 (blue) and HC91 (purple). Wavelengths of relevant UV maxima are indicated. c) Extracted ion chromatograms ( $\mathrm{m} / \mathrm{z}=535.1604)$ obtained by UPLC-ESI-MS, demonstrating cercosporin production in C. beticola and Co. fioriniae strains HC89 and HC91.

To assess whether Co. fioriniae produces cercosporin during apple infection, apples were inoculated with the pathogen, and tissue samples were collected every other day from one to 14 days post inoculation. Regardless of time-point or extraction methodology, we were unable to detect cercosporin from infected apple. However, by infiltrating apples with defined amounts of cercosporin, we determined the isolation efficiency is approximately $5 \%$ (Suppl. Fig. S12, S13). Consequently, any cercosporin produced by Co. fioriniae during infection is likely rapidly bound or modified by apple tissue such that its recovery by extraction is poor and below our level of detection by HPLC or ESI-MS. To provide additional support for the involvement of cercosporin in Co. fioriniae infection, we infiltrated cercosporin into apple fruit and visually compared the phenotype of the infiltrated area to Co. fioriniae-infected apple. At five days post infiltration/inoculation, cercosporin-infiltrated apple exhibited similar symptomology as Co. fioriniae-infected apple (Suppl. Fig. S14). We also quantified expression of CTB1 using qRT-PCR since it is highly-expressed during cercosporin biosynthesis and is the first committed step in the pathway (21). In concordance with earlier results where no cercosporin was detected from cultures grown in vitro in the absence of TSA, we likewise did not detect CTB1 expression from this source (Suppl. Fig. S15). However, CTB1 was found to be induced $~ 11.9$-fold in in vitro cultures with TSA and 2.5-fold in apple samples harvested 14 days post inoculation (Suppl. Fig. S15). Since amplification products were designed to span introns, amplicons were sequenced and verified to be derived from cDNA. 
We also attempted to isolate cercosporin from Co. higginsianum-infected Arabidopsis leaves since previous studies indicated the distinct induction of this cluster from in planta appressoria (22 hours post inoculation; HPI) and the biotrophic phase of infection (40 HPI) but repressed during in vitro growth and the necrotrophic phase of infection (60 HPI) (44). Again, we were unable to detect cercosporin from infected Arabidopsis leaves regardless of the extraction methodology or time-point after inoculation. Given the relatively low amount of fungal biomass associated with the early time points when the CTB cluster is induced during Arabidopsis infection, we hypothesize that the amount of cercosporin produced in planta is below our current limits of detection.

\section{Discussion}

Several hypotheses exist for the maintenance of SM biosynthetic genes as clusters. In one, unlinked SM pathway genes are at a greater risk for dissociation during meiotic recombination (53) or chromosomal rearrangements (54). Additionally, clustering may facilitate strict coordination of gene expression, which may be particularly important during the biosynthesis of SMs that have potentially toxic or chemically unstable intermediates to ensure their efficient conversion to final end products (55). Horizontal transfer and maintenance of the CTB cluster specifically among plant pathogens suggests that it was critical for disease development in diverse pathosystems, including rice blast caused by M. oryzae and various anthracnose diseases caused by Colletotrichum spp. on many different crops. The CTB clusters in Co. higginsianum and Co. graminicola were reported as one of the few SM clusters between these species that are microsyntenic (44). Moreover, O'Connell et al. detected specific upregulation of the CTB cluster in Co. higginsianum during colonization of Arabidopsis (44). Indeed, nine of 14 Co. higginsianum CTB genes were among the top 100 most highly expressed genes in planta. Recent analysis of natural selection processes in Co. graminicola identified orthologs of CTB genes CTB1 and CFP among the 80 genes undergoing significant positive selection (56), further suggesting a role in pathogenicity. Interestingly, the CTB clusters of Colletotrichum spp. and M. oryzae contain additional genes; two short-chain dehydrogenases, an additional desaturase, a ferric-chelate reductase and an NmrA-like family protein, which has been reported (57) to act as negative transcriptional regulator.

The identification of cercosporin production in two isolates of Co. fioriniae may have significant implications for the apple packing, storage, and processing industries. Bitter rot, caused by Colletotrichum spp., is one of the top pre- and postharvest pathogens of apple (58). This disease is a major problem for the apple industry as it limits fresh fruit in the field and during storage, and has a quiescent stage allowing decay to occur on seemingly high-quality apples, only to come out of storage rotten $(58,59)$. Hence, contamination of processed apple products with cercosporin could be a significant health hazard. For example, other fungal-produced toxins (e.g. patulin, citrinin, penicillic acid) can contaminate processed apple products (60). Patulin, produced by Penicillium spp., is the most troubling as it is carcinogenic and consequently the United States and Europe have strict patulin limits in fruit juices and processed pome fruit products $(60,61)$. Future studies will focus on the role of cercosporin production during the Colletotrichum-apple fruit interactions in addition to assaying processed fruit products made 
from apples with bitter rot symptoms to determine levels of the toxin in fruit. Although only Co. fioriniae and Co. higginsianum strains were analyzed for the ability to produce cercosporin, the identification of highly similar CTB clusters in other Colletotrichum species (Suppl. Table S8, Fig. S10, S11) suggest that cercosporin production may be wide-spread in this genus. Future studies directed towards analysis of CTB cluster expression among various Co. fioriniae isolates and apple cultivars will be necessary to confirm whether cercosporin is necessary for virulence of this pathogen.

The microsynteny outside of the established CTB cluster prompted us to test whether the flanking genes in C. beticola are also required for cercosporin biosynthesis. Notably, we observed that these flanking genes, similar to the established CTB genes, were up-regulated under cercosporininducing conditions. Furthermore, targeted gene replacement of CTB9, CTB10, CDCTB11, and CTB12 completely abolished cercosporin biosynthesis, while replacement of CTB9 and CTB10 resulted in the accumulation of a new, red metabolite, defined here as pre-cercosporin. We thus conclude that the CTB cluster is significantly larger than previously described (19).

The isolation and characterization of a new intermediate in the cercosporin biosynthetic pathway, pre-cercosporin, strongly suggests that formation of the unique 7-membered methylenedioxy bridge in the final product is the result of a two-step process requiring three genes. First, one of two precursor aryl methoxyl groups of ent (+)-calphostin D (Fig. 6) is removed, followed by oxidative ring closure by CTB9, an apparent a-ketoglutarate-dependent dioxygenase, in collaboration with CTB10. The precise role of CTB10, a putative dehydratase, in ring closure is unclear, but it could serve to facilitate closure of the unfavorable 7-membered methylenedioxy ring. In contrast, a single cytochrome P450 is known to convert two aryl ortho-methoxyl groups into the relatively more common 5-membered methylenedioxy group in alkaloid biosynthesis (62). We attribute the single demethylation to an oxidative process possibly carried out by the flavin-dependent enzymes CTB5 or CTB7. CTB6 correlates to the SDR NAD(P)H-binding superfamily of oxidoreductases and could install the side chain hydroxyl groups stereospecifically. Owing to the extreme instability of most pathway intermediates and the role feedback inhibition in response to these metabolites could play, our experience dictates that analysis of pathway knockouts alone will not lead to the full determination of cercosporin biosynthesis. Biochemical evaluation of the individual enzymes, as has been done with CTB3 (21), with synthetic substrates and product standards will be necessary to accomplish this task.

A tentative cercosporin biosynthesis scheme was recently proposed (21) without knowledge of the expanded CTB cluster. However, in light of the identification of pre-cercosporin and the potential functions of the other newly discovered CTB genes, the previously proposed biosynthetic pathway (21) will have to be revised. While these investigations will be reported in due course, we suspect the newly discovered fasciclin/laccase pair (CTB11/12) may act early in the pathway to dimerize the product of CTB3 (21) to the first perylenequinone intermediate, which would have precedent in synthetic chemistry (63) and in simpler laccase-mediated arylaryl dimerizations $(36,64,65)$ (Fig. 6). CTB1 is an iterative, non-reducing polyketide synthase whose product is nor-toralactone (20). CTB3 is a bifunctional enzyme, O-methyltransferase 
and FAD-dependent monooxygenase, that carries out sequential O-methylation in the presence of S-adenosylmethionine (SAM) and oxidative decarboxylation to cercoquinone C (21). We hypothesize intermediate steps of $O$-dimethylation and side chain ketone reduction, in unspecified order, are mediated by CTB2 and potentially CTB6, respectively, as noted above.

Despite sustained research on cercosporin for several decades, there are significant knowledge gaps in cercosporin biosynthesis. Our data shed new light on cercosporin biology that will have significant impact on cercosporin research specifically and perylenequinone research in general. The finding that at least one species in the important plant pathogenic genus Colletotrichum can produce cercosporin has significant implications for disease management. Moreover, since Co. fioriniae may secrete cercosporin into apple food products that may be directly consumed by humans, the toxic effects of cercosporin on human health may need to be considered.

\section{Materials and Methods}

For further information, see SI Appendix Materials and Methods and figshare under DOI: 10.6084/ m9.figshare.4056522. Custom code is permanently archived at Zenodo under DOI: 10.5281/ zenodo.1156551.

Fungal genomic DNA was isolated from mycelia scraped from the surface of agar Petri plates. Library preparations and sequencing on the Illumina platform was performed by BGI Americas Corporation. For C. beticola, three genomic libraries with increasing insert size (500 bp, 5 Kbp, $10 \mathrm{Kbp}$ ) were sequenced. For C. berteroae, C. nicotianae, and for Co. fioriniae strains HC89 and HC91, single, short insert libraries (500 bp) were sequenced. For C. beticola specifically, optical maps were prepared using the Argus (OpGen) and BioNano Genomics platforms and subsequently used to scaffold contigs into large supercontigs. A combination of ab initio gene prediction, homologous protein alignment, and transcript alignment followed by extensive manual curation was used to prepare draft gene models for C. beticola. C. beticola trained Augustus parameters were used for automated protein-coding gene modelling in the case of C. berteroae, C. canescens and C. nicotianae. Genome assemblies and annotations, if applicable, are deposited at NCBI GenBank and listed under BioProject PRJA270309. Accession numbers for C. beticola, C. berteroae, C. nicotianae and Co. fioriniae strains HC89 and HC91 are: LKMD00000000, PNEN00000000, POSS00000000, and PNFH00000000 and PNFI00000000, respectively.

Mycelial plugs of wild-type and mutant C. beticola were placed on top of eight "thin" potato dextrose agar (PDA, Difco) plates (3.0 mL PDA per $50 \mathrm{~mm}$ Petri plate). Cultures were incubated at $22{ }^{\circ} \mathrm{C}$ for one week under continuous light. PDA and mycelia were ground under liquid nitrogen and lyophilized to dryness twice. The resulting powder was resuspended in water acidified with $\mathrm{HCl}(\mathrm{pH}<1)$, allowed to sit 10 minutes, and filtered. The filtrate was extracted thrice with ethyl acetate. These extracts were pooled washed with brine, and evaporated to dryness. The extracted metabolites were resuspended in $500 \mu \mathrm{L}$ methanol and analyzed by HPLC on an Agilent 1200 fitted with a Kinetex XB-C18 column (4.6 x 75 mm, $2.6 \mu$, Phenomenex). Injections 
of $1 \mathrm{\mu L}$ were run at $1.25 \mathrm{~mL} / \mathrm{min}$ with a linear gradient of $5 \% \mathrm{~A} / 95 \% \mathrm{~B}$ to $95 \% \mathrm{~A} / 5 \% \mathrm{~B}$ over 10.8 min, where solvent $A$ was acetonitrile $+0.1 \%$ formic acid and solvent $B$ was $0.1 \%$ formic acid. Chromatograms were monitored at 436, 280, and $210 \mathrm{~nm}$, and UV-vis spectra were recorded over a range of 210-800 $\mathrm{nm}$. High resolution mass data were obtained from a Waters Acquity/ Xevo-G2 UPLC-ESI-MS in positive ion mode.

Acknowledgements We thank W. Underwood and T. L. Friesen (USDA - ARS) for review of the manuscript, A. G. Newman for helpful discussions and a reference sample of cercosporin and N. Metz (USDA - ARS) for excellent technical assistance. Prof. M. Muller and L. Fürtges (Univ. Freiburg) are thanked for discussion of fungal laccases and unpublished results. This project was supported by an EMBO Long-Term Fellowship (ALTF 359-2013) and a postdoctoral fellowship of the Research Foundation Flanders (FWO 12B8116N) to R. de J., USDA-ARS CRIS project 3060-22000-049 to M. D. B. and by The National Institutes of Health grant RO1 ES001670 to C. A. T and T32 GM080189 to C. R. H.-R. Mention of trade names or commercial products in this publication is solely for the purpose of providing specific information and does not imply recommendation or endorsement by the U.S. Department of Agriculture.

\section{Supporting material for this chapter can be accessed under https://doi.org/10.1073/ pnas. 1712798115}

\section{References}

1. Groenewald M, Groenewald JZ, Braun U, \& Crous PW (2006) Host range of Cercospora apii and C. beticola and description of C. apiicola, a novel species from celery. Mycologia 98(2):275-285.

2. Fuckel K (1863) Fungi rhenani exsiccati, fasc. I-iv. Hedwigia 2:132-136.

3. Secor GA, Rivera VV, Khan MFR, \& Gudmestad NC (2010) Monitoring fungicide sensitivity of Cercospora beticola of sugar beet for disease management decisions. Plant Disease 94(11):1272-1282.

4. Wrather A, et al. (2010) Effect of diseases on soybean yield in the top eight producing countries in 2006. Plant Health Progress 10:1094

5. Ward JMJ, Stromberg EL, Nowell DC, \& Nutter FW (1999) Gray leaf spot: A disease of global importance in maize production. Plant Dis 83(10):884-895.

6. Mani KK, Hollier CA, \& Groth DE (2017) Effect of cultivar susceptibility and planting date on narrow brown leaf spot progression in rice. Crop Prot 102(Supplement C):88-93.

7. Uppala S \& Zhou XG (2018) Field efficacy of fungicides for management of sheath blight and narrow brown leaf spot of rice. Crop Prot 104(Supplement C):72-77.

8. Olatinwo RO, Prabha TV, Paz JO, \& Hoogenboom G (2012) Predicting favorable conditions for early leaf spot of peanut using output from the weather research and forecasting (WRF) model. Int J Biometeorol 56(2):259-268.

9. Stergiopoulos I, Collemare J, Mehrabi R, \& De Wit PJGM (2013) Phytotoxic secondary metabolites and peptides produced by plant pathogenic Dothideomycete fungi. FEMS Microbiol Rev 37(1):67-93.

10. Goodwin SB \& Dunkle LD (2010) Cercosporin production in Cercospora and related anamorphs of Mycosphaerella. Cercospora leaf spot of sugar beet and related species, eds Lartey RT, Weiland JJ, Panella L, Crous PW, \& Windels CE (The American Phytopathological Society), pp 97-108.

11. Daub ME \& Ehrenshaft M (2000) The photoactivated Cercospora toxin cercosporin: Contributions to plant disease and fundamental biology. Annu Rev Phytopathol 38(1):461-490.

12. Daub ME \& Hangarter RP (1983) Light-induced production of singlet oxygen and superoxide by the fungal toxin, cercosporin. Plant Physiol 73(3):855-857.

13. Dobrowolski DC \& Foote CS (1983) Cercosporin, a singlet oxygen generator. Angew Chem, Int Ed Engl 22(9):720-721

14. Daub ME (1982) Cercosporin, a photosensitizing toxin from Cercospora species. Phytopathol 72(4):370-374.

15. Gunasinghe N, You MP, Cawthray GR, \& Barbetti MJ (2016) Cercosporin from Pseudocercosporella capsellae and its critical role in white leaf spot development. Plant Dis 100(8):1521-1531.

16. Crous PW, et al. (2013) Phylogenetic lineages in Pseudocercospora. Stud Mycol 75(1):37-114.

17. Daub ME (1981) Destruction of tobacco cell-membranes by the photosensitizing toxin, cercosporin. Phytopathol $71(8): 869-869$. 
18. Daub ME (1987) Resistance of fungi to the photosensitizing toxin, cercosporin. Phytopathol 77(11):1515-1520.

19. Chen HQ, Lee MH, Daub ME, \& Chung KR (2007) Molecular analysis of the cercosporin biosynthetic gene cluster in Cercospora nicotianae. Mol Microbiol 64(3):755-770.

20. Newman AG, Vagstad AL, Belecki K, Scheerer JR, \& Townsend CA (2012) Analysis of the cercosporin polyketide synthase CTB1 reveals a new fungal thioesterase function. Chem Commun 48(96):11772-11774.

21. Newman AG \& Townsend CA (2016) Molecular characterization of the cercosporin biosynthetic pathway in the fungal plant pathogen Cercospora nicotianae. J Am Chem Soc 138(12):4219-4228.

22. Choquer M, Lee MH, Bau HJ, \& Chung KR (2007) Deletion of a MFS transporter-like gene in Cercospora nicotianae reduces cercosporin toxin accumulation and fungal virulence. FEBS Lett 581(3):489-494.

23. Callahan TM, Rose MS, Meade MJ, Ehrenshaft M, \& Upchurch RG (1999) CFP, the putative cercosporin transporter of Cercospora kikuchii, is required for wild type cercosporin production, resistance, and virulence on soybean. Mol Plant-Microbe Interact 12(10):901-910.

24. Chand R, et al. (2015) Draft genome sequence of Cercospora canescens: A leaf spot causing pathogen. Curr Sci 109(11):2103-2110.

25. Blin K, et al. (2013) Antismash 2.0-a versatile platform for genome mining of secondary metabolite producers. Nucleic Acids Res 41(W1):W204-W212.

26. Ohm RA, et al. (2012) Diverse lifestyles and strategies of plant pathogenesis encoded in the genomes of eighteen Dothideomycetes fungi. PLoS Pathogens 8(12):e1003037.

27. Collemare J, et al. (2014) Secondary metabolism and biotrophic lifestyle in the tomato pathogen Cladosporium fulvum. PLOS ONE 9(1):e85877.

28. Medema MH, Cimermancic P, Sali A, Takano E, \& Fischbach MA (2014) A systematic computational analysis of biosynthetic gene cluster evolution: Lessons for engineering biosynthesis. PLoS Computational Biology 10(12):e1004016.

29. Chooi Y-H, Muria-Gonzalez MJ, \& Solomon PS (2014) A genome-wide survey of the secondary metabolite biosynthesis genes in the wheat pathogen Parastagonospora nodorum. Mycology 5(3):192-206.

30. Koczyk G, Dawidziuk A, \& Popiel D (2015) The distant siblings - a phylogenomic roadmap illuminates the origins of extant diversity in fungal aromatic polyketide biosynthesis. Genome Biol Evol 7(11):3132-3154.

31. Stolzer M, et al. (2012) Inferring duplications, losses, transfers and incomplete lineage sorting with nonbinary species trees. Bioinformatics 28(18):i409-i415.

32. Tudzynski B, et al. (2003) Characterization of the final two genes of the gibberellin biosynthesis gene cluster of Gibberella fujikuroi: des and p450-3 encode GA4 desaturase and the 13-hydroxylase, respectively. J Biol Chem 278(31):28635-28643.

33. Kim J-E, et al. (2005) Putative polyketide synthase and laccase genes for biosynthesis of aurofusarin in Gibberella zeae. Appl Environ Microbiol 71(4):1701-1708.

34. Williams JS, Thomas M, \& Clarke DJ (2005) The gene stlA encodes a phenylalanine ammonia-lyase that is involved in the production of a stilbene antibiotic in Photorhabdus luminescens TT01. Microbiol 151(8):2543-2550.

35. Choquer M, Lee M-H, Bau H-J, \& Chung K-R (2007) Deletion of a mfs transporter-like gene in Cercospora nicotianae reduces cercosporin toxin accumulation and fungal virulence. FEBS Letters 581(3):489-494.

36. Frandsen RJN, et al. (2011) Two novel classes of enzymes are required for the biosynthesis of aurofusarin in Fusarium graminearum. J Biol Chem 286(12):10419-10428.

37. Gao Q, et al. (2011) Genome sequencing and comparative transcriptomics of the model entomopathogenic fungi Metarhizium anisopliae and M. acridum. PLOS Genet 7(1):e1001264.

38. Shimodaira $H$ (2002) An approximately unbiased test of phylogenetic tree selection. Syst Biol 51(3):492-508

39. Shimodaira H \& Hasegawa M (2001) Consel: For assessing the confidence of phylogenetic tree selection. Bioinformatics 17(12):1246-1247.

40. Wisecaver JH \& Rokas A (2015) Fungal metabolic gene clusters-caravans traveling across genomes and environments. Frontiers in Microbiology 6:161.

41. Velho AC, Stadnik MJ, Casanova L, Mondino P, \& Alaniz S (2013) First report of Colletotrichum nymphaeae causing apple bitter rot in southern Brazil. Plant Dis 98(4):567-567.

42. Víchová J, Vejražka K, Cholastová T, Pokorný R, \& Hrudová E (2010) Colletotrichum simmondsii causing anthracnose on safflower in the Czech Republic. Plant Dis 95(1):79-79.

43. Cannon PF, Damm U, Johnston PR, \& Weir BS (2012) Colletotrichum - current status and future directions. Stud Mycol 73:181-213.

44. O'Connell RJ, et al. (2012) Lifestyle transitions in plant pathogenic Colletotrichum fungi deciphered by genome and transcriptome analyses. Nat Genet 44(9):1060-1065.

45. Sukno SA, García VM, Shaw BD, \& Thon MR (2008) Root infection and systemic colonization of maize by Colletotrichum graminicola. Appl Environ Microbiol 74(3):823-832.

46. Weir BS, Johnston PR, \& Damm U (2012) The Colletotrichum gloeosporioides species complex. Stud Mycol 73:115-180.

47. Damm U, et al. (2013) The Colletotrichum orbiculare species complex: Important pathogens of field crops and weeds. Fungal Divers 61(1):29-59.

48. Damm U, Cannon PF, Woudenberg JHC, \& Crous PW (2012) The Colletotrichum acutatum species complex. Stud Mycol 73(Supplement C):37-113.

49. Kasson MT, Pollok JR, Benhase EB, \& Jelesko JG (2014) First report of seedling blight of eastern poison ivy (Toxicodendron radicans) by Colletotrichum fioriniae in Virginia. Plant Dis 98(7):995-995. 
50. Choquer M, et al. (2005) The CTB1 gene encoding a fungal polyketide synthase is required for cercosporin biosynthesis and fungal virulence of Cercospora nicotianae. Mol Plant-Microbe Interact 18(5):468-476.

51. Shwab EK, et al. (2007) Histone deacetylase activity regulates chemical diversity in Aspergillus. Eukaryot Cell 6(9):1656-1664.

52. Williams RB, Henrikson JC, Hoover AR, Lee AE, \& Cichewicz RH (2008) Epigenetic remodeling of the fungal secondary metabolome. Org Biomol Chem 6(11):1895-1897.

53. Galazka JM \& Freitag M (2014) Variability of chromosome structure in pathogenic fungi - of 'ends and odds'. Curr Opin Microbiol 20(0):19-26.

54. de Jonge R, et al. (2013) Extensive chromosomal reshuffling drives evolution of virulence in an asexual pathogen. Genome Res 23(8):1271-1282.

55. McGary KL, Slot JC, \& Rokas A (2013) Physical linkage of metabolic genes in fungi is an adaptation against the accumulation of toxic intermediate compounds. Proc Natl Acad Sci U S A 110(28):11481-11486.

56. Rech GE, Sanz-Martín JM, Anisimova M, Sukno SA, \& Thon MR (2014) Natural selection on coding and noncoding DNA sequences is associated with virulence genes in a plant pathogenic fungus. Genome Biol Evol 6(9):2368-2379.

57. Stammers DK, et al. (2001) The structure of the negative transcriptional regulator NmrA reveals a structural superfamily which includes the short-chain dehydrogenase/reductases. The EMBO Journal 20 (23):6619-6626.

58. Munir M, Amsden B, Dixon E, Vaillancourt L, \& Gauthier NAW (2016) Characterization of Colletotrichum species causing bitter rot of apple in Kentucky orchards. Plant Dis 100(11):2194-2203.

59. Kou LP, Gaskins V, Luo YG, \& Jurick WM (2013) First report of Colletotrichum fioriniae causing postharvest decay on 'nittany' apple fruit in the United States. Plant Dis 98(7):993-993.

60. Wright SAI (2015) Patulin in food. Curr Opin Food Sci 5:105-109.

61. Puel O, Galtier P, \& Oswald I (2010) Biosynthesis and toxicological effects of patulin. Toxins 2(4):613.

62. Díaz Chávez ML, Rolf M, Gesell A, \& Kutchan TM (2011) Characterization of two methylenedioxy bridgeforming cytochrome P450-dependent enzymes of alkaloid formation in the mexican prickly poppy Argemone mexicana. Arch Biochem Biophys 507(1):186-193.

63. Hauser FM, Sengupta D, \& Corlett SA (1994) Optically active total synthesis of calphostin D. J Org Chem 59(8):1967-1969.

64. Bode SE, Drochner D, \& Müller M (2007) Synthesis, biosynthesis, and absolute configuration of vioxanthin. Angewandte Chemie 119(31):6020-6024.

65. Kawaguchi M, et al. (2018) Discovery of a fungal multicopper oxidase that catalyzes the regioselective coupling of a tricyclic naphthopyranone to produce atropisomers. Angewandte Chemie 0(0):in press. 



\section{Gene cluster conservation identifies melanin and perylenequinone biosynthesis pathways in multiple plant pathogenic fungi}

Malaika K. Ebert*, Rebecca E. Spanner*, Ronnie de Jonge*,\#,

David J. Smith, Jason Holthusen, Gary A. Secor, Bart P.H.J. Thomma, and Melvin D. Bolton\#

* These authors contributed equally to this work.

\#These authors contributed equally to this work. 


\section{Abstract}

Perylenequinones are a family of structurally related polyketide fungal toxins with nearly universal toxicity. These photosensitizing compounds absorb light energy which enable them to generate reactive oxygen species that damage host cells. This potent mechanism serves as an effective weapon for plant pathogens in disease establishment. The sugar beet pathogen Cercospora beticola secretes the perylenequinone cercosporin during infection. We have shown recently that the cercosporin toxin biosynthesis (CTB) gene cluster is present in several other phytopathogenic fungi, prompting the search for biosynthetic gene clusters (BGCs) of structurally similar perylenequinones in other fungi. Here, we report the identification of the elsinochrome and phleichrome BGCs of Elsinoe fawcettii and Cladosporium phlei, respectively, based on gene cluster conservation with the CTB and hypocrellin BGCs. Furthermore, we show that previously reported BGCs for elsinochrome and phleichrome are involved in melanin production. Phylogenetic analysis of the corresponding melanin polyketide synthases (PKSs) and alignment of melanin BGCs revealed high conservation between the established and newly identified C. beticola, E. fawcettii, and C. phlei melanin BGCs. Mutagenesis of the identified perylenequinone and melanin PKSs in C. beticola and E. fawcettii coupled with mass spectrometric metabolite analyses confirmed their roles in toxin and melanin production. 


\section{Introduction}

Fungi produce a plethora of secondary metabolites (SMs) that serve to enhance competitiveness in nature. Functional diversity of these compounds is high, including reported roles in virulence, biotic and abiotic stress protection, and as metal transport agents (1-5). For example, in some occasions SMs are involved in symbiotic relationships where microbial symbionts provide an antibiotic armory against secondary infection to the symbiotically colonized plant in return for nutrients and protection (5). A major class of fungal SMs are the polyketides (6). For the biosynthesis of fungal aromatic polyketides, non-reducing polyketide synthases (NR-PKSS) play a central role as mediators of the first biosynthetic step (6-9). Such PKS genes contain multiple domains that work conjointly, of which the $\beta$-ketoacyl synthase (KS), acyltransferase (AT), and acyl-carrier protein (ACP) domain are indispensable $(6,7,9,10)$. By using the domains iteratively, a PKS generates a metabolite backbone which can be modified by other enzymes to yield the final metabolite $(6,9,11)$. The genes encoding these decorating enzymes are often found in direct proximity to the PKS gene to form a biosynthetic gene cluster (BGC) pathway $(6,12)$. In addition, BGCs contain regulatory elements and transporters involved in shuttling the final secondary metabolite from the cell, and in the case of toxic metabolites, genes encoding autoresistance proteins $(4,13)$.

A well-studied BGC is the cercosporin toxin biosynthesis (CTB) pathway. The CTB gene cluster was originally identified in Cercospora nicotianae, causal agent of leaf spot disease on tobacco, but is present in almost all Cercospora species (13-15). The ubiquitous presence of the CTB gene cluster in the genus is likely explained by its role as a virulence facilitator (15-17). Recently, de Jonge et al. (2018) used comparative genomics to show that the CTB gene cluster can also be found in several plant pathogenic fungal species outside the Cercospora genus, likely as a result of horizontal transfer of the entire CTB gene cluster $(9,11,13)$. The majority of assessed species from the genus Colletotrichum, a large genus of crop and/or ornamental plant pathogens (18), were shown to harbor full- to partial-length CTB gene clusters, of which the post-harvest apple fruit pathogen Co. fioriniae was shown to produce cercosporin (13). The core gene of the Cercospora CTB gene cluster is the NR-PKS gene CTB1 (19), which is flanked at both sides by nine genes that putatively encode decorating enzymes (CTB2, CTB3, CTB5, CTB6, CTB7, CTB9, CTB10, CTB11 and CTB12) (13). Besides those ten genes essential for toxin formation, the cluster also encodes a zinc finger transcription factor (CTB8) for regulation of cluster gene expression, and two major facilitator superfamily (MFS) transporters; CTB4 that is necessary for toxin secretion and the cercosporin facilitator protein (CFP) involved in toxin auto-resistance (13, 20, 21). Upon activation, all CTB pathway enzymes work in a well-orchestrated manner to synthesize the metabolite from backbone formation to secretion of the toxin into the environment whilst providing the fungus with protection against cercosporin.

Cercosporin is a member of the perylenequinone family that, upon photo-activation, displays almost universal toxicity to a wide spectrum of organisms $(16,22-25)$. Exposure to visible and near-UV light energetically activates perylenequinones to an excited triplet state that reacts with oxygen to form reactive oxygen species $(25,26)$. This photodynamic activity can be attributed to the 3,10-dihydroxy-4,9-perylenequinone chromophore backbone that is shared among 
perylenequinones (27). Structural differences between perylenequinone family members are mostly due to divergent side chains attached to the mutual backbone structure (28) (Fig. 1). For example, the methylenedioxy bridge is a unique feature of cercosporin and is absent in other perylenequinones such as hypocrellin, elsinochrome and phleichrome (Fig. 1) (13, 29).<smiles></smiles>

elsinochrome A<smiles>COc1cc(OC)c2c3c1[C@@H](C(C)O)[C@@H](C(C)O)c3c1c(OC)c(=O)c3c(O)cc(OC)c4c(O)cc(OC)c2c1=c43</smiles>

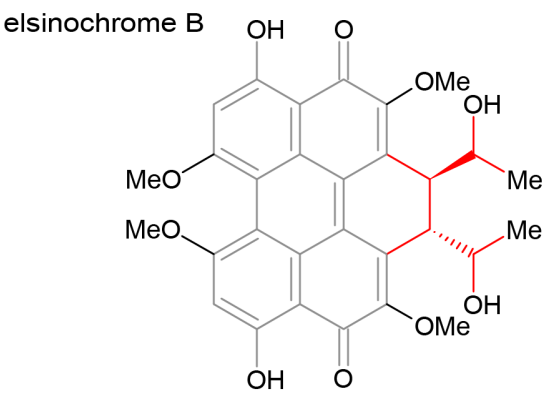

elsinochrome C

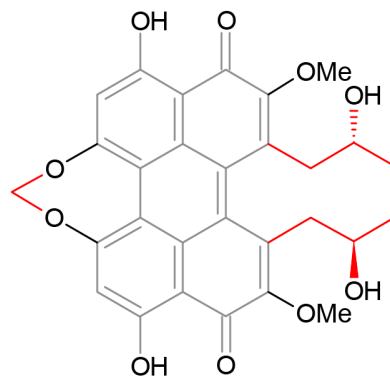

cercosporin

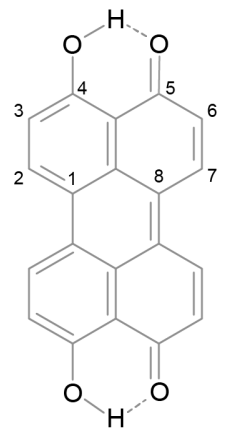

perylenequinone core<smiles></smiles>

phleichrome<smiles></smiles>

hypocrellin A

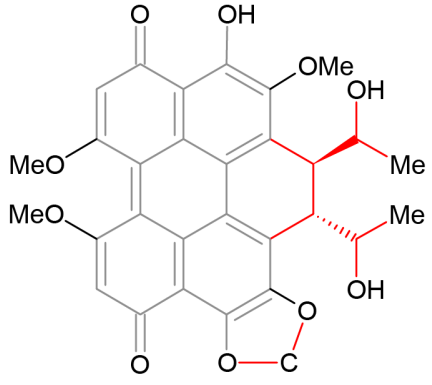

elsinochrome D

Figure 1. Structures of related perylenequinones. Cercosporin synthesized by Cercospora spp., phleichrome by C. phlei and elsinochromes A, B, C, and D produced by E. fawcettii are structurally related as they share a common perylenequinones backbone (center; indicated in the molecule structure in gray). Structural differences between the molecules are mostly due to various side chains (indicated in red). Differences between perylenequinones are observed at positions 2, 2'and 7, 7'. 
Previous studies have implicated PKS genes in the production of perylenequinones in other plant pathogenic fungi. For example, transcriptome analysis and a CRISPR-Cas9 gene editing approach in the bamboo pathogen Shiraia bambusicola gave compelling evidence that SbaPKS encodes the PKS orchestrating hypocrellin biosynthesis $(30,31)$. Similarly, targeted disruption of EfPKS1 in the citrus scab pathogen Elsinoë fawcettii appeared to abrogate elsinochrome production (32). Likewise, Cppks1 was suggested to be responsible for PKS activity for phleichrome production in the purple eyespot pathogen Cladosporium phlei (33). However, we have previously used KS domain phylogeny to associate PKS genes with the final perylenequinone product (13). During the course of these analyses, we identified PKS genes for E. fawcettii and C. phlei that were not previously attributed to these perylenequinones, which prompted us to re-evaluate the findings of Liao et al. (2008) (32) and So et al. (2015) (33). Interestingly, Liao et al. (2008) (32) also carried out phylogenetic analysis of EfPKS1 with other PKSs which indicated that EfPKS1 clustered closely to a diverse set of fungal nonreducing PKSs involved in biosynthesis of the toxins cercosporin, aflatoxin and sirodesmin, but also with PKSs involved in pigment production such as dihydroxynaphthalene (DHN)-melanin. Melanin is an integral component of the cell wall that has proposed functions in protection from environmental factors, appressorial penetration of host plants and pathogenesis (34-36). In Mycosphaerella fijiensis, research suggested that secreted fungal DHN-melanin acts as a virulence factor through the photogeneration of singlet molecular oxygen in a similar manner to the perylenequinones (37).

DHN-melanin biosynthesis has been characterized extensively in many fungi, including Magnaporthe oryzae, Colletotrichum lagenarium, Alternaria alternata, Botrytis cinerea, Verticillium dahliae and Aspergillus spp. In the rice blast fungus M. oryzae for instance, DHN-

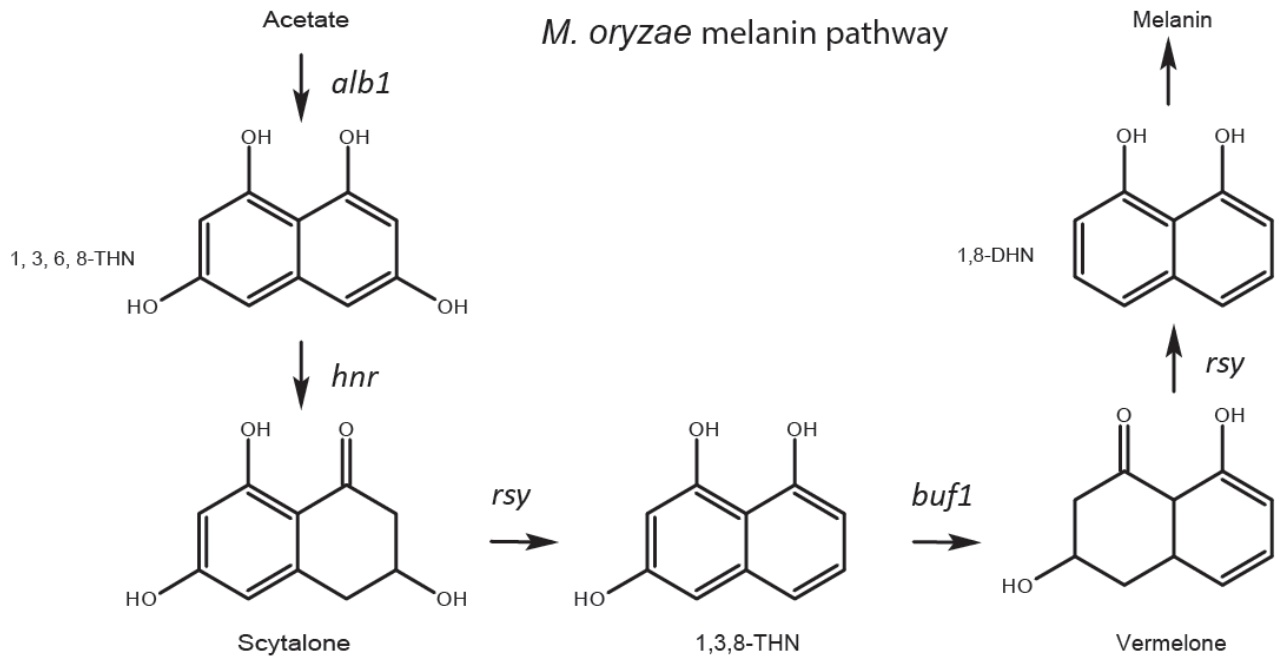

Figure 2. Schematic DHN-melanin biosynthesis pathway of M. oryzae. In the first biosynthetic step, the PKS ALB1 forms 1,3,6,8-tetrahydroxynaphthalene (1,3,6,8-THN or T4HN) by ketide cyclization. Reduction by the tetrahydroxynaphthalene reductase $4 \mathrm{HNR}$ results in the formation of scytalone which will be dehydrated by RYS1, a scytalone dehydratase, to yield trihydroxynaphthalene (T3HN). The T3HN reductase BUF1 subsequently reduces T3HN to vermelone followed by a dehydration step mediated by RYS1 to form dihydroxynaphthalene $(2 \mathrm{HN})$, the immediate precursor of melanin. 
melanin production is known to be mediated by a four-gene cluster which is regulated in hyphae by the transcription factor Pig1 (Fig. 2) (38-41). However, fungal DHN-melanin pathways may vary in the biosynthesis of the first common intermediate 1,3,6,8-tetrahydroxynaphthalene $(1,3,6,8$ THN or T4HN). For example, the PKS ALB1 (for "albino 1") is responsible for the first biosynthetic step in Aspergillus fumigatus, resulting in the biosynthesis of the heptaketide naphthopyrone YWA1, which is subsequently hydrolyzed by Ayg1 to produce T4HN $(42,43)$. Two alternative routes can be found in the necrotrophic gray mold fungus B. cinerea. In this case, the PKSs Bcpks12 and Bcpks13 synthesize different precursors for the joint DHN-melanin pathway (Schumacher, 2016). While Bcpks12 produces the pentaketide T4HN directly, Bcpks13 synthesizes the hexaketide 2-acetyl-1,3,6,8-tetrahydroxynaphthalene (AT4HN) that is subsequently converted to yield T4HN (44). In either case, the resulting T4HN will serve as substrate for a hydroxynaphthalene (HN) reductase leading to scytalone formation. In the next step, scytalone will be dehydrated by a scytalone dehydratase resulting in the formation of 1,3,8-trihydroxynapthalene $(1,3,8-\mathrm{THN}$ or $\mathrm{T} 3 \mathrm{HN}$ ). Subsequent reduction by a $\mathrm{HN}$ reductase yields vermelone which is subsequently dehydrated to form 1,8-DHN; an immediate precursor of melanin $(38-40,45)$.

In this manuscript, we show that the gene clusters housing CPpKs1 and EfPKS1 have high similarity to established gene clusters involved in DHN-melanin biosynthesis and have only limited similarity to the perylenequinone biosynthesis clusters to which they were previously attributed. Due to its detailed characterization, the established M. oryzae melanin cluster was used as reference in our alignments of putative DHN-melanin gene clusters of C. beticola, E. fawcettii, C. phlei, and S. bambusicola to illustrate the high level of homology among the DHN-melanin BGCS. Consequently, we also sought to establish the BGCs involved in production of elsinochrome in E. fawcettii and phleichrome in C. phlei, and included targeted gene replacement of both perylenequinone and melanin PKS genes in E. fawcettii and C. beticola to provide proof for their involvement in toxin and DHN-melanin production.

\section{Results}

\section{E. fawcettii and C. phlei genomics}

Nuclear and mitochondrial DNA of E. fawcettii strain CBS 139.25 and C. phlei strain CBS 358.69 were sequenced to approximately 138 -fold and 110 -fold coverage, respectively, on the Illumina platform (paired-end, 100-bp reads). Raw reads were processed and assembled by SPAdes (version 3.9.0) yielding draft genome assemblies of $25.3 \mathrm{Mb}$ on 398 scaffolds for E. fawcettii and $31.9 \mathrm{Mb}$ on 794 scaffolds for C. phlei. The respective scaffold N50 values and L50 numbers for these assemblies are 13 and $676 \mathrm{~Kb}$, and 44 and $238 \mathrm{~Kb}$. Following genome assembly, we used Augustus (version 3.2.1) with default settings (46) and the previously devised C. beticola training parameters (13) to predict 9,519 (mean length 1,675 bp and 2.5 exons/gene) and 11,316 (mean length 1,624 bp and $\sim 2.3$ exons/gene) protein-coding genes for E. fawcettii and C. phlei respectively. Finally, protein function as well as putative localization was predicted by Interpro (47) scanning and yielded annotations for 9,253 out of 9,519 E. fawcettii proteins and 10,870 out of 11,316 C. phlei proteins. Considering only hits to Pfam, SMART, CDD or SUPERFAMILY databases, $7,450(78 \%)$ and $8,479(75 \%)$ genes were annotated for E. fawcettii and C. phlei, respectively. 


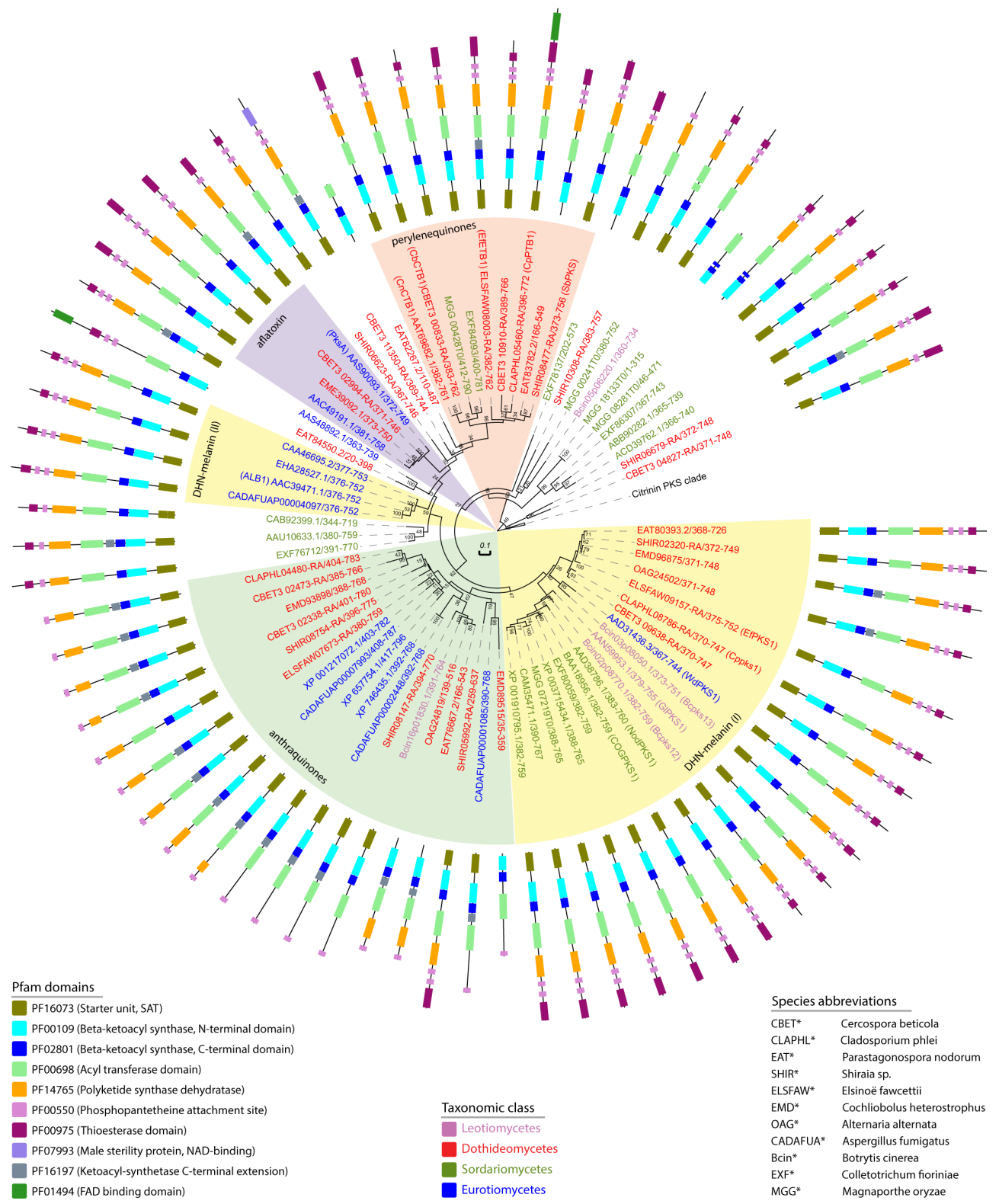

Figure 3. Phylogeny of PKS genes of related Ascomycetes revealing distinct DHN-melanin and perylenequinone subclades. Circular maximum likelihood phylogenetic tree illustrating the phylogenetic relationship of all predicted non-reducing polyketide synthase (PKS) from the selected species set (Suppl. Table 1) plus those derived from the set of PKSs used by Collemare et al. (2014) (48). The tree was constructed by maximum-likelihood analysis of aligned full-length $\beta$-ketoacyl synthase domains. The outside ring indicates domain architecture of each PKS determined by Pfam domain annotation. Protein accessions are colored depending on the taxonomic class of the producing species. The corresponding species identity for each protein can be found in the bottom left corner. Established biosynthetic end products for a subset of the listed PKSs is indicated by the background color, highlighting two DHN-melanin sub-groups, naphthoquinones, anthraquinones, perylenequinones, aflatoxin-like compounds and resorcylic acid lactones. 


\section{PKS genealogy and prediction of function}

To study the level of conservation of PKSs and associated pathways involved in the biosynthesis of different perylenequinones, we mined the genomes of both perylenequinone producers and non-producers for non-reducing PKSs (Suppl. Table 1). Subsequently, the phylogenetic relationships between these PKSS and those of previously characterized PKSs from selected species as found in Collemare et al. (2014) (48) (Suppl. Table 1) were determined by aligning the highly conserved $\beta$-ketoacyl synthase (KS) domains of each PKS (Fig. 3). This genealogy revealed distinctive clade formation where PKSS with confirmed involvement in biosynthesis of structurally similar metabolites were observed to cluster. The clades were categorized as perylenequinone, aflatoxin, anthraquinone, or DHN-melanin biosynthesis depending on the function of confirmed PKSs they harbored (Fig. 3). Interestingly, the PKSs EfPKS1 (32)/ [ELSFAW09157-RA (this study)] from E. fawcettii and Cppks1 (33)/ [CLAPH08786-RA (this study)] from C. phlei that were previously implicated in perylenequinone biosynthesis did not cluster phylogenetically with the established perylenequinone cercosporin PKSs CbCTB1 and CnCTB1 of C. beticola and C. nicotianae, respectively. Instead, EfPKS1 and Cppks1 formed a clade with confirmed melanin PKSs, including Bcpks12 and Bcpks13 of the gray mold fungus B. cinerea (44), Wdpks1 of the zoopathogenic black yeast Wangiella (Exophiala) dermatitidis (49), GIPKS1 of the filamentous fungus Glarea lozoyensis (50), NodPKS1 of an endophytic Nodulisporium strain (51), and COGPKS1 of the cucumber anthracnose causal agent Co. lagenarium (52) and the predicted C. beticola melanin biosynthesis PKS CbPKS1 (CBET3_09638) and the S. bambusicola melanin PKS SHIR08477. The finding that E. fawcettii ELSFAW09157-RA, C. phlei CLAPHL08786-RA, S. bambusicola SHIR08477, and CbPKS1 reside in a cluster with extensive collinearity to established DHN-melanin clusters (Fig. 3) suggests a role in melanin production and hints that EfPKS1 and Cppks1 were previously misannotated as perylenequinone biosynthesis genes $(32,33)$.

The cercosporin PKSs in C. beticola (CbCTB1), C. nicotianae (CnCTB1), and Co. fioriniae (EXF84093) form a perylenequinone clade with the previously confirmed hypocrellin PKS (SbaPKS) (30, 31), ELSFAW08003 from E. fawcettii, CLAPHL05460 from C. phlei as well as with the putative perylenequinone PKSs in P. nodorum (EAT83782.2), M. oryzae (MGG_00428), and the C. beticola CbCTB1 paralog CBET3_10910 (Fig. 3). As phylogenetic conservation can be an indication of related metabolite production (13), this clustering suggests that PKSs of this clade are involved in biosynthesis of the perylenequinones. Therefore, we suggest renaming ELSFAW08003 to EFETB1 for elsinochrome toxin biosynthesis gene 1, and C. phlei CLAPHL05460 to CPPTB1 for phleichrome toxin biosynthesis gene 1 .

\section{Perylenequinone and DHN-melanin biosynthesis gene cluster alignments}

While PKS genes are indispensable for polyketide formation, it is the full complement of genes in a $\mathrm{BGC}$ that is responsible for the biosynthesis of the end product. Therefore, synteny of the predicted BGCs of orthologous PKS genes was assessed. Using the established C. beticola CTB gene cluster and S. bambusicola hypocrellin gene cluster as references, putative perylenequinone orthologous gene clusters in E. fawcettii, C. phlei, P. nodorum, M. oryzae, and Co. fioriniae were aligned (Fig. 4A). Although there is evidence for gene loss and gain between the perylenequinone BGC alignments, multiple core genes are shared between cercosporin, hypocrellin, and the predicted BGCs for 


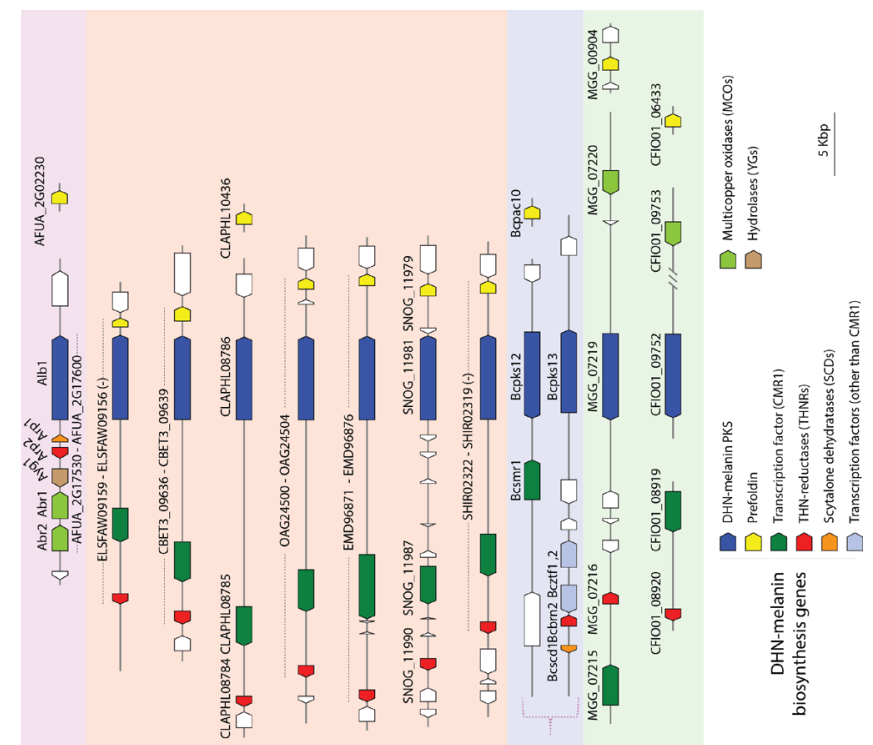

$\bar{\infty}$

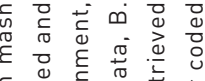

ᄃ

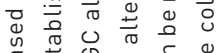

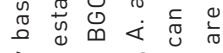

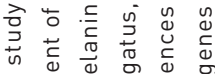

$\backsim \varepsilon \varepsilon \bar{\varepsilon} \vec{\sigma} 0$

三

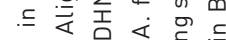

वें

ज

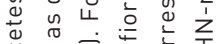

仓仓

¿

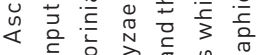

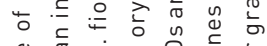

ब

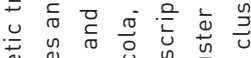

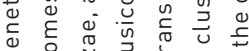

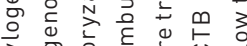
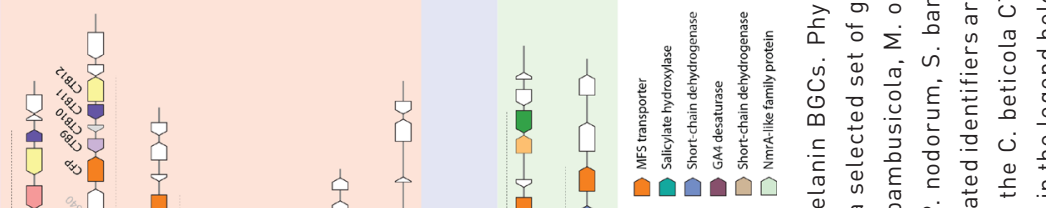

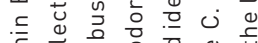

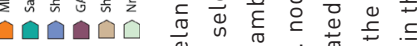
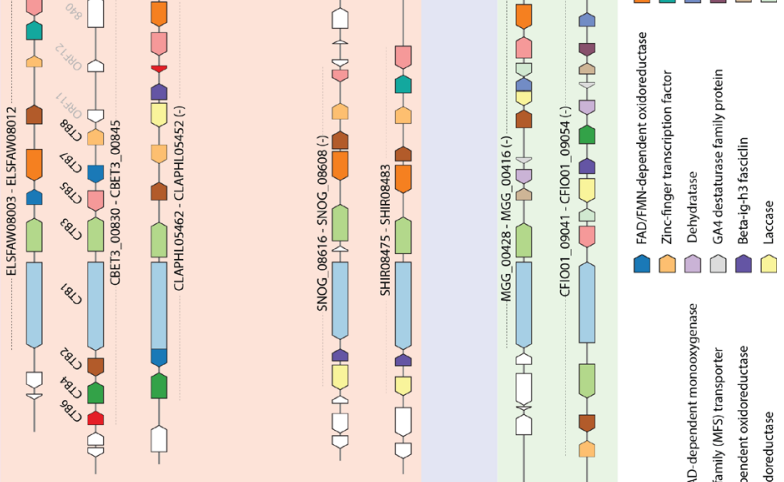

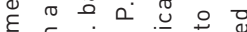

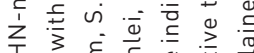

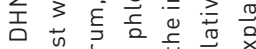

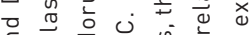

厂

¿

.

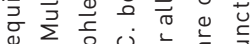

¿ 50.000

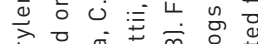

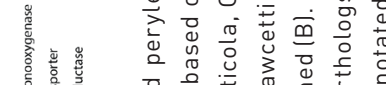

ग)

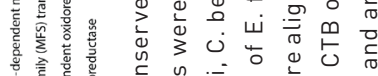

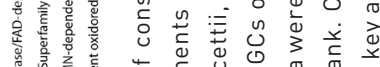

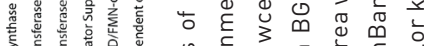

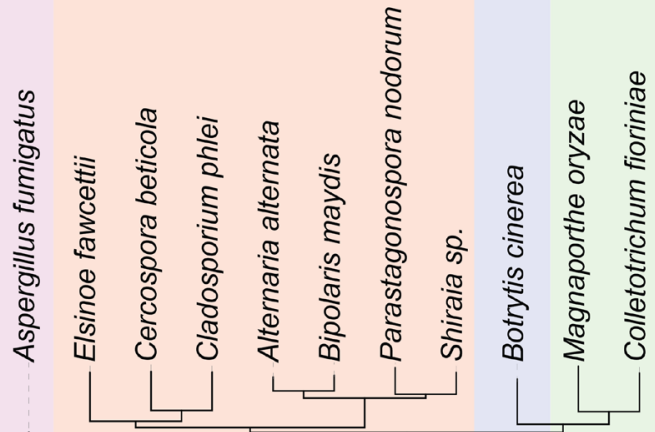

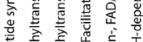

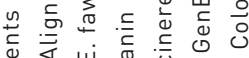

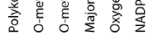

है

का

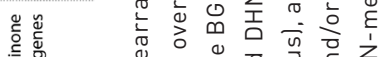

每

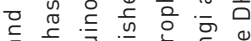

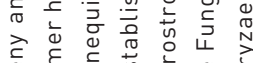

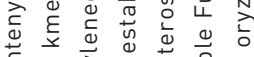

ท)

$\varangle$ 
elsinochrome and phleichrome (Fig. 4A). Overall, eight genes are shared between the cercosporin, hypocrellin and predicted elsinochrome and phleichrome BGCs (Figs. 4A, 5). When compared to these perylenequinone pathways, the CTB gene cluster has two additional genes; a putative a-ketoglutaratedependent dioxygenase (CTB9) and a candidate dehydratase (CTB10) that have been shown to be involved in the formation of the methylenedioxy bridge (13). The predicted C. phlei phleichrome BGC contains all orthologous C. beticola CTB genes except for the above-mentioned CTB9 and CTB10, in agreement with the lack of the methylenedioxy bridge in phleichrome. Likewise, the predicted E. fawcettii elsinochrome BGC lacks CTB9 and CTB10 as well as the cercosporin MFS transporter (CTB4) and the NADPH-dependent oxidoreductase (CTB6). Interestingly, the E. fawcettii BGC contains ELSFAW08009, which only has an ortholog in the hypocrellin gene cluster (SHIR08482) and in no other of the aligned BGCS (Fig. 4A). ELSFAW08009 and SHIR08482 are annotated as a putative salicylate hydroxylase based on sequence similarity to the conserved protein domain family TIGR03219 (E-value 2.98e-18), members of which are salicylate 1-monoxygenases. Besides sharing this gene with the elsinochrome pathway and lacking orthologs to CTB9 and CTB10, the hypocrellin cluster also lacks CTB homologs CTB4, CTB6, and CTB7 compared to the cercosporin pathway (Fig. 4A).

Similarly, predicted DHN-melanin clusters of C. beticola, C. phlei, E. fawcettii, S. bambusicola sp. slf14, and Co. fioriniae were aligned to the established DHN-melanin cluster of M. oryzae, A. fumigatus, A. alternata, Bipolaris maydis (Cochliobolus heterostrophus), and both alternative clusters of B. cinerea (Figure 4B). All BGCs share homologous PKS genes, a THN-reductase, and a prefoldin-encoding gene. Prefoldins are frequently associated with DHN-melanin BGCs, but a functional role in DHN-melanin biosynthesis has not been established to date. Furthermore, the putative melanin clusters of C. beticola, C. phlei, E. fawcettii, S. bambusicola sp. slf14, and Co. fioriniae contain a transcription factor with homology to M. oryzae Pig1 and Co. lagenarium CMR1, which are often observed in other established melanin clusters (40).

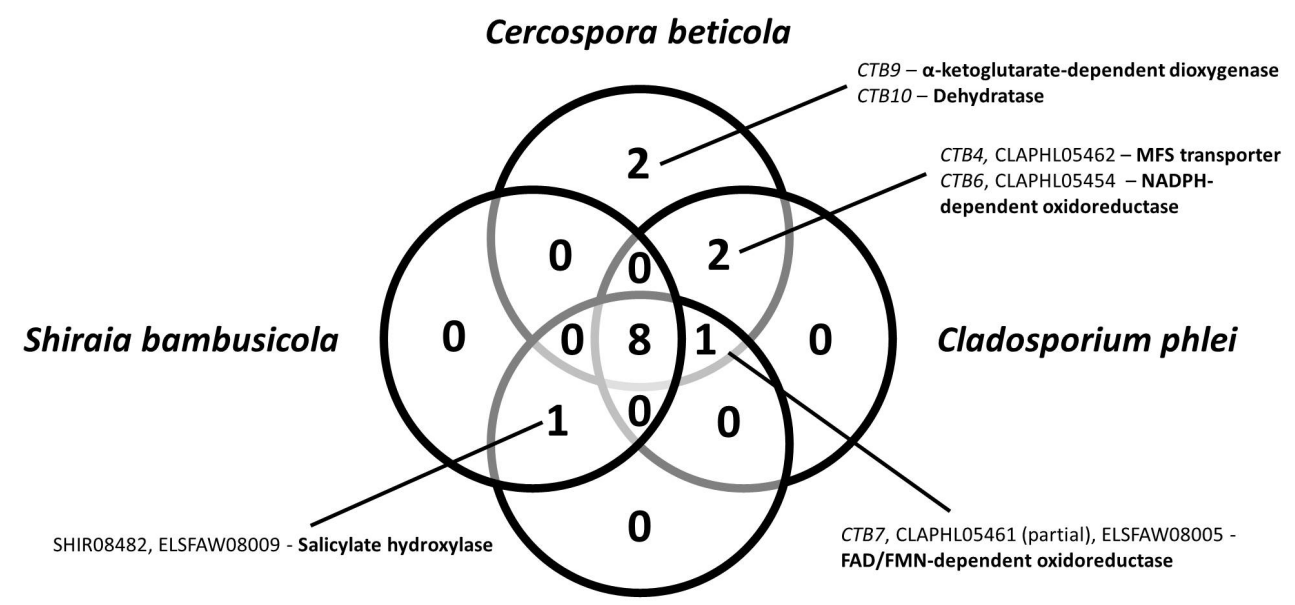

\section{Elsinoë fawcettii}

Figure 5. Conserved and unique genes in the confirmed or predicted perylenequinone BGCs of $C$. beticola, E. fawcettii, C. phlei, and S. bambusicola. Venn diagram highlights the number of shared BGC genes of the cercosporin, elsinochrome, phleichrome, and hypocrellin pathways. 


\section{(A) Cercosporin}

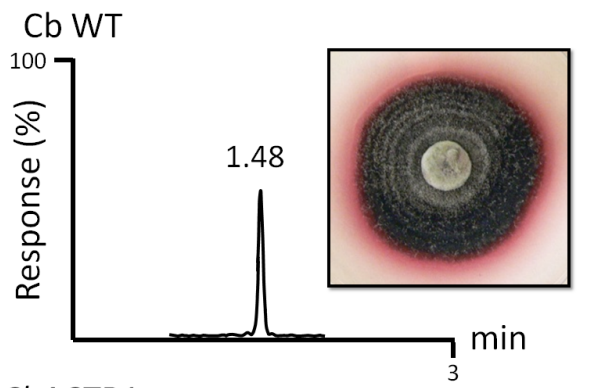

\section{CbDCTB1}

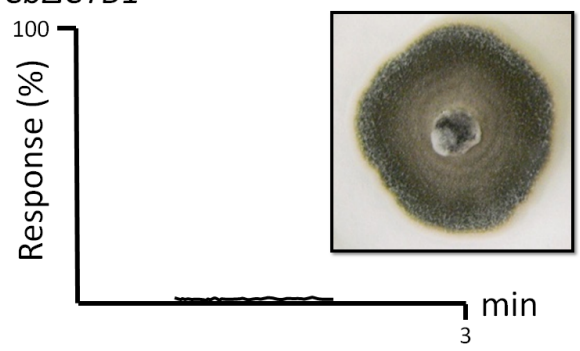

CbDPKS1

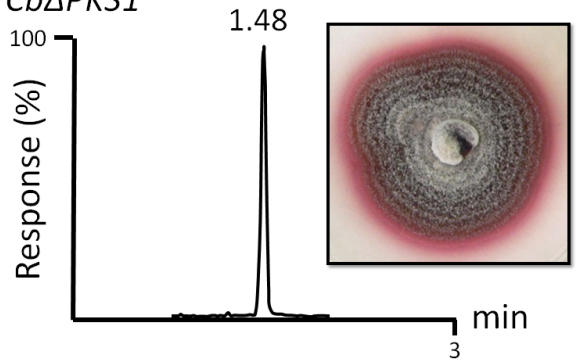

Retention time
(B) Elsinochrome B/D

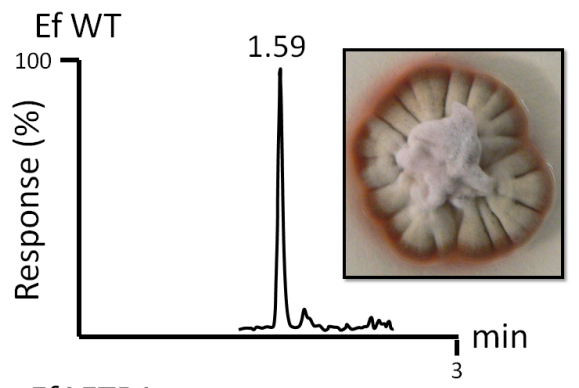

EfDETB1

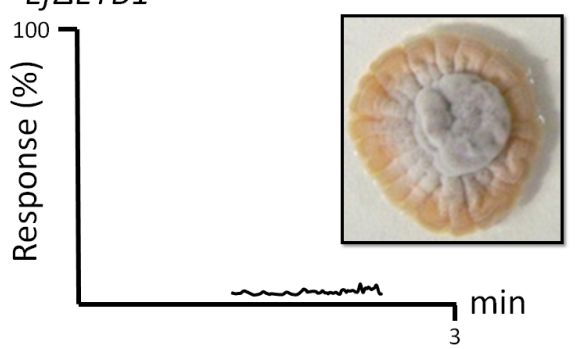

Ef $\triangle P K S 1$

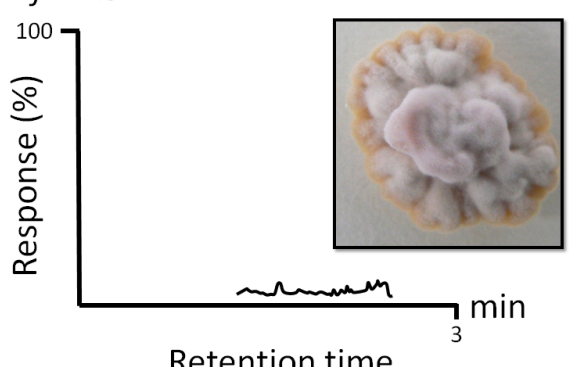

Figure 6. Perylenequinone toxin detection in $C$. beticola and $E$. fawcettii perylenequinone and melanin PKS mutants compared to wild type strains after growth under perylenequinone-inducing conditions. Representative UPLC mass-selective detection of cercosporin (column A) and elsinochrome B/D (column B) are shown for each fungal strain (minimum of 2 plate extracts per strain). Cercosporin (column A) was present in C. beticola wild type and the $C b \triangle P K S 1$ mutants at a (retention time $1.48 \mathrm{~min}$ ) but not in the $C b \triangle C T B 1$ mutants (the cercosporin standard produced a mass-selective chromatogram with an identical retention time; data not shown). An elsinochrome B/D peak (column B) was present only in wild type E. fawcettii strains, retention time $1.59 \mathrm{~min}$, and was undetectable in both Ef $\triangle E T B 1$ and Ef $\triangle P K S 1$ mutants (no chemical-grade standard available).

Targeted replacement and characterization of perylenequinone and melanin PKS genes The predicted perylenequinone and melanin PKS genes for C. beticola and E. fawcettii were targeted for split marker gene replacement. At least two unique site-directed transformants were assessed for involvement in metabolite production. The wild type and knockout mutant strains were grown under conditions to induce perylenequinone production. The presence or absence of cercosporin (C. beticola) and elsinochrome (E. fawcettii) in culture extracts was 
determined via UPLC-MS (Fig. 6). For C. beticola, the production of cercosporin was abrogated in $\triangle C b C T B 1$ mutants but $\triangle C b P K S 1$ mutants were still able to produce cercosporin (Fig. 6A). There were no obvious differences in growth rate for either of the $C$. beticola mutants versus the wild type strain. Additionally, $\triangle$ CbPKS1 mutants had a pale buff color as opposed to the dark grey pigmentation observed in wild type strains (Fig. 6A). For E. fawcettii, both the $\triangle$ EfETB1 and $\triangle E F P K S 1$ mutant strains lacked elsinochrome production whilst the toxin was present as a deep red pigment in the wild type (Fig. 6B). The amount of melanin present in the cultures was determined spectrophotometrically, showing that $\triangle P K S 1$ and $\triangle C T B 1 / \triangle E T B 1$ mutants for both species had a significantly lower melanin content than their respective wild types $(P<0.05)$ (Fig. $7 \mathrm{~A}$ and $\mathrm{B})$. The melanin content of ectopic mutants in both $\mathrm{C}$. beticola and E. fawcettii did not significantly differ from their wild type $(P>0.05)$, demonstrating that the decreased melanin content observed for $\triangle P K S 1, \triangle C T B 1$ and $\triangle E T B 1$ mutants was unlikely an effect of protoplast transformation and was instead caused by the disruption of the targeted gene. The $C$. beticola and E. fawcettii $\triangle P K S 1$ mutants unexpectedly exhibited some melanin extract absorbance at 475 $\mathrm{nm}$, which is likely due to background absorbance.

(A)

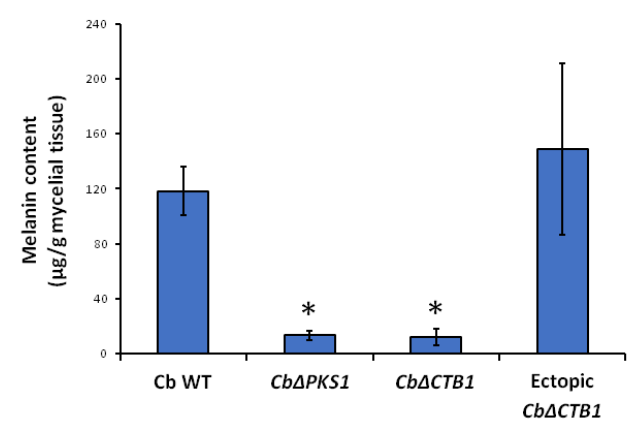

(B)

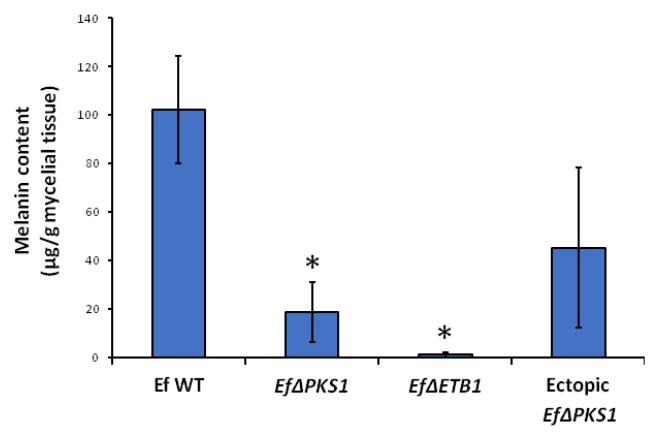

Figure 7. Melanin production in C. beticola and E. fawcettii perylenequinone and melanin PKS mutants compared to wild type strains. The mean melanin content of three individual fungal cultures $(\mu \mathrm{g}$ melanin $/ \mathrm{g}$ of mycelial tissue \pm standard error) in (A) C. beticola wild type (WT), melanin mutants (Cb $\triangle P K S 1$ ), cercosporin

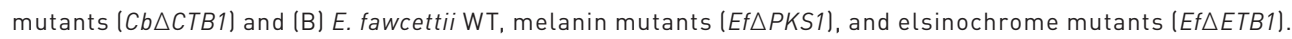
Ectopic mutants were included as positive controls. Significant differences $(P<0.05)$ indicated by *

\section{Discussion}

Phylogenetic analysis based on PKS KS domain conservation can help to predict SM structure and gene evolution (6, 7). In this study, we used KS domain sequence alignments and phylogenetic analysis of selected plant pathogenic fungi to separate PKSs into distinct clades. One of the clades hosted PKS genes involved with perylenequinone biosynthesis including CbCTB1, the well-studied Cercospora beticola PKS essential for cercosporin biosynthesis, and the PKS gene of the hypocrellin pathway in S. bambusicola sp. slf14. We also observed clustering of PKS genes involved in DHN-melanin formation such as Bcpks12 and Bcpks13 of B. cinerea and COGPKS1 of Co. lagenarium (Fig. 3). As previously reported (32), phylogenetic analyses of KS and AT domain 
sequences indicated a closer relationship of EfPKS1 to melanin PKSs than to perylenequinone PKSs. Furthermore, high similarity of the full length amino acid sequence to the annotated EfPKS1 led So et al. (2015) (33) to hypothesize that Cppks1 was involved in phleichrome production. Our KS domain alignment confirms the phylogenetic analysis by Liao et al. (2008) (32) where EfPKS1 and Cppks1 form a cluster with established DHN melanin biosynthesis PKSs of other Ascomycetes (Fig. 3). Consequently, we used comparisons to well-characterized melanin BGCs in various Ascomycetes to show that PKS genes belonging to the DHN-melanin clade are putatively involved with melanin biosynthesis in C. beticola, E. fawcettii, C. phlei, and S. bambusicola sp. slf14 (Fig. 4B). Besides PKS phylogeny, whole-cluster homology of predicted cognate clusters to various well established DHN-melanin clusters strengthened our hypothesis that CbPKS1, EfPKS1, and Cppks1 are involved with melanin production.

To gain further support, we generated PKS mutants in our candidate melanin biosynthesis PKS genes in C. beticola and E. fawcettii. As predicted, the melanin null mutants $\triangle E F P K S 1$ and $\triangle C b P K S 1$ displayed pale phenotypes characteristic to previously described melanin-deficient mutant strains (53) (Fig. 6A and B) and had reduced melanin production (Fig. 7A and B).

Interestingly, the $C$. beticola $\triangle$ CbPKS1 mutant was still able to produce cercosporin, while no elsinochrome production was detected in the E. fawcettii $\triangle E f P K S 1$ mutant. Since elsinochromedeficiency in E. fawcettii $\triangle E F P K S 1$ mutants was also reported by Liao et al. (2008) (32), we suspect that the phenotype observed is a pleiotropic effect. For example, pleiotropic effects of melanin were demonstrated in Rynchosporium commune, where the degree of melanization was positively correlated with both virulence and fungicide resistance (54). It is tempting to speculate that the knock-on effect of melanin production on levels of other secondary metabolites could influence these phenotypes. Toxin and melanin biosynthesis pathways have also been suggested to overlap in Curvularia lunata, where the higher virulence of one strain compared to another was associated with expression differences in both toxin and melanin biosynthesis pathways (55). The reduction in virulence observed for their EfPKS1 deletion mutant (32) is not surprising since melanin has been reported to be a virulence factor for many filamentous fungi (34-36). Besides contribution to fungal virulence, melanin has also been reported to play an important role in protection against environmental stresses. Recently, studies of the causal agent of septoria tritici blotch on wheat, Zymoseptoria tritici, have indicated a correlation between fungicide resistance and melanization level of the producing fungus which led to the identification of the putative Z. tritici melanin PKS $(56,57)$. Similarly, CbPKS1 and CBET3_09636, encoding a predicted tetrahydroxynaphthalene ( $T 4 \mathrm{HN}$ ) reductase (now renamed to Cb4HNR as it is homologous to $4 H N R$ of $M$. oryzae), that we propose to belong to the melanin BGC have been recently reported to be more highly expressed in fungicide-resistant $C$. beticola strains compared to fungicide-sensitive strains (58). Consequently, we propose that melanin production in C. beticola is mediated by CbPKS1 which forms T4HN in the first biosynthetic step. Subsequently, T4HN will serve as substrate for Cb4HNR which reduces it to yield scytalone. Taken together, these results suggest that the EfPKS1 and CPpKs1 genes that were formerly predicted to be involved with elsinochrome and phleichrome biosynthesis were likely incorrectly annotated in previous publications and are involved in DHN-melanin biosynthesis. 
To identify the legitimate elsinochrome and phleichrome PKS genes in E. fawcettii and C. phlei, respectively, we went back to our KS domain alignment where predicted PKSs CPPTB1 of C. phlei and EfETB1 of E. fawcettii clustered together with established cercosporin biosynthesis PKSS CTB1 in C. beticola and C. nicotianae, which hinted at their contribution to perylenequinone biosynthesis (Fig. 3). In line with these initial functional predictions, alignments of the corresponding predicted gene clusters display high similarity and gene conservation within each clade (Fig. 4A). Also, structural differences between perylenequinones can be explained by comparing the predicted metabolite clusters on a gene level. For example, cercosporin and phleichrome only differ in the additional methylenedioxy bridge that is found in the cercosporin molecule (Fig. 1). Accordingly, the predicted phleichrome biosynthesis pathway lacks CTB9 and CTB10 that have been shown to be responsible for methylenedioxy bridge formation (13). Site-directed gene replacement of EfETB1 in E. fawcettii and CbCTB1 in C. beticola led to the successful generation of perylenequinone mutants that are deficient in toxin production under perylenequinone-inducing conditions (Fig. 6A and B). Since SM production relies on different environmental conditions, not every medium is suitable to activate SM production $(59,60)$. For C. beticola, research on cercosporin-inducing conditions resulted in the identification of thin PDA plates in combination with natural light as the induction condition of choice (61-63), which was shown here to stimulate elsinochrome production. Interestingly, we also observed a pleiotropic effect of perylenequinone biosynthesis on melanin content, with the toxin PKS mutants exhibiting reduced melanin levels compared to wild type (Fig. 7A and B).

In conclusion, we have shown that it is possible to identify BGCs of structurally related SM compounds based on the phylogenetic relationship of their encompassing PKSs and overall conservation level of the associated cluster genes. By using an established CTB gene cluster as reference, it was possible to single out gene clusters responsible for the synthesis of related perylenequinone compounds in different fungal species. Likewise, we successfully identified clusters associated with DHN-melanin production in C. beticola, E. fawcettii, C. phlei, P. nodorum, and S. bambusicola using the same approach and the confirmed DHN-melanin cluster as input. Future research using this methodology will be useful for the identification of other perylenequinones and their corresponding BGCs in other fungi.

\section{Materials and Methods}

\section{Elsinoë fawcettii and Cladosporium phlei genome sequencing}

For high-quality genomic DNA extraction of Elsinoë fawcettii strain CBS 139.25 and Cladosporium phlei strain CBS 358.69, mycelia was scraped from the surface of PDA agar petri dishes and extracted using the CTAB method (58). Library preparation (500 bp) and subsequent paired-end (PE) genome sequencing was done by BGI via the Illumina platform. Approximately 34 million high-quality sequence reads with an average length of 100 bp were generated for both samples, representing 134- and 111fold coverage for E. fawcettii and C. phlei respectively. Draft genomes were assembled using SPAdes (version 3.9.0), with default parameters and k-mers 21, 33, 55, 77 and 99. Prediction of protein-coding gene models was performed ab initio using the previously prepared Cercospora beticola training parameters (13) in Augustus (version 3.2.1). Genome sequences and annotations are submitted to $\mathrm{NCBI}$ and permanently linked on figshare under doi https://doi.org/10.6084/m9.figshare.6173834. 


\section{Secondary metabolite phylogenetic analyses}

Phylogenetic analysis of the type I PKS genes and phylogenetic tree analyses were largely performed as described in de Jonge et al. (2018) (13). In short, we used Pfam domain scanning analyses by HMMER3 (64) with hmm profiles for domains PF00109.25 (Betaketoacyl synthase, N-terminal domain) and PF02801.21 (Beta-ketoacyl synthase, C-terminal domain) to identify all PKSs in the predicted proteomes of C. beticola (09-40), C. phlei (CBS 358.69), E. fawcettii (CBS 139.25), S. bambusicola (Slf14), P. nodorum (SN15), C. heterostrophus (C5), A. alternata (SRC1lrk2f), A. fumigatus (Af293), B. cinerea (B05.10), Co. fioriniae (PJ7), and M. oryzae (70-15) that were obtained from NCBI GenBank or Ensemble Fungi. In total we identified 240 proteins across these 11 proteomes. In addition, we added 70 PKSs from Collemare et al. (2014) (48) and Cppks1 (AFP89389.1) from So et al. (2015) (33). All selected proteins for further analyses are listed in Supplementary Table 1. All 311 PKS proteins were subsequently aligned by Mafft (v7.271) using default parameters, after which we extracted the KS domain proportion as previously defined by Pfam scanning. This resulted in an alignment with 311 proteins across 832 positions, that was used to prepare a maximum likelihood phylogenetic tree using RAxML (version 8.2.11), incorporating 100 rapid bootstraps and subsequent automatic, thorough $M L$ search. We then selected the subclass of 94 non-reducing PKSs for further analysis, as defined previously by Kroken et al. (2003) (10). The final phylogenetic tree and figure was prepared in EvolView (65). In this tree, we collapsed the outgroup clade with 20 members containing PKSs involved with citrinin biosynthesis, as indicated in Figure 4. Inclusion in the final set of 74 non-collapsed, nonreducing PKSs is indicated in Supplementary Table 1.

\section{Secondary metabolite cluster alignment visualization}

For comparative analyses of the secondary metabolite clusters across multiple genome sequences we initially identified orthologous protein families across the beforementioned proteomes using orthoFinder (66). Subsequently, we used the MultiGeneBlast algorithm (multigeneblast.sourceforge.net), integral part of antiSMASH (67), to prepare gene-by-gene cluster alignments across all species and we then re-colored individual genes within each gene cluster according to the protein family analysis.

\section{Deletion mutant generation}

Site-directed gene replacements of CTB1 and CDPKS1 in C. beticola strain 1-90 and of EfETB1 and EfPKS1 in E. fawcettii strain CBS 139.25 were generated using the split-marker approach as described in Bolton et al., 2016. Primers are listed in Suppl. Table 2. Regardless of phenotype, all putative knock-out mutants were screened for site-directed gene replacement. Successful gene deletion was confirmed by the presence of a PCR product using a forward primer upstream of the $5^{\prime}$ flanking region of the target gene design and hygromycin reverse primer MDB-1145. Additionally, absence of an amplicon using target gene- specific primers reconfirmed deletion of the target gene (Suppl. Table 2). 


\section{Perylenequinone production assay}

Mycelial plugs of $5 \mathrm{~mm}$ in diameter from PKS mutant and wild type C. beticola and E. fawcettii strains were grown on thin potato dextrose agar (PDA, DifcoTM, BD Diagnostic Systems, Sparks, USA) plates $(3.0 \mathrm{~mL}$ PDA in a $50 \mathrm{~mm}$ Petri plate, amended with $150 \mathrm{\mu g} \mathrm{ml-1}$ hygromycin B (Roche, Mannheim, Germany) (for mutant strains) under a natural light-dark cycle at $21^{\circ} \mathrm{C}$. C. beticola was grown for 7 days and E. fawcettii for 14 days before toxin extraction.

Total mycelial tissue was excised from the agar plate, blended at high speed for $20 \mathrm{~s}$ and extracted with ethyl acetate whilst stirring for 5 min in the dark. Single plate extracts were filtered using two layers of miracloth and dried under a stream of nitrogen $\left(21^{\circ} \mathrm{C}\right)$. The reddish-brown residues were resuspended in $200 \mu \mathrm{l}$ methanol. Cercosporin concentration was calculated by measuring absorbance at $255 \mathrm{~nm}$ using an Agilent Cary 8454 UV-Visible spectrophotometer (Agilent Technologies, Inc., Santa Clara, USA) and 21, 500 as the molar extinction coefficient (68). Extracts were diluted to $\sim 100 \mathrm{pg} \mu \mathrm{l}-1$ with methanol and centrifuged at 3,000 $\times \mathrm{g}$ for 5 min. At a minimum, duplicate plate extracts were submitted for mass spectrometric analyses of each fungal strain.

\section{Mass spectrometric analyses}

Positive mode electrospray ionization settings were optimized for cercosporin by infusing a methanolic cercosporin standard (5 ng/ $\mathrm{LL}$ ) (Sigma; St. Louis, USA) into a Waters (Milford, MA) Acquity triple quadrupole mass spectrometer. The precursor ion, product ions, optimum collision energies, and cone voltage were determined by the AutoTune Wizard within the MassLynx 4.1 software (Waters; Milford, MA). Ion transitions used for cercosporin detection were $\mathrm{m} / \mathrm{z} 535 \rightarrow 415$ and $\mathrm{m} / \mathrm{z} 535 \rightarrow 485$ using a cone voltage of 60 and collision energies of 25 and $20 \mathrm{~V}$, respectively.

Elsinochrome standard was not available, therefore an extract from wild type E. fawcettii was infused into the mass spectrometer and fragmentation of ions appearing at $\mathrm{m} / \mathrm{z} 547$ (the molecular mass of elsinochromes B \& D) were optimized using the AutoTune Wizard within the MassLynx 4.1. Presumptive elsinochrome ion transitions used were $\mathrm{m} / \mathrm{z} 547 \rightarrow 487$ and $\mathrm{m} / \mathrm{z} 547$ $\rightarrow 457$ using a cone voltage of 60 and collision energies of 20 and $35 \mathrm{~V}$, respectively. In some elsinochrome analyses, the mass spectrometer was used as a single sector instrument to collect molecular ions at m/z 547 (elsinochromes B \& D), m/z 545 (elsinochrome A), and m/z 549 (elsinochrome C). For both cercosporin and elsinochrome MS/MS experiments, the desolvation temperature was set at $500{ }^{\circ} \mathrm{C}$, and the source temperature was set at $150{ }^{\circ} \mathrm{C}$. Cone gas (N2) flow was set at $50 \mathrm{~L} / \mathrm{h}$ and desolvation gas flow was set at $800 \mathrm{~L} / \mathrm{h}$, whereas the collision gas (Ar) flow was $0.16 \mathrm{~mL} / \mathrm{min}$.

Cercosporin and elsinochrome (isomers B and D) were analyzed using liquid chromatographytandem mass spectrometry (LC-MS/MS) using a Waters (Milford, MA; USA) Acquity UPLC and Acquity triple-quadrupole mass spectrometer. Data were acquired, processed, and quantified using MassLynx 4.1 with Target Lynx systems. Aliquots of sample extracts (10 $\mu \mathrm{L})$ were injected onto a $2.1 \times 30 \mathrm{~mm}(1.7 \mu \mathrm{m})$ Acquity CSH C18 column protected by a $2.1 \times 5 \mathrm{~mm}$ CHS guard column (Waters; Milford, MA, USA). Cercosporin and elsinochrome were eluted with a binary 
gradient consisting of solvent A $0.1 \%$ formic acid in pure water) and solvent $\mathrm{B}(0.1 \%$ formic acid in acetonitrile) flowing at $1 \mathrm{~mL} / \mathrm{min}$. The gradient program was started at $95 \% \mathrm{~A}$ and transitioned to $25 \%$ A over 2 minutes, 5\% A at 2.1 minutes, and held at 5\% A until 2.5 min when solvent A was ramped back to $95 \%$ A at 3 minutes. Solvent composition was held constant until the end of the run time at $4 \mathrm{~min}$. The column temperature was $30^{\circ} \mathrm{C}$.

\section{Melanin production assay}

Total mycelial tissue from each of wild type and mutant $C$. beticola and E. fawcettii strains was excised from 14 day old cultures (mycelial plugs grown on full strength PDA at $21^{\circ} \mathrm{C}$ with a natural light-dark cycle) and weighed before extracting melanin according to Gadd (1982) (69). The tissue was boiled for $5 \mathrm{~min}$ in $10 \mathrm{~mL}$ distilled water, centrifuged, and the pigment extracted from the supernatant by autoclaving with $3 \mathrm{~mL}$ of $1 \mathrm{M} \mathrm{NaOH}\left(20\right.$ mins, $\left.120^{\circ} \mathrm{C}\right)$. The extract was then acidified to $\mathrm{pH} 2$ with concentrated $\mathrm{HCl}$ to precipitate melanin. The precipitate was washed three times with distilled water and dried under a stream of nitrogen $\left(21^{\circ} \mathrm{C}\right)$.

Melanin extracts were solubilized in $2 \mathrm{~mL}$ of $2 \mathrm{M}$ sodium hydroxide at $50^{\circ} \mathrm{C}$. A spectrophotometric assay was used as described by Kauser et al. (2003) (70) to measure melanin absorbance at $475 \mathrm{~nm}$ with a standard curve of synthetic melanin (Sigma-Aldrich, Milwaukee, USA) from 1-100 $\mathrm{\mu g}$ per $\mathrm{ml}$ to determine melanin content. The mean melanin content was determined as micrograms of melanin per gram of mycelial tissue for three replicates (individual cultures) and the standard error of the mean calculated. Two sample t-tests assuming unequal variances were performed to determine differences between the mean melanin contents of wild type strains and each of the three mutants for C. beticola and E. fawcettii, using a P-value of 0.05 as the significance threshold.

\section{Acknowledgments}

This project was supported in part by USDA-ARS CRIS project 3060-22000-049. Work in the laboratory of B.P.H.J.T. is supported by the Research Council Earth and Life Sciences (ALW) of the Netherlands Organization of Scientific Research (NWO). M.E. was supported by NWO grant 833.13.007. R.d.J. was financially supported by an EMBO Long-Term Fellowship (ALTF 359-2013) and a postdoctoral fellowship of the Research Foundation Flanders (FWO 12B8116N). We thank J. Neubauer (USDA - ARS) for excellent technical assistance. Mention of trade names or commercial products in this publication is solely for the purpose of providing specific information and does not imply recommendation or endorsement by the U.S. Department of Agriculture. 


\section{References}

1. Stergiopoulos I, Collemare J, Mehrabi R, \& De Wit PJ (2012) Phytotoxic secondary metabolites and peptides produced by plant pathogenic Dothideomycete fungi. FEMS microbiology reviews 37(1):67-93.

2. Demain AL \& Fang A (2000) The natural functions of secondary metabolites. History of modern biotechnology i. (Springer), pp 1-39.

3. Williams DH, Stone MJ, Hauck PR, \& Rahman SK (1989) Why are secondary metabolites (natural products) biosynthesized? Journal of Natural Products 52(6):1189-1208.

4. Keller NP (2015) Translating biosynthetic gene clusters into fungal armor and weaponry. Nature chemical biology 11(9):671-677.

5. Rohlfs M \& Churchill ACL (2011) Fungal secondary metabolites as modulators of interactions with insects and other arthropods. Fungal Genetics and Biology 48(1):23-34.

6. Keller NP, Turner G, \& Bennett JW (2005) Fungal secondary metabolism-from biochemistry to genomics. Nature Reviews Microbiology 3(12):937-947.

7. Gallo A, Ferrara M, \& Perrone G (2013) Phylogenetic study of polyketide synthases and nonribosomal peptide synthetases involved in the biosynthesis of mycotoxins. Toxins 5(4):717-742.

8. Brakhage AA (2013) Regulation of fungal secondary metabolism. Nature Reviews Microbiology 11(1):21-32

9. Crawford JM \& Townsend CA (2010) New insights into the formation of fungal aromatic polyketides. Nature Reviews Microbiology 8(12):879-889.

10. Kroken S, Glass NL, Taylor JW, Yoder OC, \& Turgeon BG (2003) Phylogenomic analysis of type I polyketide synthase genes in pathogenic and saprobic ascomycetes. Proceedings of the National Academy of Sciences 100(26):15670-15675.

11. Bohnert M, Wackler B, \& Hoffmeister D (2010) Spotlights on advances in mycotoxin research. Applied Microbiology and Biotechnology 87(1):1-7.

12. Keller NP \& Hohn TM (1997) Metabolic pathway gene clusters in filamentous fungi. Fungal Genetics and Biology 21(1):17-29.

13. de Jonge R, et al. (2018) Gene cluster conservation provides insight into cercosporin biosynthesis and extends production to the genus Colletotrichum. Proceedings of the National Academy of Sciences, doi:10.1073/ pnas.1712798115.

14. Assante G, Locci R, Camarda L, Merlini L, \& Nasini G (1977) Screening of the genus Cercospora for secondary metabolites. Phytochemistry 16(2):243-247.

15. Choquer M, et al. (2005) The CTB1 gene encoding a fungal polyketide synthase is required for cercosporin biosynthesis and fungal virulence of Cercospora nicotianae. Molecular Plant-Microbe Interactions 18(5):468-476.

16. Daub ME \& Ehrenshaft M (2000) The photoactivated Cercospora toxin cercosporin: Contributions to plant disease and fundamental biology. Annual review of phytopathology 38(1):461-490.

17. Callahan TM, Rose MS, Meade MJ, Ehrenshaft M, \& Upchurch RG (1999) CFP, the putative cercosporin transporter of Cercospora kikuchii, is required for wild type cercosporin production, resistance, and virulence on soybean. Molecular Plant-Microbe Interactions 12(10):901-910.

18. Perfect SE, Hughes HB, O'Connell RJ, \& Green JR (1999) Colletotrichum: A model genus for studies on pathology and fungal-plant interactions. Fungal Genetics and Biology 27(2):186-198.

19. Newman AG \& Townsend CA (2016) Molecular characterization of the cercosporin biosynthetic pathway in the fungal plant pathogen Cercospora nicotianae. Journal of the American Chemical Society.

20. Chen H-Q, Lee M-H, \& Chung K-R (2007) Functional characterization of three genes encoding putative oxidoreductases required for cercosporin toxin biosynthesis in the fungus Cercospora nicotianae. Microbiology 153(8):2781-2790.

21. Choquer M, Lee M-H, Bau H-J, \& Chung K-R (2007) Deletion of a MFS transporter-like gene in Cercospora nicotianae reduces cercosporin toxin accumulation and fungal virulence. FEBS Letters 581(3):489-494.

22. Daub ME, Herrero S, \& Chung KR (2013) Reactive oxygen species in plant pathogenesis: The role of perylenequinone photosensitizers. Antioxid Redox Signal 19(9):970-989.

23. Ahonsi MO, Maurhofer M, Boss D, \& Défago G (2005) Relationship between aggressiveness of Stagonospora sp. isolates on field and hedge bindweeds, and in vitro production of fungal metabolites cercosporin, elsinochrome A and leptosphaerodione. European Journal of Plant Pathology 111(3):203-215.

24. Zhenjun D \& Lown JW (1990) Hypocrellins and their use in photosensitization. Photochemistry and Photobiology 52(3):609-616

25. Guedes RC \& Eriksson LA (2007) Photophysics, photochemistry, and reactivity: Molecular aspects of perylenequinone reactions. Photochemical \& Photobiological Sciences 6(10):1089-1096.

26. Foote CS (1976) Photosensitized oxidation and singlet oxygen. Free radicals in biology 2:85-133.

27. Hudson J, Imperial V, Haugland R, \& Diwu Z (1997) Antiviral activities of photoactive perylenequinones. Photochemistry and photobiology 65(2):352-354.

28. Daub ME, Herrero S, \& Chung K-R (2005) Photoactivated perylenequinone toxins in fungal pathogenesis of plants. FEMS Microbiology Letters 252(2):197-206.

29. Weiss U, Merlini L, \& Nasini G (1987) Naturally occurring perylenequinones. Fortschritte der Chemie organischer Naturstoffe / progress in the chemistry of organic natural products, eds Achenbach H, Bhattacharyya P, Chakraborty DP, Goto T, Merlini L, Nasini G, \& Weiss U (Springer Vienna, Vienna), pp 1-71. 
30. Deng H, Gao R, Liao X, \& Cai Y (2017) Genome editing in Shiraia bambusicola using CRISPR-CAS9 system. Journal of Biotechnology 259:228-234

31. Zhao N, et al. (2016) De novo transcriptome assembly in Shiraia bambusicola to investigate putative genes involved in the biosynthesis of hypocrellin A. International Journal of Molecular Sciences 17(3):311.

32. Liao H-L \& Chung K-R (2008) Genetic dissection defines the roles of elsinochrome phytotoxin for fungal pathogenesis and conidiation of the citrus pathogen Elsinoë fawcettii. Molecular Plant-Microbe Interactions 21(4):469-479.

33. So K-K, et al. (2015) Identification of a polyketide synthase gene in the synthesis of phleichrome of the phytopathogenic fungus Cladosporium phlei. Molecules and Cells 38(12):1105-1110.

34. Langfelder K, Streibel M, Jahn B, Haase G, \& Brakhage AA (2003) Biosynthesis of fungal melanins and their importance for human pathogenic fungi. Fungal Genetics and Biology 38(2):143-158.

35. Liu GY \& Nizet $V$ (2009) Color me bad: Microbial pigments as virulence factors. Trends in Microbiology 17(9):406-413

36. Wheeler MH \& Bell AA (1988) Melanins and their importance in pathogenic fungi. Current topics in medical mycology, ed McGinnis MR (Springer New York, New York, NY), pp 338-387.

37. Beltrán-García MJ, et al. (2014) Singlet molecular oxygen generation by light-activated DHN-melanin of the fungal pathogen Mycosphaerella fijiensis in black sigatoka disease of bananas. PLOS ONE 9(3):e91616.

38. Thompson JE, et al. (2000) The second naphthol reductase of fungal melanin biosynthesis in Magnaporthe grisea: Tetrahydroxynaphthalene reductase. Journal of Biological Chemistry 275(45):34867-34872.

39. Talbot NJ (2003) On the trail of a cereal killer: Exploring the biology of Magnaporthe grisea. Annual Review of Microbiology 57(1):177-202.

40. Tsuji G, et al. (2000) Novel fungal transcriptional activators, Cmr1p of Colletotrichum lagenarium and Pig1p of Magnaporthe grisea, contain Cys2His2 zinc finger and zn(II)2cys6 binuclear cluster DNA-binding motifs and regulate transcription of melanin biosynthesis genes in a developmentally specific manner. Molecular Microbiology 38(5):940-954.

41. Oh Y, et al. (2008) Transcriptome analysis reveals new insight into appressorium formation and function in the rice blast fungus Magnaporthe oryzae. Genome Biology 9(5):R85.

42. Pihet M, et al. (2009) Melanin is an essential component for the integrity of the cell wall of Aspergillus fumigatus conidia. BMC Microbiology 9(1):177.

43. Fujii I, et al. (2004) Hydrolytic polyketide shortening by Ayg1p, a novel enzyme involved in fungal melanin biosynthesis. Journal of Biological Chemistry 279(43):44613-44620.

44. Schumacher J (2016) DHN-melanin biosynthesis in the plant pathogenic fungus Botrytis cinerea is based on two developmentally regulated key enzyme (PKS)-encoding genes. Molecular Microbiology 99(4):729-748.

45. Tsai H-F, Wheeler MH, Chang YC, \& Kwon-Chung KJ (1999) A developmentally regulated gene cluster involved in conidial pigment biosynthesis in Aspergillus fumigatus. Journal of Bacteriology 181(20):6469-6477.

46. Stanke M, Diekhans M, Baertsch R, \& Haussler D (2008) Using native and syntenically mapped cDNA alignments to improve de novo gene finding. Bioinformatics 24(5):637-644.

47. Finn RD, et al. (2017) Interpro in 2017-beyond protein family and domain annotations. Nucleic Acids Research 45(D1):D190-D199.

48. Collemare J, Griffiths S, Cox R, \& de Wit P (2014) Secondary metabolism and biotrophic lifestyle in the tomato pathogen Cladosporium fulvum: From comparative genomics to reconstruction of biosynthetic pathways. Book of Abstracts 10th International Mycological Congress, p 41.

49. Feng B, et al. (2001) Molecular cloning and characterization of WdPKS1, a gene involved in dihydroxynaphthalene melanin biosynthesis and virulence in Wangiella (exophiala) dermatitidis. Infection and Immunity 69(3):1781-1794.

50. Zhang A, et al. (2003) Efficient disruption of a polyketide synthase gene (PKS1) required for melanin synthesis through Agrobacterium-mediated transformation of Glarea lozoyensis. Molecular Genetics and Genomics 268(5):645-655.

51. Fulton TR, Ibrahim N, Losada MC, Grzegorski D, \& Tkacz JS (1999) A melanin polyketide synthase (PKS) gene from Nodulisporium sp. That shows homology to the PKS1 gene of Colletotrichum lagenarium. Mol Gen Genet 262(4):714-720

52. Fujii l, et al. (1999) Heterologous expression and product identification of Colletotrichum lagenarium polyketide synthase encoded by the PKS1 gene involved in melanin biosynthesis. Bioscience, Biotechnology, and Biochemistry 63(8):1445-1452.

53. Chumley FG \& Valent B (1990) Genetic analysis of melanin-deficient, nonpathogenic mutants of Magnaporthe grisea. Mol. Plant-Microbe Interact 3(3):135-143

54. Zhu W, Zhan J, \& McDonald BA (2018) Evidence for local adaptation and pleiotropic effects associated with melanization in a plant pathogenic fungus. Fungal Genetics and Biology.

55. Gao S, et al. (2014) Genome sequence and virulence variation-related transcriptome profiles of Curvularia lunata, an important maize pathogenic fungus. BMC Genomics 15(1):627.

56. Lendenmann MH, Croll D, \& MCDonald BA (2015) QTL mapping of fungicide sensitivity reveals novel genes and pleiotropy with melanization in the pathogen Zymoseptoria tritici. Fungal Genetics and Biology 80:53-67.

57. Lendenmann MH, Croll D, Stewart EL, \& McDonald BA (2014) Quantitative trait locus mapping of melanization in the plant pathogenic fungus Zymoseptoria tritici. G3: Genes|Genomes|Genetics 4(12):2519-2533. 
58. Bolton MD, et al. (2016) RNA-sequencing of Cercospora beticola DMI-sensitive and -resistant isolates after treatment with tetraconazole identifies common and contrasting pathway induction. Fungal Genetics and Biology 92:1-13.

59. VanderMolen KM, Raja HA, El-Elimat T, \& Oberlies NH (2013) Evaluation of culture media for the production of secondary metabolites in a natural products screening program. AMB Express 3(1):71.

60. Calvo AM, Wilson RA, Bok JW, \& Keller NP (2002) Relationship between secondary metabolism and fungal development. Microbiology and Molecular Biology Reviews 66(3):447-459.

61. Jenns A, Daub M, \& Upchurch R (1989) Regulation of cercosporin accumulation in culture by medium and temperature manipulation. Phytopathology 79(2):213-219

62. Fajola AO (1978) Cercosporin, a phytotoxin from Cercospora spp. Physiological Plant Pathology 13(2):157-164.

63. Frandsen N (1955) Untersuchungen über Cercospora beticola. Verhalten des Pilzes in künstlicher Kultur. Zucker 8:451-456.

64. Mistry J, Finn RD, Eddy SR, Bateman A, \& Punta M (2013) Challenges in homology search: HMMER3 and convergent evolution of coiled-coil regions. Nucleic Acids Research 41(12):e121-e121.

65. Zhang H, Gao S, Lercher MJ, Hu S, \& Chen W-H (2012) Evolview, an online tool for visualizing, annotating and managing phylogenetic trees. Nucleic Acids Research 40(W1):W569-W572.

66. Emms DM \& Kelly S (2015) Orthofinder: Solving fundamental biases in whole genome comparisons dramatically improves orthogroup inference accuracy. Genome Biology 16(1):157.

67. Weber T, et al. (2015) Antismash 3.0-a comprehensive resource for the genome mining of biosynthetic gene clusters. Nucleic Acids Research 43(W1):W237-W243.

68. Milat M-L \& Blein J-P (1995) Cercospora beticola toxins III. Purification, thin-layer and high performance liquid chromatographic analyses. J Chromatogr A 699(1-2):277-283.

69. Gadd GM (1982) Effects of media composition and light on colony differentiation and melanin synthesis in Microdochium bolleyi. Transactions of the British Mycological Society 78(1):115-122.

70. Kauser S, Schallreuter KU, Thody AJ, Tobin DJ, \& Gummer C (2003) Regulation of human epidermal melanocyte biology by $\beta$-endorphin. Journal of Investigative Dermatology 120(6):1073-1080. 


\section{Supplementary data}

Suppl. Table 1. List of the polyketide synthase (PKS) accession codes used in this study.

\begin{tabular}{|c|c|c|c|c|c|c|}
\hline protein_id & $\begin{array}{c}\text { start } \\
\text { KS } \\
\text { domain }\end{array}$ & $\begin{array}{c}\text { stop } \\
\text { KS } \\
\text { domain }\end{array}$ & Protein ID (Fig. 3) & $\begin{array}{l}\text { NR- } \\
\text { PKS }\end{array}$ & $\begin{array}{l}\text { Collemare_ } \\
\text { etal_2014 }\end{array}$ & Species \\
\hline AAB08104.3 & 12 & 386 & AAB08104.3/12-386 & & 1 & Bipolaris maydis \\
\hline AAC39471.1 & 376 & 751 & AAC39471.1/376-751 & 1 & 1 & Aspergillus fumigatus \\
\hline AAC49191.1 & 381 & 757 & AAC49191.1/381-757 & 1 & 1 & Aspergillus nidulans \\
\hline AAD31436.3 & 367 & 743 & AAD31436.3/367-743 & 1 & 1 & Exophiala dermatitidis \\
\hline AAD34559.1 & 11 & 380 & AAD34559.1/11-380 & & 1 & Aspergillus terreus \\
\hline AAD38786.1 & 383 & 759 & AAD38786.1/383-759 & 1 & 1 & $\begin{array}{l}\text { Nodulisporium sp. } \\
\text { ATCC74245 }\end{array}$ \\
\hline AAK48943.1 & 33 & 406 & AAK48943.1/33-406 & & 1 & Byssochlamys nivea \\
\hline AAN59953.1 & 379 & 754 & AAN59953.1/379-754 & 1 & 1 & Glarea lozoyensis \\
\hline AAR92208.1 & 5 & 382 & AAR92208.1/5-382 & & 1 & Fusarium verticillioides \\
\hline AAS48892.1 & 363 & 738 & AAS48892.1/363-738 & 1 & 1 & Nectria haematococca \\
\hline AAS90093.1 & 372 & 748 & AAS90093.1/372-748 & 1 & 1 & Aspergillus flavus \\
\hline AAS98200.1 & 29 & 402 & AAS98200.1/29-402 & & 1 & Aspergillus ochraceus \\
\hline AAT28740.1 & 10 & 387 & AAT28740.1/10-387 & & 1 & Fusarium verticillioides \\
\hline AАT69682.1 & 382 & 760 & AAT69682.1/382-760 & 1 & 1 & Cercospora nicotianae \\
\hline AAU10633.1 & 380 & 758 & AAU10633.1/380-758 & 1 & 1 & Fusarium graminearum \\
\hline AAX09990.1 & 135 & 712 & AAX09990.1/135-712 & & 1 & Bipolaris maydis \\
\hline ABA02239.1 & 50 & 435 & ABA02239.1/50-435 & & 1 & Monascus pilosus \\
\hline ABA02240.1 & 11 & 380 & ABA02240.1/11-380 & & 1 & Monascus pilosus \\
\hline ABB76806.1 & 11 & 387 & ABB76806.1/11-387 & & 1 & Bipolaris maydis \\
\hline ABB90282.1 & 365 & 738 & ABB90282.1/365-738 & 1 & 1 & Fusarium graminearum \\
\hline ABB90283.1 & 10 & 376 & ABB90283.1/10-376 & & 1 & Fusarium graminearum \\
\hline ABS87601.1 & 9 & 391 & ABS87601.1/9-391 & & 1 & Aspergillus fumigatus Af293 \\
\hline ABU63483.1 & 375 & 751 & ABU63483.1/375-751 & 1 & 1 & Elsinoe fawcettii \\
\hline ACD39762.1 & 366 & 739 & ACD39762.1/366-739 & 1 & 1 & Hypomyces subiculosus \\
\hline ACD39767.1 & 10 & 384 & ACD39767.1/10-384 & & 1 & Hypomyces subiculosus \\
\hline ACS68554.1 & 9 & 389 & ACS68554.1/9-389 & & 1 & Metarhizium anisopliae \\
\hline ACZ57548.1 & 48 & 426 & ACZ57548.1/48-426 & & 1 & Alternaria brassicicola \\
\hline ADY00130.1 & 360 & 725 & ADY00130.1/360-725 & & 1 & Penicillium brevicompactum \\
\hline AFP89389.1 & 370 & 746 & AFP89389.1/370-746 & 1 & & Cladosporium phlei \\
\hline AG086662.1 & 5 & 385 & AG086662.1/5-385 & & 1 & Fusarium heterosporum \\
\hline BAA18956.1 & 382 & 758 & BAA18956.1/382-758 & 1 & 1 & Colletotrichum lagenaria \\
\hline BAC20564.1 & 9 & 394 & BAC20564.1/9-394 & & 1 & Penicillium citrinum \\
\hline BAC20566.1 & 30 & 399 & BAC20566.1/30-399 & & 1 & Penicillium citrinum \\
\hline BAD44749.1 & 383 & 747 & BAD44749.1/383-747 & & 1 & Monascus purpureus \\
\hline
\end{tabular}




\begin{tabular}{|c|c|c|c|c|c|c|}
\hline BAD83684.1 & 3 & 377 & BAD83684.1/3-377 & & 1 & Alternaria solani \\
\hline BAE06845.2 & 146 & 729 & BAE06845.2/146-729 & & 1 & Epichloe festucae \\
\hline BAI43678.1 & 3 & 383 & BAI43678.1/3-383 & & 1 & Aspergillus flavus \\
\hline BAK26562.1 & 3 & 383 & BAK26562.1/3-383 & & 1 & Aspergillus oryzae \\
\hline Bcin01p00060.1 & 6 & 387 & Bcin01p00060.1/6-387 & & & Botrytis cinerea \\
\hline Bcin01p00090.1 & 5 & 377 & Bcin01p00090.1/5-377 & & & Botrytis cinerea \\
\hline Bcin01p00440.1 & 116 & 430 & Bcin01p00440.1/116-430 & & & Botrytis cinerea \\
\hline Bcin01p11550.1 & 7 & 386 & Bcin01p11550.1/7-386 & & & Botrytis cinerea \\
\hline Bcin02p01680.1 & 7 & 379 & Bcin02p01680.1/7-379 & & & Botrytis cinerea \\
\hline Bcin02p08770.1 & 382 & 758 & Bcin02p08770.1/382-758 & 1 & & Botrytis cinerea \\
\hline Bcin02p08830.1 & 394 & 765 & Bcin02p08830.1/394-765 & & & Botrytis cinerea \\
\hline Bcin03p02010.1 & 396 & 761 & Bcin03p02010.1/396-761 & & & Botrytis cinerea \\
\hline Bcin03p04360.1 & 4 & 386 & Bcin03p04360.1/4-386 & & & Botrytis cinerea \\
\hline Bcin03p08050.1 & 373 & 750 & Bcin03p08050.1/373-750 & 1 & & Botrytis cinerea \\
\hline Bcin04p00640.1 & 487 & 857 & Bcin04p00640.1/487-857 & & & Botrytis cinerea \\
\hline Bcin04p06330.1 & 8 & 185 & Bcin04p06330.1/8-185 & & & Botrytis cinerea \\
\hline Bcin05p06220.1 & 360 & 733 & Bcin05p06220.1/360-733 & 1 & & Botrytis cinerea \\
\hline Bcin05p08400.1 & 9 & 383 & Bcin05p08400.1/9-383 & & & Botrytis cinerea \\
\hline Bcin07p02920.1 & 69 & 443 & Bcin07p02920.1/69-443 & & & Botrytis cinerea \\
\hline Bcin08p00290.1 & 34 & 399 & Bcin08p00290.1/34-399 & & & Botrytis cinerea \\
\hline Bcin10p00040.1 & 10 & 401 & Bcin10p00040.1/10-401 & & & Botrytis cinerea \\
\hline Bcin11p02700.1 & 3 & 373 & Bcin11p02700.1/3-373 & & & Botrytis cinerea \\
\hline Bcin13p01510.1 & 7 & 383 & Bcin13p01510.1/7-383 & & & Botrytis cinerea \\
\hline Bcin14p00600.1 & 7 & 381 & Bcin14p00600.1/7-381 & & & Botrytis cinerea \\
\hline Bcin14p01290.1 & 36 & 413 & $B \operatorname{cin} 14 p 01290.1 / 36-413$ & & & Botrytis cinerea \\
\hline Bcin16p01830.1 & 391 & 763 & Bcin16p01830.1/391-763 & 1 & & Botrytis cinerea \\
\hline Bcin16p02410.1 & 2 & 385 & Bcin16p02410.1/2-385 & & & Botrytis cinerea \\
\hline Bcin16p05040.1 & 385 & 757 & Bcin16p05040.1/385-757 & & & Botrytis cinerea \\
\hline CAA46695.2 & 377 & 752 & CAA46695.2/377-752 & 1 & 1 & Aspergillus nidulans \\
\hline CAB92399.1 & 344 & 718 & CAB92399.1/344-718 & 1 & 1 & Fusarium fujikuroi \\
\hline $\begin{array}{l}\text { CADAFUAP } \\
00000040\end{array}$ & 17 & 385 & CADAFUAP00000040/17-385 & & & Aspergillus fumigatus \\
\hline $\begin{array}{l}\text { CADAFUAP } \\
00000167\end{array}$ & 387 & 759 & CADAFUAP00000167/387-759 & & & Aspergillus fumigatus \\
\hline $\begin{array}{l}\text { CADAFUAP } \\
00000313\end{array}$ & 36 & 412 & CADAFUAP00000313/36-412 & & & Aspergillus fumigatus \\
\hline $\begin{array}{l}\text { CADAFUAP } \\
00000361\end{array}$ & 112 & 430 & CADAFUAP00000361/112-430 & & & Aspergillus fumigatus \\
\hline $\begin{array}{l}\text { CADAFUAP } \\
00000765\end{array}$ & 15 & 415 & CADAFUAP00000765/15-415 & & & Aspergillus fumigatus \\
\hline
\end{tabular}




\begin{tabular}{|c|c|c|c|c|c|c|}
\hline $\begin{array}{l}\text { CADAFUAP } \\
00000790\end{array}$ & 386 & 751 & CADAFUAP00000790/386-751 & & & Aspergillus fumigatus \\
\hline $\begin{array}{l}\text { CADAFUAP } \\
00000848\end{array}$ & 9 & 391 & CADAFUAP00000848/9-391 & & & Aspergillus fumigatus \\
\hline $\begin{array}{l}\text { CADAFUAP } \\
00001085\end{array}$ & 390 & 767 & CADAFUAP00001085/390-767 & 1 & & Aspergillus fumigatus \\
\hline $\begin{array}{l}\text { CADAFUAP } \\
00001793\end{array}$ & 5 & 387 & CADAFUAP00001793/5-387 & & & Aspergillus fumigatus \\
\hline $\begin{array}{l}\text { CADAFUAP } \\
00002448\end{array}$ & 392 & 767 & CADAFUAP00002448/392-767 & 1 & & Aspergillus fumigatus \\
\hline $\begin{array}{l}\text { CADAFUAP } \\
00003579\end{array}$ & 2 & 373 & CADAFUAP00003579/2-373 & & & Aspergillus fumigatus \\
\hline $\begin{array}{l}\text { CADAFUAP } \\
00003736\end{array}$ & 11 & 386 & CADAFUAP00003736/11-386 & & & Aspergillus fumigatus \\
\hline $\begin{array}{l}\text { CADAFUAP } \\
00004097\end{array}$ & 376 & 751 & CADAFUAP00004097/376-751 & 1 & & Aspergillus fumigatus \\
\hline $\begin{array}{l}\text { CADAFUAP } \\
00005031\end{array}$ & 67 & 436 & CADAFUAP00005031/67-436 & & & Aspergillus fumigatus \\
\hline $\begin{array}{l}\text { CADAFUAP } \\
00007262\end{array}$ & 10 & 367 & CADAFUAP00007262/10-367 & & & Aspergillus fumigatus \\
\hline $\begin{array}{l}\text { CADAFUAP } \\
00007993\end{array}$ & 408 & 786 & CADAFUAP00007993/408-786 & 1 & & Aspergillus fumigatus \\
\hline $\begin{array}{l}\text { CADAFUAP } \\
00009219\end{array}$ & 50 & 426 & CADAFUAP00009219/50-426 & & & Aspergillus fumigatus \\
\hline CAG28797.1 & 11 & 395 & CAG28797.1/11-395 & & 1 & Magnaporthe grisea \\
\hline CAG28798.1 & 6 & 390 & CAG28798.1/6-390 & & 1 & Magnaporthe grisea \\
\hline CAG29113.1 & 12 & 392 & CAG29113.1/12-392 & & 1 & Magnaporthe oryzae $70-15$ \\
\hline CAL69597.1 & 16 & 401 & CAL69597.1/16-401 & & 1 & Beauveria bassiana \\
\hline CAM35471.1 & 390 & 766 & CAM35471.1/390-766 & 1 & 1 & Sordaria macrospora \\
\hline CA091861.1 & 7 & 386 & CA091861.1/7-386 & & 1 & Penicillium expansum \\
\hline CAP58786.1 & 6 & 387 & CAP58786.1/6-387 & & 1 & Botrytis cinerea \\
\hline CBET3_00098-RA & 382 & 747 & CBET3_00098-RA/382-747 & & & Cercospora beticola \\
\hline CBET3_00833-RA & 383 & 761 & CBET3_00833-RA/383-761 & 1 & & Cercospora beticola \\
\hline CBET3_01483-RA & 6 & 182 & CBET3_01483-RA/6-182 & & & Cercospora beticola \\
\hline CBET3_02338-RA & 401 & 779 & CBET3_02338-RA/401-779 & 1 & & Cercospora beticola \\
\hline CBET3_02473-RA & 385 & 765 & CBET3_02473-RA/385-765 & 1 & & Cercospora beticola \\
\hline CBET3_02934-RA & 4 & 378 & CBET3_02934-RA/4-378 & & & Cercospora beticola \\
\hline CBET3_02994-RA & 371 & 745 & CBET3_02994-RA/371-745 & 1 & & Cercospora beticola \\
\hline CBET3_03238-RA & 31 & 407 & CBET3_03238-RA/31-407 & & & Cercospora beticola \\
\hline CBET3_03942-RA & 1 & 287 & CBET3_03942-RA/1-287 & & & Cercospora beticola \\
\hline
\end{tabular}




\begin{tabular}{|c|c|c|c|c|c|c|}
\hline CBET3_04450-RA & 8 & 182 & CBET3_04450-RA/8-182 & & & Cercospora beticola \\
\hline CBET3_04827-RA & 371 & 747 & CBET3_04827-RA/371-747 & 1 & & Cercospora beticola \\
\hline CBET3_04929-RA & 10 & 188 & CBET3_04929-RA/10-188 & & & Cercospora beticola \\
\hline CBET3_05289-RA & 10 & 383 & CBET3_05289-RA/10-383 & & & Cercospora beticola \\
\hline CBET3_06561-RA & 118 & 436 & CBET3_06561-RA/118-436 & & & Cercospora beticola \\
\hline CBET3_06649-RA & 6 & 383 & CBET3_06649-RA/6-383 & & & Cercospora beticola \\
\hline CBET3_08472-RA & 2 & 370 & CBET3_08472-RA/2-370 & & & Cercospora beticola \\
\hline CBET3_09010-RA & 9 & 381 & CBET3_09010-RA/9-381 & & & Cercospora beticola \\
\hline CBET3_09638-RA & 370 & 746 & CBET3_09638-RA/370-746 & 1 & & Cercospora beticola \\
\hline CBET3_10095-RA & 57 & 424 & CBET3_10095-RA/57-424 & & & Cercospora beticola \\
\hline CBET3_10866-RA & 15 & 381 & CBET3_10866-RA/15-381 & & & Cercospora beticola \\
\hline CBET3_10910-RA & 389 & 765 & CBET3_10910-RA/389-765 & 1 & & Cercospora beticola \\
\hline CBET3_11350-RA & 369 & 743 & CBET3_11350-RA/369-743 & 1 & & Cercospora beticola \\
\hline CBET3_11784-RA & 3 & 389 & CBET3_11784-RA/3-389 & & & Cercospora beticola \\
\hline CBX87032.1 & 5 & 358 & CBX87032.1/5-358 & & 1 & Botrytis cinerea B05.10 \\
\hline CLAPHL03274-RA & 7 & 183 & CLAPHL03274-RA/7-183 & & & Cladosporium phlei \\
\hline CLAPHL04403-RA & 115 & 432 & CLAPHL04403-RA/115-432 & & & Cladosporium phlei \\
\hline CLAPHL04480-RA & 404 & 782 & CLAPHL04480-RA/404-782 & 1 & & Cladosporium phlei \\
\hline CLAPHL05093-RA & 8 & 389 & CLAPHL05093-RA/8-389 & & & Cladosporium phlei \\
\hline CLAPHL05460-RA & 396 & 771 & CLAPHL05460-RA/396-771 & 1 & & Cladosporium phlei \\
\hline CLAPHL05563-RA & 33 & 409 & CLAPHL05563-RA/33-409 & & & Cladosporium phlei \\
\hline CLAPHL05567-RA & 10 & 386 & CLAPHL05567-RA/10-386 & & & Cladosporium phlei \\
\hline CLAPHL08021-RA & 6 & 211 & CLAPHL08021-RA/6-211 & & & Cladosporium phlei \\
\hline CLAPHL08237-RA & 813 & 1194 & CLAPHL08237-RA/813-1194 & & & Cladosporium phlei \\
\hline CLAPHL08786-RA & 370 & 746 & CLAPHL08786-RA/370-746 & 1 & & Cladosporium phlei \\
\hline CLAPHL09453-RA & 6 & 400 & CLAPHL09453-RA/6-400 & & & Cladosporium phlei \\
\hline CLAPHL10557-RA & 1 & 314 & CLAPHL10557-RA/1-314 & & & Cladosporium phlei \\
\hline CLAPHL11044-RA & 39 & 412 & CLAPHL11044-RA/39-412 & & & Cladosporium phlei \\
\hline CLAPHL11108-RA & 12 & 358 & CLAPHL11108-RA/12-358 & & & Cladosporium phlei \\
\hline EAA65602.1 & 436 & 812 & EAA65602.1/436-812 & & 1 & $\begin{array}{l}\text { Aspergillus nidulans FGSC } \\
\text { A4 }\end{array}$ \\
\hline EAA65604.1 & 13 & 359 & EAA65604.1/13-359 & & 1 & $\begin{array}{l}\text { Aspergillus nidulans FGSC } \\
\text { A4 }\end{array}$ \\
\hline EAT76544.2 & 1 & 257 & EAT76544.2/1-257 & & & ParaStagonospora nodorum \\
\hline EAT76667.2 & 166 & 542 & EAT76667.2/166-542 & 1 & & ParaStagonospora nodorum \\
\hline EAT77779.2 & 6 & 374 & EAT77779.2/6-374 & & & ParaStagonospora nodorum \\
\hline EAT79399.1 & 6 & 184 & EAT79399.1/6-184 & & & ParaStagonospora nodorum \\
\hline EAT79697.2 & 1 & 163 & EAT79697.2/1-163 & & & ParaStagonospora nodorum \\
\hline EAT80393.2 & 368 & 725 & EAT80393.2/368-725 & 1 & & ParaStagonospora nodorum \\
\hline EAT80980.2 & 19 & 320 & EAT80980.2/19-320 & & & ParaStagonospora nodorum \\
\hline EAT81575.2 & 80 & 452 & EAT81575.2/80-452 & & & ParaStagonospora nodorum \\
\hline
\end{tabular}




\begin{tabular}{|c|c|c|c|c|c|c|}
\hline EAT82267.2 & 110 & 486 & EAT82267.2/110-486 & 1 & & ParaStagonospora nodorum \\
\hline EAT82755.2 & 1 & 262 & EAT82755.2/1-262 & & & ParaStagonospora nodorum \\
\hline EAT82888.2 & 51 & 420 & EAT82888.2/51-420 & & & ParaStagonospora nodorum \\
\hline EAT83281.2 & 59 & 371 & EAT83281.2/59-371 & & & ParaStagonospora nodorum \\
\hline EAT83782.2 & 166 & 548 & EAT83782.2/166-548 & 1 & & ParaStagonospora nodorum \\
\hline EAT84550.2 & 20 & 397 & EAT84550.2/20-397 & 1 & & ParaStagonospora nodorum \\
\hline EAT85332.2 & 9 & 362 & EAT85332.2/9-362 & & & ParaStagonospora nodorum \\
\hline EAT85671.2 & 333 & 703 & EAT85671.2/333-703 & & & ParaStagonospora nodorum \\
\hline EAT86513.1 & 345 & 666 & EAT86513.1/345-666 & & & ParaStagonospora nodorum \\
\hline EAT87259.2 & 5 & 369 & EAT87259.2/5-369 & & & ParaStagonospora nodorum \\
\hline EAT87501.1 & 6 & 184 & EAT87501.1/6-184 & & & ParaStagonospora nodorum \\
\hline EAT89292.2 & 1 & 171 & EAT89292.2/1-171 & & & ParaStagonospora nodorum \\
\hline EAT90378.1 & 116 & 432 & EAT90378.1/116-432 & & & ParaStagonospora nodorum \\
\hline EAT91803.2 & 11 & 358 & EAT91803.2/11-358 & & & ParaStagonospora nodorum \\
\hline EAT91972.1 & 1 & 348 & EAT91972.1/1-348 & & & ParaStagonospora nodorum \\
\hline EAT92602.2 & 75 & 388 & EAT92602.2/75-388 & & & ParaStagonospora nodorum \\
\hline EAU38971.1 & 5 & 385 & EAU38971.1/5-385 & & 1 & $\begin{array}{l}\text { Aspergillus terreus } \\
\text { NIH } 2624\end{array}$ \\
\hline EEP78969.1 & 12 & 394 & EEP78969.1/12-394 & & 1 & Uncinocarpus reesii 1704 \\
\hline EGU88865.1 & 5 & 382 & EGU88865.1/5-382 & & 1 & Fusarium oxysporum Fo5176 \\
\hline EHA28527.1 & 376 & 751 & EHA28527.1/376-751 & 1 & 1 & Aspergillus niger ATCC 1015 \\
\hline ELSFAW00772-RA & 118 & 435 & ELSFAW00772-RA/118-435 & & & Elsinoë fawcettii \\
\hline ELSFAW04358-RA & 3 & 388 & ELSFAW04358-RA/3-388 & & & Elsinoë fawcettii \\
\hline ELSFAW04473-RA & 6 & 211 & ELSFAW04473-RA/6-211 & & & Elsinoë fawcettii \\
\hline ELSFAW04998-RA & 6 & 380 & ELSFAW04998-RA/6-380 & & & Elsinoë fawcettii \\
\hline ELSFAW05017-RA & 85 & 451 & ELSFAW05017-RA/85-451 & & & Elsinoë fawcettii \\
\hline ELSFAW06585-RA & 1 & 294 & ELSFAW06585-RA/1-294 & & & Elsinoë fawcettii \\
\hline ELSFAW07673-RA & 380 & 758 & ELSFAW07673-RA/380-758 & 1 & & Elsinoë fawcettii \\
\hline ELSFAW08003-RA & 382 & 761 & ELSFAW08003-RA/382-761 & 1 & & Elsinoë fawcettii \\
\hline ELSFAW09157-RA & 375 & 751 & ELSFAW09157-RA/375-751 & 1 & & Elsinoë fawcettii \\
\hline EMD84807 & 3 & 377 & EMD84807/3-377 & & & Cochliobolus heterostrophus \\
\hline EMD84907 & 3 & 377 & EMD84907/3-377 & & & Cochliobolus heterostrophus \\
\hline EMD85328 & 213 & 582 & EMD85328/213-582 & & & Cochliobolus heterostrophus \\
\hline EMD85570 & 25 & 402 & EMD85570/25-402 & & & Cochliobolus heterostrophus \\
\hline EMD85852 & 2 & 379 & EMD85852/2-379 & & & Cochliobolus heterostrophus \\
\hline EMD87534 & 33 & 404 & EMD87534/33-404 & & & Cochliobolus heterostrophus \\
\hline EMD88374 & 6 & 377 & EMD88374/6-377 & & & Cochliobolus heterostrophus \\
\hline EMD88531 & 7 & 185 & EMD88531/7-185 & & & Cochliobolus heterostrophus \\
\hline EMD88582 & 70 & 441 & EMD88582/70-441 & & & Cochliobolus heterostrophus \\
\hline EMD89014 & 112 & 426 & EMD89014/112-426 & & & Cochliobolus heterostrophus \\
\hline EMD89247 & 7 & 351 & EMD89247/7-351 & & & Cochliobolus heterostrophus \\
\hline
\end{tabular}




\begin{tabular}{|c|c|c|c|c|c|c|}
\hline EMD89515 & 15 & 358 & EMD89515/15-358 & 1 & & Cochliobolus heterostrophus \\
\hline EMD90232 & 21 & 395 & EMD90232/21-395 & & & Cochliobolus heterostrophus \\
\hline EMD90816 & 6 & 383 & EMD90816/6-383 & & & Cochliobolus heterostrophus \\
\hline EMD91115 & 13 & 385 & EMD91115/13-385 & & & Cochliobolus heterostrophus \\
\hline EMD92722 & 2 & 379 & EMD92722/2-379 & & & Cochliobolus heterostrophus \\
\hline EMD93030 & 19 & 389 & EMD93030/19-389 & & & Cochliobolus heterostrophus \\
\hline EMD93080 & 7 & 377 & EMD93080/7-377 & & & Cochliobolus heterostrophus \\
\hline EMD93081 & 375 & 755 & EMD93081/375-755 & & & Cochliobolus heterostrophus \\
\hline EMD93171 & 9 & 388 & EMD93171/9-388 & & & Cochliobolus heterostrophus \\
\hline EMD93898 & 388 & 767 & EMD93898/388-767 & 1 & & Cochliobolus heterostrophus \\
\hline EMD94543 & 459 & 827 & EMD94543/459-827 & & & Cochliobolus heterostrophus \\
\hline EMD95112 & 3 & 377 & EMD95112/3-377 & & & Cochliobolus heterostrophus \\
\hline EMD96875 & 371 & 747 & EMD96875/371-747 & 1 & & Cochliobolus heterostrophus \\
\hline EMD97689 & 6 & 184 & EMD97689/6-184 & & & Cochliobolus heterostrophus \\
\hline EMD97890 & 18 & 388 & EMD97890/18-388 & & & Cochliobolus heterostrophus \\
\hline EMD97899 & 649 & 1023 & EMD97899/649-1023 & & & Cochliobolus heterostrophus \\
\hline EME39092.1 & 373 & 749 & EME39092.1/373-749 & 1 & 1 & $\begin{array}{l}\text { Dothistroma septosporum } \\
\text { NZE10 }\end{array}$ \\
\hline EXF73224 & 7 & 387 & EXF73224/7-387 & & & Colletotrichum fioriniae \\
\hline EXF74064 & 5 & 385 & EXF74064/5-385 & & & Colletotrichum fioriniae \\
\hline EXF75718 & 7 & 386 & EXF75718/7-386 & & & Colletotrichum fioriniae \\
\hline EXF75878 & 50 & 428 & EXF75878/50-428 & & & Colletotrichum fioriniae \\
\hline EXF76094 & 2 & 376 & EXF76094/2-376 & & & Colletotrichum fioriniae \\
\hline EXF76712 & 391 & 769 & EXF76712/391-769 & 1 & & Colletotrichum fioriniae \\
\hline EXF77318 & 7 & 401 & EXF77318/7-401 & & & Colletotrichum fioriniae \\
\hline EXF77645 & 5 & 376 & EXF77645/5-376 & & & Colletotrichum fioriniae \\
\hline EXF77657 & 18 & 388 & EXF77657/18-388 & & & Colletotrichum fioriniae \\
\hline EXF77788 & 16 & 383 & EXF77788/16-383 & & & Colletotrichum fioriniae \\
\hline EXF77798 & 116 & 434 & EXF77798/116-434 & & & Colletotrichum fioriniae \\
\hline EXF77954 & 7 & 309 & EXF77954/7-309 & & & Colletotrichum fioriniae \\
\hline EXF78137 & 202 & 572 & EXF78137/202-572 & 1 & & Colletotrichum fioriniae \\
\hline EXF79058 & 3 & 377 & EXF79058/3-377 & & & Colletotrichum fioriniae \\
\hline EXF79508 & 8 & 290 & EXF79508/8-290 & & & Colletotrichum fioriniae \\
\hline EXF79511 & 30 & 404 & EXF79511/30-404 & & & Colletotrichum fioriniae \\
\hline EXF79648 & 17 & 381 & EXF79648/17-381 & & & Colletotrichum fioriniae \\
\hline EXF80059 & 382 & 758 & EXF80059/382-758 & 1 & & Colletotrichum fioriniae \\
\hline EXF80071 & 11 & 383 & EXF80071/11-383 & & & Colletotrichum fioriniae \\
\hline EXF80297 & 9 & 382 & EXF80297/9-382 & & & Colletotrichum fioriniae \\
\hline EXF80380 & 2 & 384 & EXF80380/2-384 & & & Colletotrichum fioriniae \\
\hline EXF80608 & 15 & 389 & EXF80608/15-389 & & & Colletotrichum fioriniae \\
\hline EXF84093 & 400 & 780 & EXF84093/400-780 & 1 & & Colletotrichum fioriniae \\
\hline
\end{tabular}




\begin{tabular}{|c|c|c|c|c|c|c|}
\hline EXF85208 & 17 & 403 & EXF85208/17-403 & & & Colletotrichum fioriniae \\
\hline EXF85213 & 58 & 410 & EXF85213/58-410 & & & Colletotrichum fioriniae \\
\hline EXF85322 & 10 & 394 & EXF85322/10-394 & & & Colletotrichum fioriniae \\
\hline EXF85385 & 90 & 464 & EXF85385/90-464 & & & Colletotrichum fioriniae \\
\hline EXF85533 & 10 & 188 & EXF85533/10-188 & & & Colletotrichum fioriniae \\
\hline EXF86307 & 367 & 742 & EXF86307/367-742 & 1 & & Colletotrichum fioriniae \\
\hline EXF86315 & 10 & 384 & EXF86315/10-384 & & & Colletotrichum fioriniae \\
\hline ACB12550.1 & 30 & 401 & ACB12550.1/30-401 & & 1 & Fusarium oxysporum \\
\hline MGG_00233T0 & 11 & 384 & MGG_00233T0/11-384 & & & Magnaporthe oryzae \\
\hline MGG_00241T0 & 380 & 751 & MGG_00241T0/380-751 & 1 & & Magnaporthe oryzae \\
\hline MGG_00428T0 & 412 & 789 & MGG_00428T0/412-789 & 1 & & Magnaporthe oryzae \\
\hline MGG_00806T0 & 156 & 443 & MGG_00806T0/156-443 & & & Magnaporthe oryzae \\
\hline MGG_03810T0 & 12 & 395 & MGG_03810T0/12-395 & & & Magnaporthe oryzae \\
\hline MGG_04775T0 & 62 & 333 & MGG_04775T0/62-333 & & & Magnaporthe oryzae \\
\hline MGG_05589T0 & 66 & 441 & MGG_05589T0/66-441 & & & Magnaporthe oryzae \\
\hline MGG_07219T0 & 388 & 764 & MGG_07219T0/388-764 & 1 & & Magnaporthe oryzae \\
\hline MGG_07803T0 & 1263 & 1660 & MGG_07803T0/1263-1660 & & & Magnaporthe oryzae \\
\hline MGG_08236T0 & 7 & 380 & MGG_08236T0/7-380 & & & Magnaporthe oryzae \\
\hline MGG_08281T0 & 46 & 470 & MGG_08281T0/46-470 & 1 & & Magnaporthe oryzae \\
\hline MGG_08285T0 & 12 & 364 & MGG_08285T0/12-364 & & & Magnaporthe oryzae \\
\hline MGG_09589T0 & 3 & 412 & MGG_09589T0/3-412 & & & Magnaporthe oryzae \\
\hline MGG_09645T0 & 51 & 415 & MGG_09645T0/51-415 & & & Magnaporthe oryzae \\
\hline MGG_10011T0 & 404 & 776 & MGG_10011T0/404-776 & & & Magnaporthe oryzae \\
\hline MGG_10202T0 & 4 & 407 & MGG_10202T0/4-407 & & & Magnaporthe oryzae \\
\hline MGG_10912T0 & 52 & 425 & MGG_10912T0/52-425 & & & Magnaporthe oryzae \\
\hline MGG_11638T0 & 9 & 395 & MGG_11638T0/9-395 & & & Magnaporthe oryzae \\
\hline MGG_12154T0 & 116 & 433 & MGG_12154T0/116-433 & & & Magnaporthe oryzae \\
\hline MGG_12214T0 & 7 & 378 & MGG_12214T0/7-378 & & & Magnaporthe oryzae \\
\hline MGG_12447T0 & 11 & 395 & MGG_12447T0/11-395 & & & Magnaporthe oryzae \\
\hline MGG_12613T0 & 19 & 385 & MGG_12613T0/19-385 & & & Magnaporthe oryzae \\
\hline MGG_13591T0 & 1 & 311 & MGG_13591T0/1-311 & & & Magnaporthe oryzae \\
\hline MGG_13767T0 & 9 & 388 & MGG_13767T0/9-388 & & & Magnaporthe oryzae \\
\hline MGG_14831T0 & 36 & 410 & MGG_14831T0/36-410 & & & Magnaporthe oryzae \\
\hline MGG_14897T0 & 2 & 391 & MGG_14897T0/2-391 & & & Magnaporthe oryzae \\
\hline MGG_14943T0 & 9 & 366 & MGG_14943T0/9-366 & & & Magnaporthe oryzae \\
\hline MGG_14945T0 & 107 & 481 & MGG_14945T0/107-481 & & & Magnaporthe oryzae \\
\hline MGG_15097T0 & 6 & 390 & MGG_15097T0/6-390 & & & Magnaporthe oryzae \\
\hline MGG_15100T0 & 12 & 393 & MGG_15100T0/12-393 & & & Magnaporthe oryzae \\
\hline MGG_15272T0 & 4 & 381 & MGG_15272T0/4-381 & & & Magnaporthe oryzae \\
\hline MGG_18133T0 & 1 & 314 & MGG_18133T0/1-314 & 1 & & Magnaporthe oryzae \\
\hline OAG13303 & 19 & 397 & OAG13303/19-397 & & & Alternaria alternata \\
\hline
\end{tabular}




\begin{tabular}{|c|c|c|c|c|c|c|}
\hline OAG13655 & 47 & 418 & OAG13655/47-418 & & & Alternaria alternata \\
\hline OAG13710 & 7 & 185 & OAG13710/7-185 & & & Alternaria alternata \\
\hline OAG14483 & 7 & 382 & OAG14483/7-382 & & & Alternaria alternata \\
\hline OAG15814 & 1 & 326 & OAG15814/1-326 & & & Alternaria alternata \\
\hline OAG16734 & 9 & 374 & OAG16734/9-374 & & & Alternaria alternata \\
\hline OAG17698 & 1129 & 1501 & OAG17698/1129-1501 & & & Alternaria alternata \\
\hline OAG18296 & 6 & 184 & OAG18296/6-184 & & & Alternaria alternata \\
\hline OAG18885 & 2 & 376 & OAG18885/2-376 & & & Alternaria alternata \\
\hline OAG18929 & 5 & 383 & OAG18929/5-383 & & & Alternaria alternata \\
\hline OAG22978 & 21 & 395 & OAG22978/21-395 & & & Alternaria alternata \\
\hline OAG24024 & 116 & 430 & OAG24024/116-430 & & & Alternaria alternata \\
\hline OAG24502 & 371 & 747 & OAG24502/371-747 & 1 & & Alternaria alternata \\
\hline OAG24819 & 139 & 515 & OAG24819/139-515 & 1 & & Alternaria alternata \\
\hline OAG25652 & 259 & 629 & OAG25652/259-629 & & & Alternaria alternata \\
\hline OAG26059 & 2 & 382 & OAG26059/2-382 & & & Alternaria alternata \\
\hline P22367.1 & 33 & 406 & P22367.1/33-406 & & 1 & Penicillium griseofulvum \\
\hline Q9Y8A5.1 & 9 & 394 & Q9Y8A5.1/9-394 & & 1 & Aspergillus terreus \\
\hline SHIR00028-RA & 14 & 394 & SHIR00028-RA/14-394 & & & Shiraia sp. \\
\hline SHIR00389-RA & 390 & 757 & SHIR00389-RA/390-757 & & & Shiraia sp. \\
\hline SHIR00708-RA & 53 & 427 & SHIR00708-RA/53-427 & & & Shiraia sp. \\
\hline SHIR01733-RA & 14 & 394 & SHIR01733-RA/14-394 & & & Shiraia sp. \\
\hline SHIR02320-RA & 372 & 748 & SHIR02320-RA/372-748 & 1 & & Shiraia sp. \\
\hline SHIR02347-RA & 36 & 409 & SHIR02347-RA/36-409 & & & Shiraia sp. \\
\hline SHIR02657-RA & 14 & 337 & SHIR02657-RA/14-337 & & & Shiraia sp. \\
\hline SHIR02743-RA & 55 & 432 & SHIR02743-RA/55-432 & & & Shiraia sp. \\
\hline SHIR03412-RA & 1 & 158 & SHIR03412-RA/1-158 & & & Shiraia sp. \\
\hline SHIR04032-RA & 116 & 434 & SHIR04032-RA/116-434 & & & Shiraia sp. \\
\hline SHIR05834-RA & 9 & 383 & SHIR05834-RA/9-383 & & & Shiraia sp. \\
\hline SHIR05992-RA & 259 & 636 & SHIR05992-RA/259-636 & 1 & & Shiraia sp. \\
\hline SHIR06023-RA & 11 & 393 & SHIR06023-RA/11-393 & & & Shiraia sp. \\
\hline SHIR06623-RA & 367 & 745 & SHIR06623-RA/367-745 & 1 & & Shiraia sp. \\
\hline SHIR06625-RA & 1 & 286 & SHIR06625-RA/1-286 & & & Shiraia sp. \\
\hline SHIR06679-RA & 372 & 747 & SHIR06679-RA/372-747 & 1 & & Shiraia sp. \\
\hline SHIR08141-RA & 14 & 392 & SHIR08141-RA/14-392 & & & Shiraia sp. \\
\hline SHIR08147-RA & 394 & 769 & SHIR08147-RA/394-769 & 1 & & Shiraia sp. \\
\hline SHIR08477-RA & 373 & 755 & SHIR08477-RA/373-755 & 1 & & Shiraia sp. \\
\hline SHIR08754-RA & 396 & 774 & SHIR08754-RA/396-774 & 1 & & Shiraia sp. \\
\hline SHIR08896-RA & 112 & 486 & SHIR08896-RA/112-486 & & & Shiraia sp. \\
\hline SHIR09115-RA & 7 & 185 & SHIR09115-RA/7-185 & & & Shiraia sp. \\
\hline SHIR10307-RA & 12 & 387 & SHIR10307-RA/12-387 & & & Shiraia sp. \\
\hline SHIR10308-RA & 383 & 756 & SHIR10308-RA/383-756 & 1 & & Shiraia sp. \\
\hline
\end{tabular}




\begin{tabular}{|l|c|c|l|c|c|l|}
\hline XP_001217072.1 & 403 & 781 & XP_001217072.1/403-781 & 1 & 1 & Aspergillus terreus NIH2624 \\
\hline XP_001221381.1 & 14 & 396 & XP_001221381.1/14-396 & & 1 & $\begin{array}{l}\text { Chaetomium globosum } \\
\text { CBS 148.51 }\end{array}$ \\
\hline XP_001242733.1 & 11 & 387 & XP_001242733.1/11-387 & & 1 & Coccidioides immitis RS \\
\hline XP_001269050.1 & 5 & 383 & XP_001269050.1/5-383 & & 1 & Aspergillus clavatus NRRL 1 \\
\hline XP_001270543.1 & 9 & 393 & XP_001270543.1/9-393 & & 1 & Aspergillus clavatus NRRL 1 \\
\hline XP_001790998.1 & 11 & 358 & XP_001790998.1/11-358 & & 1 & Phaeosphaeria nodorum SN15 \\
\hline XP_001910795.1 & 382 & 758 & XP_001910795.1/382-758 & 1 & 1 & Podospora anserina S mat+ \\
\hline XP_003715434.1 & 388 & 764 & XP_003715434.1/388-764 & 1 & 1 & Magnaporthe oryzae 70-15 \\
\hline XP_390640.1 & 7 & 366 & XP_390640.1/7-366 & & 1 & Fusarium graminearum PH-1 \\
\hline XP_657754.1 & 417 & 795 & XP_657754.1/417-795 & 1 & 1 & Aspergillus nidulans FGSC A4 \\
\hline XP_681681.1 & 3 & 388 & XP_681681.1/3-388 & & 1 & Aspergillus nidulans FGSC A4 \\
\hline XP_746435.1 & 392 & 767 & XP_746435.1/392-767 & 1 & 1 & Aspergillus fumigatus Af293 \\
\hline XP_748662.1 & 138 & 723 & XP_748662.1/138-723 & & 1 & Aspergillus fumigatus Af293 \\
\hline
\end{tabular}

Supl. Table 2. Primers used in this study

\begin{tabular}{|c|c|c|}
\hline Primer & Specificity/role & Sequence $\left(5^{\prime}\right.$ to $\left.3^{\prime}\right)$ \\
\hline MDB-277 & Split-marker M13F (HYG-F) & GACGTTGTAAAACGACGGCCAGTG \\
\hline MDB-258 & Split-marker: HY (NLC37) (HY-R) & GGATGCCTCCGCTCGAAGTA \\
\hline MDB-259 & Split-marker: YG (NLC38) (YG-F) & CGTTGCAAGACCTGCCTGAA \\
\hline MDB-278 & Split-marker M13R (HYG-R) & CACAGGAAACAGCTATGACCATGA \\
\hline MDB-1145 & HY-R2 (split marker) & GGCAGGTAGATGACGACCAT \\
\hline MDB-1598 & Ef ELS PKS 1F & CGAACCGAGCAACAGTGATA \\
\hline MDB-1599 & Ef ELS PKS 2R & CACTGGCCGTCGTTTTACAACGTCACGGAGATTCTGGCTGCTTA \\
\hline MDB-1600 & Ef ELS PKS 3F & TCATGGTCATAGCTGTTTCCTGTGCACCTGGTAGAAGGCGCTAC \\
\hline MDB-1601 & Ef ELS PKS 4R & TCAACATGCTGACAGATTGC \\
\hline MDB-1602 & Ef ELS PKS 5’1F & GGAGTCCAGAGATCCGACTG \\
\hline MDB-1603 & Ef MEL PKS 1F & ACGCTGCACATGTTATCGAG \\
\hline MDB-1604 & Ef MEL PKS 2R & CACTGGCCGTCGTTTTACAACGTCCTTCTTGACGGGGTATCGAA \\
\hline MDB-1605 & Ef MEL PKS 3F & TCATGGTCATAGCTGTTTCCTGTGGGAGTCGAGAGGGAAAGGTC \\
\hline MDB-1606 & Ef MEL PKS 4R & GCCATGTAGAGGAGGTGGAA \\
\hline MDB-1607 & Ef MEL PKS 5’1F & GTCACGTCGAGTCCACACAC \\
\hline MKE-177 & Ef Mel gene spec Fp & TGGGTACAACGTGGCTCATA \\
\hline MKE-178 & Ef Mel gene spec Rp & GACGATGAAGCCACCAAGAT \\
\hline MKE-179 & Ef Els gene spec Fp & CAAGGAACAAATGCAGAGCA \\
\hline MKE-180 & Ef Els gene spec Rp & GAGCCGACTCAAAATCCTTG \\
\hline MDB-1444 & Cb CTB1 1F & TCCTCTGGTGCTATGTCACG \\
\hline MDB-1445 & Cb СТВ1 2R & CACTGGCCGTCGTTTTACAACGTCGAGATGGCAGAGGTACAGCT \\
\hline MDB-1446 & Cb CTB1 3F & TCATGGTCATAGCTGTTTCCTGTGTAACTCCGTCTCCAACCACC \\
\hline MDB-1447 & Cb CTB1 4R & CTGGTCGAGAAACTTGTGCA \\
\hline MDB-1452 & Cb CTB1 5.1F & GAGCGTGCTGTTTCCCTATG \\
\hline
\end{tabular}




\begin{tabular}{|l|l|l|}
\hline MDB-1623 & Cb MEL PKS 1F & ATAGCAGCACCGTACCAACC \\
\hline MDB-1624 & Cb MEL PKS 2R & CACTGGCCGTCGTTTTACAACGTCTGTCTACAGGGAAGGGCATC \\
\hline MDB-1625 & Cb MEL PKS 3F & TCATGGTCATAGCTGTTTCCTGTGTGTCACGGGATAACGAACAA \\
\hline MDB-1626 & Cb MEL PKS 4R & AGTAAGGATTCACGCCGATG \\
\hline MDB-1633 & Cb MEL PKS 5’1F & ACGCAGAGTTTGTCAACACG \\
\hline MDB-1253 & Cb CTB1 gene spec Fp & AGATCGGGATGCCAATCGAC \\
\hline MDB-1254 & Cb CTB1 gene spec Rp & CAATCTCCATGAACTGCGCG \\
\hline MDB-1726 & Cb MEL PKS gene spec Fp & GGTAGCAGCTCCAGTTCCTG \\
\hline MDB-1727 & Cb MEL PKS gene spec Rp & CTCAAAATGAGCGTCGTCAA \\
\hline
\end{tabular}



Chapter 7

General discussion 


\section{Introduction}

The hemibiotrophic fungus Cercospora beticola causes Cercospora leaf spot of sugar beet (1). Due to its incredibly destructive nature and worldwide occurrence, this disease is of great economic importance $(1,2)$. Primary inoculation in the field is initiated when insects, rain or air movements transfer emerging $C$. beticola conidia onto a host plant from overwintering stromata $(1,3)$. Once on a sugar beet leaf, the fungus enters through the stomata and starts colonizing the mesophyll asymptomatically (1). Cercospora leaf spot symptoms will appear after approximately two weeks, depending on environmental conditions (1,4), likely due to a switch from biotrophic to necrotrophic life style of the fungus.

For full virulence, pathogenic microbes must bypass the innate immune system of their desired host plant. This immune system depends on recognition of pathogenic intruders by IPRS (invasion pattern receptors) (5-8). Upon successful recognition, an IP-triggered response (IPTR) is initiated to unveil microbial invasion (6). Unless the invading microbe aims to induce plant defense responses such as hypersensitive responses for its own benefit, it may use effectors to suppress IPTR. Effector proteins have been traditionally described as small, cysteinerich proteins that are deliberately secreted by pathogens to facilitate the infection process. This definition, however, is restrictive in scope and was therefore suggested to be modified to "microbially secreted molecules that contribute to niche colonization" $(9,10)$. The broader definition acknowledges that other microbes, such as endophytes and mutualists, also utilize effectors $(9,10)$. Furthermore, it implies that molecules other than proteins such as secondary metabolites (SMs) (11-13) and small RNAs (14) can fulfill effector functions that may not be limited to the ordinary plant-microbe setting but can also act in microbial competition and nutrition acquisition $(9,10,15)$.

Since our knowledge on the molecular interactions that take place during C. beticola colonization of sugar beet by is limited, this thesis attempts to deepen our knowledge of $C$. beticola infection strategies by investigating its effector biology.

\section{C. beticola proteinaceous effector repertoire}

Effectors are known to be employed by a broad variety of different plant pathogenic fungi to evade detection by the host during colonization. Here, the apoplastic space is one of the locations where interactions between pathogens and host first takes place. Once inside the apoplast, fungal pathogens face defense responses such as chitinases that target fungal cell walls to liberate chitin fragments. These chitin fragments may act as IPs that can be perceived by IPRs, which will lead to an increased release of chitinases into the apoplast with the aim to lyse the cell wall of the invading fungal pathogen (6, 16-19). To shield the fungal cell wall from degradation by plant chitinases, organisms such as the biotrophic fungus Cladosporium fulvum that causes leaf mold on tomato secrete the virulence factor CfAvr4 during infection (20). Due to its peritrophin-A (PAD) domain, a common feature of the carbohydrate-binding module family 14 (CBM14) (21), the CfAvr4 effector is able to bind to chitin in the fungal cell wall and thereby protects fungal hyphae from hydrolysis by plant chitinases (20-24). When investigating 
the existence of CfAvr4 homologs in other Dothideomycetes it was found that several fungi, including C. beticola, harbor a CfAvr4 homolog in their genome $(25,26)$. In vitro carbohydrateaffinity assays with CbAvr4 and other Dothideomycete Avr4 homologs revealed that, like CfAvr4, all Avr4 homologs are able to bind chitin (26). As chitin-binding appears to be a conserved biological trait between CfAvr4 and all Avr4 homologs, it is hypothesized that all Avr4 homologs including CbAvr4 may also share the CfAvr4 function of shielding fungal hyphae from lysis by plant chitinases due to their chitin-binding ability

Effector protein identification through comparative genomics has served as a useful tool to detect another C. beticola effector named CbAve1. CbAve1 is a homolog of VdAve1 (Avirulence on Ve1 tomato), which is secreted by the vascular wilt pathogen Verticillium dahliae. Originally, VdAve1 was identified by comparative population genomics as a linage-specific $V$. dahliae race 1 effector that is recognized by the tomato cell surface-localized immune receptor Ve1 (27). However, in the absence of Ve1, VdAve1 was demonstrated to contribute to fungal virulence (27). Interestingly, VdAve1 homologs are wide-spread and can be found in plants as well as several other plant pathogens. Based on the observation of VdAve1 involvement in virulence, it was also tested whether fungal Ave1 homologs of Fusarium oxysporum (FoAve1), Colletotrichum higginsianum (ChAve1), and CbAve1 are expressed during infection and act as virulence factors (Chapter 3). Gene expression analysis revealed that FoAve1 and CbAve1 are indeed expressed during infection while ChAve1 was not. Moreover, targeted gene replacement of FoAve1, ChAve1, and CbAve1 and subsequent virulence assays on tomato (for $\triangle F o A v e 1$ ), Arabidopsis (for $\triangle C h A v e 1$ ) and sugar beet (for $\triangle C b A v e 1$ ) resulted in a reduced virulence of $\triangle F o A v e 1$ and $\triangle C b A v e 1$ strains compared to their respective wild type strains. Deletion of ChAve1 however, did not have any effect on Co. higginsianum virulence on Arabidopsis. While this outcome indicates that CbAve1 plays a major role in C. beticola virulence, the exact mechanism of how CbAve1 contributes to virulence remains unknown. While current research on VdAve1 function is ongoing, potential results for VdAve1 will have to be experimentally validated for their applicability to CbAve1 as V. dahliae and C. beticola differ in many aspects such as life-style, primary infection site, and infection process.

Due to its hemibiotrophic life style, it was hypothesized that $C$. beticola also secrets effectors that promote the necrotrophic phase of its life cycle. Using a phenotype-based forward genetics approach, a proteinaceous virulence factor named CbNip1 (CbNip for Cercospora beticola necrosis-inducing protein 1) was identified due to its necrosis-inducing activity in sugar beet and Nicotiana benthamiana (Chapter 4). Interestingly, NIP1's ability to induce necrosis within 48 hours was highly regulated by light. While other necrosis-inducing proteins such as ZtNIP1 and ZtNIP2 of the wheat pathogen Zymoseptoria tritici have been shown to need light for full functionality (28), CbNip1 was most active in complete darkness as exposure of CbNip1-infiltrated sugar beet leaf with a 12 hour light-dark cycle led only to chlorosis formation that gradually turned necrotic over time (Chapter 4). Furthermore, CbNip1 appears to contribute to necrotic symptom development, as upregulated CbNip1 expression in planta correlates with necrotic lesion appearance. Unfortunately, the mode-of-action of CbNip1 and its location during infection are currently unknown. While this is the first report 
of a C. beticola effector that depends on darkness for full activity, light-dependent secondary metabolite (SM) effectors have been known for decades to be part of the $C$. beticola armory. These SM effectors are discussed below.

\section{C. beticola secondary metabolites}

Generally fungal SMs are low molecular mass molecules that have no direct contribution to fungal development and reproduction. However, many SMs possess a bioactive nature which is of great value for the producing fungus. The intrinsic modes of action of SMs are remarkably diverse. While some SMs can help with uptake, transport and/or solubilization of metal ions, others are capable of protecting the producing fungus against abiotic and biotic stresses (13). In case of symbiotic fungi, this protection can be extended to also shield the host plant from secondary colonization by other microorganisms. Besides their useful protective function, some SMs of plant pathogenic fungi however are known to play a key role in the fungal infection process and virulence. Classification of SMs that act as toxins can be based on their toxicity range. While host-selective toxins require their host to have a specific target in order to be effective, others exhibit toxicity to a broad spectrum of different organisms and are described as non-host-selective toxins (29). For example, the hemibiotrophic pine needle pathogen Dothistroma septosporum is known to produce the non-host-selective secondary metabolite dothistromin (30). Dothistromin belongs to the anthraquinone family and was shown to contribute to fungal virulence (12). Similar to D. septosporum, C. beticola is also known to produce non-hosts specific toxins, namely beticolins that belong to the xanthraquinone family (31) and the perylenequinone cercosporin (32).

\section{Beticolins}

Beticolins are a group of non-host specific phytotoxins of which 20 members (B0 to B19) have been identified to date to be produced by C. beticola (33-35) and the hoary alyssum (Berteroa incana) pathogen Cercospora berteroae (36). Alternative names for these toxins, such as Gelbe Fraktion (GF) (37), Cercospora beticola toxin (CBT) (36), and cebetins $(38,39)$ arose due to simultaneous research efforts by different groups and limited data concerning their structure during early research. Later, analyses of their chemical structures revealed that beticolins are structurally closely related (Fig. 1). All have a chlorine atom attached to the central aromatic ring, while their octocyclic basic structure is composed of two subunits; a partially hydrogenated anthraquinone and a partially hydrogenated xanthone that are connected through a seven-membered ring (31, 33, 34, 40-42). Structural differences between beticolins are due to different isomeric configurations (ortho-, para-, or epi-ortho-) and by variable residues (31, 43, 44) (Fig. 1). Interestingly, beticolins are able to switch isomery, for example ortho-beticolin $\mathrm{B} 2$ is able to transform into the para-beticolin B1 or epi-ortho-beticolin B6 and vice versa (42) (Fig. 1). Early research on their biological function indicated that beticolins have antibacterial and phytotoxic properties (37). However, necrosis formation in plants upon beticolin application was only induced in the presence of light. Later it was found that due to their ability to form complexes with $\mathrm{Mg}^{2+}$, beticolins inhibit tumoral cell growth in mice (45, 46), interfere with $\mathrm{H}^{+}$-ATPase activity $(40,47-49)$ and are able to incorporate themselves into lipid bilayers to form 


\section{ortho-beticolin}

\section{$\mathrm{B} 2\left(\mathrm{R}=\mathrm{CH}_{3}\right)$}

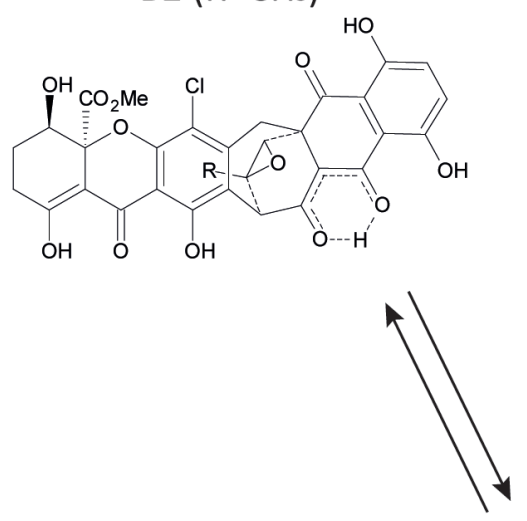

\section{para-beticolin}

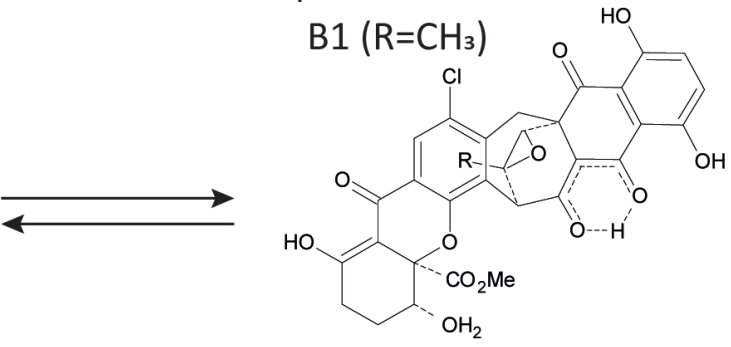

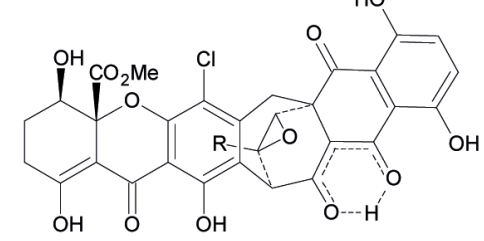

B6 $\left(\mathrm{R}=\mathrm{CH}_{3}\right)$

\section{epi-ortho-beticolin}

Figure 1. Beticolin structures and isoforms. Beticolins are structurally related but can differ by residues (R) and isoforms (ortho-, epi-ortho-, or para-beticolin). Beticolins that carry the same residues are able to transform into each other by switching isomery. For example, the ortho-beticolin B2 that has the the oxygen in ortho position of the clorine atom can transform into the epi-ortho-beticolin B6 or para-beticolin B1 (clorine and oxygen are in para position).

ion channels with poor ion selectivity $(33,34,50)$. The latter property led to the classification of beticolins as ion channel-forming toxins (33). While chemical structures and biological activity have been evaluated throughout the last decades, the biosynthetic pathway of these toxins is unknown. Therefore, it is currently not possible to assess to what extent beticolin production and associated phytotoxic effects contribute to C. beticola virulence.

\section{Cercosporin}

The most prominent and the best-studied example of a non-host selective C. beticola toxin is cercosporin. Cercosporin is produced by most Cercospora species and belongs to the perylenequinone family. Mutant lines that are unable to produce the toxin experience a virulence penalty, indicating that cercosporin is a virulence factor for the species tested (51-53). Toxin production is light-depended as light triggers the induction of the biosynthetic genes responsible for cercosporin formation (11). These genes are organized in a cercosporin toxin biosynthesis 


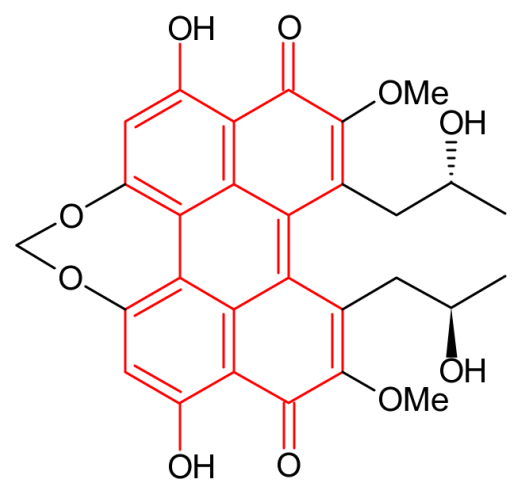

cercosporin<smiles>COc1c2c(c3c4c(OC)c(=O)c5c(O)cc(OC)c(c6c(OC)cc(O)c(c1=O)c64)c53)[C@@H](C(C)=O)[C@H]2C(C)=O</smiles>

elsinochrome A<smiles></smiles>

elsinochrome C<smiles>COc1cc(O)c2c(=O)c(OC)c(C[C@H](C)O)c3c4c(C[C@H](C)O)c(OC)c(=O)c5c(O)cc(OC)c(c5-2)-c1c4-3</smiles>

phleichrome<smiles>COc1c2c(c3c4c(OC)c(=O)c5c(O)cc(OC)c(c6c(OC)cc(O)c(c1=O)c64)c53)[C@H](C(C)=O)[C@H]2C(C)=O</smiles>

elsinochrome B

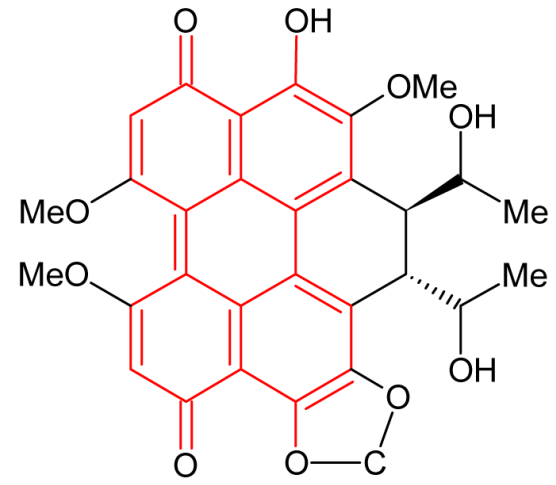

elsinochrome D

Figure 2. Structures of related perylenequinones. All perylenequinones have a common backbone structure (indicated in red) and are therefore structurally related. Structural differences between the molecules are due to differing side chains as can be observed for cercosporin secreted by $C$. beticola, phleichrome by $C$. phlei and elsinochromes A, B, C, and D produced by E. fawcettii. 
cluster (CTB) that was shown to have experienced duplications and multiple horizontal gene transfers during evolution (Chapter 5). Although cercosporin was thought to be a unique feature of fungi that belong to the Cercospora genus, in Chapter 5 we show that the CTB cluster is actually remarkably widely-distributed, including many Colletotrichum species of which Colletotrichum fioriniae has been confirmed to also produce this potent toxin. Due to their indistinctive toxicity, cercosporin and other perylenequinone family members are highly harmful to nearly every living organism (11). In the following section, I will elucidate pathway genes, mode-of-action, and auto-resistance of this destructive-natured but incredibly fascinating perylenequinone toxin and other perylenequinone family members.

\section{Perylenequinone pathway genes}

All perylenequinones are structurally related. Their common feature is a 3,10-dihydroxy-4,9perylenequinone backbone to which every perylenequinone derivate has their own combination of distinct side chains attached (54) (Fig. 2). These side chain variations arise because individual perylenequinone pathways have their own set of decorating enzymes. However, the fact that there are commonalities between perylenequinone structures suggests that also the biosynthetic pathways display significant similarity (Fig. 3). While extensive research on the CTB pathway has shed some light on some putative pathway steps (Fig. 5), it is not possible to determine a full biosynthesis scheme yet due to extreme instability of most pathway intermediates and the potential occurrence of feedback inhibition. Since every CTB gene has an alphanumerical gene name in which the numbers neither represent gene order within the cluster or which step in the pathway an enzyme performs, CTB genes will be discussed in numerical order below.

CTB1 is an iterative NR-PKS and essential for cercosporin production $(52,55,56)$. As PKS genes are the key enzymes for biosynthetic pathways, a CTB1 homolog is present in all perylenequinone clusters (Chapter 5 and 6) (52, 57-59) (Fig. 3). Like all PKS genes, CTB1 harbors multiple functional domains including a starter unit acyltransferase (SAT), a $\beta$-ketoacyl synthase (KS), a malonyl acyltransferase (MAT), a product template domain (PT), a dual-tandem acyl-carrier (ACP $\left.{ }_{2}\right)$ domain, and a thioesterase (TE) domain (55) (Fig. 4). All six catalytic domains conjointly work together to form nor-toralactone, the first intermediate in the cercosporin assembly line (52, 55, 60). Due to its starter unit specificity, CTB1 SAT domain selectively accepts acetyl-CoA as a starter unit (60-62). For initiation of nor-toralactone synthesis, the SAT domain loads the starter unit onto the ACP which in turn is responsible for substrate/product shuffling between the different functional domains (Fig. 4). In a similar fashion as the SAT domain, the MAT domain supplies the ACP with six single malonyl-CoA extender units $(55,56,60)$. Subsequently, the KS domain forms a polyketide chain by attaching one ketide unit at a time through catalyzing consecutive peptide bond formations. Once condensation is complete, the PT domain mediates the characteristic cyclizations and dehydrations of the linear intermediate. Once nor-toralactone formation is completed, the TE domain coordinates the release of the final product from CTB1. Targeted gene replacement of CTB1 as well as of the homolog in the elsinochrome pathway (EfETB1) resulted in complete abolishment of perylenequinone formation (Chapter 5). Loss of CTB1 function and consequently the inability to produce cercosporin was reported to lead to reduced virulence in C. beticola, C. nicotianae and C. kikutchii (51-53). 


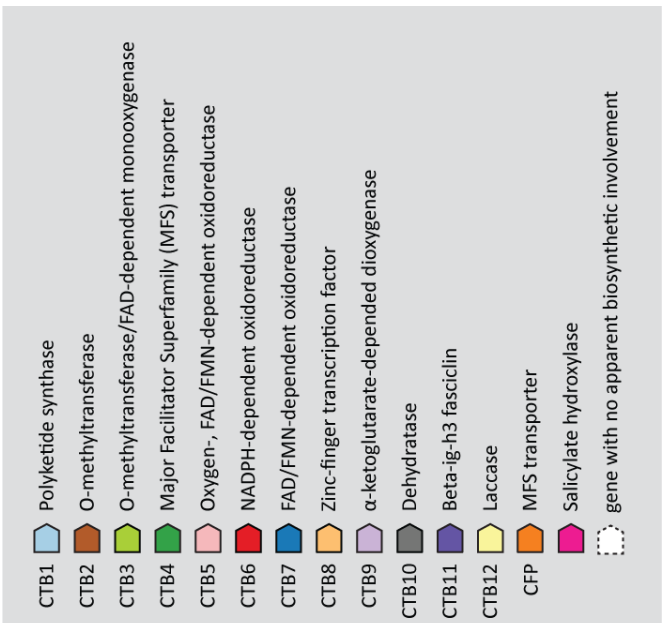

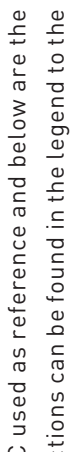

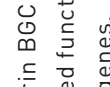

동

जั

㐫

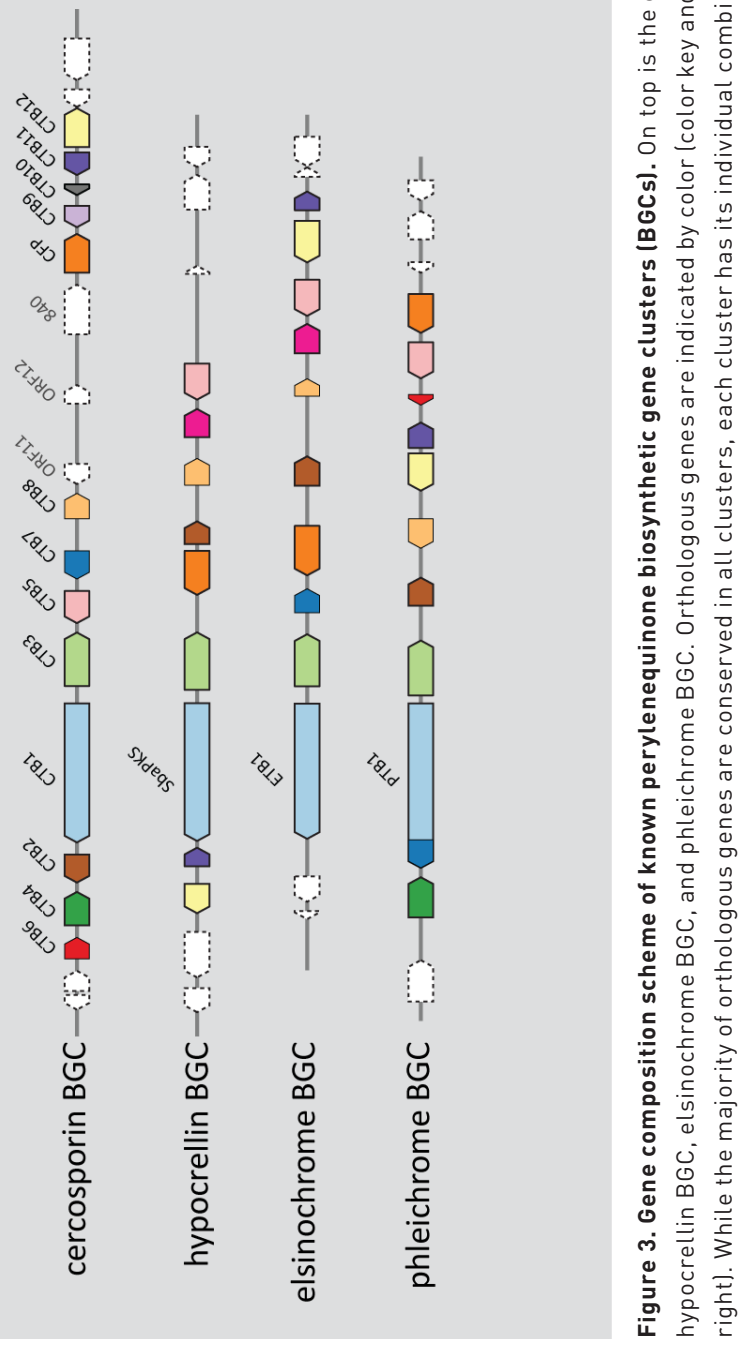




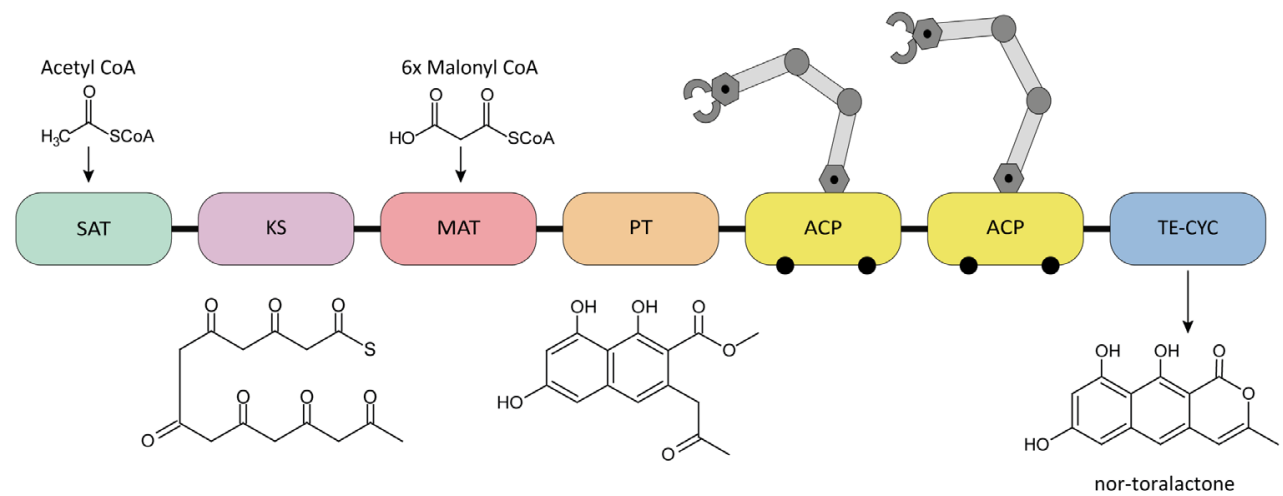

Figure 4. Multidomain structure of the PKS CTB1. Formation of nor-toralactone by CTB1 starts with the starter unit acyltransferase (SAT) specifically accepting acetyl-CoA. Successive condensation in the $\beta$-ketoacyl synthase (KS) domain extends this acetyl-CoA with six malonyl-CoA extender units provided by the malonyl acyltransferase (MAT). Subsequently, the product template domain (PT) will catalyze correct cyclizations and dehydrations of the linear polyketide chain. End product (nor-toralactone) release is mediated by the thioesterase (TE) domain. Throughout the whole process, the ACP domains are responsible for moving substrates and products from one functional domain to the other.

CTB2 is an O-methyltransferase (63) present in the elsinochrome, phleichrome, and hypocrellin clusters (Chapter 6) (Fig. 3). It is hypothesized to mediate intermediate steps of $O$-dimethylation as well as side chain ketone reduction (Fig. 5). Targeted gene deletion of CTB2, resulted in mutant strains that are completely lacking a metabolic profile and showed a severe virulence penalty in planta compared to the cercosporin-producing wild type strain $(60,63,64)$.

CTB3, which is predicted as an O-methyltansferase FAD-depended monooxygenase, is proposed to mediate the second step of cercosporin biosynthesis using the precursor nor-toralactone as substrate (60) (Fig. 5). Interestingly, this enzyme has a dual function as it harbors a putative O-methyltransferase at the $\mathrm{N}$-terminus a and a putative Flavin-dependent monooxygenase domain at the C-terminus $(60,65)$. Individual heterologous expression of each domain revealed that the O-methyltransferase domain is responsible for the conversion of nor-toralactone to toralactone. In turn, toralactone serves as a substrate for CTB3 Flavin-dependent monooxygenase domain which is responsible for the occurrence of an oxidative ring opening. Inoculation assays of CTB3 disruption strains showed that lack of CTB3 impairs fungal virulence (65). Homologs of this enzyme can be found in all known biosynthetic perylenequinone pathways (Chapter 6) (Fig. 3).

CTB4 is a major facilitator superfamily (MFS) transmembrane transporter (64, 66). C. beticola CTB4 disruption strains displayed impairment in cercosporin production by at least $35 \%$ (66). Since cercosporin produced by the CTB4 mutants accumulated in the fungal mycelium and was not secreted into the medium, Choquer et al. (2007) (66) suggested that the lack of CTB4 transporter function impaired cercosporin secretion. However, when stimulated by high light conditions, CTB4 mutants secreted a dark brown compound of unknown nature that quickly diffused into the solid medium (66). Interestingly, a CTB4 homolog is also present in the putative phleichrome biosynthetic 
acetyl-CoA +

$6 \times$ malonyl-CoA<smiles>Cc1cc2cc3cc(O)cc(O)c3c(O)c2c(=O)o1</smiles>

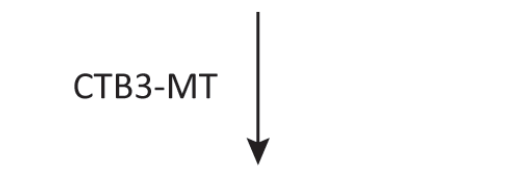<smiles>COc1cc(O)c2c(O)c3c(=O)oc(C)cc3cc2c1</smiles><smiles>COc1cc(O)c2c(c1)C=C(CC(C)=O)C(=O)C2=O</smiles>

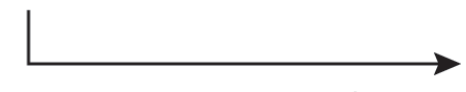

- Dimerization CTB11/12 (??)

- Methylation CTB2

- Reduction CTB6 (??)

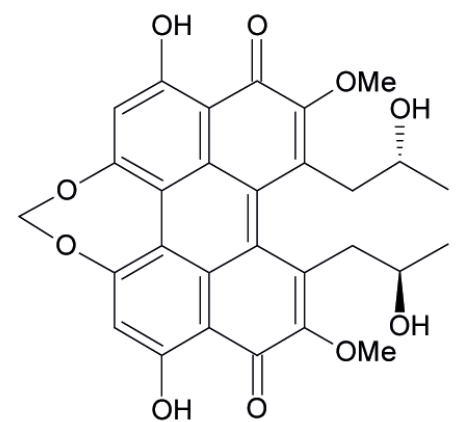

СТВ9/СТВ10<smiles>COc1c(C[C@H](C)O)c2c3c(C[C@H](C)O)c(OC)c4c(O)cc(O)c5c(c(OC)cc(O)c(c1=O)c2c45)c3=O</smiles>

CTB5/СTB7 (??)

Figure 5. Preliminary scheme of the cercosporin biosynthetic pathway consisting of 12 cluster genes. CTB1 forms nor-toralactone which is processed to cercosquinone B by CTB3 methyltransferase (CTB3-MT) and monooxygenase CTB3-MO). Further processing of this cercosporin intermediate might be mediated by CTB2, CTB6, and CTB10 and CTB11 to yield the cercosporin intermediate displayed in a square bracket which has not been directly observed but is rather logically inferred. CTB5 or CTB7 are hypothesized to prime the cercosporin molecule for methylenedioxy bridge formation by СTB9 and CTB10. (Scheme was adopted from chapter 5) 
pathway but missing in the predicted elsinochrome and hypocrellin clusters (Chapter 5) (Fig. 3). As elsinochrome and hypocrellin are secreted by E. fawcettii and S. bambusicola respectively despite the lack of a CTB4 homolog, the question arises whether CTB4 is indeed solely responsible for toxin export in C. nicotianae as suggested by Choquer et al. (2007) (66) or whether other transporter proteins can functionally substitute toxin secretion in the absence of CTB4.

CTB5, a FAD-dependent oxidoreductase, might be involved with single demethylation to an oxidative process linked to CTB9 and CTB10 mediated methylenedioxybridge formation (Chapter 6) (60). However, it is currently unknown whether this step is mediated by CTB5 or CTB7 (Chapter 6) (Fig.5). C. nicotianae mutants lacking CTB5 were reported to secret a dark orange/red monomeric quinone intermediate which was named cercosquinone B. Cercoquinone B is likely an oxidized and therefore stable form of the true naphthalene intermediate which serves as substrate for CTB5. Furthermore, loss of CTB5 functionality resulted in reduced virulence (67). Homologs of this enzyme can also be found in the elsinochrome, phleichrome, and hypocrellin clusters (Chapter 6) (Fig. 3).

CTB6 is a NADPH-dependent oxidoreductase and thought to be the enzyme processing the intermediate formed by CTB2 (Chapter 6) (60) (Fig. 5). Its function is hypothesized to be the stereospecific installment of side chain hydroxyl groups. Inoculations assays revealed that CTB6 disruption mutants of C. nicotianae cause less symptoms compared to the wild type (67). Interestingly, a truncated homolog of this enzyme can be found in the putative phleichrome pathway while no homolog is present in the elsinochrome and hypocrellin pathways (Fig. 3).

CTB7 is a 450 amino acid FAD-binding monooxygenase that harbors two FMN/FAD-binding domains and an amidation site (68). Interestingly, CTB7 is present in a truncated form compared to CTB7 of the CTB clusters of other Cercospora species in the cercosporin biosynthesis pathway of the grey leaf spot pathogen C. zeina. C. zeina with its naturally truncated and therefore nonfunctional CTB7 as well as CTB7 disruption mutants of C. nicotianae are deficient in cercosporin production in vitro $(60,68)$. It was proposed that this enzyme (or CTB5) is involved in the priming of the cercosporin precursor for methylenedioxy bridge installment, likely by removing one aryl methoxy group from the precursor (60) (Fig. 5). Interestingly, this function was already attributed before it was discovered that CTB cluster consists of more than eight genes (Chapter 5). Furthermore, except for the hypocrellin cluster also the elsinochrome and phleichrome cluster harbor this gene, despite that the products of these pathways lack the cercosporin specific methylenedioxy bridge in their structure (Chapter 6) (Fig. 3).

As a $\mathrm{Zn}(\mathrm{II}) \mathrm{Cys}_{6}$ zinc finger transcription factor, CTB8 is not directly involved in the modification of the toxin itself. However, it was shown to mediate CTB cluster gene expression, as CTB8 disruption in C. nicotianae affected transcription of CTB1 through CTB7 (64). The lack of CTB8 regulation also led to severe reduction of CTB1 to CTB7 gene expression levels and consequently to abolishment of cercosporin production in vitro and reduced virulence in planta. While CTB8 is not only tightly incorporated in the CTB cluster in C. beticola, gene homologs are also present in the phleichrome and hypocrellin cluster (Chapter 6) (Fig. 3). However, it seems that the elsinochrome cluster in E. fawcettii harbors a truncated homolog of CTB8 whose functionality has not been studied yet. 
CFP (cercosporin facilitator protein) is another MFS transporter which is tightly incorporated in the CTB cluster of C. beticola (Chapter 5) (69). This transporter is hypothesized to partially provide toxin tolerance to the perylenequinone cercosporin via toxin export (see below: cercosporin auto-resistance) (53). Unlike the other MFS transporter CTB4, CFP homologs can also be found in the predicted phleichrome, elsinochrome, and hypocrellin biosynthetic clusters suggesting a conserved function in perylenequinone auto-resistance (Chapter 6) (Fig. 3).

CTB9 and CTB10 are the only two genes without homologs in any known perylenequinone synthetic clusters (Chapter 6) (Fig. 3). While CTB9 is predicted to be an a-ketoglutarate-depended dioxygenase, CTB10 is suggested to encode a putative dehydratase (Chapter 5). Together they have been shown to be responsible for methylenedioxy bridge formation which is exclusively found in the cercosporin molecule. It is hypothesized that after CTB5- or CTB7-mediated precursor priming, CTB9 induces oxidative cyclization of the peculiar seven-membered ring while CTB10 possibly facilitates this reaction since C. beticola CTB9 and CTB10 deletion mutants were both shown to secret the corresponding precursor of the final cercosporin molecule (Fig. 5).

CTB11 is predicted as a Big-h3 fasciclin that together with the as a laccase annotated CTB12 are hypothesized to be responsible for cercosporin dimerization, a process likely occurring early in the biosynthetic pathway (Chapter 5) (Fig. 5). Contribution to biosynthesis has been displayed when C. beticola mutants lacking either CTB11 or CTB12 were unable to produce cercosporin (Chapter 5). Both enzymes have homologs in elsinochrome, phleichrome and hypocrellin biosynthesis pathways (Chapter 6) (Fig. 3).

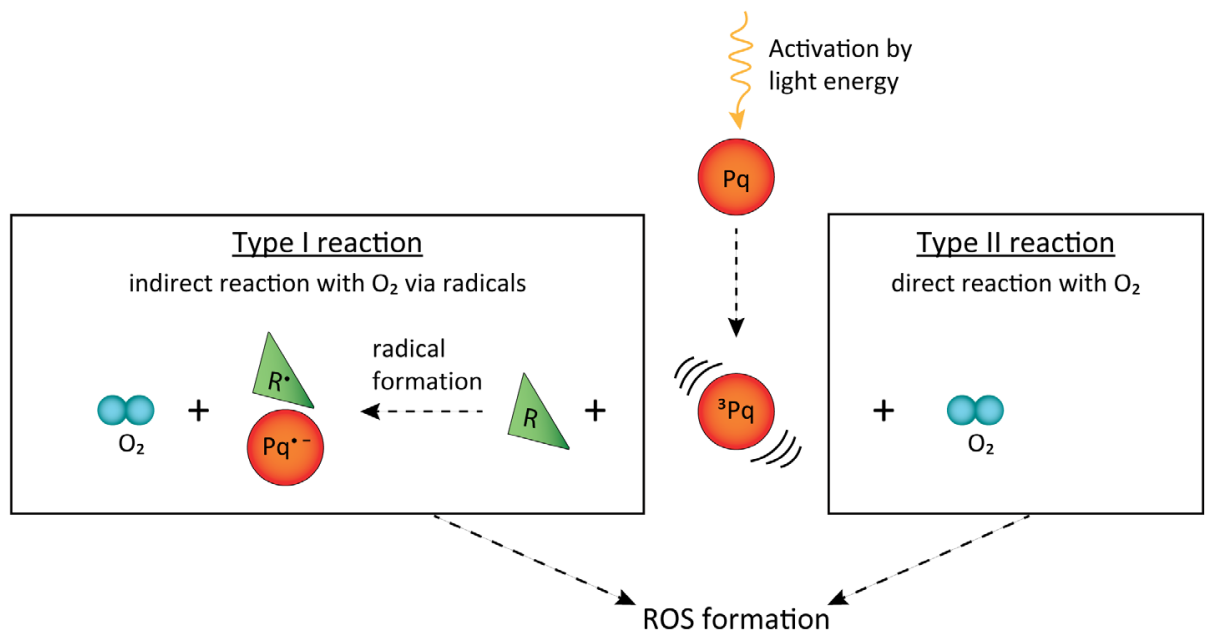

Figure 6. Perylenequinone mode-of-action. Light exposure activates perylenequinones $(\mathrm{Pq})$ to reach an energetically excited triplet state $\left({ }^{3} \mathrm{Pq}\right)$ in which they can react with oxygen $\left(\mathrm{O}_{2}\right)$ to form reactive oxygen species (ROS). This reaction can happen indirectly (type I reaction) where the activated perylenequinone reacts with a reducing substrate $(R)$ first resulting in radical formation $(R \bullet$ and $P q \bullet-)$ which can react with $\mathrm{O}_{2}$ to form $\mathrm{ROS}$. Alternatively, the activated perylenequinone can react directly with $\mathrm{O}_{2}$ (type II reaction) to form ROS. 
Interestingly, the elsinochrome biosynthesis pathway in E. fawcettii and the hypocrellin pathway in S. bambusicola have an additional gene, annotated as a salicylate hydroxylase that has no homologs in the cercosporin or phleichrome pathway (Chapter 5) (Fig. 3). While the exact function of this pathway gene is yet to be determined, other fungal salicylate hydroxylases have been reported to be involved in naphthalene break-down $(70,71)$ and resistance provision in Aspergillus nidulans against the antifungal agent terbinafine (72).

\section{Perylenequinone/cercosporin mode-of-action}

The photosensitizing nature of various perylenequinones such as cercosporin, elsinochromes and fagopyrin has been known for a long time (73-75). The essential common structural feature responsible for photodynamic activity as well as color of the molecule is the 3,10-dihydroxy4,9-perylenequinone chromophore (54). This core structure allows absorption of visible and near-UV light whereby the perylenequinones reach an electronically excited triplet state (76, 77). Once in this activated triplet state, two types of reactions can follow $(76,78,79)$ (Fig. 6).

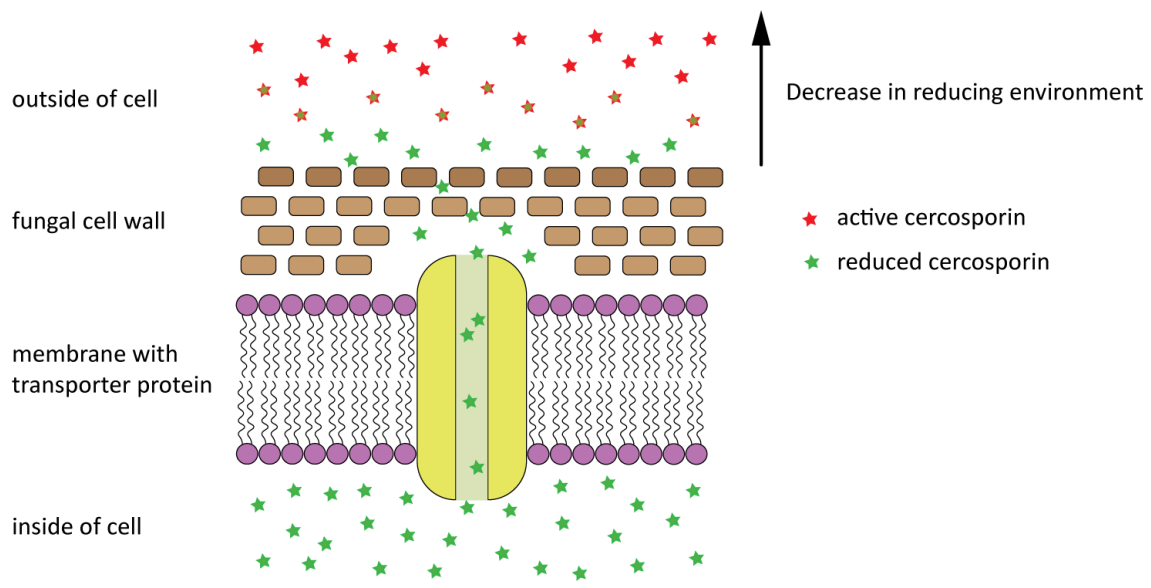

Figure 7. Auto-resistance mechanisms against perylenequinones. Perylenequinone-producing fungi have been shown to protect themselves by exporting the toxic compound outside of the cell via transporter proteins and by detoxification of the produced perylenequinone by creating an environment in which the toxin is getting reduced and therefore less toxic.

The excited perylenequinone can react with oxygen either indirectly (Type I reaction) through a reducing substrate or directly (Type || reaction). Interaction with an electron donor leads to the formation of free radicals or radical ions that upon reaction with oxygen produce reactive oxygen species (ROS) such as $\mathrm{H}_{2} \mathrm{O}_{2}$ and the free radical forms $\mathrm{O}_{2} \bullet-, \mathrm{HO}_{2} \bullet, \mathrm{OH} \bullet$. In a direct interaction between a triplet perylenequinone and oxygen, energy can be transferred from the excited triplet state perylenequinone to oxygen resulting in an excited singlet state of oxygen, also known as "singlet oxygen" $\left({ }^{1} \mathrm{O}_{2}\right)$. Both type I and II reactions yield highly ROS that at high concentrations are harmful to cells as they can cause lipid peroxidation, and protein and DNA damage $(80,81)$. 


\section{Cercosporin auto-resistance}

It is essential for fungi that produce SMs with anti-fungal activity to be able to protect themselves from their own toxin. While genes involved in toxin biosynthesis usually are clustered and form a biosynthesis cluster specific for each secondary metabolite, these clusters quite often also harbor genes that seem to have no direct involvement in toxin production. Nevertheless, these additional genes are conserved within the cluster, suggesting a potential role in toxin tolerance. Keller (2015) (82) highlighted three self-resistance strategies, namely toxin export, detoxification, and duplication of the toxin target, known to date that originate from genes that are incorporated in toxin biosynthetic clusters. While the strategy of duplicating or creating a resistant target deployed by many antifungal toxin producing fungi has not been reported in the context of perylenequinone resistance, toxin export via transporter proteins and detoxification of toxic compounds have been found to be resistance mechanisms utilized by cercosporin-producing fungi (Fig. 7).

\section{Transporter proteins}

Protection by toxin efflux relies on the transportation of toxic substances from the inside of a cell to the outside through membrane transport proteins (Fig. 7). Transporters involved in self-resistance mainly belong to the major facilitator superfamily (MFS) and the ABC superfamily $(83,84)$. Both super families are often associated with multidrug resistance. An example of a transporter utilized by perylenequinone producing fungi is the MFS transporter CFP (53). CFP was first identified in C. kikuchii and shown to be involved in cercosporin auto-resistance. Targeted gene disruption of CFP in C. kikuchii resulted in mutant strains that displayed increased susceptibility to exogenous cercosporin, as their growth on cercosporin-amended media compared to media lacking additional cercosporin was reduced. Additionally, expression of CFP in the cercosporin-sensitive fungus Cochliobolus heterostrophus led to an increased toxin tolerance (85). Furthermore, it was shown that CFP disruption mutants produce much less cercosporin than the WT strain when grown in liquid culture under light conditions, indicating that CFP is also a determining factor for cercosporin production (53), possibly as a self-preservation effect. Studies in C. nicotianae identified three transporters; a CFP homolog and two ABC transporters ATR1 and ATR2 that play a role in cercosporin resistance (86). While gene disruption of CFP or ATR1 resulted in increased sensitivity of the fungus to cercosporin $(66,86)$, overexpression of ATR2 in the cercosporin sensitive fungus Neurospora crassa significantly increased its toxin tolerance (87). However, ATR2 disruption mutants in C. nicotianae were not altered in toxin sensitivity but gene expression analysis revealed that the loss of ATR2 function led to an increased CFP expression. Partial functional compensation was also observed for CFP and ATR1 as constitutive overexpression of ATR1 in CFP disruption mutants partially restored fungal tolerance to cercosporin (86). The potential to provide some level of tolerance against perylenequinones was also observed for ABC and MFS transporter family members identified in non-perylenequinone producing organisms. Native MFS and ABS transporters in Botrytis cinerea and the yeast Saccharomyces cerevisiae, respectively, have been reported to confer cercosporin resistance when overexpressed $(88,89)$. Interestingly, it was possible to transfer this transporter-based resistance mechanism to plants. For example, stable transformation of fungal-derived CFP in tobacco resulted in CFP+ transgenic plants with increased tolerance to cercosporin since application of external cercosporin on the leaves as well as infection with C. nicotianae resulted in smaller necrotic lesion sizes (90). With the discovery 
that the CTB cluster consists of more genes than previously identified eight CTB genes, it was possible to demonstrate that the MFS transporter homolog in C. beticola CbCFP, also involved in auto-resistance, actually lies within the CTB cluster (Chapter 5) (69). In fact, it is tightly incorporated in the cluster, flanked from both sides by genes necessary for cercosporin production (Fig. 3). Its location within the cluster illustrates the close connection between toxin biosynthesis and autoresistance and ensures conservation of toxin tolerance together with the mycotoxin production pathway as loss of self-resistance would be devastating for the fungus.

\section{Detoxification by alteration of the toxin structure}

Besides toxin export, the active modification of a toxin into a less toxic derivate is another selfprotection strategy used by many fungi (82). Instead of depending on only one resistance mechanism, the auto-resistance repertoire of Cercospora spp. also includes the ability to defend against cercosporin by reductive detoxification of the cercosporin molecules (91-94). Although reduced cercosporin is rather labile and readily re-oxidizes upon removal of reducing agents or through air exposure $(94,95)$, analysis of stable methylated and acetylated reduced cercosporin derivatives revealed that they absorb less light and generate significantly less singlet oxygen $\left({ }^{1} \mathrm{O}_{2}\right)$ compared to wild-type cercosporin (94). Consequently, their potential to cause lipid peroxidation in vitro was highly reduced and cercosporin-susceptible fungi grew significantly bigger on media amended with reduced cercosporin compared to wild-type cercosporin containing media (94). Furthermore, it was found that ${ }^{1} \mathrm{O}_{2}$ production by reduced cercosporin is highly influenced by its chemical environment with a distinct low in an aqueous setting as it can be found inside of cells (91). Through fluorescence microscopy with specific band-width filters, it was possible to discriminate reduced cercosporin from wild-type cercosporin (92). Interestingly, when grown in the presence of wild-type cercosporin, hyphae of Cercospora species as well as a cercosporin-resistant Alternaria alternata strain were not emitting red wild-type cercosporin-specific fluorescence but green fluorescence associated with reduced form of cercosporin. The reduced cercosporin was later found to be localized in the cytoplasm of the fungal cells (91). Non-viable Cercospora spp. and cercosporin-sensitive N. crassa and Aspergillus flavus strains on the other hand were unable or highly limited in their abilities to reduce cercosporin and therefore emitted red wild-type cercosporin specific fluorescence (92). Further investigations revealed that the cell surface of cercosporinresistant strains is surrounded by a reducing environment (94). Thereby resistant fungi are able to reduce nearby cercosporin into its less reactive form and keep it in this state as long as it is in close proximity to the fungal hyphae (Fig. 7). The importance of reduction as a defense mechanism for oxidative stress tolerance was supported by the discovery of the transmembrane reductase Cpd1 (cercosporin and photosensitizer detoxification) in yeast (89). Mutants over-expressing Cpd1 showed an increased resistance to cercosporin and other synthetic photosensitizers whereas disruption of the gene lead to increased susceptibility. Furthermore, Cpd1 is able to increase cercosporin tolerance of plants as leaves of transgenic tobacco plants expressing Cdp1 were less affected by infiltration of pure cercosporin (96). Although the exact functional mechanism of Cpd1 is still unknown, Cpd1 shows significant similarities FAD-dependent pyridine nucleotide reductases suggesting a possible role in reduction of the cercosporin molecule. Although it is reported for other fungi that genes linked to active toxin detoxification can be found incorporated in the biosynthesis cluster, no CTB genes have been affiliated with auto-resistance by cercosporin reduction yet. 


\section{Other resistance mechanisms}

While toxin efflux and detoxification have been shown to be resistance strategies of perylenequinone-producing fungi, studies on self-resistance also led to the identification of other resistance mechanisms and genes that play a role in toxin tolerance but for which the underlying mechanisms are not yet fully understood. Interestingly, a gene involved in vitamin $\mathrm{B}_{6}$ (pyridoxine) biosynthesis is also among the additionally identified resistance-providing genes (97). SOR1 (Singlet Oxygen Resistance 1), later renamed to PDX1 based on its pyridoxine auxotrophy phenotype, was identified due to its ability to restore cercosporin resistance in an UVderived toxin-sensitive mutant $(98,99)$. Targeted gene replacement of PDX1 resulted in increased susceptibility to cercosporin and other photosensitizers. Since further research revealed that PDX1 is required for vitamin $\mathrm{B}_{6}$ formation, the role of pyridoxine and its derivatives in protection against photosensitizers was investigated further $(97,100)$. Pyridoxine, pyridoxal, pyridoxal 5-phosphate, and pyridoxamine were all found to be potent ${ }^{1} \mathrm{O}_{2}$ quencher and therefore are likely contributing to oxidative stress resistance caused by cercosporin and other photosensitizers.

Another example is the transcription factor Crg1 (cercosporin resistance gene) identified in C. nicotianae $(101,102)$. Crg1 was found to be partially involved in cercosporin auto-resistance as C. nicotianae Crg1 disruption mutants display significantly impaired growth on cercosporinamended media. However, growth of Crg1 disruption mutants was not significantly altered by the presence of other ${ }^{1} \mathrm{O}_{2}$ generating photosensitizers (eosin $Y$ and toluidine blue). This finding indicates that CRG1 seems to regulate specific genes involved in cercosporin tolerance rather than genes important for ${ }^{1} \mathrm{O}_{2}$ resistance in general.

\section{Conclusion}

While knowledge on the effector biology of the hemibiotrophic sugar beet pathogen C. beticola is limited, research aiming to identify and characterize new effectors is viable to understand the underlying molecular strategies that this fungus employs to establish disease. The currently known effector repertoire of $C$. beticola indicates that this fungus utilizes proteinaceous as well as SM effectors during infection. Furthermore, it appears that during its necrotrophic phase, $C$. beticola is equipped with specific necrosis-inducing effectors for light (cercosporin) and dark periods (CbNip1). The broad toxicity of the perylenequinone cercosporin makes it dangerous not only to plants but potentially also to other living organisms. With the discovery that the ability to synthesize cercosporin is not limited to the Cercospora genus a new level of complexity on perylenequinones as potential health threats emerged since perylenequinoneproducing pathogens may secrete these toxins into plant products that are directly consumed by mammals. The increased use of next-generation sequencing combined with bioinformatics and molecular-biological approaches can help to uncover latent perylenequinone producers and will shed light on yet unexplored areas of perylenequinone research. To date, our knowledge on underlying auto-resistance mechanisms is still limited. However new insights in this field will help to increase our understanding of how toxin tolerance is achieved by fungi and can potentially lead to new developments for modern agricultural farming. 


\section{References}

1. Weiland J \& Koch G (2004) Sugarbeet leaf spot disease (Cercospora beticola sacc.). Molecular plant pathology 5(3):157-166

2. Secor GA, Rivera VV, Khan MFR, \& Gudmestad NC (2010) Monitoring fungicide sensitivity of Cercospora beticola of sugar beet for disease management decisions. Plant Disease 94(11):1272-1282.

3. Khan M \& Khan J (2010) Survival, spore trapping, dispersal, and primary infection site for Cercospora beticola in sugarbeet. Cercospora leaf spot of sugar beet and related species, ed R.T. Lartey JJW, L. Panella, P.W. Crous, and C.E. Windels (APS Press, St. Paul), Vol 96, pp 67-75.

4. Weltmeier F, et al. (2011) Transcript profiles in sugar beet genotypes uncover timing and strength of defense reactions to Cercospora beticola infection. Molecular Plant-Microbe Interactions 24(7):758-772.

5. Jones JDG, Vance RE, \& Dangl JL (2016) Intracellular innate immune surveillance devices in plants and animals. Science 354(6316).

6. Cook DE, Mesarich CH, \& Thomma BP (2015) Understanding plant immunity as a surveillance system to detect invasion. Annual review of phytopathology 53:541-563.

7. Zipfel C (2008) Pattern-recognition receptors in plant innate immunity. Current Opinion in Immunology 20(1):10-16.

8. Tena G, Boudsocq M, \& Sheen J (2011) Protein kinase signaling networks in plant innate immunity. Current Opinion in Plant Biology 14(5):519-529.

9. Rovenich H, Boshoven JC, \& Thomma BPHJ (2014) Filamentous pathogen effector functions: Of pathogens, hosts and microbiomes. Current Opinion in Plant Biology 20:96-103.

10. Snelders NC, Kettles GJ, Rudd JJ, \& Thomma BPHJ (2018) Plant pathogen effector proteins as manipulators of host microbiomes? Molecular Plant Pathology 19(2):257-259.

11. Daub ME \& Ehrenshaft M (2000) The photoactivated Cercospora toxin cercosporin: Contributions to plant disease and fundamental biology. Annual review of phytopathology 38(1):461-490.

12. Kabir M, Ganley R, \& Bradshaw R (2015) Dothistromin toxin is a virulence factor in dothistroma needle blight of pines. Plant pathology 64(1):225-234.

13. Demain AL \& Fang A (2000) The natural functions of secondary metabolites. History of modern biotechnology $i$, (Springer), pp 1-39.

14. Wang M, et al. (2016) Bidirectional cross-kingdom RNAi and fungal uptake of external RNAs confer plant protection. Nature Plants 2:16151.

15. Fatima U \& Senthil-Kumar M (2015) Plant and pathogen nutrient acquisition strategies. Frontiers in Plant Science 6(750).

16. Sánchez-Vallet A, Mesters JR, \& Thomma BPHJ (2015) The battle for chitin recognition in plant-microbe interactions. FEMS Microbiology Reviews 39(2):171-183.

17. Fesel PH \& Zuccaro A (2016) B-glucan: Crucial component of the fungal cell wall and elusive mamp in plants. Fungal Genetics and Biology 90:53-60.

18. Kombrink A, Sánchez-Vallet A, \& Thomma BPHJ (2011) The role of chitin detection in plant-pathogen interactions. Microbes and Infection 13(14):1168-1176.

19. Felix G, Regenass M, \& Boller T (1993) Specific perception of subnanomolar concentrations of chitin fragments by tomato cells: Induction of extracellular alkalinization, changes in protein phosphorylation, and establishment of a refractory state. The Plant Journal 4(2):307-316.

20. van Esse HP, Bolton MD, Stergiopoulos I, de Wit PJGM, \& Thomma BPHJ (2007) The chitin-binding Cladosporium fulvum effector protein Avr4 is a virulence factor. Molecular Plant-Microbe Interactions 20(9):1092-1101.

21. Chang T-C \& Stergiopoulos I (2015) Inter- and intra-domain horizontal gene transfer, gain-loss asymmetry and positive selection mark the evolutionary history of the CBM14 family. FEBS Journal 282(10):2014-2028.

22. van den Burg HA, Harrison SJ, Joosten MHAJ, Vervoort J, \& de Wit PJGM (2006) Cladosporium fulvum Avr4 protects fungal cell walls against hydrolysis by plant chitinases accumulating during infection. Molecular PlantMicrobe Interactions 19(12):1420-1430.

23. van den Burg HA, et al. (2004) Binding of the Avr4 elicitor of Cladosporium fulvum to chitotriose units is facilitated by positive allosteric protein-protein interactions: The chitin-binding site of Avr4 represents a novel binding site on the folding scaffold shared between the invertebrate and the plant chitin-binding domain. Journal of Biological Chemistry 279(16):16786-16796.

24. van den Burg HA, et al. (2003) Natural disulfide bond-disrupted mutants of Avr4 of the tomato pathogen Cladosporium fulvum are sensitive to proteolysis, circumvent Cf-4-mediated resistance, but retain their chitin binding ability. Journal of Biological Chemistry 278(30):27340-27346

25. Stergiopoulos I, et al. (2010) Tomato cf resistance proteins mediate recognition of cognate homologous effectors from fungi pathogenic on dicots and monocots. Proceedings of the National Academy of Sciences 107(16):7610-7615.

26. Mesarich $\mathrm{CH}$, et al. (2016) A conserved proline residue in Dothideomycete Avr4 effector proteins is required to trigger a Cf-4-dependent hypersensitive response. Molecular Plant Pathology 17(1):84-95.

27. de Jonge R, et al. (2012) Tomato immune receptor Ve1 recognizes effector of multiple fungal pathogens uncovered by genome and RNA sequencing. Proceedings of the National Academy of Sciences 109(13):5110-5115.

28. Ben M'Barek S, et al. (2015) FPLC and liquid-chromatography mass spectrometry identify candidate necrosis-inducing proteins from culture filtrates of the fungal wheat pathogen Zymoseptoria tritici. Fungal Genet Biol 79:54-62. 
29. Wolpert TJ, Dunkle LD, \& Ciuffetti LM (2002) Host-selective toxins and avirulence determinants: What's in a name? Annual review of phytopathology 40(1):251-285.

30. Bradshaw R (2004) Dothistroma (red-band) needle blight of pines and the dothistromin toxin: A review. Forest Pathology 34(3):163-185

31. Milat M-L, Prange T, Wiedemann-Merdinoglu S, \& Blein J-P (2010) Beticolins: Chemistry and biological activities. in Cercospora leaf spot of sugar beet and related species, eds Robert T, Lartey JJW, Weiland JJ, Panella L, Crous PW, \& Windels CE (APS press, St Paul Minnesota, USA ), pp 119-128.

32. Lousberg RC, et al. (1971) The structure of cercosporin, a naturally occurring quinone. Journal of the Chemical Society D: Chemical Communications (22):1463-1464.

33. Goudet C, Milat M-L, Sentenac H, \& Thibaud J-B (2000) Beticolins, nonpeptidic, polycyclic molecules produced by the phytopathogenic fungus Cercospora beticola, as a new family of ion channel-forming toxins. Molecular Plant-Microbe Interactions 13(2):203-209.

34. Goudet C, et al. (1998) Magnesium ions promote assembly of channel-like structures from beticolin 0, a nonpeptide fungal toxin purified from Cercospora beticola. The Plant Journal 14(3):359-364.

35. Milat M-L \& Blein J-P (1995) Cercospora beticola toxins III. Purification, thin-layer and high performance liquid chromatographic analyses. Journal of Chromatography A 699(1):277-283.

36. Assante G, Locci R, Camarda L, Merlini L, \& Nasini G (1977) Screening of the genus Cercospora for secondary metabolites. Phytochemistry 16(2):243-247.

37. Schlösser E (1962) Über eine biologisch aktive Substanz aus Cercospora beticola. Journal of Phytopathology 44(3):295-312.

38. Jalal MA, Hossain MB, Robeson DJ, \& Van der Helm D (1992) Cercospora beticola phytotoxins: Cebetins that are photoactive, magnesium ion-binding, chlorinated anthraquinone-xanthone conjugates. Journal of the American Chemical Society 114(15):5967-5971.

39. Robeson DJ \& Jalal MAF (1993) A Cercospora isolate from soybean roots produces cebetin b and cercosporin. Phytochemistry 33(6):1546-1548

40. Simon-Plas F, Gomes E, Milat ML, Pugin A, \& Blein JP (1996) Cercospora beticola toxins (X. Inhibition of plasma membrane $\mathrm{H}^{+}$-ATPase by beticolin-1). Plant Physiology 111(3):773-779.

41. Ducrot PH, et al. (1996) Cercospora beticola toxins. Part XI1: Isolation and structure of beticolin 0. Tetrahedron Letters 37(18):3121-3124.

42. Ducrot PH, Lallemand JY, Milat ML, \& Blein JP (1994) The yellow toxins produced by Cercospora beticola. Part VIII : Chemical equilibrium between beticolins; structures of minor compounds: Beticolin 6 and beticolin 8 . Tetrahedron Letters 35(47):8797-8800.

43. Milat M, et al. (1992) Structures of the beticolins, the yellow toxins produced by Cercospora beticola. Journal of the American Chemical Society 114(4):1478-1479.

44. Prangé T, Neuman A, Milat M-L, \& Blein J-P (1997) Cercospora beticola toxins. Part 16. X-ray diffraction analyses on microcrystals of three $p$-beticolins. Journal of the Chemical Society, Perkin Transactions 2 (9):1819-1826.

45. Ding G, et al. (1996) Inhibition of cellular growth and steroid 11ß-hydroxylation in ras-transformed adrenocortical cells by the fungal toxins beticolins. Cell Biology International 20(8):523-530.

46. Ding GQ, et al. (2001) Effects of 12 beticolins, Cercospora beticola toxins, on proliferation of ras-transformed adrenocortical cell. Acta Pharmacol Sin 22(9):769-776.

47. Gomès E, et al. (1996) Cercospora beticola toxins. Part XVII. The role of the beticolin $/ \mathrm{Mg}_{2}{ }^{+}$complexes in their biological activity study of plasma membrane $\mathrm{H}^{+}$-ATPase, vacuolar $\mathrm{H}^{+}$-PPase, alkaline and acid phosphatases. Biochimica et Biophysica Acta (BBA) - Biomembranes 1285(1):38-46.

48. Gomés E, et al. (1996) Activation of the plant plasma membrane $\mathrm{H}^{+}$-ATPase. Is there a direct interaction between lysophosphatidylcholine and the C-terminal part of the enzyme? FEBS Letters 398(1):48-52.

49. Gomés E, et al. (1996) Cercospora beticola toxins. IX. Relationship between structure of beticolins, inhibition of plasma membrane $\mathrm{H}^{+}$-ATPase and partition in lipid membranes. Physiologia Plantarum 98(1):133-139.

50. Goudet C, Benitah J-P, Milat M-L, Sentenac H, \& Thibaud J-B (1999) Cluster organization and pore structure of ion channels formed by beticolin 3, a nonpeptidic fungal toxin. Biophysical Journal 77(6):3052-3059.

51. Weiland JJ, Chung K-R, \& Suttle JC (2010) The role of cercosporin in the virulence of Cercospora spp. To plant hosts. Cercospora leaf spot of sugar beet and related species, eds Lartey RT, Weiland JJ, Panella L, Crous PW, \& Windels CE (APS Press, St. Paul).

52. Choquer $M$, et al. (2005) The CTB1 gene encoding a fungal polyketide synthase is required for cercosporin biosynthesis and fungal virulence of Cercospora nicotianae. Molecular plant-microbe interactions 18(5):468-476.

53. Callahan TM, Rose MS, Meade MJ, Ehrenshaft M, \& Upchurch RG (1999) CFP, the putative cercosporin transporter of Cercospora kikuchii, is required for wild type cercosporin production, resistance, and virulence on soybean. Molecular plant-microbe interactions 12(10):901-910.

54. Hudson J, Imperial V, Haugland R, \& Diwu Z (1997) Antiviral activities of photoactive perylenequinones. Photochemistry and photobiology 65(2):352-354.

55. Newman AG, Vagstad AL, Belecki K, Scheerer JR, \& Townsend CA (2012) Analysis of the cercosporin polyketide synthase CTB1 reveals a new fungal thioesterase function. Chemical Communications 48(96):11772-11774.

56. Crawford JM \& Townsend CA (2010) New insights into the formation of fungal aromatic polyketides. Nature Reviews Microbiology 8(12):879-889. 
57. Gallo A, Ferrara M, \& Perrone G (2013) Phylogenetic study of polyketide synthases and nonribosomal peptide synthetases involved in the biosynthesis of mycotoxins. Toxins 5(4):717-742.

58. Brakhage AA (2013) Regulation of fungal secondary metabolism. Nature Reviews Microbiology 11(1):21-32.

59. Keller NP, Turner G, \& Bennett JW (2005) Fungal secondary metabolism-from biochemistry to genomics. Nature Reviews Microbiology 3(12):937-947.

60. Newman AG \& Townsend CA (2016) Molecular characterization of the cercosporin biosynthetic pathway in the fungal plant pathogen Cercospora nicotianae. Journal of the American Chemical Society.

61. Crawford JM, Vagstad AL, Ehrlich KC, \& Townsend CA (2008) Starter unit specificity directs genome mining of polyketide synthase pathways in fungi. Bioorganic Chemistry 36(1):16-22

62. Crawford JM, Vagstad AL, Whitworth KP, Ehrlich KC, \& Townsend CA (2008) Synthetic strategy of nonreducing iterative polyketide synthases and the origin of the classical "starter-unit effect". ChemBioChem 9(7):1019-1023.

63. Staerkel C, etal. (2013) Cbctb2, an o-methyltransferase is essential for biosynthesis of the phytotoxin cercosporin and infection of sugar beet by Cercospora beticola. BMC plant biology 13(1):50.

64. Chen H, Lee MH, Daub ME, \& Chung KR (2007) Molecular analysis of the cercosporin biosynthetic gene cluster in Cercospora nicotianae. Molecular microbiology 64(3):755-770.

65. Dekkers KL, et al. (2007) The Cercospora nicotianae gene encoding dual O-methyltransferase and FAD-dependent monooxygenase domains mediates cercosporin toxin biosynthesis. Fungal Genetics and Biology 44(5):444-454.

66. Choquer M, Lee M-H, Bau H-J, \& Chung K-R (2007) Deletion of a MFS transporter-like gene in Cercospora nicotianae reduces cercosporin toxin accumulation and fungal virulence. FEBS Letters 581(3):489-494

67. Chen $H-Q$, Lee $M-H$, \& Chung K-R (2007) Functional characterization of three genes encoding putative oxidoreductases required for cercosporin toxin biosynthesis in the fungus Cercospora nicotianae. Microbiology 153(8):2781-2790

68. Swart V, et al. (2017) Complementation of CTB7 in the maize pathogen Cercospora zeina overcomes the lack of in vitro cercosporin production. Molecular Plant-Microbe Interactions 30(9):710-724.

69. de Jonge R, et al. (2018) Gene cluster conservation provides insight into cercosporin biosynthesis and extends production to the genus Colletotrichum. Proceedings of the National Academy of Sciences, doi:10.1073/ pnas.1712798115.

70. Rabe F, Ajami-Rashidi Z, Doehlemann G, Kahmann R, \& Djamei A (2013) Degradation of the plant defence hormone salicylic acid by the biotrophic fungus Ustilago maydis. Mol Microbiol 89(1):179-188.

71. Dodge AG \& Wackett LP (2005) Metabolism of bismuth subsalicylate and intracellular accumulation of bismuth by Fusarium sp. strain BI. Applied and environmental microbiology 71(2):876-882

72. Graminha MA, Rocha EM, Prade RA, \& Martinez-Rossi NM (2004) Terbinafine resistance mediated by salicylate 1-monooxygenase in Aspergillus nidulans. Antimicrobial agents and chemotherapy 48(9):3530-3535

73. Yamazaki S, Okubo A, Akiyama Y, \& Fuwa K (1975) Cercosporin, a novel photodynamic pigment isolated from Cercospora kikuchii. Agricultural and Biological Chemistry 39(1):287-288.

74. Weiss U, Flon H, \& Burger WC (1957) The photodynamic pigment of some species of Elsinoe and Sphaceloma. Archives of biochemistry and biophysics 69:311-319.

75. Brockmann H, Weber E, \& Sander E (1950) Fagopyrin, ein photodynamischer Farbstoff aus Buchweizen (fagopyrum esculentum). Naturwissenschaften 37(2):43-43.

76. Foote CS (1976) Photosensitized oxidation and singlet oxygen. Free radicals in biology 2:85-133

77. Foote CS (1968) Mechanisms of photosensitized oxidation. Science 162(3857):963-970.

78. Guedes RC \& Eriksson LA (2007) Photophysics, photochemistry, and reactivity: Molecular aspects of perylenequinone reactions. Photochemical \& Photobiological Sciences 6(10):1089-1096.

79. DeRosa MC \& Crutchley RJ (2002) Photosensitized singlet oxygen and its applications. Coordination Chemistry Reviews 233:351-371

80. Birben E, Sahiner UM, Sackesen C, Erzurum S, \& Kalayci O (2012) Oxidative stress and antioxidant defense. World Allergy Organization Journal 5(1):9.

81. Blokhina O, Virolainen E, \& Fagerstedt KV (2003) Antioxidants, oxidative damage and oxygen deprivation stress: A review. Annals of botany 91(2):179-194.

82. Keller NP (2015) Translating biosynthetic gene clusters into fungal armor and weaponry. Nature chemical biology 11(9):671-677.

83. Costa C, Dias PJ, Sá-Correia I, \& Teixeira MC (2014) MFS multidrug transporters in pathogenic fungi: Do they have real clinical impact? Frontiers in physiology 5.

84. Cannon RD, et al. (2009) Efflux-mediated antifungal drug resistance. Clinical microbiology reviews 22(2):291-321.

85. Upchurch RG, Rose MS, Eweida M, \& Callahan TM (2002) Transgenic assessment of CFP-mediated cercosporin export and resistance in a cercosporin-sensitive fungus. Current genetics 41(1):25-30.

86. Amnuaykanjanasin A \& Daub ME (2009) The ABC transporter ATR1 is necessary for efflux of the toxin cercosporin in the fungus Cercospora nicotianae. Fungal genetics and biology 46(2):146-158.

87. Beseli A, Amnuaykanjanasin A, Herrero S, Thomas E, \& Daub ME (2015) Membrane transporters in self resistance of Cercospora nicotianae to the photoactivated toxin cercosporin. Current genetics 61(4):601-620.

88. Hayashi K, Schoonbeek H-j, \& De Waard MA (2002) Bcmfs1, a novel major facilitator superfamily transporter from Botrytis cinerea, provides tolerance towards the natural toxic compounds camptothecin and cercosporin and towards fungicides. Applied and environmental microbiology 68(10):4996-5004. 
89. Ververidis $\mathrm{P}$, et al. (2001) A novel putative reductase (Cpd1p) and the multidrug exporter Snq2p are involved in resistance to cercosporin and other singlet oxygen-generating photosensitizers in saccharomyces cerevisiae. Current genetics 39(3).

90. Upchurch RG, Rose MS, Eweida M, \& Zuo W (2005) Expression of the cercosporin transporter, CFP, in tobacco reduces frog-eye lesion size. Biotechnology letters 27(20):1543-1550.

91. Daub ME, Li M, Bilski P, \& Chignell CF (2000) Symposium-in-print dihydrocercosporin singlet oxygen production and subcellular localization: A possible defense against cercosporin phototoxicity in Cercospora. Photochemistry and photobiology 71(2):135-140.

92. Daub ME, Leisman GB, Clark RA, \& Bowden EF (1992) Reductive detoxification as a mechanism of fungal resistance to singlet oxygen-generating photosensitizers. Proceedings of the National Academy of Sciences $89(20): 9588-9592$.

93. Cooperman Sollod C, Jenns AE, \& Daub ME (1992) Cell surface redox potential as a mechanism of defense against photosensitizers in fungi. Applied and environmental microbiology 58(2):444-449.

94. Leisman GB \& Daub ME (1992) Singlet oxygen yields, optical properties, and phototoxicity of reduced derivatives of the photosensitizer cercosporin. Photochemistry and photobiology 55(3):373-379.

95. Daub ME, Herrero S, \& Chung K-R (2005) Photoactivated perylenequinone toxins in fungal pathogenesis of plants. FEMS Microbiology Letters 252(2):197-206

96. Panagiotis $M$, et al. (2007) Expression of the yeast cpd1 gene in tobacco confers resistance to the fungal toxin cercosporin. Biomolecular engineering 24(2):245-251.

97. Ehrenshaft M, Bilski P, Li MY, Chignell CF, \& Daub ME (1999) A highly conserved sequence is a novel gene involved in de novo vitamin B6 biosynthesis. Proceedings of the National Academy of Sciences 96(16):9374-9378.

98. Ehrenshaft M, Chung K-R, Jenns AE, \& Daub ME (1999) Functional characterization of SOR1, a gene required for resistance to photosensitizing toxins in the fungus Cercospora nicotianae. Current genetics 34(6):478-485.

99. Ehrenshaft M, Jenns A, Chung K, \& Daub M (1998) SOR1, a gene required for photosensitizer and singlet oxygen resistance in Cercospora fungi, is highly conserved in divergent organisms. Molecular cell 1(4):603-609.

100. Bilski P, Li M, Ehrenshaft M, Daub M, \& Chignell C (2000) Symposium-in-print vitamin B6 (pyridoxine) and its derivatives are efficient singlet oxygen quenchers and potential fungal antioxidants. Photochemistry and Photobiology 71(2):129-134

101. Chung K-R, Daub ME, Kuchler K, \& Schüller C (2003) The CRG1 gene required for resistance to the singlet oxygen-generating cercosporin toxin in Cercospora nicotianae encodes a putative fungal transcription factor. Biochemical and biophysical research communications 302(2):302-310.

102. Chung KR, Jenns AE, Ehrenshaft M, \& Daub ME (1999) A novel gene required for cercosporin toxin resistance in the fungus Cercospora nicotianae. Mol Gen Genet 262(2):382-389. 



\section{Summary}

Plants possess an innate immune system that enables them to detect microbial invasion and respond accordingly to prevent disease. In turn, microbes have evolved secreted molecules that are collectively termed effectors to overcome recognition by the plant and facilitate host colonization. In Chapter 1, past and current conceptual models depicting plant-microbe interplays during infection are addressed. Additionally, the primary subject of this thesis, the sugar beet pathogen Cercospora beticola, is introduced.

Depending on their life style, plant pathogenic fungi employ versatile virulence strategies during colonization. Chapter 2 provides a broad overview of virulence mechanisms that are utilized by various pathogenic microbes. Furthermore, it highlights different kinds of effectors such as proteins, small RNAs, and secondary metabolites, and their function in context of fungal virulence.

An example of a proteinaceous effector in C. beticola is CbAve1, a homolog of the Verticillium dahliae VdAve1 (Verticillium dahliae Avirulence on Ve1 tomato) effector. In V. dahliae, this effector is exclusively found in race 1 strains and has been shown to be an avirulence protein as recognition by the tomato $V e 1$ receptor leads to resistance against $V$. dahliae strains harboring VdAve1. Besides in C. beticola (CbAve1), homologs of VdAve1 are found in some plant pathogenic fungi such as Fusarium oxysporum (FoAve1) and Colletotrichum higginsianum (ChAve1) as well as in many plant species. Since VdAve1 has been reported to be a virulence factor for $V$. dahliae, Chapter 3 investigates whether the homologs that have been found in C. beticola, F. oxysporum, and Co. higginsianum also contribute to virulence of their producers. While CbAve1 and FoAve1 are shown to be expressed during the fungal infection process and contribute to virulence of their producer, site-directed deletion of ChAve1 in Co. higginsianum had no effect on fungal virulence and no expression was detected during Co. higginsianum on Arabidopsis. Interestingly, earlier studies demonstrated that none of the fungal homologs of VdAve1 were able to complement $\triangle V d A v e 1 V$. dahliae mutants to full virulence. Thus, it is hypothesized that VaAve1 functions differently from the other fungal homologs.

As little is known about the proteinaceous effector repertoire of $C$. beticola, we aimed to identify novel effector proteins. Since C. beticola is a hemibiotrophic fungus, it relies on host cell death induction during the necrotrophic stage of its lifecycle. Chapter 4 describes a phenotype-based approach to identify novel effector proteins that can evoke host cell death. Growing $C$. beticola in Fries medium for seven days resulted in fungal culture filtrate that led to necrosis formation in sugar beet leaves upon infiltration. Mass spectrometry analysis of necrosis-inducing culture filtrate fractions yielded three effector candidates. While two candidates were excluded from further studies due to the lack of effector characteristics or the absence of a necrosis-inducing function, the effector candidate CbNip1 fulfilled all criteria and was further analyzed. The novel necrosis-inducing protein CbNip1 is a small, secreted, cysteine-rich effector protein that is expressed during infection and contributes to C. beticola virulence by inducing necrosis in plant leaves in the absence of light. 
C. beticola is well-known to utilize the perylenequinone cercosporin for necrosis induction during infection. Decades of research on the cercosporin toxin biosynthetic (CTB) pathway identified eight genes that contribute to toxin production. In Chapter 5, phylogenomic analysis revealed that the CTB cluster is larger than previously reported and includes five additional genes located at the $3^{\prime}$ end of the original CTB gene cluster in C. beticola that are essential for toxin production. Among those five genes, two were identified that are responsible for the formation of the methylenedioxy bridge; a unique feature of the cercosporin molecule that is not found in other perylenequinones characterized to date. Furthermore, phylogenomic analysis unveiled that the CTB cluster underwent duplication and horizontal transfer events and is therefore present in a diverse range of plant pathogenic fungi, of which Colletotrichum fioriniae was shown to have the ability to produce cercosporin. Furthermore, gene expression analysis of the Co. fioriniae CTB cluster PKS gene CofCTB1 confirmed that cercosporin production is activated during infection.

The cercosporin molecule displays high structural similarity to other perylenequinone family members such as elsinochrome and phleichrome synthesized by fungi Elsinoë fawcettii and Cladosporium phlei, respectively. Therefore it was hypothesized in Chapter 6 that the biosynthetic gene clusters responsible for production of these perylenequinones are likely to exhibit significant similarity. Based on gene cluster conservation, it was revealed that earlier efforts to identify elsinochrome and phleichrome pathways resulted in misidentification and the biosynthetic pathways claimed to be responsible for their production are actually involved in DHN-melanin biosynthesis. Furthermore, due to the overall gene conservation within perylenequinone biosynthetic clusters, it was possible to identify the true perylenequinone pathways for elsinochrome in E. fawcettti and phleichrome in C. phlei using the established CTB cluster as reference.

Aiming to shed further light on the effector repertoire of $C$. beticola, this thesis investigated proteinaceous and secondary metabolite effectors of this fungus. Chapter 7 provides an overview of currently known C. beticola effectors and associated mechanisms. 


\section{Acknowledgements}

A clear advantage of doing a "sandwich" PhD is that you get to make friends at two different locations. There have been so many wonderful people who have contributed to this thesis and who have made my life so much more colorful and fun. Thank you all for your help and for making this journey so special for me!

First of all, I feel incredibly fortunate to have the two coolest people in the world as promoters. Without Bart and Melvin, this thesis would simply not have been possible. Thank you both for creating a positive and enthusiastic working environment, it made occasional set-backs much easier to deal with. Watching you guys has also shown me that it is perfectly possible to be honest and kind and still be successful in science. I also really appreciate that you not only guided me through the PhD but you also prepared me for the next step and I am now looking forward to finding my place in science.

Bart, thanks that I could come to you for any kind of advice. You always had my back and fully supported me in everything I did, while giving me the freedom to develop my own interests and grow as a scientist but also as a person. I admire you for always leading by example and staying true to yourself and your values and I hope that your calmness and your ability to objectively assess situations rubbed a bit off on me. Thanks for everything!

Melvin, I know it is getting old because I have been saying this for the last 4 1/2 years but I am still convinced that coming to Fargo was without a doubt the BEST decision I have ever made in my life! From day one you had complete trust in my skills and fully supported me in what I did and I cannot thank you enough for that! You are not only my promotor but you became a really good friend and I know that I can rely on you 100\%. Thanks! And an especially big 'Thank you!' also goes to your family. Starting with Tonya, Ava and Wren for always being awesome, helpful and fun to hang out with and also to your parents and your siblings and their families, and all the other Boltons and Beckers who I got to meet, for making me feel welcome at all times. You and your whole family were all so kind to me and made my stay in Fargo incredibly special! Thanks!!

In theory, this is the part where I was planning on transitioning to thanking everyone chronologically starting with my friends and colleagues from Fargo and followed by the ones from Wageningen but there is one person who stands out and who I can't seem to fit into this scheme. Ronnie, without you this thesis would not have been possible! Thank you so much for answering all my questions with incredible patience, for involving me in projects and also for all the discussions about science and 'real life' that we had.

Since this whole journey started in Fargo, I would now like to thank all my colleagues and friends in the US. First of all: the Bolton lab. Jon N., you have no idea how happy I was when you joined our lab. I very much enjoyed working with you and your positive attitude made trouble-shooting all those assays bearable. Becky, I am so glad we got to meet! I just love how we instantly clicked and I hope you will come and visit the next time you are in Europe. I wish you all the best for your PhD! Kathrin, I would like to thank you for all those nice discussions and especially for bringing 
back loads of gummibears and powdered soup packages from your visits in Germany. Subi, Zoey, John Weiland and Nick thanks for making my time at the Bolton lab so much fun.

Jeff, you have been a mentor to me and I am incredibly thankful that you introduced me to the wonderful world of organic chemistry. I really appreciate that you never grew tired of answering all my questions and went above and beyond to support me on the beticolin project. It was very important to me to have you as a critical discussion partner and I am sure that without these talks I would not have been able to finally identify the beticolin inducing conditions.

Gary, thank you so much for hosting me at NDSU and Viv, for being a source of extensive C. beticola knowledge! Without you guys and the support of NDSU, this thesis would not have turned out so well. I am especially glad that we also got to do fun, non-scientific things together like exploring Amsterdam!

There are so many more people who I would like to thank: Ed, John Eid, Karen, and Larry thank you for all the nice discussions we had. Tim, you were a great external advisor! Thank you for letting me use your lab equipment and that I could always come to you for advice. I really appreciate all the discussions we had about science and everything. Thanks! Lori, thank you for always staying on top of things and for being my go-to-person when I needed a hug. Tabitha (NDSU), you know everything about visa and you are one of the most helpful people I have ever met, thanks! And of course Allen and Lisa and their family for being so kind and incredibly supportive. Allen, I would have loved to meet you in Germany and I am so sorry that it did not work out!! But I'm sure we will meet again sometime in the future. And last but certainly not least: Joe. You are like superman! How can I ever thank you for everything you did (ranging from repairing our lab machines to taking me fishing to LOTW or helping me again and again with the Pacifica). You were always there for me and never grew tired of cheering me up when I needed it or convincing me to take a break and go for a beer with you when I was getting tired. Thanks!!!

My life in Fargo would not have been the same without the people from Red River Judo. Thanks for everything, guys! Playing judo and teaching kids class not only was the best way to get my mind off my experiments, it also helped me to find the best friends I could have asked for. Mike, thank you so much for introducing me to burgertime and five guys!!! I enjoyed teaching kid's class with you and whenever you and Leah are in Europe again, let me know! Sam, you are one crazy, kickass lady and I am lucky to call you my friend. With you it is never boring and hanging out with you means to better be ready for some spontaneous, awesome adventures. I hope we will meet again soon! And of course, Josh. I still remember the first time we met and after chatting for like 10 min you said to me: "You don't know it yet, but we will be best friends by the time you leave." And you were so right!!! I want to thank you for all the fun times we had, for making me laugh, for helping me at any time and for being the best friend possible.

A special thanks goes to Amber. I'm not gonna lie, when I first replied to your roommate ad on craigslist, I was simply hoping for someone who's not a complete whacko... little did I know that I would get the best roommate ever! I wish you, Mike, Peyton and Iggy all the best for the future! 
While I very much enjoyed my time in Fargo, I also loved to be back in Wageningen. First and foremost, I would like to thank all the (former and current) members of the Verticillium group. You guys are like family and I am very fond of every single one of you. Martin, Mireille, Yin, Xiaoqian, Jingling, Gabriel, Nelia, Shuhei, Edgar, Jasper V., David, Eduardo, Jordi and Luigi thanks for all your advice and always being happy to discuss and trouble shoot with me. Hanna, I value our friendship a lot. Thank you for your companionship in the lab and for all the help with getting me settled in Cologne. Jasper D., thanks for your patience and for being so fun and creative; I am looking forward to what is yet to come! Katharina, Schätzelein, vielen lieben Dank für alles!! Ich hoffe du kommst bald mal zu Besuch! Luis, I enjoyed working on the review with you a lot and I hope that we will stay in tough in the future. Und Michael, thanks for checking on me from time to time just make sure that I'm okay and for patiently waiting for me to be ready to share. Talking with you about literally everything was always fun and it sure helped me with making some tricky decisions. Thanks!!

To my paranymphs, Hui and Nick. Hui, you are the sweetest person I know and one of my best friends! I value you for your honesty, reliability, and especially for your happy and positive attitude. You do science with a smile and are always willing to share your expertise and (double digested) vectors. Also thank you for always having a backup of buffers, antibiotics, IPTG, super competent DH5a and advice. And of course for making the most delicious food ever! With you and your "at least one dish per person" policy, our little group never left hungry. Nick, thank you for your everlasting enthusiasm for science. While your thoughts and actions are always well reasoned, you do have a playful side and with you research is fun! No matter what time of the day, you are always up for talking science! Thank you for all the hours of discussions, forming hypotheses, discarding said hypotheses, and formulating new ones again. And for always being willing to share your impressive knowledge on proteins with me!

I would also like to thank my MSc student Roger. You were my first student and you sure set the bar high for every other student to follow. I wish you all the best for your future!

Jan, ever since my MSc you have been supporting me. I could always come to you and ask for your advice. Thanks a billion times! Furthermore, I would like to thank Ali for keeping everything organized and Grardy, Ester, and Laurens for making sure we are stocked up and all set to do our research.

To all my mates from the writing room: Aranka, Jingling, Chara, Fernando, and Nani. If I had to pick a bunch of people to be locked into a tiny room with I would totally pick you guys over and over again! The last stretch of the PhD is certainly the most intense but I loved how our little group managed to always find ways to release pent-up energy from sitting too much or help each other to overcome writing blocks or other problems. While the craziness was kind of strong in this room (and I still think that the room would totally benefit from having rubber walls) I have to say, it was a fun ride!

Maikel, Johann, Shuquin, Kiki, Elisa, Klaas, Javier, Jinbin, Sander, Jingling and all my fellow PhDs, the postdocs and everyone else at Fyto, thanks for all the interesting discussions at the coffee table and in the lab. 
A very big 'Thank you!' goes to Bert and Bertus for taking excellent care of my plants and providing me with loads of left-over Benthas!

Robin, Dirk, and Mark, thank you for your friendship.

Thijs, I appreciate a lot that you invested to so much time and energy into designing this thesis! I think it is perfect!! You not only played a very important role in tweaking this thesis into its final shape but also you and the guys from the reception always made sure that I would get every experiment done before radix closes at night! You guys have no idea how much easier you have made my life with that!!!!! Thanks!!

Ich möchte auch meinen langjährigen Freunden danken, die dank Skype und unermütlichem Fernweh immer für mich da waren. Anke, Josi, Kerstin, Katrin, und Sandra danke für eure Freundschaft.

Zum Schluss möchte ich meinen Eltern danken. Mama und Papa, danke, dass ihr mich bei allem so bedingungslos unterstützt. Ich weiss, dass es nicht immer leicht für euch ist nachzuvollziehen, warum ich mich gerade für diesen Weg entschieden habe und warum ich bereit bin so Manches dafür auf mich zu nehmen. Trotzdem akzeptiert ihr einfach alle meine Lebensentscheidungen und helft mir nach Kräften meine Träume zu verwirklichen. Ich danke euch aus tiefstem Herzen dafür! 
Malaika K. Ebert was born in Frankfurt a. M. (Germany) on May 8th, 1985. In 2007, she started her BSc in Agricultural Science at the University of Bonn and wrote her BSc thesis on 'The effect of Venturia inaequalis on chlorophyll content, photosynthetic efficiency and fluorescence of apple leafs' under the supervision of Dr. Ulrike Steiner.

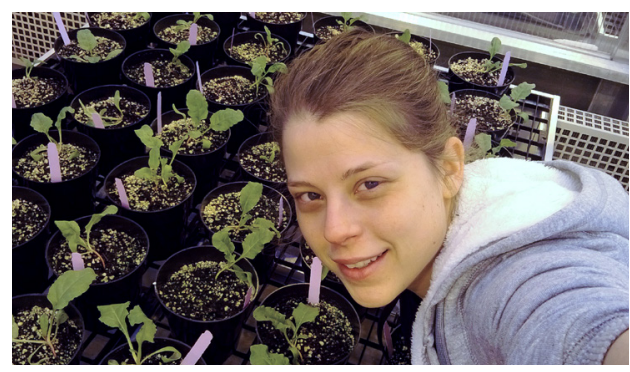

In 2010, Malaika started an MSc in Plant Science with a specialization in Plant Pathology and Entomology at Wageningen University. For her first MSc thesis, she joined the group of Prof. Dr. Bart Thomma at the Laboratory of Phytopathology and, under supervision of Dr. Anja Kombrink, worked on the project titled 'The influence of LysM effectors in fungal virulence and in interactions with other microorganisms.' Since she also wanted to learn about the interaction of plants with beneficial microbes, Malaika joined the group of Dr.ir. René Geurts at the Laboratory of Molecular Biology to perform her second MSc thesis. Under the supervision of Arjan van Zeijl, she identified and characterized ACC synthase genes in Parasponia and Trema.

In 2014, Malaika was appointed as a Sandwich PhD under the joined supervision of Prof. Dr. Bart Thomma (WUR) and Dr. Melvin Bolton (USDA, NDSU) to unravel the effector biology of the sugar beet pathogen Cercospora beticola. After an initial two years at the laboratory of Dr. Melvin Bolton at the U.S. Dept. of Agriculture and North Dakota State University, Malaika returned to the team of Prof. Dr. Bart Thomma for the second half of her PhD. Later that year, Malaika was awarded the EPSO Young Plant Scientist award 2016 in applied research for the suggestion to engineer durable disease resistance by transferring fungal toxin auto-resistance genes to plants. Since July 2018, Malaika is a postdoctoral researcher in the group of Prof. Dr. Gunther Doehlemann at the University of Cologne where she investigates the effector repertoire of the smut fungus Ustilago maydis. 
de Jonge R.*\#, Ebert M.K*, Huitt-Roehl C.R.*, Pal P., Suttle J.C., Neubauer J.D., Jurick W.M., Secor G.A., Thomma B.P.H.J., Van de Peer Y., Townsend C.A.\#, Bolton M.D.\# (2018) Gene cluster conservation provides insight into cercosporin biosynthesis and extends production to the genus Colletotrichum. Proceedings of the National Academy of Sciences. DOl: 10.1073/ pnas. 1712798115

*.\# The contribution of these authors should be considered equal.

Rodriguez-Moreno L.*, Ebert M.K.*, Bolton M.D.\#, Thomma B.P.H.J.\# (2018) Tools of the crook infection strategies of fungal plant pathogens. The Plant Journal. DOl: 10.1111/tpj.13810

*.\# The contribution of these authors should be considered equal.

Bolton MD, Ebert M.K.*, Faino L*, Rivera-Varas V, de Jonge R, van de Peer Y, Thomma BPHJ, Secor G (2016). RNA-Sequencing of Cercospora beticola DMl-sensitive and -resistant isolates after treatment with tetraconazole identifies common and contrasting pathway induction. Fungal Genetics and Biology. DOI: https://doi.org/10.1016/j.fgb.2016.04.003

* The contribution of these authors should be considered equal.

Ebert M.K.*, Spanner R.E.*, de Jonge R.*.\#, Smith D.J., Holthusen J., Secor G.A.,Thomma B.P.H.J., Bolton M.D\#. Gene cluster conservation identifies melanin and perylenequinone biosynthesis pathways in multiple plant pathogenic fungi. Submitted.

*.\# The contribution of these authors should be considered equal.

Ebert M.K., Wang X., Friesen T.L., de Jonge R., Neubauer J.D., Secor G.A., Thomma B.P.H.J., Bolton M.D. Identification and characterization of Cercospora beticola necrosis-inducing effector CbNip1, Submitted.

Boshoven J.C., Ebert M.K.*, Song Y.*, Rovenich H., Rojas Padilla E., Bolton M. D., Thomma B.P.H.J. Homologs of Verticillium dahliae effector Ave1 contribute to virulence of various fungal pathogens. Submitted.

* The contribution of these authors should be considered equal.

Ebert M.K., Thomma B.P.H.J., Bolton M.D. Perylenequinones in plant pathology. In preparation.

De Jonge R., Bian Z., Webb K.M., Ebert M.K., Spanner R.E., Shrestha S., Bolton M.D. Identification and characterization of Fusarium secorum effector proteins. Author order is not fixed. In preparation. 


\title{
Education Statement of the Graduate School Experimental Plant Sciences
}

\author{
Issued to: Malaika Karolina Ebert \\ Date: \\ 6 September 2018 \\ Group: \\ Laboratory of Phytopathology \\ University: Wageningen University \& Research
}

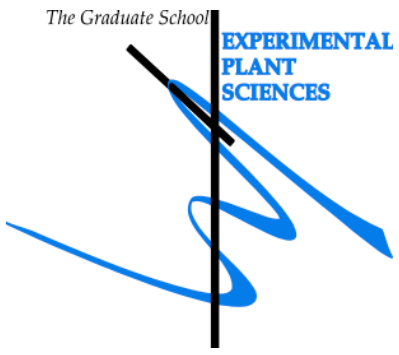

\begin{tabular}{|c|c|c|}
\hline \multicolumn{2}{|c|}{ 1) Start-up phase } & $\underline{\text { date }}$ \\
\hline 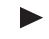 & First presentation of your project & \\
\hline \multirow{3}{*}{ 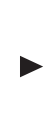 } & Title: Disease management and effector biology of the sugar beet pathogen Cercospora beticola & 02 Feb 2014 \\
\hline & Writing or rewriting a project proposal & \\
\hline & Title: Disease management and effector biology of the sugar beet pathogen Cercospora beticola & 11 Mar 2013 \\
\hline \multirow[t]{2}{*}{ D } & Writing a review or book chapter & \\
\hline & $\begin{array}{l}\text { Tools of the crook - infection strategies of fungal plant pathogens, submitted to The Plant } \\
\text { Journal }\end{array}$ & 15 Nov 2017 \\
\hline \multirow{3}{*}{$>$} & MSc courses & \\
\hline & Laboratory use of isotopes & \\
\hline & Subtotal Start-up Phase & 13.5 credits* \\
\hline
\end{tabular}

\begin{tabular}{|c|c|c|}
\hline \multicolumn{2}{|c|}{ 2) Scientific Exposure } & \multirow[t]{2}{*}{$\underline{\text { date }}$} \\
\hline \multirow{5}{*}{$>$} & EPS PhD student days & \\
\hline & EPS PhD Student days 'Get2Gether', Soest, NL & 09-10 Feb 2017 \\
\hline & EPS PhD Student days 'Get2Gether', Soest, NL & 15-16 Feb 2018 \\
\hline & EPS theme symposia & \\
\hline & $\begin{array}{l}\text { EPS Theme } 2 \text { symposium 'Interactions between Plants and Biotic Agents', together with } \\
\text { Willie Commelin Scholten day, Leiden, NL }\end{array}$ & 22 Jan 2016 \\
\hline \multirow{6}{*}{$>$} & $\begin{array}{l}\text { EPS Theme } 2 \text { symposium ' Interactions between Plants and Biotic Agents', together with } \\
\text { Willie Commelin Scholten day, Wageningen, NL }\end{array}$ & 23 Jan 2017 \\
\hline & EPS Theme 3 symposium “Metabolism and Adaptation”, Wageningen, NL & 14 Mar 2017 \\
\hline & National meetings (e.g. Lunteren days) and other national platforms & \\
\hline & Annual meeting 'Experimental Plant Sciences', Lunteren, NL & 11-12 Apr 2016 \\
\hline & Annual meeting 'Experimental Plant Sciences', Lunteren, NL & 10-11 Apr 2017 \\
\hline & Seminars (series), workshops and symposia & \\
\hline \multirow{3}{*}{$>$} & Seminars: & \\
\hline & Prof.dr. Jane Parker, Resistance pathway dynamics in plant immunity, Wageningen & 21 Jan 2016 \\
\hline & $\begin{array}{l}\text { Prof. Laura Grenville-Briggs 'Molecular Oomycete-Host Interactions: The Good, the Bad } \\
\text { and the ugly', Wageningen }\end{array}$ & 19 Feb 2016 \\
\hline
\end{tabular}




\begin{tabular}{|c|c|c|}
\hline & $\begin{array}{l}\text { Jan Ruijter "Analysis of qPCR data. The use and usefulness of amplification curve analy- } \\
\text { sis", Wageningen }\end{array}$ & 14 Mar 2016 \\
\hline & Dr. Olivier Hamant 'How do plants read their own shape ?', Wageningen & 16 Mar 2016 \\
\hline & $\begin{array}{l}\text { Prof. Alain Tissier 'Insights into the inner workings of a metabolic cell factory: the tomato } \\
\text { glandular trichome' }\end{array}$ & 18 Mar 2016 \\
\hline & Prof.dr. Douglas Mitchell 'Genomics-enabled natural products discovery', Wageningen & 31 Mar 2016 \\
\hline & Prof. Caitilyn Allen, 'How Ralstonia solanacearum succeeds in plant xylem vessels', Wageningen & 29 Apr 2016 \\
\hline & Workshops: & \\
\hline & COST Israel,Tel Aviv, Israel & 10-12 Feb 2015 \\
\hline & Metabolomics in Chemical Ecology, NIOO, Wageningen, NL & $\begin{array}{l}31 \text { Oct-01 Nov } \\
2016\end{array}$ \\
\hline & COST annual meeting, Bled, Slovenia & 01-03 Mar 2017 \\
\hline & Symposia: & \\
\hline & $\begin{array}{l}\text { 3rd Wageningen PhD Symposium "Diversity of Science" - entitled Learn from your enemy } \\
\text { - transferring a pathogen-derived toxin resistance mechanism to crop plants for durable } \\
\text { disease resistance }\end{array}$ & 26 Apr 2016 \\
\hline 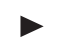 & Seminar plus & \\
\hline$>$ & International symposia and congresses & \\
\hline & APS annual meeting Minneapolis, MN, USA & 09-13 Aug 2014 \\
\hline & Asilomar Fungal Genetics, Pacific Grove, CA, USA & 17-22 Mar 2015 \\
\hline & $\begin{array}{l}\text { ASSBT - American Society of Sugar Beet Technologists biennial meeting, Clearwater } \\
\text { Beach, FL, USA }\end{array}$ & 23-26 Feb 2015 \\
\hline & EPSO Plant Biology Europe, Prague, CZE & 26-30 Jun 2016 \\
\hline$>$ & Presentations & \\
\hline & Talks: & \\
\hline & APS “Characterization of novel Cercospora beticola effector proteins “ Minneapolis, USA & 11 Aug 2014 \\
\hline & $\begin{array}{l}\text { ASSBT “The Cercospora beticola effector CbAve1 promotes virulence during sugar beet } \\
\text { infection”, Clearwater Beach, USA }\end{array}$ & 24 Feb 2015 \\
\hline & $\begin{array}{l}\text { COST "Identification and characterization of a novel Cercospora beticola effector protein", } \\
\text { Tel Aviv, Israel }\end{array}$ & 12 Feb 2015 \\
\hline & $\begin{array}{l}\text { Lunteren "Identification of fungal toxin auto-resistance genes and their potential to provide } \\
\text { durable resistance in crop plants", Lunteren, NL }\end{array}$ & 11-12 Apr 2016 \\
\hline & $\begin{array}{l}\text { 3rd Wageningen PhD Symposium "Diversity of Science" - entitled Learn from your enemy - } \\
\text { transferring a pathogen-derived toxin resistance mechanism to crop }\end{array}$ & 26 Apr 2016 \\
\hline & $\begin{array}{l}\text { Molecular Genetics meeting "Fungal toxin self-resistance genes and their potential to } \\
\text { provide durable resistance in crops", Wageningen, NL }\end{array}$ & 21 Oct 2016 \\
\hline & $\begin{array}{l}\text { COST Annual meeting "Transfer of fungal-derived toxin tolerance to crop plants to engi- } \\
\text { neer resistance to Cercospora diseases" Bled, Slovenia }\end{array}$ & 01-03 Mar 2017 \\
\hline & Poster: & \\
\hline & $\begin{array}{l}\text { Fungal genetics conference "Characterization of the novel Cercospora beticola necro- } \\
\text { sis-inducing effector CbNIP10", Pacific Grove, USA }\end{array}$ & 17-22 Mar 2015 \\
\hline$>$ & IAB interview & \\
\hline 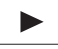 & Excursions & \\
\hline & Subtotal Scientific Exposure & 20.7 credits* \\
\hline
\end{tabular}




\begin{tabular}{|c|c|c|}
\hline \multicolumn{2}{|c|}{ 3) In-Depth Studies } & $\underline{\text { date }}$ \\
\hline \multirow[t]{3}{*}{ 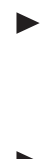 } & EPS courses or other PhD courses & \\
\hline & Your Plant Science NIAB PhD Winter School, UK & 20-21 Nov 2017 \\
\hline & Advanced course 'Data analysis and visualizations in R (for biologists)', Wageningen, NL & 12-13 Dec 2016 \\
\hline \multirow{3}{*}{$>$} & Journal club & \\
\hline & Individual research training & \\
\hline & $\begin{array}{l}\text { FARGO, Dr Melvin Bolton, sugar beet unit, United States Department of Agriculture, Fargo, } \\
\text { USA }\end{array}$ & $\begin{array}{l}02 \text { Feb 2014-31 } \\
\text { Dec } 2015\end{array}$ \\
\hline \multicolumn{2}{|r|}{$\begin{array}{ll} & \text { Subtotal In-Depth Studies }\end{array}$} & 4.2 credits* \\
\hline
\end{tabular}

\begin{tabular}{|l|l|c|}
\hline 4) Personal development & date \\
\hline \multicolumn{1}{|l}{} & Skill training courses & 11 Feb 2017 \\
\cline { 2 - 3 } & EPS Introduction Course, Wageningen, NL & 17 Mar 2016 \\
\cline { 2 - 3 } & Reviewing a scientific paper, Wageningen, NL & 09 Sep-22 Nov \\
\cline { 2 - 3 } & Writing grant proposals, Wageningen, NL & 2016 \\
\hline & Organisation of PhD students day, course or conference & $09-10$ Feb 2017 \\
\hline Get2Gether - pubquiz & 2016 and half \\
\cline { 2 - 3 } & Flying seminars (Mak Varrelmann, 18 Apr 2017; Urs Wyss, 2 Oct 2017) 2017 \\
\hline & Membership of Board, Committee or PhD council & 2016 and half \\
\hline
\end{tabular}

Herewith the Graduate School declares that the $\mathrm{PhD}$ candidate has complied with the educational requirements set by the Educational Committee of EPS which comprises of a minimum total of 30 ECTS credits

${ }^{*}$ A credit represents a normative study load of 28 hours of study. 
This work was supported by the Netherlands Organization of Scientific Research (NWO) grant 833.13.007, USDA - ARS CRIS project 3060-21000-044-D and grants from the Beet Sugar Development Foundation and the Sugar Beet Research and Education Board of MN and ND. The work was performed in the Sugarbeet and Potato Research Unit, USDA- ARS Fargo, ND, USA and in the Laboratory of Phytopathology, Wageningen University, The Netherlands.

Cover \& Layout design: Thijs Dueck

Print: proefschriftmaken.nl 
